\title{
PSYCHOLOGICAL PREPARATION FOR PATIENTS UNDERGOING DAY SURGERY
}

\section{Mark MITCHELL}

\author{
School of Nursing \\ University of Salford, Greater Manchester, \\ United Kingdom.
}

Submitted in Partial Fulfilment of the Requirements of the Degree of Doctor of Philosophy,

April, 2002 


\section{CONTENTS}

Contents

Tables

Diagrams

Figures

Appendices

Chapter 1 Introduction

Ethos

Day Surgery

Introduction

Psycho-dynamic Approach to Coping

Transactional Approach to Coping

Convergent or Combined Approach to Coping 25

Singular Approaches

Personality -

Neuroticism

Vigilant and Avoidant Behaviour

Social Cognitions -

Health Locus of Control

Self-efficacy

Social Support

Optimism

Convergent or Combined Psychological

Focus for Day Surgery

Restrictions to Current Approaches

Future Direction 


\section{Chapter 3}

Information Provision Prior to Surgery

Information Categories

Problem Focused Coping Information -

Procedural or Situational Information

Behavioural or Role Information

Sensory Information

Emotional Focused Coping Information -

Cognitive or Emotional Coping Strategies

Relaxation Information

Modelling Information

Information Delivery

Literature Review

Nursing and Anaesthetic Practice 100

Information Provision

108

Patients' Experience in the Day Surgery Unit 
Information Provision

Patient Anxiety

Adjustment to Surgery

Health Locus of Control

Self-Efficacy

Future Research

256

Study Limitations

258

Chapter 7

Recommended Nursing Intervention

Psycho-educational Preparation for Day

Surgery

269

Implementation into Day Surgery

273

Summary of Recommendations

282

Appendices

285

References

Bibliography 


\section{TABLES}

NUMBER

$1 \quad$ Psycho-educational information categories. 62

2 Classification of all studies reviewed concerning patients' perceptions of day surgery.

3 Summary of the studies into patients' perceptions of day surgery - nursing and anaesthetic practice.

4 Summary of the studies into patients' perceptions of day surgery - information provision.

5 Summary of the studies into patients' perceptions of day surgery - patients experiences in the day surgery unit.

6 Summary of the studies into patients' perceptions of day surgery - recovery at home.

7 Operational definitions.

8 Overview of the information booklets sent via post 7 - 10 days prior to surgery.

9 Behavioural adjustment rating questionnaire.

10 Participant satisfaction rating questionnaire.

$112 \times 2$ unrelated factorial design for vigilant and avoidant coping style.

12 Information sent via post 7 - 10 days prior to surgery.

13 Overview of the quasi-experimental research design.

14 Research interview schedule.

15 Abbreviated independent variables (utilised for plotting and analysis). 
16 Abbreviated dependent variables from all questionnaires (utilised for analysis).

17 Laparoscopic gynaecological surgery undertaken.

18 Specific surgery undertaken.

19 Coping styles possessed by participants combined with information booklet received.

20 Descriptive statistics for vigilant coping behaviour (all participants).

21 Descriptive statistics for avoidant coping behaviour (all participants).

22 Distribution of information booklets to vigilant and avoidant copers

23 Descriptive statistics for vigilant coping behaviour (vigilant copers only).

24 Descriptive statistics for avoidant coping behaviour (avoidant copers only).

25 Satisfaction with information booklet.

26 Overall satisfaction with information.

27 Requested amount of information.

28 Nurses' ratings of information required. 201

29 Self-rated state anxiety. 203

$30 \quad$ Nurse-rated anxiety. 205

31 Adjustment to surgery. 209

32 Internal health locus of control. 211

33 Chance health locus of control. 212 
NUMBER

34 External health locus of control (powerful others). 214

35 Self-efficacy. 217

36 Psycho-educational management plan. 273

37 Standard 7 - Clinical negligence scheme for trusts. 278

$38 \quad$ Proposed information pathway chart. 279 


\section{DIAGRAMS}

NUMBER

$1 \quad$ Psycho-dynamic coping process. 20

$2 \quad$ Transactional coping process. 24a

$3 \quad$ Convergent or combined coping process. 26

$4 \quad$ Intra-psychic coping strategies. 32

$5 \quad$ Coping with day surgery - convergent or combined approach. $57 a$

$6 \quad$ Frequency of vigilant and avoidant coping

(all participants). 186 


\section{FIGURES}

NUMBER

$1 \quad$ Research study country of origin. 98

2 Plot of possible theoretical relationship between independent variables (information provided $X$ coping style) on dependant variable (satisfaction with information booklet).

3 Plot of possible theoretical relationship between independent variables (information provided $X$ coping style) on dependant variable (satisfaction with information).

4 Plot of possible theoretical relationship between independent variables (information provided $X$ coping style) on dependant variable (information requested).

5 Plot of possible theoretical relationship between independent variables (information provided $X$ coping style) on dependant variable (behavioural information).

6 Plot of possible theoretical relationship between independent variables (information provided X coping style) on dependant variable (self-rated state anxiety).

$7 \quad$ Plot of possible theoretical relationship between independent variables (information provided $X$ coping style) on dependant variable (behavioural anxiety).

8 Plot of possible theoretical relationship between independent variables (information provided $X$ coping style) on dependant variable (observed adjustment to surgery).

9 Plot of possible theoretical relationship between independent variables (information provided X coping style) on dependant variable (internal health locus of control). 
10 Plot of possible theoretical relationship between independent variables (information provided $X$ coping style) on dependant variable (chance health locus of control).

11 Plot of possible theoretical relationship between independent variables (information provided $X$ coping style) on dependant variable (external health locus of control).

12 Plot of possible theoretical relationship between independent variables (information provided $X$ coping style) on dependant variable (self-efficacy).

13 Vigilant coping behaviour distribution (all participants).

14 Avoidant coping behaviour distribution (all participants).

15 Vigilant and avoidant coping plot.

16 Vigilant coping behaviour distribution (vigilant copers only).

17 Avoidant coping behaviour distribution (avoidant copers only).

18 Non-significant main effect (coping style $X$ anxiety).

19 Significant main effect (coping style $X$ nurse-rated anxiety).

20 Non-significant interaction effect (coping style $X$ nurse-rated anxiety).

21 Non-significant main effect (coping style $X$ chance health locus of control).

22 Significant main effect (coping style $X$ external health locus of control).

23 Significant main effect (coping style $X$ self-efficacy). 
24 Non-significant interaction effect (coping style $X$ self-efficacy). 


\section{APPENDICES}

NUMBER

1 Basket of Procedures (Audit Commission 1990) and the British Association of Day Surgery 'Trolley of Procedures' (Cahill 1999).

2 Ethical committee letter of approval.

3 Extended information booklet.

$4 \quad$ Simple information booklet.

$5 \quad$ Covering letter following initial contact.

6 Consent form.

$7 \quad$ Mainz Coping Inventory (Krohne 1989).

8 State-trait anxiety inventory (Spielberger et al 1983) in shortened format for State anxiety only (Marteau and Bekker 1992).

9 Multi-dimensional Health Locus of Control scale (Form A) (Wallston et al 1978).

10 Self-efficacy questionnaire (Jerusalem and Schwarzer 1992).

11 Behavioural Adjustment Rating questionnaire.

12 Participant Satisfaction Rating questionnaire.

13 Original pre-operative questionnaire booklet. 


\section{ACKNOWLEDGEMENTS}

A sincere thank you to Dr. Peter Eachus for his sustained advice and guidance throughout this study. To Mrs. Anne Mottram for her enthusiasm for day surgery and extensive, continued help. Considerable gratitude also belongs to my family for their constant support and encouragement. Especially to my father who recognised I could undertake such work but is sadly not here to see it. To the medical, nursing and clerical staff at Hope Hospital Day Surgery Unit, Greater Manchester for their invaluable time and effort. Furthermore, I am indebted to Professor H. W. Krohne from the University of Mainz, Germany for allowing the use of his leading questionnaire on vigilant and avoidant coping behaviours. Finally, I am very grateful to all the participants for their kind help and cooperation at such an anxious time. Without the contribution of all these people this study would never have been possible. 


\begin{abstract}
The aim of the study was to discover the most suitable methods of psychologically preparing patients for modern day surgery and to formulate a pre-operative nursing plan which embraces such methods. Contemporary evidence suggests the level of information provided, individual coping style, anxiety and social cognitions may strongly influence the psychological status of patients undergoing surgery. However, the most crucial aspect within day surgery may concern the degree to which the level of information provided meets with individual requirements i.e. information matched with maximum (vigilant coper) and minimum (avoidant coper) levels of requirement. A
\end{abstract} convenience sample of 120 patients undergoing intermediate, non-life threatening, gynaecological laparoscopic day surgery were contacted prior to admission and randomly assigned into two groups. Group I received an extended information booklet and Group II a simple information booklet. All participants received a questionnaire pack pre-operatively which was utilised to determine coping style, anxiety, health locus of control and self-efficacy. Initially, the nurses rated participants in receipt of the extended information as less anxious, irrespective of coping style $(F(1,47)=4.257, p=0.45)$. However, irrespective of the information booklet received, participants with a vigilant coping style encountered greater external health locus of control $(F(1,47)=$ 4.249, $p=0.045)$. Again, irrespective of the information booklet received, participants with a vigilant coping style also experienced lower self-efficacy ( $F$ $(1,47)=6.173, p=0.017)$. As participants in receipt of the extended 
information booklet were observed to be less anxious, the ability of the booklet to alleviate anxiety is discussed. Additionally, regardless of information received, vigilant coping behaviour was associated with diminished health locus of control and lower self-efficacy. Such sub-optimal appraisals are equally explored. An innovative plan is proposed providing explicit psycho-educational guidance for intervention throughout modern elective day surgery. Incorporation of this strategy into nursing practice is outlined and techniques for implementation recommended. 


\section{ChAPTER 1 \\ Introduction}

\section{Ethos}

Advances in medical practice together with increasing healthcare costs have resulted in a decline in the number of days patients spend in hospital (Audit Commission 1990). During the past 25 years the length of hospital stay for a patient in the United Kingdom has fallen by almost $50 \%$ and this trend is set to continue. The extensive volume and complexity of day surgery activity provides one clear example of this healthcare transformation. Wide-ranging advances in surgical and anaesthetic practices have enabled innovative, expansive progress for day surgery. Such medical advances have pioneered modern day surgery development and given rise to its considerable success. However, medical advances in surgical and anaesthetic techniques have tended to dominate the day surgery agenda, to the exclusion of many other aspects of care. One such aspect concerns the psycho-educational management of patients. Psychoeducational intervention is defined here as the purposeful attempt to provide tangible aspects of care aimed at enhancing an individual's psychological status together with the planned provision of educational material.

Such psychological considerations may have been inadvertently overlooked in recent years in favour of the above crucial medical issues. However, effective pre-operative psycho-educational management was rarely considered on a formal basis prior to the expansion of day surgery (Cahill 1998, Diamond 1995, 
Mitchell 1997). As little nurse/ patient contact now occurs prior to admission and considerably less contact occurs on the day of surgery, the need for improved psycho-educational intervention in modern day surgery is imperative. The aims of this study are therefore twofold. To discover the most suitable methods of psychologically preparing patients for modern day surgery and to formulate a pre-operative nursing plan which embraces such methods.

Finally, the field of psychology can be very briefly described as the 'study of individual human behaviour'. Therefore to make 'general' recommendations regarding the psychological care of individual patients may be a contradiction in terms, as applied psychological research cannot provide absolute answers to all individual questions. Nevertheless, answers gained from the applied psychological research associated with day surgery, may provide an effective guide for nursing intervention in a rapidly changing healthcare environment. If the psychological principles gained from this research study can be integrated into day surgery nursing practice, the fear and apprehension experienced by many patients prior to day surgery may be more effectively managed. Providing tangible aspects of care aimed at enhancing the patient's psychological status and the planned provision of educational material to support such interventions is, in the author's opinion, a fundamental part of every surgical nurse's role and therefore the foundation upon which this study rests. 


\section{Day Surgery}

Day surgery represents a major shift in the delivery of surgical healthcare in the United Kingdom. Intermediate elective surgical episodes once requiring lengthy hospital admission have disappeared, never to return (intermediate elective surgery is defined here as planned uncomplicated surgery under general anaesthesia which can be undertaken in an operating theatre in less than one hour). This has had, and is increasingly having, a major impact on the delivery of the psycho-educational elements of care as the length of time patients spend in hospital has been dramatically cut. For example, patients are commonly admitted 1 to 2 hours prior to day surgery with minimal time for nurse/ patient interaction. Following surgery, patients are normally dressed and ready to go home, again within 1 to 2 hours. The average length of stay in a day surgery unit within Europe is therefore currently 61/2 hours (Pfisterer et al 2001). Such persistent time restrictions habitually ensure medical aspects of care take preference over many other interventions i.e. psycho-educational elements of care. The pioneering growth and subsequent expansion in day surgery healthcare within the United Kingdom will therefore be briefly discussed below. The delivery of psycho-educational intervention will then be outlined together with its historical roots.

\section{Growth and Expansion}

Day surgery growth has developed as a result of medical advancement and economic expansion. Growth in this country, although slow in the early 1980's, continues to rise i.e. $46.4 \%(1994 / 5)$ to $60 \%(1997 / 8)$ of all elective surgery (De 
Lathouwer and Poullier 1998). Day surgery is defined in the United Kingdom as "a patient who is admitted for investigation or operation on a planned nonresident basis and who nonetheless requires facilities for recovery." (Royal College of Surgeons 1992 p. 3). In Europe and the United States of America differing definitions and healthcare practices have led to differing rates of day surgery growth. In the United States of America, for example, day surgery or ambulatory surgery is defined as a stay of less than 23 hours whereas in the United Kingdom this would be considered an overnight stay. However, on an international basis, considerably more elective surgery now takes place in day surgery facilities than in the traditional in-patient theatres. Moreover, many countries currently undertaking little day case surgery are eager to promote an increase (Lau et al 2000, McCallum et al 2000).

In addition to the increasing amount of day surgery, the complexity of surgical procedures able to be performed within day surgery facilities also continues to expand (Birch 1994, Jarrett 1997, Cahill 1999, Garcia-Urena et al 2000, Huang et al 2000, Ong et al 2000, Perez et al 2000). The majority of patients treated in day case facilities have welcomed this new form of surgical healthcare as crucially it involves minimal disruption to lifestyle (Garraway et al 1978, Llewellyn 1991, Mudie 1992, Greenwood 1993). Day surgery therefore vividly demonstrates the future for elective intermediate surgery, as vast amounts of traditional in-patient operations will continue to be transferred into day-case procedures. This rapid growth in day surgery has arisen mainly as a result of 
medical advances and the desire for greater cost effectiveness (Audit Commission 1990, 1992, Murphy 1994, Jarrett 1995, 1997).

Firstly, medical advances or more accurately, surgical and pharmacological advances have helped to achieve the expansion of day surgery in differing ways. The operating time or length of time required to perform certain surgical procedures has been greatly reduced. So called 'keyhole surgery' or 'Minimal Access Surgery' is the reason for the considerable reduction in time. As a result of these new surgical techniques the theatre time once required to undertake operations necessitating large surgical incisions and the corresponding length of hospitalisation needed for recovery from such surgical assaults has been dramatically reduced (Hodge 1994, Jarrett 1995). Indeed, Montori (1998) states, "It is no exaggeration to say that minimally invasive surgery has opened up a new form of modern surgery." (p. 244). This has been further enhanced by the use of more rapid acting anaesthetic agents e.g. Diprivan (Propofol) (Ratcliffe et al 1994). Not only can larger surgical procedures now be undertaken in less time but also improved anaesthetic agents allow the patient to become fully conscious in a much shorter period. As a direct result of these advances the Royal College of Surgeons (1992) stated "... day surgery is now considered to be the best option for $50 \%$ of all patients undergoing elective surgical procedures, though the proportion will vary between specialities." (p. 2). In a later study by the Royal College of Surgeons of England and East Anglia R.H.A. (1995) it was revealed all day surgery units surveyed expected a steady increase in their workload. This is indeed proving to be the case as the original 
list of 'Basket Procedures' (list of 20 intermediate surgical procedures deemed suitable for day case surgery) put forward by the Audit Commission (1990) has grown and is now referred to as the 'Trolley of Procedures' (Cahill 1999) (list of approximately 25 intermediate surgical procedures deemed suitable for day case surgery) (Appendix 1).

Secondly, day surgery has the potential to be more cost effective than inpatient surgery i.e. more patients treated for the same amount of money. With the growth of consumerism supported by more formal mechanisms such as the Department of Health's 'Patient's Charter' (1991), a central Government initiative was launched to encourage all N.H.S. Trusts to expand their day surgery facilities (N.H.S. Management Executive Value for Money Unit 1991). The aim was to help decrease the time people spent waiting for operations, improve N.H.S. efficiency and reduce overall running costs (Jarrett 1995). Many N.H.S. Trusts responded and the level of day surgery activity increased overall by $30 \%$ (N.H.S. Management Executive 1993). The report goes on to state $50 \%$ of all elective surgery should be undertaken on a day case basis by $1997 / 8$ with some surgical specialities being able to achieve $80 \%$ by 2000 . In the United States of America, insurance companies aided the expansion of day surgery by refusing to pay for certain in-patient procedures. Although there are many differences between healthcare financing in the United Kingdom and the United States of America, no Government can ignore the cost savings associated with day surgery (Cooper 1997). Indeed, the most recent report by the Audit Commission (2001) regarding day surgery states that if all Hospital Trusts could 
achieve the level of surgery undertaken by the best performing day surgery units, a further 120,000 in-patients per year could be treated on a day case basis.

\section{Psycho-educational Management}

The steady rise in day surgery has, however, presented a considerable challenge to its future effectiveness. Day case patient preparation is dominated by medical fitness for surgery (Markanday and Platzer 1994, Murphy 1994, Dunn 1998, Fellowes et al 1999). In the pursuit of safe, efficient, day-case surgery which ensures a constant throughput of patients, psycho-educational aspects of care have become considerably marginalized (Salvage 1990, Radcliffe 1993, Spitzer 1998, Kleinbeck 2000, Leinonen et al 2001). Whilst ensuring medical fitness for surgery is a vitally important activity, such duties could be widely viewed as medically orientated tasks to ensure surgical safety and the progressive throughput of patients in the limited time available (Reid 1997, Cahill 1998). Such work practices may have embraced challenges geared to maximise day surgery efficiency but inadvertently relegated other crucial patient centred issues i.e. psycho-educational care. Moreover, if such an emphasis is reflective of 'normal' day surgery practice i.e. ensuring medical fitness for, and medical recovery from surgery, it becomes all too apparent why information dissemination has been identified as a considerable challenge for day surgery (Chapter 4).

The delivery of effective psycho-educational care is further compounded in the United Kingdom, as seldom has such intervention been undertaken in the 
way defined by this study i.e. the purposeful attempt to provide tangible aspects of care aimed at enhancing an individual's psychological status together with the planned provision of educational material. Current pre-operative psychological preparation for surgery almost exclusively consists of the provision of a mixture of problem focused and emotional focused coping information only i.e. procedural, behavioural and sensory information, cognitive coping strategies, relaxation and modelling information (Hayward 1975, Boore 1978) (Chapter 3). Additionally, such information is frequently delivered in an unstructured and ad-hoc manner.

The basis for such sub-optimal psycho-educational intervention spans many decades. For a number of years it has been known that admission to hospital, especially for surgical intervention, can cause considerable apprehension (Shipley et al 1978, Pickett and Clum 1982, Ridgeway and Mathews 1982). Studies spanning four decades have identified the causes of such anxiety i.e. the anaesthetic, pain and discomfort, unconsciousness and the operation itself (Egbert et al 1964, Ramsay 1972, Male 1981, McCleane \& Cooper 1990, Mitchell 1997). Several more recent studies have further highlighted the increase in anxiety experienced by patients admitted for day surgery (Mackenzie 1989, Swindale 1989, Caldwell 1991, Markland and Hardy 1993). Despite such evidence, pre-operative psycho-educational management is an aspect of nursing care, which as yet, has no formalised strategy i.e. no identified plan adhered to and replicated by all members of staff. 
Salvage (1990) and Radcliffe (1993) reported psychological nursing care to be seldom undertaken by nurses in a systematic and documented manner. Spitzer (1998) states "Providing quality nursing care means actualising all four aspects of care (physical, emotional, social and managerial). Often, however, under the pressure of a work overload and/ or a high turnover of patients, the physical components of care tend to take over." (p. 790). Kleinbeck (2000), in an American analysis of peri-operative nursing practices also demonstrated the strong prevalence for the physical components of care. Four aspects of perioperative nursing practice were identified i) patient and family behavioural responses to surgery, ii) peri-operative patient safety, iii) peri-operative physiological responses to surgery, and, iv) the health systems required to deliver peri-operative care. It could be argued the psycho-educational element of care appears in the first aspect i.e. patient and family behavioural responses to surgery, although it clearly states 'behavioural responses' suggesting the gaining of a compliant patient is the overall aim. More recently, Leinonen et al (2001) surveyed 874 surgical patients regarding satisfaction with care. Physical nursing activities were deemed excellent while the educational aspects of the nurses' role received many negative comments.

Many studies have recommended pre-operative programmes should not merely involve information provision, as is frequently the case (Wilson-Barnett and Osborne 1983, Stephenson 1988, Swindale 1989, Walsh and Ford 1989, Gould and Wilson-Barnett 1995, Waisel and Truog 1995, Knudsen 1996, Lancaster 1997, Foulger 1997, Ruuth-Setälä, et al 2000). Numerous studies 
have stressed the need for effective programmes of psychosocial support (Badner et al 1990, O'Rourke et al 1991, Mitchell 1994, Thomas 1995, Lindén and Engberg 1995; 1996, Stengrevics et al 1996, De Groot et al 1997b, Heikkilä et al 1998, Shuldham 1999) and many have highlighted the benefits to be gained from such programmes (Devine 1992, Kugler et al 1994b, Garden et al 1996, Klafta and Roizen 1996, Royal College of Surgeons of England and Royal College of Psychiatrists 1997, Salmon and Hall 1997, Lithner and Zilling 1998, Dusseldorp et al 1999, Mott 1999). Moreover, many studies have emphasised the various aspects of psychosocial care and information provision required alongside modern surgical practices (French 1979, Reading 1982, Schoessler 1989, Yount and Schoessler 1991, Lonsdale and Hutchison 1991, Johnston et al 1992, Kratz 1993, Fareed 1994, Gould and Wilson-Barnett 1995, Goodman 1997, Motyka et al 1997, Bradshaw et al 1999, Fellowes et al 1999).

Although frequently recommending improved aspects of care, few studies have provided explicit insight. Hill et al (1992) in a review of the literature only recommends further study stating, "... research that compares the efficacy of two interventions will help us to tease apart the components responsible for change in psychological outcome." (p. 40S). Horne et al (1994) in an overview recommends improved communication and patient control although places the emphasis for change on the medical profession. Heikkilä et al (1998), as with many studies, advocates improved assessment although merely states, "Patient fears could be diminished by developing individualised care." (p. 1234). In a study by Van Zuuren et al (1996) the only firm advice was "...medical staff 
should try to develop a keen eye for the coping styles of their patients." (p. 29). Stengrevics et al (1996) only states "...interventions aimed at improving surgical outcomes by reducing negative psychological states may lead to decreased post-operative complications." (p. 475).

In a recent review of the literature on recovery from surgery, Kiecolt-Glaser et al (1998) put forward a bio-behavioural model. This merely advocates encouraging healthy pre-operative behaviour and effective pain management to help prevent delayed wound repair. Duits et al (1999) argues group therapy and counselling is required in the pre-operative phase by the more neurotic patient but provides no indication as to what this involves or indeed the practical application of such a service. A report by the Royal College of Nursing and Society of Orthopaedic Nursing concerning patient pre-assessment (hospital appointment prior to the day of admission primarily to check medical fitness for surgery and anaesthesia) recommends psychological care should involve assessing coping with the demands of surgery (Fellowes et al 1999). However, the only suggestion put forward is to provide adequate information. A comprehensive study of surgical in-patients by De Bruin et al (2001) also yielded little specific guidance. "The reduction of patients' subjective distress is surely one important goal that justifies the development and employment of these (psychological) programmes." (De Bruin et al 2001 p. 268). However, what constitutes pre-operative psychological programmes of care is not detailed. 
A small number of studies have provided some indication as to the practical care required. In a review of the literature by Salmon (1992b) it is suggested that to focus purely on the patient may be very difficult. Therefore, the focus may have to change to the ward environment as a potential mediator of stress i.e. the level of health control and the provision of wider emotional support. Lonsdale and Hutchison (1991) surveyed 187 patients prior to surgery and discovered meeting their anaesthetist prior to surgery was the most important aspect and having a comprehensive list of the possible complications the least important. Salmon et al (1994) states the main aspect of satisfaction concerning colonoscopy was "... the perception that staff were warm, interested and informative." (p. 345). In a survey of in-patients by Webb and Hope (1995) participants were asked to rank the nursing activities deemed as most important. They rated listening, relieving pain and the instructional aspects of the nurses' role as the most important. Crumlish (1998) interviewed 124 patients before and after general anaesthesia. Participants rated their main pre and post-operative behaviour as "Getting professional help and doing what is recommended." (p. 275). In a study by Reid (1997) 15 day surgery nurses were surveyed and they reported their main role was to empower patients, reduce anxiety and endeavour to meet individual needs.

The Royal College of Surgeons of England and the Royal College of Psychiatrists (1997) put forward a number of broad strategies concerning psychological management i.e. varying degrees of information provision, emotional support, privacy, listening to patients' fears, providing a sense of 
control and good written communication between staff. This report greatly improves the search for tangible psycho-educational care as it specifically states the areas in which intervention is required. It also recognises the need for a more formal approach to pre-operative psychological care. One further research study by Clipperley et al (1995) clearly identified the need for differing levels of information, differing kinds of psychological support and indeed provides the only brief formal pre-operative psychological careplan. Unfortunately, however, on closer inspection, even this plan does not precisely state what the nurse must do to secure effective psycho-educational support. It merely states, "The psychological component includes interventions which explore the patient's attitudes and feelings." (p. 203).

A principal reason why greater attention to psycho-educational intervention may not have occurred thus far may therefore, be associated with the absence of a formal pre-operative psychological programme of care i.e. no contemporary plan of the most effective way in which to deliver the appropriate psychological interventions (Linden et al 1996). For example, the physical nursing interventions required by a breathless, unconscious or immobile patient have been well documented, exist in formal programmes of care and can be easily taught and replicated whenever necessary. Nurses do not therefore have to guess what care to provide for the breathless, unconscious or immobile patient (Alexander et al 1994, Mallett and Bailey 1996, Nettina 1996). However, the lack of effective psycho-educational management plans both in general surgery and in day-case surgery has resulted in this very problem i.e. nurses guessing 
what psychological care to provide and delivering the supposed interventions on an ad-hoc basis. The lack of explicit pre-operative psycho-educational programmes is clearly evident in a number of recent nursing texts concerning day surgery (Edmondson 1996, Penn et al 1996, Markanday 1997, Hodge 1999, Meeker and Rothrock 1999, Malster and Parry 2000). These texts not only deal very briefly with the nurses' role in anxiety management but also contain no tangible elements of intervention other than recommending the provision of information. Additionally, no assistance is provided regarding the amount, level and type of information patients might require.

Ultimately, formal pre-operative psychological programmes of care do not exist because the interventions necessary to create such plans remain underutilised. The actions required to ensure effective pre-operative psychoeducational support have not been systematically assembled and presented in a clinically acceptable manner. The development of a more formalised approach to psycho-educational management in day surgery is crucial for the comprehensive management of patients and the future success of day surgery. Without good preparation patients cannot effectively care for themselves at home, either before or after surgery. Without the ability to achieve a good level of self-care, day surgery expansion will be limited. The extensive amount of time once associated with lengthy in-patient elective surgical procedures has disappeared. Nurses therefore no longer have the opportunity to speak with their patients later the same day or the following day as frequently once occurred. Additionally, a considerable amount of recovery now occurs away 
from the hospital and professional staff. As day surgery continues to grow, both numerically and in its surgical complexity, the issue of sub-optimal psychoeducational care will also grow. The most effective form of pre-operative psychological management within day surgery will therefore remain a challenge until a solution to this complex issue is uncovered and implemented.

In summary, the amount of day case surgery currently undertaken has increased as a result of the advances in surgical and anaesthetic practice. This has been broadly welcomed because of improved cost effectiveness and increased patient satisfaction i.e. decreased time spent in hospital. However, such advances have concentrated largely upon medical efficiency with little or no consideration for a major patient centred issue i.e. psycho-educational preparation. The causes of pre-operative fears have been well documented for many decades and much care has been recommended although unfortunately not implemented. Pre-operative psychological preparation has therefore remained static during the considerable rise and expansion in day surgery. Such preparation may have remained unchanged as the identified components for effective psycho-educational support have not been considered, systematically assembled and presented in a clinically acceptable manner. Completely eliminating all anxiety for all patients prior to day surgery may be an unrealistic aim. However, helping all day surgery patients manage their anxiety more effectively in the $21^{\text {st }}$ century is a very realistic and obtainable goal. Firstly, however, in order to help achieve this goal, the psychological perspectives concerning human coping must be considered. 


\section{ChAPTER 2}

\section{Coping with Surgery}

\section{Introduction}

Throughout the early part of the $20^{\text {th }}$ century the psycho-dynamic approach to coping was the main perspective put forward to explain how individuals manage an aversive event such as surgery i.e. 'work of worry' (Janis 1959). However, this approach was viewed as too narrow and not fully inclusive of all elements that may impinge upon recovery e.g. the hospital environment may be of considerable influence (Van Balen and Verdurmen 1999). Consideration of the patient / environment interaction or transactional approach therefore came to prominence during the later part of the previous century. Lazarus (1966) was one of the main instigators of this approach, which emphasised the dynamic appraisal of the situation rather than individual personality traits. However, it became clear from the plethora of studies examining psychological recovery from surgery that both individual and situational determinants were of equal importance. Therefore, towards the end of the last century Suls et al (1996) proposed a further coping perspective i.e. the convergent or combined approach. This approach was viewed as the 'third generation' of coping perspectives.

The above psychological approaches to coping will be examined more closely below for three main reasons. Firstly, because of their historical endurance, all require consideration when studying recovery from surgery. 
Secondly, many studies frequently give no mention of the psychological approach underpinning recovery from surgery. For example, numerous studies in this field of research have provided information booklets to participants, tested for knowledge of details contained within the booklets and subsequently commented on psychological recovery as a result of the knowledge scores obtained. The rationale for testing such knowledge or its association with an underlying psychological approach to coping is rarely provided i.e. how does such a knowledge score relate to the psychological recovery from surgery? The association is seldom explained as frequently the underlying aim concerns compliance with instructions and not necessarily psychological recovery. Thirdly, many studies have provided conflicting evidence as to the best method[s] of psychologically preparing patients for surgery. This may have arisen because a mixture of psychological approaches have been utilised. For example, many studies have employed a singular focus e.g. personality types (psycho-dynamic approach) and yet frequently utilise a transactional outcome measure e.g. Ways of Coping Checklist (Folkman and Lazarus 1980). Such practices may have helped to fuel the controversy concerning the most effective methods of pre-operative psychological preparation. When the psychological approach to coping is made explicit within the research paper, the mixing of the outcome measures i.e. outcomes not necessarily associated with the chosen psychological approach, gains greater transparency.

An examination of the broad psychological approaches to coping will be followed by studies, which have investigated more singular elements of 
psychological recovery. Such studies have demonstrated over many decades their influence on surgical convalescence. Finally, utilising the convergent or combined approach, the way in which patients might cope more effectively with modern elective day surgery will be put forward.

\section{Psycho-dynamic Approach to Coping}

Early in the $20^{\text {th }}$ century Sigmund Freud, Alfred Alder and Carl Jung first put forward the psycho-dynamic approach. This perspective provided the setting for the understanding of how people might cope with adversity. The management of anxiety was considered to be part of unconscious defensive mechanisms, which helped to protect the self from both internal and external conflicts. The type of coping behaviour, which subsequently arose from this approach, was rigid, reality distorting, unconscious cognitions, driven by past experiences (Suls et al 1996). One theory of coping utilising this approach was put forward by Byrne (1961) and termed 'Repression-Sensitisation' ('Personality Traits' section). "At one extreme of this continuum are behaviour mechanisms of a predominantly avoiding type (denying, repressing), while at the other extreme are predominantly approaching (intellectualising, obsessional) behaviours." ( $p$. 334). Byrne (1961) therefore suggested that individuals prefer to cope with threatening stimuli either by actively ruminating over events or completely avoiding all such thoughts (Diagram 1 p.20). However, many differing ego defence mechanisms were put forward by Sigmund Freud and the repressionsensitisation scale is considered to be just one of these mechanisms (Stevens 1989). Moreover, the repression-sensitisation scale was associated with the 
extremes of personality types, black and white behaviour, an all-or-nothing phenomenon (Suls et al 1996).

The work of Janis (1958) also pursued a psycho-dynamic theme. Janis (1958) conducted one of the first studies into the psychological impact of surgery and concluded anxiety was directly linked to the anticipatory fear associated with the physical trauma of surgery. He postulated the anxiety response could be reduced and recovery prospects improved if the patient thought about the adverse event and worked through their fear prior to surgery. He termed this type of pre-operative mental process as the 'work of worry' and believed it to be essential for reducing the stress associated with physical trauma. He described the 'work of worry' as a mental process, similar to mourning, which aided adjustment to a painful situation. If a patient were to undergo a surgical procedure he/ she would mentally rehearse the various situations together with their possible consequences. The benefit of this would be to have accurate expectations of the possible pain and discomfort and thereby have greater reality-based insight.

DIAGRAM 1

PSYCHO-DYNAMIC COPING PROCESS

INTERNAL THOUGHT PROCESSES i.e.repression/ sensitisation, ego defence mechanisms, work of worry.

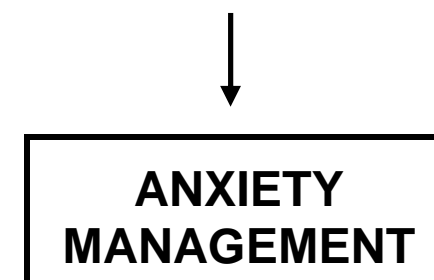


Individual levels of anxiety have, however, been viewed to differ among patients undergoing the same operation and not necessarily between patients undergoing major and minor operations, as implicitly suggested by Janis (1958) i.e. stress results from the physical trauma of surgery (Wolfer and Davies 1970, Ramsay 1972, Wallace 1984, Martelli et al 1987, O`Hara et al 1989). Wallace (1984) discovered even minor surgery to be stressful. This conceivably runs counter to the suggestions of Janis (1958), as the physical trauma of minor surgery should be less. Additionally, O'Hara et al (1989) studied 1420 patients undergoing differing types of surgery i.e. major and minor surgery, and stated "The findings suggest that, although patients may differ in many ways across types (e.g. in terms of sex ratios, severity of illness, and presence of cancer) there is no evidence from the present study that operation type is important in predicting level of post-operative distress." (p. 367).

Such studies possibly question the theory put forward by Janis (1958) whereby anxiety arises purely from the thought and degree of physical trauma. Differing levels of anxiety can be experienced with the same operation, not simply between the degree of physical trauma involved. Furthermore, the 'work of worry' may contradict other psycho-dynamic approaches i.e. repressionsensitisation (Byrne 1961), as some individuals (repressors), may prefer to deny or inhibit their stress evoking thoughts and not cognitively work through them as suggested by Janis (1988).

Anxiety may therefore be a reflection of the stress associated with the thoughts of undergoing surgery combined with the stress of the physical 
trauma, as reported in a review of the literature concerning psychological factors in surgical stress by Salmon (1992b). Several other studies concerning preoperative preparation also support the notion of both physical and psychological stressors. The main reported causes of pre-operative anxiety, put forward over many decades, are fear of being put to sleep, being unconscious, the pain and discomfort and the operation itself (Ramsay 1972, Ryan 1975, McCleane and Cooper 1990, Mitchell 1997, McGaw and Hanna 1998). Clearly, such a range of fears can have both a physical and psychological basis. For example, fear of being put to sleep (predominantly the most fearful aspect) may arise from the physical threat i.e. use of needles, mask over face, use of powerful drugs and also the psychological threat i.e. loss of control, not waking-up, embarrassment.

\section{Transactional Approach to Coping}

As a result of some of the contentious issues above i.e. degree of cognitive processing and essential anticipatory work, a second generation of coping strategies emerged in the 1960's. As an alternative to focusing purely upon internal and largely unconscious processes, the transactional model considered the interplay between internal and external processes. Lazarus (1966) conceptualised this type of coping as a process of change, which occurred between the person and their environment as opposed to coping behaviour resulting solely from personality traits and unconscious defensive mechanisms. Two constructs central to this approach are 'cognitive appraisal' and 'coping' (Lazarus and Folkman 1987). 
Firstly, cognitive appraisal is divided into 'primary' (initial thoughts) and 'secondary' (subsequent thoughts regarding ability to avoid, tolerate or ameliorate level of stress) appraisal. Folkman and Lazarus (1980) identified four types of primary appraisal - harm-loss (referring to the damage already occurred), threat (the anticipated harm-loss which may occur), challenge (the anticipated opportunity for mastery or gain) and finally, the possible benefit (Lazarus and Folkman 1987 later added this fourth feature). Secondary appraisal concerns the coping resources available to help master, reduce or tolerate the internal and/ or external demands created by the stressful transaction (Folkman 1984). "The degree to which the person experiences psychological stress, that is, feels harmed, threatened, or challenged, is determined by the relationship between the person and the environment in that specific encounter as it is defined both by the evaluation of what is at stake and the evaluation of the coping resources and options." (Folkman and Lazarus 1980 p. 223). As the situation changes so to does the individual's response. Coping is viewed as a dynamic process and determined by the exchanges between the person and his/ her environment i.e. primary appraisal may reveal a perceived stressor $\rightarrow$ secondary appraisal may involve the subsequent coping thoughts and actions to alleviate the perceived stressor resulting in $\rightarrow$ a positive outcome (Folkman and Lazarus 1980, Carver et al 1989).

The second construct in this approach concerns 'coping'. Two broad types of coping behaviour have been put forward in this approach i.e. problem focused coping and emotional focused coping (Folkman and Lazarus 1980, Folkman 
1984, Folkman et al 1986, Lazarus and Folkman 1987). Problem focused coping includes strategies in which the person attempts to directly challenge the stressor by embarking upon a physical plan of action. For example, when faced with the prospect of day surgery a patient may wish to discover exactly what will happen to him/ her, gain information about the operation, events on the day of surgery and the length of the recovery period in order to alter, circumvent or eliminate a particular stressor[s]. This type of coping has also been referred to as 'mastery' where the person tries to become better acquainted and more knowledgeable concerning the challenges confronting him/ her (Bandura 1977, Friedlander et al 1982, Mumford et al 1982, Folkman and Lazarus 1980).

Emotional focused coping refers to an individual's emotional attempts to deal with a stressor i.e. the conscious thoughts and feelings associated with the prospect of admission to hospital for surgery and general anaesthesia. For example, when faced with the prospect of an operation a patient may be able to cope more effectively, on an emotional level, if he/ she is aware that the latest equipment is to be utilised by an experienced surgeon, in a modern prestigious hospital using the most effective anaesthetic drugs (this type of coping has also been referred to as a 'cognitive coping') (Chapter 3).

The type of coping strategy employed i.e. problem focused or emotional focused coping, depends largely upon the individual appraisal of the situation (Lazarus 1966, Folkman and Lazarus 1980, Folkman et al 1986). If the stressor is deemed susceptible to change then problem focused coping strategies are frequently employed i.e. gaining as much information as possible concerning 
the operation, the possible alternatives, seeking out an experienced surgeon in a prestigious hospital, choosing a convenient date (Folkman et al 1986). If the stressor is deemed not to be susceptible to change then emotional focused strategies are more likely to be employed i.e. gaining assurance of improved health following surgery, talking with someone who has undergone a similar surgical experience (Folkman and Lazarus 1980, Folkman et al 1986). Emotional focused coping is therefore more akin to changing the cognitive response i.e. how an individual thinks and feels about a given situation, as opposed to the direct action associated with the problem focused approach (Diagram 2 p.24a).

\section{Convergent or Combined Approach to Coping}

The psycho-dynamic and transactional approaches can be broadly viewed as being internally and externally orientated, respectively, with a degree of flexibility. This either/ or approach has been considered too narrow and a combination of the two has been put forward. It has been referred to as the 'convergent approach' (Suls et al 1996). The need for a convergent approach has arisen as both internal and external factors have demonstrated their ability to impact, to a greater or lesser extent, upon human coping behaviour i.e. personality traits, physical environment, person/ environment interaction (Byrne 1961, Folkman and Lazarus 1980, Mumford et al 1982, Folkman 1984, Wallace 1984, Folkman et al 1986, Lazarus and Folkman 1987, Van Balen and Verdurmen 1999). 
From a psycho-dynamic viewpoint, individuals may cognitively appraise day surgery as more aversive, purely as a result of their internal influences i.e. past experiences, fear evoked by the thought of hospitalisation, fear of the unknown, fear of possible problems, individual traits. Their individual traits i.e. coping style, personality type, social cognitions, may also determine a desire to manage their anxiety in a particular manner. From a transactional viewpoint, individuals may evaluate the prospect of undergoing day surgery by their interactions with the hospital personnel and the environment in which the treatment is to take place i.e. opportunity to discuss planned surgery, grade of surgeon, perceived sources of stress, coping resources, reputation of the hospital, physical environment (Diagram 3).

DIAGRAM 3

CONVERGENT OR COMBINED COPING PROCESS

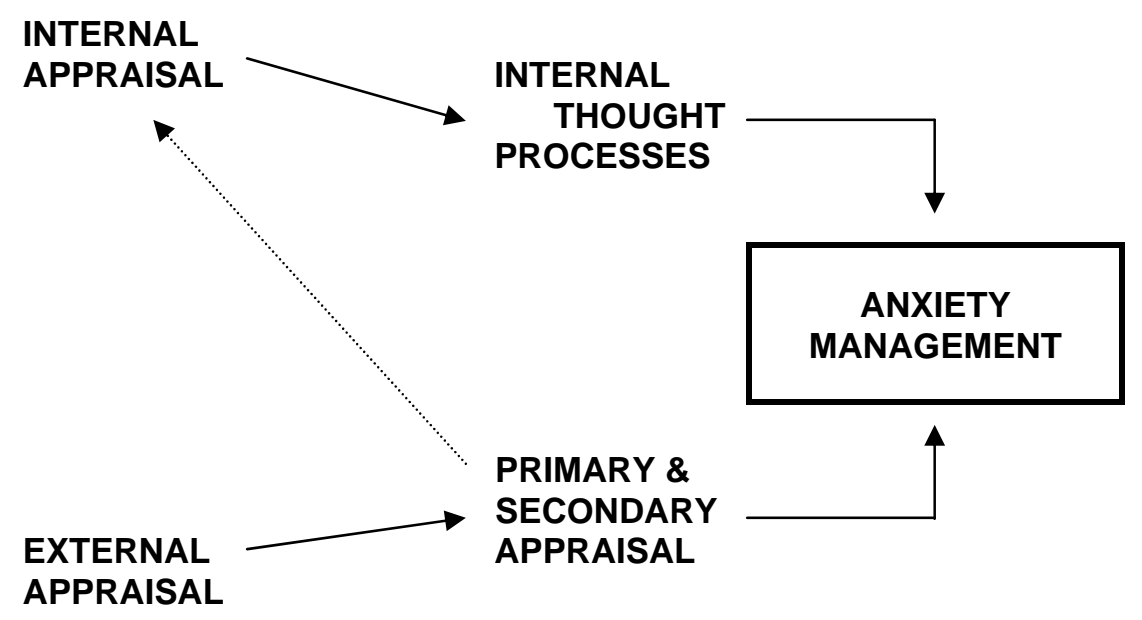

This combined approach will later be adopted in this chapter in order to help demonstrate how coping with modern elective day surgery may be enhanced. However, some singular approaches to coping with surgery will be considered 
next as they help to provide additional insight into future potential elements of psycho-educational support.

\section{Singular Approaches to Coping}

Over many years, a large number of studies have been undertaken in order to demonstrate how specific factors can influence post-operative outcomes (Mathews and Ridgeway 1984, Hathaway 1986, Suls and Fletcher 1985, Suls and Wan 1989, Devine 1992, Johnston et al 1992, De Groot 1997a). All the approaches utilised within the studies below have evolved directly from the psychological coping approaches already mentioned i.e. psycho-dynamic and transactional perspectives. However, while maintaining an underlying psychological coping approach (or not as the case may be), many studies have chosen a singular research focus. The large volume of studies helps to clearly illustrate i) the complexity of the issues surrounding recovery from surgery, ii) the diverse factors considered influential to successful recovery from surgery and, iii) why numerous suggestions regarding psychological recovery have been made with little subsequent progress. The specific areas to be considered are -
Personality
- neuroticism, vigilant and avoidant traits.
SOCIAL COGNITIONS
- health locus of control and self-efficacy.
SOCIAL SUPPORT
- degree of assistance from friends and family.
OPTIMISM
- level of confidence in achieving good recovery. 


\section{Personality}

\section{Neuroticism}

Ridgeway and Mathews (1982) believed the more anxious patient could be identified using measures of 'trait anxiety' and 'neuroticism'. Once identified, such patients could be given an increased level of psychological support to help improve their recovery prospects. In order to test this theory 60 participants undergoing hysterectomy were given some choice as to their preferred method of pre-operative preparation. No link between neuroticism and a desire for greater psychological support was uncovered. Indeed, some participants declined any type of preparation. No meaningful differences were observed on the neuroticism scale although the use of cognitive coping strategies was associated with a better recovery. Sime (1976) studied 57 patients admitted for abdominal surgery and gave no information pre-operatively. The participants were merely left to their own devices although their behaviour was closely observed. Some participants actively sort out information from the hospital staff and it was these subjects who had a better recovery. It was therefore concluded an unspecified personality variable associated with information seeking may have helped to ameliorate the negative effects of stress.

Manyande et al (1992) studied 40 participants undergoing minor abdominal surgery and provided some with relaxation therapy while others received procedural information i.e. told what will happen next or the sequential order of events (Chapter 3). The relaxation group were made worse by this type of intervention as it was concluded their 'work of worry` had been disrupted. If they 
had been left alone their stress reaction may not have been as great. Allowing patients to pursue individual methods of pre-operative preparation more suited to their personality type was therefore suggested as a far more effective method of preparation. Manyande and Salmon (1992) studied a further 40 participants undergoing minor abdominal surgery. The study was "designed to establish whether, in the absence of explicit programmes of study, active coping is correlated with a speedier recovery." (p. 228). It was concluded Type A behaviour and a feeling that one's health was independent of powerful others was related to an increased ability to cope.

Cohen and Edwards (1989) in a review of the literature identified three main perceptual buffers, which they believed helped to moderate the effects of stress i) personality factors such as hardiness and commitment, ii) social skills such as communication skills, talking, listening, and iii) intra-personal skills such as Type A behaviour, internal locus of control and coping style (vigilant and avoidant coping). Solcova and Sykora (1995) interviewed 32 patients undergoing dental treatment regarding pre-operative anxiety. Levels of trait anxiety and hardiness were established and those viewed as hardy had lower pulse rates and higher pain thresholds i.e. participants deemed as hardy experienced less anxiety and were more prepared for their surgery.

\section{Vigilant and Avoidant Traits}

One of the earliest studies undertaken concerning vigilant and avoidant personality traits was by Byrne (1961) in which he referred to this type of coping as the 'Repression-Sensitisation' scale. He suggested these two characteristics 
were on a continuum with the extreme forms at opposite ends. Repression consisted predominantly of avoiding, denying and repressing behaviours i.e. side-stepping any information, not thinking about the event and averting any thoughts. Sensitising behaviours consisted predominantly of approaching, intellectualising and obsessional behaviours i.e. persistently and relentlessly pursuing information. Furthermore, he stated "individuals are consistent in their defensive reactions to threatening stimuli over a period of time." (p. 336). Hock et al (1996) defined cognitive vigilance as the "...increased intake and exhaustive processing of threatening information" and cognitive avoidance as "...turning attention away from, and inhibiting the further processing of, cues associated with threat." (p. 1052).

Krohne (1978) referred to the repressor-sensitiser model as an anxiety defence mechanism. Later, Krohne (1989) gave the two coping modes slightly different titles i.e. cognitive vigilance and cognitive avoidance. An approach to and an intensified processing of, threatening information distinguishes vigilance. Its purpose is to help gain control over the main threat-related aspects of a situation thereby protecting the individual from the perception of unexpected dangers i.e. nothing surprises them as they are already aware of all the pertinent issues (Krohne 1989). Cognitive avoidance is defined as a withdrawal from threat-relevant information. Its purpose is to reduce the arousal caused by the confrontation with an aversive event (Krohne 1989). However, not all individuals were observed to maintain such extreme forms of behaviour. Therefore, two further aspects were put forward to create a two-dimensional 
model i.e. a vigilant and avoidant dimension, as opposed to one continuum. Fluctuating coping (variable information requirements) and situation-related or flexible coping (information requirements adaptable to the given situation) were added. Moreover, he put forward possible behaviours, which correspond to the individual coping style e.g. a rigid vigilant coper may repeatedly recall negative events, be consumed with self-pity and constantly seek information (Diagram 4 p.32).

Miller (1980) referred to this type of defensive coping as the 'Blunting Hypothesis` and used the terms 'monitors and blunters'. She stated blunting strategies included a positive side i.e. distraction, self-relaxation, reintellectualisation (reinterpretation of a given situation). According to Roth and Cohen (1986) this type of behaviour has its roots in psycho-analytical theories of development. Mechanic (1980) also suggested such defensive coping modes were behaviours learnt in childhood. Indeed, Miller et al (1995) suggests vigilant and avoidant behaviour occurs in children. Such ways of coping once learnt at an early age could become a habitual response in a stressful situation and may persist over time (Averill and Rosenn 1972, Krohne et al 1992, King et al 1998). 


\section{FREQUENCY OF VIGILANT \\ COPING STRATEGIES}

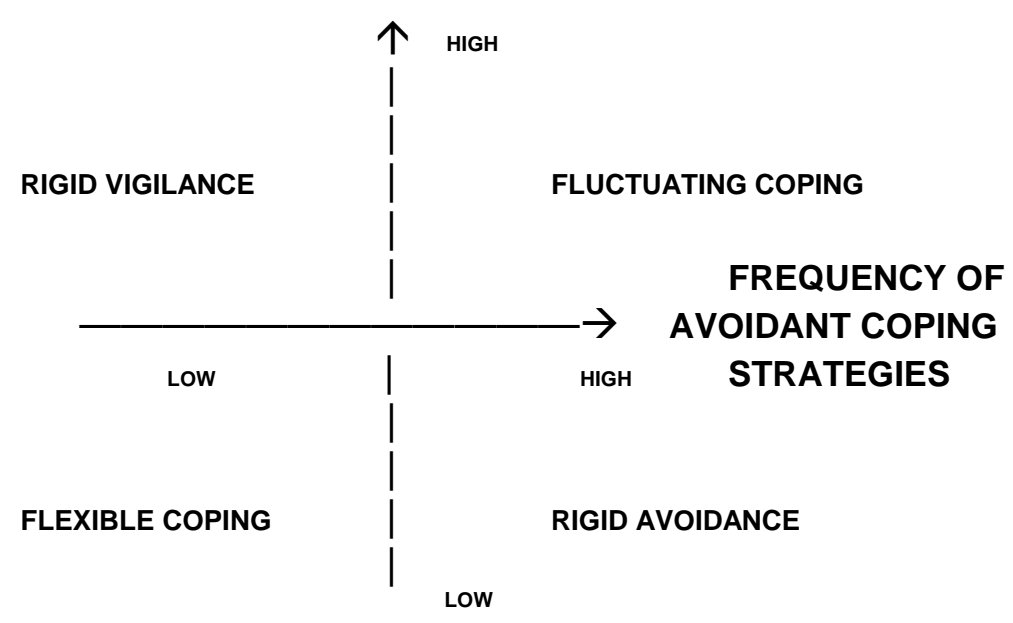

Vigilant Coping Strategies -

High vigilance

Low vigilance

Avoidant Coping Strategies -
1. Recalling negative events

2. Self-pity

3. Information search

4. Comparison with others

5. Planning for the future

6. Escape tendency

7. Control via information

8. Anticipation of negative events

9. Situation control
High avoidance
Low avoidance
High avoidance
1. Minimisation
2. Self-enhancement i.e. I'm fine and coping well
3. Re-interpretation i.e. positive interpretations
4. Attentional diversion
5. Playing down by executing incompatible reactions i.e. a sudden pain following surgery is normal and not an indication of a more serious problem
6. Denial
7. Emphasising one's own efficacy
8. Accentuating positive aspects
9. Trust i.e. doctors and nurses are very competent

(Krohne 1989) 
Mogg et al (1990) stated anxiety-prone individuals in acutely stressful situations might very easily activate their perception of threat relevant cues. Vigilant copers can go into 'cognitive overdrive' in an aversive situation, extracting information from their environment and processing it constantly as negative or threatening. Hock et al (1996) reported vigilant individuals could often make matters worse for themselves in stressful situations as they ".. often impose threatening interpretations on ambiguous event descriptions." (p. 1062). According to Bonanno et al (1991), this cognitive activation or cognitive overdrive involving the internal and external searching for cues requires little conscious effort. Highly anxious people can become extremely adept at picking up negative information with minimal conscious endeavour. Miller et al (1996) also suggests high monitors (vigilant copers) with a serious medical condition ruminate continually. "This increase in intrusive ideation (invasive negative thoughts) indicates that high monitors are more likely to think about their disease status when they do not mean to do so, to dream about it, to have trouble falling asleep because of it, to be reminded of it, and to have strong feelings about it." (Miller et al 1996 p. 221). "Intrusive ideation entails recurrent, reliving of the event with intense psychological distress when exposed to symbolic reminders of the event (including self-generated cues), as well as sleep disturbance." (Miller et al 1998 p. 848).

Cognitive avoiders according to Bonanno et al (1991) do the opposite in that "...repressors are associated with a general cognitive capacity for avoidant processing that can be invoked whenever the motivation to disattend is present, 
regardless of the specific source of that motivation." (p. 396). Hansen et al (1992) referred to the ability to avoid incoming information as 'attentional narrowing`. This becomes an effective perceptual defence or barrier to unwanted material. Hock et al (1996) also stated cognitive avoiders process (unconsciously in the initial stages), far less threatening information than vigilant copers. In a dental surgery study by Baume et al (1995), this was seen as advantageous as such patients were viewed as being less anxious during the procedure. However, Mullen and Suls (1982), Suls and Fletcher (1985) and Kohlmann et al (1996) postulate avoidance to be a disadvantage in the long term as all too frequently healthcare matters requiring attention may be ignored.

\section{Social Cognitions}

According to Conner and Norman (1996), two broad social cognition models have historically been applied in health psychology to explain health related behaviour - Attribution Theory (individual causal explanations and responses to treatment) and Predictors of Health Related Behaviour of which there are five i.e. health locus of control, self-efficacy, protection motivation theory, theory of reasoned action and health belief model. Two aspects of the predictors of health behaviour (health locus of control and self-efficacy) will be examined more closely here as the full casual explanations for ill-health and the need for surgery are beyond the scope of this study. Likewise, Attribution Theory will not be discussed as the focus of the present study lies with the experience of an acute surgical episode and not with the individual's interpretation of health breakdown. Additionally, health locus of control and self-efficacy are considered 
to be central indices in the predictors of health behaviour and many studies have utilised them as measures of health behaviour (Wallston et al 1978, Wallston and Wallston 1982, Wallston 1992, Oberle 1991, Antonovsky 1991, Cozzarelli 1993).

\section{Health Locus of Control}

Studies suggest an individuals' desire to be included in the decision making process regarding health may be related to their level of internal and external health locus of control (real or perceived). Rotter (1966) was one of the first researchers to examine and validate a questionnaire to help test claims of perceived control. The theory is based upon the assumption that 'internals' have a greater belief in their ability to shape their own destiny whereas `externals` feel more influenced by luck, fate and powerful others.

A number of studies have indicated that when a patient is given an increased amount of health control, adaptability and recovery are enhanced (Strickland 1978, Seeman and Seeman 1983, Peterson and Stunkard 1989, Bartlett 1990). In a review, Peterson and Stunkard (1989) concluded patients with increased appraisals of control were more likely to follow medical advice and be more skilled at coping. The review also asserts that control resides in the transaction between the individual and the environment i.e. is context specific and thereby open to manipulation. Therefore, although health locus of control may be considered a personality trait, external factors can influence such behaviour. Other studies have likewise indicated more favourable outcomes result from an increase in health control, as it has been positively associated with a reduction 
in the level of stress (Richert 1981, Mahler and Kulik 1990, Ludwick-Rosenthal and Neufeld 1993, Kugler et al 1994a, Litt et al 1995). Mahler and Kulik (1990) studied 75 males who were undergoing heart surgery, $85 \%$ of whom expressed responsibility for their own recovery. "...greater perceived control did marginally predict both lower pre-operative anxiety and fewer post-operative negative psychological reactions." (p. 748).

A greater level of control may provide a degree of predictability that removes, for some patients, the stress of the unforeseen event (Miller et al 1989). The level of health control required may only need to be minor or 'real or perceived as demonstrated by Mills and Krantz (1979) when giving blood donors a simple choice of which arm to use during the procedure. Which arm to be utilised mattered little to the hospital personnel but for the patient it bestowed the perception of choice. Miller and Mangan (1983) gave differing levels of information to 40 female surgical patients and concluded "One salient possibility is that information only decreased stress when it allows the individual to exert (or to perceive) some choice or control over the situation." (p. 232).

Manyande and Salmon (1992) studied 40 patients undergoing minor abdominal surgery who were purposely not given extra pre-operative information. It was concluded the patients classified as having a Type A personality or expressing an internal locus of control, displayed a superior postoperative state i.e. less pain, anxiety and negative symptoms. Pickett and Clum (1982) followed 59 patients undergoing cholecystectomy (removal of the gallbladder) dividing them into internal and external locus of control groups. 
Differing methods of psychological preparation were then employed i.e. cognitive distraction and relaxation. Cognitive distraction resulted in the lowest level of self reported anxiety and was positively correlated with an internal locus of control. Krause and Stryker (1994) hypothesised from a 1969 to 1971 National Longitudinal Survey of stress in middle-aged men that increased levels of stress might be buffered by increasing the level of control. Having an internal locus of control was viewed as a positive factor and an external locus of control as a negative factor.

If a degree of health control is not provided, anxiety can increase. Peerbhoy et al (1998), studying 30 patients undergoing orthopaedic surgery, concluded taking control had little real meaning, as "patient control over medical care appears to be a theoretical and professional construct." (p. 600). The patients viewed control as either a right to individualised care from the nursing staff or a reliance on personal 'willpower'. Information was required because the patients wanted to know what was happening in order to reduce uncertainty and retain dignity. It was not for the involvement in the decision making process. Other studies have also highlighted the limited opportunities within the healthcare setting to establish a measure of control. For the patient who desires some control in the healthcare situation this can lead to an increase in stress (Miller et al 1989, Malin and Teasdale 1991). Again, this indicates that the healthcare environment can prevent patients from exerting a degree of control i.e. external manipulation of a personality trait. For the patient who prefers a level of health control, this can be a cause for considerable concern. 
Although Rotter (1966) suggested locus of control beliefs could be viewed as a personality characteristic, Halfens (1995) demonstrated the possible dynamic status of health locus of control beliefs following hospitalisation. Sixty-two patients were interviewed before, during and after admission to hospital for surgery. It was concluded health locus of control beliefs changed when an individual was hospitalised, especially the powerful others aspect. "The findings of this study indicate that in new and ambiguous situations, health locus of control can react as a situation-dependent belief" (Halfens 1995 p. 165). Avis (1994) also alluded to such beliefs following a survey of 22 surgical patients. The questioning of staff by patients was minimal and they frequently expected to be told what to do. Likewise Johnston et al (1992) demonstrated how the perception of control could be manipulated by the addition of a few sentences on a routine appointment letter i.e. patients perceived greater health control once it was granted. Individual health control was therefore directed and not necessarily spontaneous.

The influence of powerful others on health locus of control beliefs will be discussed again in the methodological section as modern surgery expressly involves patients in an unfamiliar, time conscious environment, with little information, being instructed on what to do by professional staff all in uniform i.e. considerably powerful others. In such circumstances individual health control beliefs can be profoundly influenced and rarely spontaneous. Milgram (1974) in a classic study provided support for such a view whereby the presence and influence of a powerful person in uniform considerably inhibited 
individual expression. Additionally, the possible threat of harm i.e. surgery and general anaesthesia, can contribute to such an imposing environment. Collectively, such an environment could dramatically manipulate an individual's perceived ability to exert influence over events and may, as in the studies above, lead to situation-dependent beliefs i.e. coerced, temporary shift in their customary behaviour.

Other studies have recognised a mixed response to control indicating that not all patients require such involvement. In a study by Eachus (1991), data concerning health locus of control were collected from 88 nurses and compared with the health locus of control beliefs of the general public. It was confirmed patients may expect to be controlled, to some extent, by powerful others while in hospital i.e. doctors and nurses. Breemhaar and Van der Borne (1991) stated "Increasing the level of perceived control can lead to an increase in stress if this control brings with it demands, which the person concerned does not wish to meet or which he/ she cannot (or thinks he/ she cannot) meet." (p. 203). Folkman (1984) suggested having only a small amount of control did not always equate with an increase in stress and Miller et al (1989), Antonovsky (1992) and Avis (1994) all state control could involve relinquishing responsibility to another person, chosen by them as more competent. Therefore, if a choice of which surgeon to consult can be made, an element of control may be established. Smith and Draper (1994) suggest the hospitalised patient has a strong belief in the expertise and ability of the doctors and nurses i.e. powerful others, and is therefore frequently very willing to relinquish control to hospital personnel. 


\section{Self-Efficacy}

Self-efficacy or confidence in one's ability to behave in such a way as to produce a desirable outcome can give rise to considerable distress if these abilities are reduced (Bandura 1977). "By conjuring up fear-provoking thoughts about their ineptitude, individuals can rouse themselves to elevated levels of anxiety that far exceed the fear experienced during the actual threatening situation." (p. 199). As part of the social learning theory perspective, perception of self-efficacy can easily be enhanced or reduced by social interaction i.e. vicarious experience (simply observing what others do and copying), enactive attainments (positive thoughts generated from the accomplishment of a task) and verbal persuasion (Bandura 1982). In a review of the literature by Scheier and Carver (1992) in which the broad question of coping was examined, they state, "In our view, perceptions of personal efficacy are one very important source of favourable expectations for successful goal attainment ..." (p. 223).

In a study by Litt et al (1995) 231 patients undergoing dental extraction under local anaesthesia were studied. Participants were divided into four groups and given slightly differing methods of preparation i.e. standard preparation, premedication, relaxation, and relaxation and self-efficacy enhancement (told falsely they were able to relax well). The participants in the group where selfefficacy was enhanced had superior outcomes i.e. lower levels of self reported anxiety, increased feelings of self-efficacy and higher scores on behavioural adaptation (reported by the dental surgeon). "The results indicate that this (false feed-back via verbal persuasion and enactive mastery or falsely being informed 
of their skilful ability to relax) was an effective, and potentially clinically useful, means of enhancing self-efficacy appraisals and thereby improving coping." (Litt et al 1995 p. 455). Gattuso et al (1992) obtained similar results when studying 48 endoscopy patients. Participants were divided into four groups to either receive relaxation plus self-efficacy enhancement, relaxation, procedural information or the standard ward preparation. Participants who had undergone self-efficacy enhancement plus verbal persuasion had more positive outcomes i.e. improved behavioural and emotional outcomes. Cozzarelli (1993) studied 336 females undergoing termination of pregnancy under sedation. Following a brief explanation of the operation on the day of surgery each patient was asked to complete a questionnaire containing five sections - optimism, self-esteem, perceived health control, self-efficacy and depression. This battery of questionnaires was repeated on the 2 nd and 21st post-operative day. Within the conclusions Cozzarelli states "..one of the most important contributions of personality in the context of coping with stressful life events may be to help motivate individuals facing such events to exert appropriate and/ or continuous coping efforts by increasing feelings of self-efficacy." (Cozzarelli 1993 p. 1232).

However, counter claims to the success of self-efficacy appraisals were made by Manyande et al (1995). Fifty-one patients undergoing abdominal surgery were studied. Participants were divided into two groups, one receiving a 30 minute audiotape instructing them in the positive ways to deal with any problems they may encounter while the second group received an audiotape of general hospital information. Utilising a visual analogue scale, the positive 
imagery group experienced less pain post-operatively although venous blood analysis revealed higher levels of adrenalin and nor-adrenalin i.e. physiological increase in anxiety. It was concluded that although self-efficacy appraisals had increased, anxiety had not decreased with the provision of additional positive imagery. Providing preparatory information aimed at increasing self-efficacy could therefore potentially have the opposite effect to the one intended i.e. it could increase the stress response and potentially reduce self-efficacy.

\section{Social Support}

In a classic study by Spector and Sistrunk (1979), social support and social contact were viewed as beneficial prior to an anxiety provoking event. "The results suggest that anxiety reduction did indeed occur in the presence of other people. However, it was not the mere presence of others, but their actual statements of reassurance that caused the reduction." (Spector and Sistrunk 1979 p. 122). Such claims have also been supported within the healthcare situation. Hartsfield and Clopton (1985) conducted a study employing 60 female patients undergoing general anaesthesia for the removal of the gallbladder. Participants were divided into three groups - reassuring information (medical and psychological), self care instructions (post operative advice regarding diet, exercise, coughing technique, etc) and neutral information (description of a general hospital and admission procedures with no specific information regarding the operation). There were no significant differences in anxiety reduction resulting from the differing forms of information provided. Contact with 
visitors however, (including the researcher) led to a significant reduction in patient anxiety.

Holahan and Moos (1985), in a community based survey of 394 subjects regarding the effects of stress, discovered possible gender differences associated with social support. Participants were divided into a 'stress resistant group' and a 'distress group'. Both men and women were found to be able to deal with stress successfully although in differing ways. "Stress-resistant men are more self-confident, energetic, and ambitious, whereas stress-resistant women enjoy qualitatively better social support in their family setting." (p. 745). This suggests males who have a greater resistance to stress may draw more on internal strengths i.e. self-confidence and resolve, whereas stress resistant females may draw more on external strengths (greater degree of social support and contact). Sherman et al (1997) examined the available research concerning locus of control and gender differences over two decades and one of the conclusions relates to females and their social support networks. He states "Women appear to have larger social networks than males, be more communicative within their networks, and be exposed to more life events via these networks." (p. 244).

Salmon (1992b) also recognised the positive influence of increased social support for both males and females. He suggested focusing too much upon the patient pre-operatively could become a very difficult task and therefore the environment should be the main focus of attention i.e. providing the facilities for the patient to maintain some control and encouraging emotional support from 
the relatives. Stressful everyday factors were also seen to have some bearing upon the outcomes of an operation in a study by Liu et al (1994) concerning 30 patients recovering from dental surgery. The study suggested recent stressful life events had a negative influence on recovery i.e. increased additional stress at the time of surgery could be detrimental.

In a survey of 103 patients undergoing an invasive medical procedure and local anaesthesia by Foulger (1997), 32\% of participants stated they would have preferred a third person to be present. The patients were only in hospital for the day undergoing cardiac catheterisation (fine catheter passed into the chambers of the heart under local anaesthesia) but because of the associated anxiety, reduced amount of time in hospital, lack of opportunity or a combination, insufficient information was gained/ retained. In such situations the mere presence of the nurse has also been found to provide a great deal of comfort to the patient, even when merely holding the patient's hand (Leino-Kilpi and Vuorenheimo 1993, Leinonen et al 1996, Moon and Cho 2001). Fareed (1996) in a qualitative study of 8 patients in an acute hospital setting identified two main themes concerning feelings of being reassured i.e. internal (perception of the environment) and external (nurse being near the patient). The presence of a nurse again provided assurance of safety. Furthermore, Teasdale (1989) defined the concept of reassurance in three ways i) a state of mind - renewed confidence in something or someone, ii) a purposeful attempt to restore confidence in someone and, iii) an optimistic pledge given to someone in an 
attempt to guarantee safety. Such definitions may all necessitate the physical presence of a doctor or nurse.

Finally, in a study Kulik et al (1996) of 84 males and their roommates following coronary artery by-pass surgery, support from other patients was viewed as very positive. Patients who were placed in the same room as other males who had undergone the same operation, and were recovering well, had a better recovery. Taylor and Lobel (1989) described how people use such social comparison activities during stressful situations to aid effective coping with illhealth. Seeing someone who was less fortunate and seeing someone who was more fortunate served differing purposes. Downward evaluations appear to make the person feel better about their personal situation whereas upward contacts provided information valuable for potential self-survival and successful coping.

\section{Optimism}

Positive expectations or an optimistic outlook regarding an aversive medical event has been observed to have a considerable impact upon post-operative outcomes (Carver and Scheier 1994, Schweizer et al 1999). Strickland (1978), in a literature review of the association between locus of control and health related behaviour concluded that if a match between the patient's optimistic expectations and the actual events were achieved, outcomes could be enhanced. In a further review by Scheier and Carver (1992) coping and optimism were positively correlated with a problem solving approach and the acceptance of the reality of an uncontrollable healthcare situation. 
Johnston et al (1992) studied 71 patients undergoing rehabilitation dividing them into two groups. Participants in group one were sent a routine appointment letter whereas participants in group two were sent the same routine letter although with an additional few paragraphs designed to increase control and thereby raise expectations. On average the participants in the second group tended to be more satisfied with the information provided and have significantly higher levels of perceived control. The inclusion of a few simple sentences on an appointment letter was therefore viewed to be influential in recovery. Friedlander et al (1982) studying 109 patients on the eve of major surgery, reported higher levels of anxiety in participants who had received images of a very stressful event. It was therefore concluded the focus of preparation should concern positive images of recovery and not merely information provision.

Jamison et al (1987) studied 50 women undergoing gynaecological, laparoscopic day surgery. Self-reported measures of anxiety, mood, outcome expectations and physical symptoms were all monitored on the eve of surgery. Patients who anticipated higher levels of physical discomfort did indeed report greater discomfort and longer recovery periods. Flood et al (1993) in a comprehensive study of the powerful influence of expectations studied 348 male patients following benign prostatic surgery. Strong support for an optimistic view of recovery was established as patients with positive expectations prior to surgery had better short and long-term outcomes. In a study by Schröder and Schwarzer (1998) the coping resources and recovery rates of 248 patients undergoing cardiac surgery were investigated. Having an optimistic belief 
regarding the outcome of surgery was viewed as superior to all other aspects of coping.

From a biological viewpoint, a number of studies have suggested a pessimistic outlook can have a negative effect upon physiological functioning i.e. the immune response, rendering a patient more susceptible to ill-health and delayed healing. Kiecolt-Glaser et al (1995) compared 13 people caring for relatives with dementia with 13 other people matched for age and financial status. The participants encountering greater levels of stress caring for their relatives experienced significantly slower rates of wound healing. Scioli et al (1997) also reported lower 'hope' scores correlated with frequency and severity of illness. Segerstrom et al (1998) found T-helper cells (phagocytic white cells) to be greater in number in more optimistic individuals i.e. such patients had the potential for faster healing with fewer complications. Räikkönen et al (1999) found pessimistic and anxious patients to have elevated blood pressure and thereby be more susceptible to increased post-operative morbidity. Finally, in a review of the literature by Miller and Cohen (2001) concerning immune system functioning, only modest evidence was established for improved immune system functioning when pre-operative psychological programmes were employed. Notably, this review did not uncover any study with an acute surgical episode in modern surgery i.e. only more major types of illness and surgical intervention were deemed worthy of examination. The effect of reduced immune system functioning on brief modern surgical episodes may therefore be minimal or at least too small to be of any significance. 


\section{Convergent or Combined Psychological Focus for Day Surgery}

A convergent or combined approach to psycho-educational management in day surgery is required. Modern surgical practices have considerably reduced the amount of time the majority of patients spend in hospital when undergoing elective intermediate surgery. Contact with hospital staff is therefore considerably diminished leading to a lack of time in which to gauge individual psycho-educational requirements i.e. information, social support and health control. Additionally, time for the patient to visit the clinical environment prior to the day of surgery in order to undertake primary and secondary appraisal has almost vanished. Prior to day surgery patients were admitted to hospital 2 to 3 days prior to surgery and more able to familiarise themselves with their surroundings. A combination of the psycho-dynamic and transactional approaches to coping is now required in order to provide effective psychoeducational care in a new era of surgical intervention. The restrictions imposed by modern day surgery preventing the sole use of either the psycho-dynamic or transactional approaches will be discussed. This will be followed by an outline of how a convergent or combined approach may be more successfully integrated within modern surgical practice.

\section{Restrictions to Current Approaches}

The psycho-dynamic and transactional approaches provide insight into the origins of human coping during a stressful event. However, a combination of the 
two may help to direct future psycho-educational support in this new era of surgery. The singular approaches i.e. personality, social cognitions, social support and optimism, have also helped to sharpen the focus regarding the direction of explicit support. If tangible pre-operative psycho-educational interventions can be assembled utilising a combined psychological approach i) future psychological management may be greatly enhanced and ii) psychoeducational support may be better placed to keep pace with modern, elective, adult day surgery.

Modern surgery practices are inexorably leading to considerably shorter episodes in hospital. Medical practices have advanced greatly to make such developments possible. However, elective surgical healthcare is dominated by rapid anaesthesia and recovery, minimal access surgery (keyhole surgery), reduced nurse/ patient contact and considerable patient self-preparation and self-recovery. Psycho-educational aspects of care have become almost completely submerged in the wake of medical fervour associated with surgical and anaesthetic advances in day surgery. Until a more structured plan of information delivery and tangible aspects of psychological care have been identified, such practices will inevitably continue.

From a psycho-dynamic viewpoint, individuals clearly focus upon differing aspects when experiencing the adversity of day surgery as a result of personality differences and past experiences i.e. personality traits, social cognitions. Such personality differences have been studied extensively within the psychological approaches to coping and, in more detail, with the singular 
approaches to coping with surgery. Past experiences can provide greater insight into the whole day surgery experience and produce more insightful appraisals i.e. more accurate primary and secondary appraisals. As the average length of stay in day surgery is currently $6 \frac{1 / 2}{2}$ hours and individual requests for care restricted due to the medical domination of day surgery practice, a wholly psycho-dynamic approach to coping will be restrictive. For example, negotiating an individual level of information, establishing the rudiments of health control and gaining advice on coping once discharged are all too frequently confined to a mere few hours (or less) of fragmented discussion on the day of surgery prior to going into the operating theatre. Even if such aspects of psycho-educational care were to be discussed at the pre-assessment visit, the likelihood of the same nurse being available on the morning or afternoon of admission to continue discussions is negligible. Moreover, any attempt to enhance the psychological aspects of care on the day of surgery could easily be superseded by urgent medical interventions i.e. blood tests, surgical examination, consent gaining, anaesthetic examination.

From a transactional viewpoint, a number of issues may also prevent the adoption of a wholly transactional approach to coping. Patients stay in day surgery for a very brief period, the environment is completely unfamiliar and the hospital staff, although professional are almost always complete strangers. Therefore, a considerable amount of primary and secondary appraisal will be prevented. For example, if a patient has concerns regarding anaesthesia, the opportunity in which to encounter the environment prior to admission to discuss 
such concerns with the nurse and anaesthetist are extremely limited. Garden et al (1996) state the lack of time and unfavourable circumstances (pressure of the operating schedule) often have a detrimental effect upon the quality of anaesthetist/ patient communication. Badner et al (1990) and Moerman et al (1996) revealed anaesthetists were mediocre assessors of anxiety unless they had time to question the patient. If access to the day surgery environment is not possible and the surroundings in which surgery is to take place are unknown, effective appraisal cannot take place. Problem-focused coping strategies will therefore be largely curtailed if patients have little knowledge of the surgical environment and little advance opportunity to speak with the medical and nursing staff. As a result they will be unable to undertake numerous aspects of active coping i.e. gaining more information, knowledge of events on the day of surgery, planning for convalescence.

Emotional focused coping strategies may be viewed as the only available option as little can be undertaken on an individual basis to influence events. Being able to only utilise emotional coping strategies may be a source of great anxiety for some patients. Additionally, as emotional focused coping strategies rely on the re-interpretation of events, this form of coping could likewise prove ineffective. Patients are frequently unsure of the events to occur on the day of surgery, as they have commonly never previously visited the day surgery unit.

A new approach to help patients cope more effectively with day surgery must be established as the psycho-dynamic and transactional approaches can no longer function independently in the modern surgical arena i.e. brief admission, 
nominal nurse/ patient contact, rapid anaesthesia, minimal access surgery and rapid discharge. By embracing a more convergent or combined approach, psycho-educational support more suited to application in the modern surgical arena may be accomplished i.e. a realistic, formal psycho-educational plan, which encompasses both the psycho-dynamic and transactional perspectives. Such an approach may also provide a greater individual emphasis thereby allowing for a degree of choice and an improved opportunity for primary and secondary appraisal.

\section{Future Direction}

Coping can be broadly defined as the cognitive and behavioural attempt to master, reduce or tolerate the internal (emotional) or external (environmental) demands associated with a stressful transaction (Folkman 1984, Folkman and Lazarus 1980). However, situations may be perceived differently depending upon aspects of an individual's personality. Therefore, any comprehensive attempt to aid coping prior to day surgery must consider both the psychodynamic and transactional perspectives.

Firstly, consideration must be given to the main issues within the psychodynamic approach identified in greater detail within the singular approaches to coping with surgery i.e. personality and social cognitions. Such aspects can be broadly considered as being the internal influence. The most pertinent issues within this approach must become the central themes for future psychoeducational management i.e.

COPING STYLE $\quad$ - help to establish desired level of information. 
HeAlth locus of CONTROL APPRAISAL

SELF-EFFICACY APPRAISAL
- help to establish degree of healthcare control. - help to establish positive viewpoint.

If information provision is limited, as is clearly the case (Chapter 3), many patients will become very anxious e.g. vigilant copers, as the desired additional information may only be gained (if at all) in the few hours prior to surgery. Furthermore, information provision merely on the day of surgery may be too late for many patients. Information provided prior to surgery within the day surgery unit, the individual nurses caring for the patient and the time available for nurse/ patient communication therefore become strong determinants of the quality of information delivered. It cannot be assumed the desired level of information will be automatically provided by formal mechanisms within the day surgery unit or by the nurse in the brief time available. Such a psycho-dynamic consideration i.e. provision of information for individuals with differing coping styles, is frequently overlooked on the day of surgery as a result of numerous vital medical issues. Additionally, by not providing a choice of information, problem focused coping strategies may be weakened i.e. lack of knowledge concerning planned surgery and events on the day of admission. To compensate for this, formal mechanisms for receiving information prior to the day of surgery must be established and the provision of differing levels of information made a necessity, not an option. It must be accepted that patients have differing information requirements and that one level of information provision is no longer appropriate. 
Similar problems may ensue for patients who desire the rudiments of control over health issues. The modest time available, heavy medical focus and insufficient information concerning all events may negate all attempts to establish even minimal aspects of healthcare control. The powerful others element may dominate, irrespective of individual wishes and a perceived absence of choice may ensue. Again, if prior knowledge of the situation were to be made available, problem focused coping strategies could be more effectively utilised. For example, day surgery units could run teaching sessions, similar to antenatal classes for expectant mothers. If desired, patients could visit the unit at a pre-arranged time to discuss aspects of their care with staff, view a videotaped presentation, meet with other patients and thereby become more informed of their possible options. This may help to reduce the appraisal of powerful others and encourage the notion of choice, albeit a limited one. Indeed, some day surgery units in the United States of America can be viewed on a webcam, such is the desire for information and to preview the healthcare environment.

Likewise, self-efficacy enhancement may be extremely limited under the pressured circumstances of the operating schedule i.e. not being provided with the desired level of information, written or verbal or positive images of a good recovery. This may prove detrimental to an individual's appraisal of their ability to cope at home prior to surgery and once discharged. The secondary status of such individual wishes can only have an unfavourable effect upon self-efficacy appraisal. During the proposed optional pre-operative visit to the day surgery 
unit, outlined above, aspects of social, financial and personal arrangements could be discussed to enable the implementation of additional problem focused coping strategies in order to enhance self-efficacy appraisal.

Secondly, equal consideration must also be given to the main issues within the transactional perspective i.e.

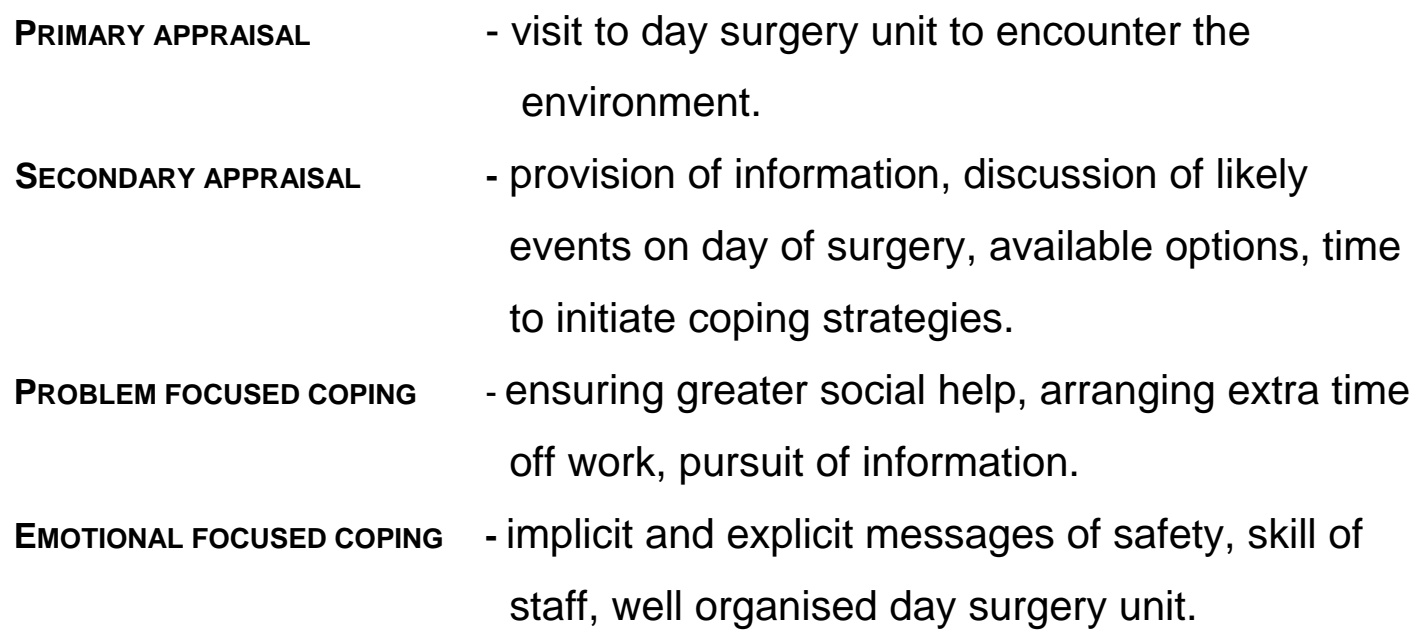

Enhanced primary and secondary appraisal could commence with the provision of pre-operative visits, meeting the staff prior to the day of surgery, the provision of a structured programme of information and adequate time in which to initiate problem focused/ emotional focused coping strategies. Frequently, the first experience a patient has of the day surgery environment is when they arrive on the morning of surgery. A pre-assessment check commonly occurs a few weeks or months prior to surgery. This mainly concerns checking physical fitness for surgery and frequently does not even occur in the day surgery unit. If prior contact were to be established in the form of a visit, open discussion with staff or virtual tour on a website, patients would gain greater insight into what is expected and hence experience more effective primary and secondary 
appraisal. This would give rise to more accurate problem focused coping strategies i.e. ensuring greater social help, arranging extra time off work, negotiating the desired level of information and health control. Enhanced emotional focused coping strategies may arise from the implicit and explicit messages of safety experienced during such an encounter i.e. well organised unit, efficient staff, and the interpersonal skills of the day surgery staff. Put simply, the patient would have greater insight into what was to happen and thereby more time to be more able to undertake (for them) the relevant individual problem focused and emotional focused preparations.

The overlap between these two approaches is where the psycho-educational support, required by day surgery patients, might therefore be found. It is the balance between what the individual desires in order to cope with the adverse situation and what is actually provided that becomes the decisive factor i.e.

- desired level of information required versus actual provision.

- desired level of health control versus the influence of powerful others.

- desired level of self-efficacy enhancement versus actual level provided.

- desired level of primary and secondary appraisal versus permissible opportunities.

However, it may be matching of coping style with the correct level of information that is the most important element as this has the potential to impact greatly upon appraisals of both health control and self-efficacy. For example, if a patient experiences raised anxiety in association with day surgery as a result of insufficient information i.e. vigilant coper (psycho-dynamic approach), and 
primary and secondary appraisal of the experience cannot fully take place until the morning of surgery (transactional approach), anxiety may remain increased, or become even greater. This may occur as the desired level of information has not been automatically provided and the related problem focused coping strategies (active pursuit of information) and emotional focused coping strategies (awareness of staff being helpful in providing information once admitted) cannot be effectively mobilised. Some secondary appraisal can obviously occur i.e. reliance upon previous healthcare experience, although for the majority of patients, prior contact with the day surgery unit is zero. The lack of desired information could have a 'domino effect' upon the perceived level of health control i.e. lack of insight may lead to ignorance of available options and increased perception of powerful others. Reduced information provision may likewise have a negative effect upon appraisal of self-efficacy i.e. lack of help and advice on how to cope at home in the hours following surgery (Diagram 5 p.57a).

The major overlapping aspects between the two psychological approaches to coping therefore provide the key areas for the future psycho-educational management of patients prior to day surgery. Acceptance of differing coping styles, desired elements of health control, required self-efficacy enhancement, permissible primary and secondary appraisal versus the extent to which the day surgery environment is perceived to meet such individual requirements is of paramount importance. 
In summary, coping ability has been identified utilising three differing approaches. The psycho-dynamic approach considers unconscious cognitions driven by past experiences, consistent over time and exerting a strong influence over an individual's ability to cope. The transactional approach considers the interaction between the person and their environment and emphasises cognitive appraisal (primary and secondary) and the resultant coping strategies (problem solving and emotional solving approaches) arising from the individual encounter. The third approach combines these broad internal and external influences and is therefore referred to as the convergent or combined approach. This approach may be more applicable in future, modern surgical healthcare situations, as little time is available in which to identify individual requirements or for adequate individual appraisal of the situation to take place.

Singular aspects concerning recovery have been investigated in great depth in a quest to uncover the most effective preparatory methods. Personality variables such as Type A behaviour have been examined in relation to preoperative stress although little conclusive evidence has been established. Contemporary research, however, has gained greater success with vigilant and avoidant personality traits. Such studies have indicated patient preparation for a stressful medical event should always involve matching the amount of information provided with the patients' preferred coping style i.e. providing more information for vigilant copers and less for avoidant copers.

Four further aspects considered to greatly influence recovery from surgery are health locus of control, self-efficacy, social support and optimism. Achieving 
some health control when required has been linked with more favourable outcomes and a reduction in anxiety. More positive views of coping has also assisted as patients frequently have little knowledge of what is to happen to them and consequently little time in which to consider how they might cope. Social support can also have a positive effect upon the post-operative recovery rate and an optimistic view of events is of clear benefit.

Greater flexibility within surgical healthcare delivery is required and may arise out of necessity as the modern surgical environment moves inexorably towards greater minimal access surgery and reduced hospital stay. If evidence can be uncovered to support the need for such elements of psycho-educational management within modern elective surgery a formal plan of psychoeducational intervention could be established i.e. acceptance of and provision for individuals with differing coping styles, consideration of health control and assistance in achieving positive self-efficacy appraisals.

However, one final challenging aspect remains - information provision. Information provision is central to coping in the modern surgical arena and implicit in the psychological approaches and singular aspects of coping outlined above. Information provision has been identified as a major national and international challenge to day surgery (Chapter 4). A vast number of studies have examined information provision because of its central role in surgical recovery. Therefore, because of its complexity, specific role in coping with surgery and future role in modern day surgery, information provision will be discussed separately and in greater detail in the following chapter. 


\section{Chapter 3}

\section{Information Provision Prior to Surgery}

The provision of information prior to an aversive surgical or medical procedure has been studied intensively in the search for more effective methods of preparing patients for surgery. The categories of information currently available will be considered here together with the contentious issues they raise as information provision is crucial to modern day surgery as i) a considerable amount of recovery takes place at home (61/2 hour average length of stay in day surgery), ii) certain forms of pre-operative information have been identified as being more beneficial than others (Suls and Wan 1989, Lea and Phippen 1992, Johnston and Vogele 1993) and, iii) the correct level of information may be central to the delivery of effective psycho-educational care.

Firstly, the most commonly utilised pre-operative information provided in the healthcare situation, aimed primarily at assisting problem focused coping will be examined i.e. procedural, behavioural and sensory information. The emphasis will then move towards information aimed more at assisting emotional focused coping i.e. cognitive coping strategies, relaxation and modelling (Wilson 1981, Mathews and Ridgeway 1984, Rothrock 1989) (Table 1 p.62). While it could be suggested cognitive coping strategies, relaxation and modelling also provide direct information useful for problem focused coping, the transfer of emotional focused coping strategies is more explicit e.g. being assured of safe anaesthetic practices, imitating positive recovery behaviour following viewing of a videotaped programme. In addition, a number of literature reviews have 
TABLE 1

PSYCHO-EDUCATIONAL INFORMATION CATEGORIES.

\begin{tabular}{|c|c|c|}
\hline Category & $\begin{array}{l}\text { Coping } \\
\text { Focus }\end{array}$ & Description \\
\hline $\begin{array}{l}\text { PROCEDURAL } \\
\text { OR SITUATIONAL } \\
\text { INFORMATION }\end{array}$ & \multirow{3}{*}{$\begin{array}{l}\sum \\
\| \\
1 \\
0 \\
0 \\
\alpha \\
0\end{array}$} & $\begin{array}{l}\text { The sequential order of events on the day of surgery } \\
\text { once admitted to the surgical unit i.e. what will happen } \\
\text { next and the order in which the events will occur. } \\
\text { Studies in the United States of America often refer to } \\
\text { this as Situational Information. }\end{array}$ \\
\hline $\begin{array}{l}\text { BEHAVIOURAL } \\
\text { OR ROLE } \\
\text { INFORMATION }\end{array}$ & & $\begin{array}{l}\text { The behaviour[s] or action[s] the patient is required to } \\
\text { undertake before, during or after the surgical procedure } \\
\text { i.e. adopting a certain position for the procedure, } \\
\text { keeping a limb elevated, gentle movements only, deep } \\
\text { breathing exercises, no lifting for } 6 \text { weeks, etc. Studies } \\
\text { in the United States of America often refer to this as } \\
\text { Role Information. }\end{array}$ \\
\hline $\begin{array}{c}\text { SENSORY } \\
\text { INFORMATION }\end{array}$ & & $\begin{array}{l}\text { The bodily sensations the patient is likely to experience } \\
\text { before, during or after the surgical procedure i.e. the } \\
\text { likely sensations of the drugs entering the body during } \\
\text { the initial stages of anaesthesia, degree and duration of } \\
\text { pain, medical equipment utilised in the immediate post- } \\
\text { operative phase. }\end{array}$ \\
\hline $\begin{array}{l}\text { COGNITIVE OR } \\
\text { EMOTIONAL } \\
\text { COPING } \\
\text { STRATEGIES }\end{array}$ & & $\begin{array}{l}\text { The positive thoughts a patient can draw upon in order } \\
\text { to gain assurance they will be safe, awake from their } \\
\text { operation, be unharmed and gain a full recovery i.e. } \\
\text { being told of the highly trained staff, many similar } \\
\text { operations performed without problem, fast effective } \\
\text { drugs, modern well maintained equipment, strict pre- } \\
\text { assessment to verify fitness. }\end{array}$ \\
\hline RELAXATION & & $\begin{array}{l}\text { Individual strategies of relaxation or planned programme } \\
\text { of relaxation techniques i.e. music therapy, simple } \\
\text { methods of distraction, hypnosis. }\end{array}$ \\
\hline MODELLING & & $\begin{array}{l}\text { Directly by actively imitating [behaviourally or } \\
\text { cognitively] the required or desired behaviour i.e. via a } \\
\text { real-life event, demonstration/ teaching, reading hospital } \\
\text { leaflets, websites, videotaped programmes and aspects } \\
\text { of the media. Indirectly by passively imitating } \\
\text { [behaviourally or cognitively] the required or desired } \\
\text { behaviour i.e. watching other patients. }\end{array}$ \\
\hline
\end{tabular}


suggested both cognitive coping strategies, relaxation and modelling to be the most effective forms of psycho-educational preparation as some patients do not wish to hear procedural, behavioural and sensory information details immediately prior to surgery (Wilson-Barnett 1984, Breemhaar and Van der Borne 1991, Teasdale 1995a). Therefore, each of the six categories of information must be discussed when considering pre-operative preparation although the term 'information provision' should more precisely be referred to as 'psycho-educational management' (as is the case in many American studies) as this describes the practice more accurately. Comprehensive problem focused and emotional focused coping strategies can be initiated once the relevant information has been gained from all six categories. Additionally, it should be noted that within the clinical environment little or no attention is given to the dichotomy between problem focused and emotional focused coping information provision.

Secondly, the contentious issues arising from the studies concerning information delivery will be discussed in a little more detail as some patients, for example vigilant and avoidant copers, may require differing levels of information in order to aid effective problem focused and emotional focused coping strategies. As day surgery patients have indeed been identified as a group requiring differing levels of information (Chapter 4), the final part of this chapter will examine information delivery i.e. indicators for information provision, mode of provision, the timing of provision and indicators against information provision. 


\section{Information Categories}

In reviews of the literature spanning two decades and concerning the most effective forms of information provision by Wilson (1981), Mathews and Ridgeway (1984), Miller et al (1989), Rothrock (1989), Suls and Wan (1989) and Johnston and Vogele (1993), six broad categories of information have been repeatedly highlighted as the most common i.e. procedural, behavioural and sensory information, cognitive coping strategies, relaxation and modelling. The terminology employed within these six categories has differed slightly over many years therefore the definitions, for the purposes of this study, are identified in Table 1 (p.62). In addition, many studies from the United States of America utilise differing terms to describe the same aspects of information provision i.e. procedural is described as situational information and behavioural as role information. The six forms of information can be broadly viewed as educational material aimed at assisting problem focused and emotional focused coping. Therefore each form of information provision will be discussed under these headings.

\section{Problem Focused Coping Information}

Many studies suggest problem focused coping information to be the most effective form of pre-operative information provision. This may result, as patients perceive, or they have been informed they can positively influence their recovery from surgery i.e. stopping smoking, losing weight, undertaking the recommended pre and post-operative exercises, arranging adequate time off work. If patients can therefore undertake problem-solving actions to improve 
their recovery prospects, problem focused coping information will be of great benefit. During the delivery of the six categories of pre-operative information, problem focused and emotional focused information can obviously overlap i.e. when gaining information regarding procedural events on the day of surgery (problem focused coping) a patient may benefit from the mere presence of the nurse (emotional focused coping) (Leino-Kilpi and Vuorenheimo 1993, Leinonen et al 1996, Moon and Cho 2001). However, for the purposes of theoretical explanation this classification will be employed. The three categories of problem focused coping information to be discussed are procedural, behavioural and sensory information.

\section{Procedural or Situational Information}

A number of studies have emphasised the importance of this form of information suggesting it to be one of the most important. Elsass et al (1987a) studied 81 patients undergoing surgery and divided them into two groups. Group one received procedural information while group two received a more detailed procedural account. Utilising subjective measures, the second group demonstrated lower levels of anxiety. Rothrock (1989) in a review of the literature concluded sensory and psychological information to be more effective for highly anxious patients while low anxiety patients benefited more from procedural information. Schoessler (1989) studied 116 patients undergoing various types of surgery and asked them what information they would most prefer. Psychological support, situational information (procedural) and role information (behavioural) were all rated as the most desirable forms. 
A mixture of approaches, as highlighted in the above study by Schoessler (1989), has also demonstrated positive effects. Suls and Wan (1989) in a review of 21 research papers concluded a combination of procedural and sensory information was the most effective method. Beddows (1997) studied 40 patients undergoing surgery and concluded both procedural and behavioural information helped to lower self-reported anxiety. One hundred and eleven patients in a study by Ziemer (1983) listened to an audiotaped presentation on the eve of surgery. Group one listened to procedural information, group two sensory information and group three - procedural and sensory information plus cognitive coping strategies (calming self-talk). However, no significant differences were established between the three groups.

In a study by Gammon and Mulholland (1996), 82 patients undergoing orthopaedic surgery were observed. Group one received a mixture of procedural, sensory and cognitive coping strategies while the control group received routine care. Utilising self-reported measures, the experimental group i.e. group receiving procedural, sensory and cognitive coping strategies, were less anxious and less depressed. It was concluded therefore that preparatory information was a behavioural guide, an aid to self-efficacy, and a point of convergence for emotional and problem solving strategies or a necessary part of the 'work of worry'. Finally, in a further review of the literature by Johnston and Vogele (1993) it was highlighted the most favourable outcomes were gained when both procedural and behavioural information strategies were employed simultaneously. 


\section{Behavioural or Role Information}

Lindeman and Van Aernam (1971) surveyed 261 patients undergoing surgery. All patients had their lung capacity measured and were then divided into two groups. The experimental group received a programme of helpful breathing exercises while the control group received no further intervention. The experimental group were observed to experience a significant reduction in the length of hospital stay. King and Tarsitano (1982), in a replication of this study, instructed 49 patients undergoing surgery on how to effectively cough and breathe in the post-operative period. Using entirely physiological measures the patients who had undergone the extra instruction had significantly better outcomes. In a study by Young et al (1994) 38 patients undergoing hysterectomy were contacted two weeks prior to admission to hospital. Participants were then randomly divided into two groups. Group one received a pre-admission teaching brochure containing specific exercise instructions deemed helpful for recovery, whereas group two received no teaching brochure. No significant differences on a satisfaction with care scale or an anxiety scale emerged.

Yount and Schoessler (1991) conducted a study in which 159 nurses completed a questionnaire concerning information provision. The answers provided were compared with the responses of 116 patients on a post-operative questionnaire regarding preference for information (Schoessler 1989). The nurses rated behavioural skills training, required for a speedier recovery, as the most important aspect to teach patients on the day of surgery. However, this 
was not the most important aspect for the patients on the day of surgery, as they believed psychological support to be of greater benefit. Oberle et al (1994) in a study of 294 day surgery patients also concluded nurses emphasised behavioural and sensory information provision on the day of surgery. In a study by Brumfield et al (1996) employing 30 day case patients and 29 nurses, the patients ranked behavioural information as the most important whereas the nurses ranked psycho-social support as the most important aspect of care.

\section{Sensory Information}

Miller et al (1989), in a review of the literature, concluded the most effective form of information for patients undergoing surgery or a stressful medical procedure was sensory information provision alone or in combination with procedural information. Hill (1982) studied 40 patients undergoing eye surgery and divided them into four groups. A seven minute audiotape of slightly differing information was shown to each group i) basic eye anatomy plus behavioural instructions, ii) basic eye anatomy plus sensory information, iii) both behavioural instructions and sensory information plus basic eye anatomy, and iv) general information. The group who received basic eye anatomy plus information regarding the likely sensations reported significantly less anxiety (Hill 1982). Hartfield et al (1982) studied 20 patients undergoing an unpleasant medical procedure (barium enema - radio-opaque dye inserted into the large bowel) although no anaesthesia was involved. Significantly less anxiety, regarding the likely sensations, was reported by the group who received sensory information as opposed to the group who received procedural information only. 
Padilla et al (1981) presented a teaching film to 50 patients prior to an unpleasant medical event, which again involved no anaesthesia (naso-gastric intubation for gastric content analysis). The group was divided into four and slightly differing information was provided i.e. procedural only, procedural with common distressful sensations, procedural with coping behaviours and procedural with coping behaviours to relieve the common distressful sensations. Patients reported the sensory and coping behaviour information to be the most effective in helping to decrease discomfort, pain and anxiety. Schwartz-Barcott et al (1994) studied 91 patients undergoing cholecystectomy and divided patients into three groups i.e. audiotape information, information from a nurse and routine care. The audiotaped information and the nurse both conveyed a mixture of sensory and behavioural information. Both these groups reported significantly less anxiety. However, routine care normally involves no extra care i.e. normal care received in that particular clinical environment. It is unknown what 'routine care' actually entails as it may differ from ward to ward (Auerbach 1989). Therefore, although utilised in numerous studies, routine care can frequently be a poor control.

\section{Emotional Focused Coping Information}

Although many studies suggest problem focused coping information to be the most effective, other studies have suggested emotional focused coping information to be of greater benefit. This may result from the perception of a healthcare situation in which stressors are deemed less susceptible to change i.e. the situation cannot be circumvented by planned problem focused coping 
action. Additionally, emotional focused coping strategies are possibly more likely to be employed if the provision of too much information causes an increase in anxiety i.e. participants utilising avoidant coping strategies. The three categories of emotional focused coping information to be discussed are cognitive coping strategies, relaxation and modelling (Table 1 p.62).

\section{Cognitive or Emotional Coping Strategies}

In a study by Ray and Fitzgibbon (1981) the first theory concerning recovery from surgery by Janis (1958) was investigated i.e. 'work of worry'. Emotional and behavioural outcomes were measured before and after surgery on 36 patients undergoing cholecystectomy. Patients with higher levels of preoperative arousal took fewer drugs, reported less pain and were discharged quicker. It was therefore concluded the mental rehearsal of the anxietyprovoking situation had been of benefit. Salmon (1993) also focused on the 'work of worry' by Janis (1958) and suggested preparation in the form of information provision may not always be in the patients' best interest. Alternatively, preparation should focus upon providing patients with the

opportunity to disclose their fears, receive assurance, social support and relaxation. Wallace (1986b) compared two studies concerning pre-operative preparation for surgery. Participants with increased pre-operative anxiety were more likely to have higher post-operative anxiety although this did not automatically result in a poorer recovery. Vogele and Steptoe (1986) studied 15 patients undergoing surgery and over a 2-day period collected both emotional 
and physiological data. It was concluded patients who thought about their operation more experienced reduced levels of stress.

In a review of the literature on alleviating the stress experienced by patients in hospital by Wilson-Barnett (1984), positive re-appraisals and information regarding possible sensations were seen as the most beneficial forms of information provision. Breemhaar and Van der Borne (1991) in a review considered the model by Lazarus (1966) to be the most effective approach i.e. problem focused and emotional focused coping strategies. It was suggested teaching patients mental strategies to aid coping i.e. teaching ways of distracting or emphasising any or all of the positive aspects of the surgery, could increase cognitive control.

In a review of hospital anxiety management by Teasdale (1995a) 'cognitive re-framing' was a highly recommended approach. Cognitive re-framing is the term Teasdale (1995b) uses to describe cognitive coping i.e. "...patients who use cognitive re-framing adjust themselves psychologically in such a way that events formerly perceived as threatening are now seen in a more positive light." (p. 81). Teasdale's (1995a) cognitive re-framing could therefore be considered very similar to emotional focused coping i.e. an individual's emotional attempt to deal with a stressor judged not to be susceptible to change (Lazarus 1966, Folkman and Lazarus 1980, Folkman et al 1986). Once in a given situation e.g. hospital ward prior to surgery, little problem focused coping can be undertaken therefore emotional focused coping may be utilised i.e. endeavouring to make a more positive cognitive appraisal of the situation. 
Pickett and Clum (1982) carried out a comparative study between relaxation strategies and cognitive coping strategies utilising 59 surgical patients. Cognitive distraction (a form of cognitive coping) resulted in the lowest level of self-reported anxiety. In studies by Miller et al (1989) and Krohne et al (1996) it was suggested patients who employed an avoidant coping style might have a greater preference for this form of preparation i.e. distraction or positive suggestions, as they generally choose to ignore cues in aversive situations or have more positive appraisals of all events in comparison with vigilant copers.

Finally, a number of more recent studies have increasingly employed spiritual aspects of coping in pre-operative preparation (Wallston et al 1999). Religious coping has been previously associated with social support (Krohne et al 2000) although it will be discussed in this section as other studies have viewed such coping as a form of cognitive or emotional coping. In a study by Tix and Frazier (1998) the religious coping strategies of 58 patients and their partners following Kidney transplantation were investigated e.g. prayer, church attendance and importance of religion. Such behaviour was associated with better adjustment although it was moderated by differing spiritual beliefs i.e. appraised differently depending upon the individuals' religious orientation.

\section{Relaxation Information}

A number of studies have advocated relaxation programmes to be a more beneficial form of anxiety management (Goldmann et al 1988, Caunt 1992, Salmon 1992b, 1993, Markland and Hardy 1993, Schwartz-Barcott et al 1994). In a study by Wells et al (1986), 24 patients undergoing surgery were divided 
into two groups. One group was provided with 'routine' ward preparation while the experimental group were taught relaxation techniques. The experimental group spent fewer days in hospital and required less analgesia. Holden-Lund (1988) studied 24 patients undergoing surgery and randomly allocated them into two groups. One group received an audiotaped series of relaxation while the control group were merely advised to have 'quiet periods'. The audiotapes were 20 minutes in duration and were administered once in the afternoon prior to surgery and again once per day for three days in the post-operative period. Using subjective and objective measures the experimental group had lower levels of anxiety and their surgical wounds presented with less erythema (reduced inflammation and quicker healing). Miro and Raich (1999) studied 92 patients undergoing general anaesthesia for hysterectomy. Participants were randomly divided into two groups - an attention control group and a relaxation group. The experimental group received 30 minutes of relaxation, one week prior to surgery and were also provided with detailed instructions on how to practice the techniques at home. All participants were tested for preferred coping style i.e. vigilant or avoidant behaviour, the assumption being avoidant copers would experience less pain when relaxation techniques were utilised pre-operatively. However, all participants in the relaxation group, irrespective of coping style, reported less pain and were more active in the post-operative period.

Music therapy demonstrated significant positive results in a study of 41 surgical patients by Augustin and Hains (1996). Although the experimental 
group only listened to the music for 15 - 30 minutes, subjective data collection revealed a significant reduction in anxiety. Cruise et al (1997) studied 121 patients undergoing sedation for eye surgery and assigned patients to one of four conditions i.e. audiotape of relaxing suggestions, normal quiet environmental noise, audiotape of previous operating room noise (during the same surgical procedure) or relaxing music. Utilising a visual analogue scale to gauge satisfaction, patients were more satisfied when listening to music although they were not less anxious. Finally, Domar et al (1987) studied 42 patients undergoing skin surgery and provided a 20 minute audiotape of relaxation for half of the group while the other half were asked to read a book of their own choice for 20 minutes each day. Although the programme of relaxation commenced 3 to 4 weeks prior to the day of surgery no significant subjective differences were established.

\section{Modelling Information}

Modelling of behaviour can be direct or indirect i.e. occurring as part of the individual's personal experience of the world or viewed indirectly via the media, friends or relatives. This can also be said of cognitive coping and relaxation strategies. Donovan and Blake (1992) suggest the media to be a huge source of information for the public and Thatcher (1996) stated the increased anxiety associated with hospital admission could stem, in part, from media coverage of such events. A large volume of medical reporting from sources as wide ranging as women's magazines to quality daily broadsheets, tabloid newspapers and television programmes can be observed on a weekly basis e.g. Sunday Times 
Hospital League Tables $14^{\text {th }}$ and $21^{\text {st }}$ January, 2001, Mail on Sunday Good Hospital Guide 10 ${ }^{\text {th }}$ March, 2002.

Indirect teaching can occur via videotaped or audiotaped presentations. Gagliano (1988) in a review of 25 research papers regarding the use of videotaped presentations concluded such programmes were of great benefit as patients often viewed a good role model or a patient with positive outcomes. Taylor and Lobel (1989) concluded that seeing positive comparisons during stressful situations might help some patients cope more effectively. Shipley et al (1978, 1979), Kempe (1987), Tongue and Stanley (1991), Heinen and Melodie (1992) and Leino-Kilpi and Vuorenheimo (1993) all concluded viewing a videotaped presentation prior to an aversive medical event to be of benefit to some patients. It helped to reduce the fear associated with the unknown elements, was cheap to run and did not require the patient to have a certain level of literacy skill, as with written information.

Wicklin and Forster (1994) in a study of 91 patients undergoing surgery compared the effects of two differing videotaped presentations. Although no significant differences were established the more anxious patients were identified. Finally, Mahler et al (1993) showed a 40-minute videotaped programme explaining recovery from extensive surgery to 127 patients. An increase in the appraisal of self-efficacy was observed following viewing. This was strongly related to reduced anxiety and an increased sense of mastery i.e. better acquainted with, and more knowledgeable regarding, the challenges confronting them. 


\section{Information Delivery}

As alluded to at the start of this chapter many contentious issues have arisen regarding information provision. One major issue concerns the classification of information provision i.e. information provision to assist problem focused and emotional focused coping (Table 1 p.62). Problem focused coping information is the main form of pre-operative information provision although no distinction between the two forms of information exists in practice and little choice regarding the form received is provided.

In this next section the contentious issues relating specifically to the delivery of all information in both information categories will be discussed i.e. indicators for information provision, mode of provision, timing of provision and indicators against provision. These issues are more pronounced when providing preoperative information aimed more at problem focused coping i.e. leaflets, verbal explanations, videotaped presentations. However, such issues are still of concern for information provision aimed more at the emotional focused aspects of coping with surgery i.e. cognitive coping strategies, programmes of relaxation and modelling.

\section{Indicators for Information Provision}

For many years a considerable number of studies have highlighted the lack of information provision during hospitalisation to be a source of stress. In a metaanalysis of 68 studies regarding information provision prior to surgical intervention, Hathaway (1986) revealed patients who received pre-operative instructions not only had more favourable outcomes but also the effects were 
$20 \%$ better than those not receiving pre-operative instruction. The Health Service Commissioner for England, Scotland and Wales (1997) highlighted five main areas of complaint against the National Health Service, three of which related to problems of communication. The Commissioner stated "The importance of communication has been emphasised repeatedly in previous Annual Reports and I make no apology for returning to it this year." (p. 5). This has been reiterated in the Health Service Commissioner for England, Scotland and Wales Report (2000/1). Four main areas of complaint emerged with communication with relatives being the first issue.

Volicer (1973) concluded communication difficulties increased stress while in hospital. Volicer and Bohannon (1975) produced a Hospital Stress Rating Scale, which incorporated the most stressful aspects of hospitalisation. "... some aspects of the experience of hospitalisation which are perceived as very stressful by patients are related to a lack of communication of information or lack of communication in a meaningful way..." (Volicer and Bohannon 1975 p. 358). Wilson-Barnett (1976) noted that $25 \%$ of hospital patients mentioned 'feelings of unease' regarding the lack of information concerning their medical condition. Wilson-Barnett produced a list of the top ten most positive aspects of hospitalisation and revealed four related directly to communication i.e. talking to the staff nurse, the student nurses, charge-nurse and visitors.

Wilson (1981) studied 70 elective surgery patients on the eve of their surgery. Numerous measures of data collection concerning recovery were employed including length of hospital stay, level of morbidity, self-rated recordings of 
mood, stress and physical recovery rate, and biochemical analysis of urine for cortisol to monitor emotional arousal. It was concluded patients benefited both from relaxation and information although in differing ways, depending upon their coping style i.e. vigilant and avoidant coping. In a survey of 150 patients by Ryan (1975) concerning pre-operative fears, 84\% reported increased feelings of anxiety, $30 \%$ were fearful of a diagnosis of cancer (even when a benign diagnosis had been given), 25\% had anaesthetic fears (42\% death under anaesthesia, 31\% lack of anaesthesia, 13\% waking up during the operation, $13 \%$ post-operative nausea), $17 \%$ feared the operation itself and $9 \%$ feared the possible post-operative pain. Ryan (1975) concluded the value of information prior to general anaesthesia regarding surgery, should not be underestimated. Ramsay (1972) in a comprehensive survey of pre-operative fear stated a calm patient who had been given some explanation was easier and safer to anaesthetise. Seventy-three percent of the patients included in the study had pre-operative fears relating to the anaesthesia i.e. not waking up, induction of anaesthesia with a mask, waking during surgery. Sixty-two percent of patients were concerned with pain during the operation, $15 \%$ surgical fears and $23 \%$ miscellaneous fears i.e. a diagnosis of cancer, being naked on the operating table.

Meredith (1993) conducted a comprehensive study in which 30 patients and 57 doctors and nurses were interviewed. Patients were firstly surveyed in the out-patient department and then post-operatively, in the ward. Time constraints in the out-patient department, the inappropriateness of doctor's ward rounds for 
intimate or serious discussion, the lack of involvement of the relatives in communication and other patients being in constant earshot were all highlighted as serious barriers to effective communication. Moreover, "In the face of the threat of a public airing of often acutely personal details, some patients will resort to the defence of saying as little as possible to anyone. Not to ask questions of staff becomes a means of avoiding public disclosure of personal information." (p. 598). In a study by Strull et al (1984) of 210 patients, again in an out-patient setting, $41 \%$ would have preferred more information while $58 \%$ said they had received the correct amount. It was concluded while much information was required, it was for information sake only and not necessarily an indication of the desire to be more involved in the decision making process.

Leino-Kilpi et al (1993) in a literature review of patient information provision highlighted two main educational approaches to patient education i) ideological - the patients fundamental right to know about their care and treatment and, ii) practical - the need for patient compliance with the prescribed treatment. In a study by Avis (1994) 22 surgical patients undergoing both general and local anaesthesia were interviewed. It was evident from this study that current educational ideology may lie more within the practical compliance domain than in the patients' fundamental right to know. "Although patients criticised the lack of information, and expressed a desire for more, they made surprisingly few attempts to question hospital staff. They expected to be told what to do and adopted the role of recipient rather than partner in care." (Avis 1994 p. 294). 
Redman (1993) in an analysis of nursing textbooks regarding patient education stated the delivery of health education might not always be to an acceptable standard. This may result from patients' impressions of the nurses being too busy or the emphasis resting more with the medical information at the expense of nursing information (Fleming 1992, Cortis and Lacey 1996). In a study by Cortis and Lacey (1996) 1,544 recently discharged patients were sent a questionnaire relating to satisfaction with hospital information. One of the conclusions related to patients viewing the nursing staff as too busy to help. "This resulted in patients not asking as many questions as they would have liked, and not expecting staff to have time to talk things over in any detail." (p. 680). This again may reflect a practical ideology towards information provision by both staff and patients i.e. the perceived desire for patient compliance (Leino-Kilpi et al 1993).

Discharge information was viewed by a number of studies to be of particular help. In a study by Bostrom et al (1994) in an acute hospital setting, 76 inpatients and 89 recently discharged patients were surveyed. The results demonstrated patients consistently required similar information i.e. advice on activities of daily living, skin care and feelings concerning their condition. The first two weeks following discharge were seen as the most important time as the patients were often striving to regain greater healthcare autonomy. It was recommended to establish a telephone helpline for patients to contact the hospital at anytime, an automatic follow-up telephone service, written discharge information, an unbroken system of communication between the hospital and 
community teams and a database for the nurses to help inform patients. Also, Bubela et al (1990) following a survey of 301 patients admitted to hospital with an acute medical/ surgical condition, concluded information enhanced the quality of life and encouraged positive feelings of recovery.

This and other evidence has led to the utilisation of telephone discharge services. It was revealed in a medical survey by Mukumba et al (1996) that during a 16 month period 939 telephone calls were made to their day surgery unit. Forty percent of these callers requested more information. Therefore, many patients were obviously leaving hospital unprepared for the events that lay ahead during their recovery period. Support for this view was also provided by Bostrom et al (1996) who conducted a telephone follow-up study utilising 1,400 recently discharged patients. For each patient it was documented in their medical records that they had received a great deal of information prior to discharge. Participants were divided into three groups - no intervention or encouragement, encouraged to utilise a nurse-run telephone service and nurse initiated telephone call service. Nine calls were received from patients in the group encouraged to use the nurse-run telephone service and 445 calls were made to patients on 2 or 3 occasions in the nurse-initiated group. It was revealed the information received by patients while in hospital had either been forgotten or not understood as more than $90 \%$ of the patients contacted by telephone during the nurse initiated calls $(n=445)$ had questions relating to their recovery. It was therefore recommended to establish an automatic followup telephone service and a database for the nurses to help inform patients. 


\section{Mode of Information Provision}

Several studies have highlighted the conflicting views regarding mode of information delivery i.e. written communication, verbal communication and videotaped presentation. Each of these modes of information delivery will therefore be examined commencing with written information.

Wallace (1984) studied 80 women having elective surgery and established that patients given detailed written information regarding their laparoscopic surgery had better outcomes via an enhanced level of empowerment. Wallace (1986a) also reported a study employing 63 women undergoing minor gynaecological surgery in which one group received no information, a second group received a minimal information booklet and a third group a maximal information booklet. When questioned 2 hours prior to surgery regarding knowledge of surgery, misconceptions, worries and information received; a mean difference in scores between the groups supported the use of a maximal information booklet. Law (1997), in a study of 38 day surgery patients two days after their operation, revealed $34 \%$ of patients could not remember what the doctor had said and $31 \%$ could only remember basic information. A leaflet for reference purposes was therefore viewed as invaluable to aid the management of a forgotten or unforeseen event occurring at home.

Conversely, some studies have suggested written information to have little impact and have recommended verbal information. Young and Humphrey (1985) studied 30 women undergoing hysterectomy and gave some written information and others verbal information. Using a combination of behavioural 
and psychological measures it was concluded a booklet was of no more benefit than verbal information. Young et al (1994) studied 38 women undergoing hysterectomy sending some patients a booklet prior to admission while others received no booklet. Behavioural measures of physical recovery in hospital were recorded and participants were asked to complete satisfaction, stress and social recovery questionnaires. Again, no significant differences were established between the two groups.

Furthermore, the quality of leaflet production within hospitals can be poor thereby reducing any positive effects the information may provide (Audit Commission 1993, Coulter et al 1998, Walsh and Shaw 2000). Scriven and Tucker (1997) examined 184 educational leaflets from 97 hospitals in England regarding information for women undergoing hysterectomy. The leaflets from 27 hospitals were found to be illegible, mainly as a result of being hospital produced photocopies.

The final mode of presentation concerns videotaped programmes. Gagliano (1988) in a literature review of the strengths and weaknesses of videotaped programmes concerning patient education reviewed 25 studies, all of which utilised videotapes. It was concluded videotapes were economical, easy to stop and rewind for repeated viewing, able to reach a wide audience and did not depend upon the literacy skills of the listener. Leino-Kilpi and Vuorenheimo (1993) studied 30 patients undergoing orthopaedic surgery and $50 \%$ stated they would have preferred to view a videotape of the procedure prior to admission. Doering et al (2000) studied 100 patients undergoing hip replacement surgery 
to evaluate the utility of a videotaped educational programme. The videotape was 12 minutes in length and was narrated by a patient who had undergone the surgical procedure explained events. The videotaped presentation was broadly aimed at the anticipation of events with some additional procedural information. The group were randomly divided into two i.e. control and experimental groups. The experimental group viewed the videotape on the eve of surgery while the control group received no viewing. Utilising behavioural, emotional and physiological measures the experimental group had lower anxiety although only when utilising physiological measures.

\section{Timing of Information Provision}

Although the number of patients undergoing intermediate elective in-patient surgery is diminishing rapidly, such patients have an increased amount of time (relative to day surgery patients) in which to gain information and glean answers to questions to allay fears. Prior to a decrease in the amount of time patients now spend in hospital, timing of information provision did not therefore necessarily present as a problematic issue i.e. the days spent in hospital during the pre-operative phase were frequently utilised to educate the patient. However, as the length of hospital stay has fallen and the amount of elective day surgery has risen, the issue of timing of information provision has gained momentum.

According to Johnston (1980), following her study of 136 patients undergoing various types of surgery, increased anxiety is experienced many days and/ or weeks prior to surgery and for at least five to six days following surgery. On an 
individual basis the exact time when anxiety begins and when it eventually falls has not been clearly established. Pre-operative anxiety is therefore not a minor, short-term emotional disturbance. It results from the rational fear of a serious life-threatening event and can last for many days/ weeks before and after surgery (Johnston 1980). Wallace (1987) interviewed 118 surgical patients who were requested to complete the State-Trait Anxiety Inventory (Spielberger et al 1983) during an out-patient visit and also prior to hospitalisation. An analysis of the results revealed elevated state anxiety scores six to eight weeks prior to surgery regarding their planned hospitalisation. If pre-operative anxiety does indeed begin in some patients many days/ weeks in advance of surgery and continue for many days afterwards, timing of information provision must be viewed as a challenging issue for day surgery. It must be regarded as a challenging issue for day surgery for two main reasons i) little time is available on the day of surgery for educational clarification, and ii) nurse/ patient and doctor/ patient contact in the pre and post-operative stages is very brief.

Christopherson and Pfeiffer (1980) conducted a study in which 41 patients undergoing cardiac surgery were either sent no information (group 1), an informational booklet 1 to 2 days prior to surgery (group 2) or an informational booklet 3 to 35 days prior to surgery (group 3). The level of patient anxiety and knowledge concerning surgery was measured using a self-rated questionnaire. Group 3 experienced the lowest anxiety pre-operatively and group 2 experienced the lowest anxiety post-operatively. However, this paradox may have arisen as a result of two design issues. Firstly, the exact time each 
member of the two groups read the information can only be assumed. Many participants in group 3 could have received the information 3,4 or 5 days prior to surgery and read it immediately. Many in group 2 who received the information booklet 2 days prior to surgery could have also read it immediately. The difference between receiving and reading information 3,4 , or 5 days prior to surgery and receiving and reading information 2 days prior to surgery may be indistinguishable. Secondly, the information booklet sent to participants in groups two and three was 16 pages long. From a practical viewpoint, patients in group 2 may not have had sufficient time to read the entire 16-page booklet 48 hours prior to surgery.

Schoessler (1989) conducted one of the earliest studies on preference for timing of information provision with regard to modern surgical practices i.e. patients admitted to hospital on the morning of surgery. Data were collected from 116 patients undergoing various surgical procedures and general anaesthesia. It was discovered that $50 \%$ of participants required information on admission, $41 \%$ wanted the information prior to admission and some merely wanted the information immediately prior to surgery. Furthermore, the information most requested prior to admission was psycho-social support i.e. how to cope on the day of surgery and afterwards at home. Yount and Schoessler (1991) compared the information from the above study (Schoessler 1989) with a survey employing 159 surgical nurses. The nurses agreed information should be provided prior to admission. 
O'Hara et al (1989) following a comprehensive study of 1,420 undergoing various surgical procedures concluded anxiety was greatest the day of surgery and post-operatively had no sudden end. Indeed, $14 \%$ of participants reported high levels of psychological distress up to three months after surgery. Cupples (1991) studied patients undergoing cardiac surgery and compared one group provided with information 5 - 14 days prior to admission (experimental group) with a control group who received routine teaching on admission. In the postoperative period the experimental group had a greater level of knowledge, more positive self-reported mood states and increased physiological recovery. However, no differences were observed in anxiety levels even though, in comparison, the experimental group spent a far greater amount of time with the nursing staff.

Oberle et al (1994) surveyed 294 patients undergoing various surgical procedures. A large number of patients were dissatisfied with the timing of information provision as the bulk of it occurred on the ward immediately prior to surgery. It was concluded the majority of patients would have preferred to receive information prior to admission. Scriven and Tucker (1997) evaluated 184 leaflets collected from 97 hospitals, concerning one particular surgical technique - hysterectomy. Only 35\% of the hospitals gave their available hospital leaflets at the optimum time i.e. prior to admission. Mitchell (1997) surveyed 150 patients undergoing minor gynaecological day surgery and general anaesthesia. Six percent stated they would have preferred to receive 
the information a few months prior to surgery, $24 \%$ a few weeks prior to surgery, $48 \%$ a few days before surgery and $20 \%$ a few hours before surgery.

\section{Indicators Against Information Provision}

Finally, for many years the utility of information provision has been debated in a number of studies and frequently determined not to be required. In a study by Christopherson and Pfeiffer (1980) 41 patients were asked to read an information booklet prior to cardiac surgery. As many patients completely refused to read any pre-surgery material, the control group was self-selecting. Ziemer (1983) provided differing audiotaped information to 111 patients on the eve of surgery. The participants were divided into three groups i.e. procedural information only, procedural and sensory information, sensory and coping information. No significant differences utilising physiological and emotional measures were established i.e. the provision of extra information was not observed to be beneficial.

Goldmann et al (1988) studied 52 female patients undergoing general anaesthesia for a gynaecological procedure. Participants were divided into two groups prior to anaesthesia where one group received extra information and the second group received three minutes of hypnosis. The main conclusions were associated with the information provision. "The provision of information does not have a uniformly positive effect. Patients may either wish to be informed about the details of their operation, remain uninformed, or a mixture of both." (p. 468). Lepczyk et al (1990) studied 72 patients following open-heart surgery and discovered $81 \%$ of patients once told of their need for surgery sought out details 
regarding their operation themselves. Therefore, they may not have required any additional information once admitted to hospital. Adequate information may have already been gained via the media, general practitioner, friends and relatives (Donovan and Blake 1992, Avis 1994). Elsass et al (1987b) studied 74 patients admitted for elective general surgery and $84 \%$ stated the emotional support provided by the nurses pre-operatively was more effective than the written information provided. Moreover, detailed medical information provided by the anaesthetist only served to increase anxiety for some patients. Martelli et al (1987) studied 46 patients undergoing oral surgery and local anaesthesia and concluded emotional focused coping strategies was the most effective method for patients who were very anxious and in a situation where there was little possibility for personal choice. Anderson (1987), utilising emotional and behavioural measures when surveying patients prior to cardiac surgery concluded information alone did not reduce anxiety although it did help to increase feelings of control.

Salmon et al (1986) and Salmon (1992a) following a study of 17 patients undergoing surgery and general anaesthesia suggested forced compliance with a pre-operative educational programme actually led to an increase in anxiety. Significantly higher levels of cortisol were excreted in the urine of patients on a ward where they were requested to comply with pre-operative procedural information giving and reassurance. Kerrigan et al (1993) surveyed 96 men undergoing elective inguinal hernia repair under general anaesthesia. The aim was to observe the possible changes in anxiety after receiving detailed 
information concerning potential complications. While the detailed information did not increase patients' self-ratings of anxiety, $25 \%$ of those who randomly received the detailed information stated they had received too much. Hawkshaw (1994) conducted a telephone follow-up call to 1,008 patients the morning after day surgery. Patients were seen to require differing levels of information concerning their surgical experience. "This is reflected in the 729 (72.3\%) patients who reported that they were happy with the information they had acquired. This includes $274(27.2 \%)$ who received no information but were satisfied." (p. 349).

Lamarche et al (1998), in a quasi-experimental study divided 54 patients into two groups. Twenty-eight patients in the experimental group were telephoned prior to cardiac surgery in order to provide information and the opportunity to pose questions. Twenty-six participants in the control group received no telephone call - merely routine care. Utilising a visual analogue scale on the day of admission in order to measure anxiety, a significantly higher level of anxiety was established in the experimental group i.e. the extra information had increased anxiety. Stanley et al (1998) utilising emotional measures on two separate occasions i.e. prior to surgery and six weeks after discharge, were unable to establish a difference in anxiety or knowledge level between a routine consent group and a routine consent plus extra information group. Bondy et al (1999) in a quasi-experimental research study sent information pamphlets and a 10-minute videotaped presentation to each patient in the experimental group while the control group merely received routine care i.e. no additional written 
information. Utilising a self-administered anxiety questionnaire (Spielberger et al 1983) the experimental group were significantly less anxious immediately prior to surgery. However, of the 65 participants in the experimental group, $10 \%$ preferred no information and in the control group 24\% preferred (and received) no information. An element of self-selection by the control group may have occurred.

Teasdale (1993) in a critical appraisal of the relationship between information provision and anxiety reduction emphasised that to assume one automatically follows the other may be an oversimplification of a very complex issue i.e.

\section{PATIENT + INFORMATION = REDUCED ANXIETY}

He suggests all information is partial as it is impossible to tell the patient everything and extremely difficult to be truly objective. Additionally, it is very difficult to remain truly confident the information is required or indeed that it has been properly understood. Unless requested, it is unclear if the patient wishes to receive the information being provided. Moreover, factual information given to the patient, however neutral and objective, may not be interpreted in the way in which it was originally intended. "Therefore, to ask whether 'information' relieves anxiety is conceptually flawed." (Teasdale 1993 p. 1128). It can only be assumed when using this mechanistic model, the information provided has had the desired effect i.e. reduced the patients' anxiety or improved their ability to cope. Such answers are very difficult to establish (Fareed 1994). This approach is reflected in the psychological theory of vigilant and avoidant coping i.e. some 
people may not benefit from the acquisition of information in the pre-operative phase.

Problems also exist in the balance between doctor/ patient interactions as Guadagnoli and Ward (1998) state "...there will always be some imbalance in the patient-physician relationship since the patient is sick and vulnerable and the physician has the expert knowledge." (p. 336). Patients in this situation may feel it unwise, even foolish (rightly or wrongly) to question the 'specialist' i.e. they trust the doctor and may not wish to challenge his/ her wisdom. In addition, the standard of written information patients receive is problematic. The readability of information regarding surgical consent and the amount of information provided were identified by Kent (1996) as two central problematic issues. Moreover, Scriven and Tucker (1997) when examining 184 educational leaflets from 97 hospitals in England point to a lack of evaluation of the leaflets' effectiveness and the assumption the information was of value to patients. Much information was assumed to be required although not proven to be. With little external consultation, hospital staff frequently produce leaflets covering information 'they' deem necessary.

In a literature review on patient education by Webber (1990) the goal of all models of patient educational material examined was to gain compliance with the health professional's advice. Education was therefore seen as a means of controlling the situation and gaining an obedient, compliant patient as opposed to empowering the patient and providing choice. "Patients remain dependent upon their healthcare provider and are expected to be both obedient and 
grateful." (Webber 1990 p. 1091). In a more recent review of patient information leaflets Dixon-Woods (2001) supports this view. It was stated that the majority of patient information leaflets reviewed had a bio-medical agenda. Information under this model was provided for three reasons - medico-legal protection, gaining patient compliance and paternalism i.e. dealing with irrational, passive, forgetful incompetent patients. Entwistle et al (1997) also alluded to this by questioning the motives of providing patients with information, as there could be a hidden agenda. "For example, a distinction may be made between those initiatives which aim particularly to enhance patient autonomy by helping people to make their own free choices and those which aim to persuade people to accept a particular choice (typically the one deemed most clinically - and/ or cost-effective by the initiative's promoters." (Entwistle et al 1997 p. 69). This is in direct opposition to a fundamental approach within nursing care as highlighted by Malin and Teasdale (1991) in a review of the literature on patient empowerment. "Empowerment implies that nurses must maximise patients' independence and minimise their dependence." (р. 658). In order to minimise dependence, patients must firstly have the information they require. This may necessitate less emphasis being placed upon the information hospital personnel consider patients require and more upon what patients consider they require.

In summary, over many decades the differing categories of information have all demonstrated positive results although the main approaches appear consistently to be procedural, behavioural and sensory information. The most effective form of information provision is a perplexing issue as some aspects of 
information provision can be viewed as assisting problem focused coping with surgery while other information assisting more the emotional focused aspects of coping. This has led to the term psycho-educational information provision being adopted by numerous studies. Problem focused coping information provision is viewed as the main form of pre-operative information although no distinction between problem focused and emotional focused coping exists in practice. Additionally, little or no choice is provided regarding the type of information received.

A number of studies have provided indicators for the provision of information. Adequate information provision has been observed to reduce stress merely as a result of the person being better informed. The mode of information delivery is also a complex issue as some individuals prefer written information, verbal information, a mixture of the two or written information plus a videotaped presentation. Additionally, the timing of information provision is problematic. When pre-operative anxiety begins and when it diminishes has yet to be clearly established. The majority of patients undoubtedly require some information prior to hospital admission. Some prefer this to be a few days or weeks before, while others do not require any until the day of admission.

Conversely, not all patients desire to be fully informed. It is very difficult to gauge the information requirements of each patient, or indeed, if the information provided has had the desired effect. Additionally, information provision may not automatically lead to a reduction in anxiety for all patients. Rogers and Reich (1986), in a comprehensive review of the literature dating back to 1950, 
concluded all aspects of information provision have worked at some stage i.e. all forms of information provision, relaxation, specialist support, modelling, hypnosis and close support from the nurse. Therefore, Rogers and Reich (1986) maintain the answer must lie with the individual's preferred method of preparation.

The need for information has increased considerably (Chapter 4) with the rise in day surgery activity as the opportunity and time in which to gain information has been substantially reduced. Moreover, the contentious issues surrounding information provision remain problematic i.e. who requires information, when do they require it and how much do they require? Asking patients prior to day surgery what level of information they would prefer and then providing this preferred level prior to admission, at an agreed time, may greatly enhance their ability to cope with surgery i.e. providing a detailed level of information to patients with a vigilant coping style 1 to 2 weeks prior to their surgery and a simple level of information to patients with an avoidant coping style approximately 5 to 7 days before surgery. This is the way in which this study will progress i.e. detailed and simple information sent out at random to day surgery patients 7 to 10 days prior to surgery. However, what must firstly be established is that information provision within modern adult elective day surgery is indeed a challenging issue. Studies which draw conclusions concerning information provision from data obtained from in-patient studies carry little validity within modern surgery, for the reasons discussed in Chapter 1 i.e. patient preoperative self-preparation, nominal nurse/ patient contact, minimal access 
surgery, rapid anaesthesia, rapid post-operative recovery, 61/2 hours average length of hospital stay and post-operative recovery at home. Therefore, a review of the literature, which expressly examines the views of day surgery patients, is required in order to confirm information provision as a central challenge for day case surgery. 


\section{ChAPTER 4}

\section{Patients' Perceptions of Day Surgery}

\section{Literature Review}

Within this chapter a review of the literature concerning studies that have exclusively sought the views and experiences of day surgery patients will be considered. A review of the literature examining the views of patients undergoing day surgery was required, as no other review has focused solely upon day surgery. The literature review employed Medline (medical database), CINAHL (Cumulative Index to Nursing and Allied Health Literature), a crossreference search and a hand search. The key word combinations utilised were day surgery and satisfaction, ambulatory surgery and satisfaction, anxiety and day surgery, and anxiety and ambulatory surgery.

The earliest studies were from 1978 and 1979. Seventeen studies emanated from the 1980's, eighty-eight from the 1990's (80\% of the total), two from 2000 and one from 2001 . The figure of $80 \%$ in the 1990's clearly demonstrates the intense interest and subsequent rapid rise in day surgery activity in comparison with previous decades. The majority of the studies were undertaken in Europe, America and Australia (Figure 1 p.98). A total of 110 studies were identified both large and small, all of which were utilised, as at present, the number of full research studies within day surgery is limited. Furthermore, a systematic review of the literature expressly concerning patients' experiences of day surgery has not been previously undertaken. 


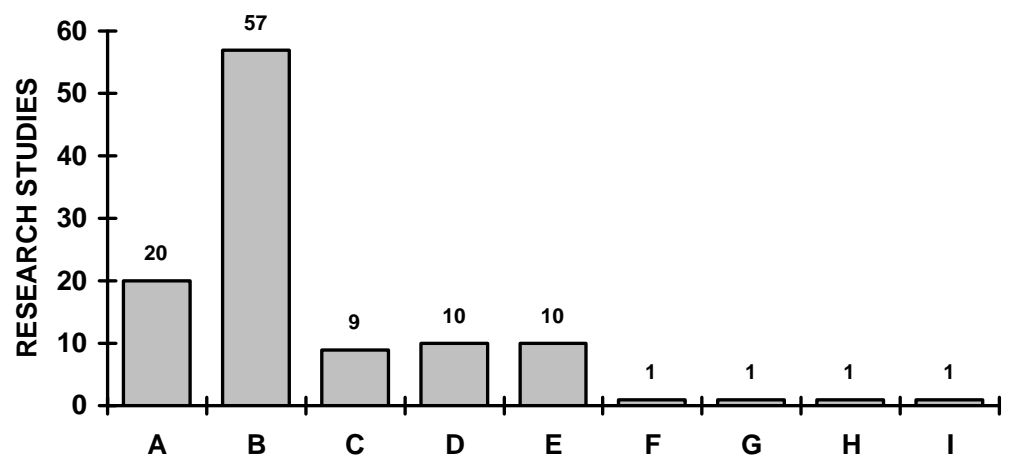

$$
\begin{aligned}
& \text { A) UNITED STATES of AMERICA } \\
& \text { B) EUROPE } \\
& \text { C) AUSTRALIA } \\
& \text { D) EUROPE (Mainland) } \\
& \text { E) CANADA } \\
& \text { F) AFRICA (Ghana) } \\
& \text { G) ISRAEL } \\
& \text { H) ICELAND } \\
& \text { I) NEW ZEALAND }
\end{aligned}
$$

Studies, which merely asked "Were you satisfied with day surgery?" (of which some did) were obviously far too brief and therefore omitted. The only criteria for inclusion was that data be collected specifically from elective, adult day surgery patients undergoing general, local or regional anaesthesia for general surgery (e.g. no ophthalmic surgery) and be expressly concerned with the patients' self-reported experiences (an overview of all the studies is provided in table format within the respective section).

For clarity of explanation each study will be classified under the heading of audit (descriptive statistics only), qualitative research (phenomenological) and quantitative research (survey and quasi-experiment) (Table 2 p.99). In this way the depth of analysis within each study can be briefly determined. Four main themes emerged from the review and relate to nursing and anaesthetic practice, information provision, the patients' experience of day surgery and recovery at home. These themes will therefore be explored in greater depth. 
TABLE 2

CLASSIFICATION OF ALL STUDIES REVIEWED CONCERNING PATIENTS' PERCEPTIONS OF DAY SURGERY

\begin{tabular}{|c|c|c|c|}
\hline & & \multicolumn{2}{|l|}{ QUANTITATIVE } \\
\hline & & SURVEY & QUASI-EXPERIMENTAL \\
\hline $\begin{array}{l}995) \\
.997) \\
\text { ffart }\end{array}$ & $\begin{array}{l}\text { ) } \\
\text { m } \\
\text { s } \\
\text { ) } \\
\text { (7) } \\
\text { (9) } \\
\text { (9) }\end{array}$ & $\begin{array}{l}\text { Agboola et al (1998) } \\
\text { Bhattacharya et al (1998) } \\
\text { Birch et al (1993) } \\
\text { Brumfield et al (1996) } \\
\text { Buttery et al (1993) } \\
\text { Caldwell (1991) } \\
\text { Callesen et al (1998) } \\
\text { Cozzarelli (1993) } \\
\text { Daoud \& Hasan (1999) } \\
\text { Donoghue et al (1998) } \\
\text { Fraser et al (1989) } \\
\text { Frisch et al (1990) } \\
\text { Fung and Cohen 2001 } \\
\text { Gamotis et al (1988) } \\
\text { Ghosh \& Sallam (1994) } \\
\text { Gnanalingham \& Budhoo } \\
\text { (1998) } \\
\text { Guilbert \& Roter (1997) } \\
\text { Haldane et al (1998) } \\
\text { Harju (1991) } \\
\text { Icenhour (1988) } \\
\text { Jamison et al (1987) } \\
\text { Jennings \& Sherman } \\
\text { (1987) } \\
\text { Kain et al (1997) } \\
\text { Lawrence et al (1997) } \\
\text { Limb et al (2000) } \\
\text { Lindén \& Engberg (1995) } \\
\text { Lindén \& Engberg (1996) } \\
\text { Lisko (1995) } \\
\text { Mackenzie (1989) } \\
\text { Male (1981) } \\
\text { Michaels et al (1992) } \\
\text { Mitchell (1997) } \\
\text { Nkyekyer (1996) } \\
\text { Nyamathi \& Kashiwabara } \\
\text { (1988) } \\
\text { Oberle et al (1994) } \\
\text { O'Connor et al (1991) } \\
\text { Parsons et al (1993) } \\
\text { Philip (1992) } \\
\text { Pineault et al (1985) } \\
\text { Pollock \& Trendholm } \\
\text { (1997) } \\
\text { Ratcliffe et al (1994) } \\
\text { Read (1990) } \\
\text { RCS and East Anglia } \\
\text { R.H.A. (1995) } \\
\text { Sigurdardottir (1996) } \\
\text { Singleton et al (1996) } \\
\text { Smith (1998) } \\
\text { Wallace (1986c) } \\
\text { Wedderburn et al (1996) } \\
\text { Willis et al (1997) } \\
\text { Winwood \& Jago (1993) }\end{array}$ & $\begin{array}{l}\text { Aasboe, et al (1998) } \\
\text { Alkaissi et al (1999) } \\
\text { Augustin \& Hains (1996) } \\
\text { Claxton et al (1997) } \\
\text { Coslow \& Eddy (1998) } \\
\text { Cundy \& Read (1981) } \\
\text { Domar et al (1987) } \\
\text { Done \& Lee (1998) } \\
\text { Edwards et al (1991) } \\
\text { Gaberson (1995) } \\
\text { Goldmann et al (1988) } \\
\text { Hulme et al (1999) } \\
\text { Kempe \& Gelazis (1985) } \\
\text { Markland \& Hardy (1993) } \\
\text { Martikainen et al (2000) } \\
\text { Mealy et al (1996) } \\
\text { Parlow et al (1999) } \\
\text { Towey et al (1979) } \\
\text { Vogelsang (1990) } \\
\text { Wicklin \& Forster (1994) } \\
\text { Zvara et al (1996) }\end{array}$ \\
\hline 6 & & 50 & 21 \\
\hline
\end{tabular}




\section{Nursing and Anaesthetic Practice (Table 3 p.100a).}

The issues to be discussed here are attendance to a pre-assessment clinic, anxiety management and home preparation. Information provision was implicit within all of these themes although because of the magnitude of information provision it will be considered separately.

Firstly, the need for a pre-assessment check was a strong theme in order to i) help adequately assess the patient's fitness for surgery, ii) provide instructions regarding care and recovery at home and, iii) increase nurse/ patient contact time in order to help allay fears. An audit by Bottrill (1994) revealed preassessment clinics to be beneficial as nurses had more time to explain treatment with patients. This in turn led to a reduction in the junior doctors' workload as it reduced some of the responsibilities of explaining care and treatment. However, this audit only gained a $37 \%$ response rate and focused upon gynaecological patients only. In a large survey by Guilbert and Roter (1997) concerning coping at home after surgery the most important determinant of satisfaction was patient preparation i.e. effective communication and instruction. In a study specifically concerning teaching in day surgery, Brumfield et al (1996) interviewed both patients and nurses regarding information provision. Patients preferred teaching to take place prior to admission. This conflicted with the nurses' views as they thought it should take place on the day of admission. In a similar study employing patients undergoing laparoscopic surgery, Wallace (1986c) compared in-patient and day-case patient outcomes. Day-case patients were more anxious on the morning of surgery and $47 \%$ had 
to remain in hospital overnight following surgery. It is suggested this increase in overnight stay may have been due, in part, to a lack of adequate preparation. However, participants were given no choice as to whether they underwent day surgery or in-patient surgery.

In a survey by Harju (1991) data were collected using a small questionnaire three months after surgery. This revealed $74 \%$ of patients to be satisfied with day surgery. This was attributed, in part, to good patient selection and adequate pre-operative teaching within the pre-assessment clinic. Kleinbeck and Hoffart (1994) interviewed 19 patients by telephone for approximately 20 minutes on two occasions in the post-operative period. Participants felt quite vulnerable when going home and were unsure about what activities they could perform around the house as clear instructions had not been received pre-operatively. This led to trial and error learning of everyday activities around the home. An audit by Rudkin et al (1996) also highlighted the strengths and weaknesses of day surgery pre-assessment clinics. Dedicated day surgery facilities i.e. designated ward and theatre used only for day case patients, and mixed day surgery facilities i.e. no separate ward or theatre, were compared in 8 Australasian day surgery centres. It was concluded information provision, waiting time and general satisfaction within dedicated day surgery units was superior to mixed in-patient facilities.

Pre-assessment clinics were therefore broadly observed in many studies to be a very important way of providing patients with essential information i.e. fasting times, pain management, arrival and departure times. Also, dedicated 
day surgery units demonstrated greater efficiency when dealing with day case patients.

The second aspect within this section concerns anxiety management. In an audit, which examined satisfaction with anaesthesia, Gupta et al (1994) discovered $50 \%$ of patients would have preferred a pre-medication to help allay fears. It was therefore recommended greater emphasis should be placed upon interventions designed to help reduce anxiety in the pre-operative stages. Moreover, the study recommended anxiety reduction techniques may be necessary irrespective of the type of anaesthetic used i.e. local, general or regional. Following a study employing a quasi-experimental design, Mealy et al (1996) recommended the use of a beta-blocker (Propranolol 10 mgs to reduce the heart rate) to be routinely given to all patients to help combat the physiological effects of anxiety. A further three studies also suggested the use of pre-medication to help allay fears and relieve anxiety (Male 1981, Gupta et al 1994, Mackenzie 1989).

Winwood and Jago (1993) recommended the use of Diprivan (Propofol) as the drug of choice for induction of general anaesthesia. Diprivan produced a less anxious patient in the post-operative phase in comparison to a more traditional anaesthetic agent (Thiopentone). However, both groups in the survey were given drugs with no comparison group i.e. improved information group, usual care group. Novice patients, patients with previous unpleasant experiences, female patients and patients undergoing oral surgery all had increased anxiety in a survey by Mackenzie (1989). It was recommended that 
once identified, anxious patients could be given reassurance and anxiolytic medication (anxiety reducing drug). A number of in-patient and day case patient studies have suggested general anaesthesia causes considerable anxiety for patients whereas others have suggested patients undergoing local anaesthesia to be more anxious or more dissatisfied (Ramsay 1972, Ryan 1975, Male 1981, McCleane and Cooper 1990, Mitchell 1997, McGaw and Hanna 1998, Birch et al 1993, Chung et al 1994).

In a study employing a quasi-experimental design by Domar et al (1987) the benefits of patient contact prior to surgery for instruction purposes were highlighted. Patients on a programme of relaxation reported being less anxious in the days prior to surgery. The experimental group however, received far greater attention than the control group, which could have influenced the data i.e. the experimental group were telephoned each week to ensure compliance with relaxation therapy. Moreover, the operations performed were not simple as all patients involved were undergoing surgery for skin cancer. In a further quasiexperimental design, Markland and Hardy (1993) obtained positive results when also investigating relaxation prior to surgery. However, the experimental group received 22 minutes of relaxation immediately prior to surgery whereas the control group only received 'routine ward care'. Gaberson (1995), in an effort to help reduce anxiety, examined the possible benefits of distraction as part of the pre-operative preparation process. Distraction via audiotape was carried out once following admission and was 20 minutes in duration. No significant reduction in anxiety was established although participants underwent a variety 
of surgical procedures employing both local and general anaesthesia. Such a range of surgical procedures and methods of anaesthesia could have influenced the results. Augustin and Hains (1996) also studied patients about to undergo differing surgical procedures and both local and general anaesthesia. A reduction in anxiety was achieved when participants were allowed to listen to a choice of audiotaped music. However, a significant difference only occurred on one physiological measure.

In a survey by Gamotis et al (1988) in which in-patient and day-patient satisfaction were compared, day-patients were more satisfied. This was due, in part, to their interaction with staff as it was more structured i.e. in the preassessment clinic and on the day of surgery. However, the female in-patients were more satisfied with the relationship they had established with the nurses. This may indicate the comparatively shorter time spent in day surgery did not allow sufficient time for a therapeutic relationship to develop via the use of the nurses' interpersonal skills. The physical presence of the nurse has been observed to have a positive effect upon anxiety management. Parsons et al (1993) conducted a small survey to ascertain which behaviours were deemed to be the most caring behaviours by patients. Various categories emerged but the three most effective caring behaviours identified were the nurses' reassuring presence, verbal reassurance and attention to physical comfort. Therefore, the nurse just being in close proximity to the patient while he/ she was in the day surgery facility and expressing concern was viewed as very helpful during periods of increased anxiety. Cozzarelli (1993) in a comprehensive study 
surveyed 112 female patients undergoing day surgery for termination of pregnancy. This type of surgery may have carried a higher emotive value and may have contributed, in part, for the reduced number of participants (38\%) who completed all three stages of the study. However, it was concluded that enhancing self-efficacy (perceived ability to perform a task well or cope well with a procedure) by providing positive feedback was a vital therapeutic aspect of the nurses' role.

In a quasi-experimental design by Vogelsang (1990) the importance of the therapeutic role of the nurse was further highlighted. It was established that continued contact with one nurse i.e. from the pre-assessment clinic through to discharge on the day of surgery, improved satisfaction with care and led to earlier discharge. However, the highly individual contact in the experimental group, which also encompassed 60 to 88 minutes contact in the post-operative period, was compared to routine care only. Icenhour (1988) established that some patients felt rushed and unable to retain all the relevant instructions and necessary information during routine admission. Post-operative instructions and information were retained and adhered to more when a relative was present immediately prior to discharge i.e. the therapeutic role can also be undertaken by a relative.

Numerous studies therefore indicate effective anxiety management to be influential for the elective adult day surgery patient. The continued presence of the nurse, although highly recommended, may be very limited during a modern day surgery operating schedule i.e. rapid throughput of patients. It is evident 
throughout the review, spanning 3 decades that little or no formal aspects of psycho-educational care, although recommended, have been implemented. The use of pre-medication appears to be the only consistent exception. Drugs to reduce anxiety have been utilised for the very anxious patient although such intervention is limited. Pharmacological intervention for anxiety assumes that i) anxiety is only a problem for the overtly anxious patient as it may hinder induction of anaesthesia, ii) anxiety only begins once admitted to hospital, and iii) anxiety ceases immediately following surgery.

The final aspect in this section concerns preparation for discharge home. Discussions regarding recovery from surgery at home were viewed as an essential part of the pre-assessment visit (see 'Recovery at Home'). Pain was identified as a considerable problem following day surgery in an audit by Firth (1991). Patients had expected to have some pain but had not purchased any analgesia prior to admission. This was either because they had expected the hospital to provide analgesia or they had not been adequately instructed prior to admission. To improve the problem of post-operative pain management it was suggested in audits by Lewin and Razis (1995) and Marquardt and Razis (1996) that pre-packed analgesia or analgesia packs with the relevant accompanying information be provided. These packs could vary according to the operation type and help to establish a more effective programme of pain management. However, this may involve the nursing staff establishing which pack to administer, explaining the accompanying information and possibly, in some instances, securing payment. Thatcher (1996) in a qualitative study interviewed 
four patients 2 to 4 days following surgery. It was established that patients expected to experience some pain on discharge but when the recommended or prescribed analgesia did not bring relief, they found it difficult to cope. One patient was required to pay for the prescribed analgesia while in hospital and subsequently refused the medication. Sufficient information providing a clear and realistic image of post-operative recovery may help prevent such occurrences.

Discharge from a day surgery facility is largely based on medical criteria i.e. conscious with a lack of post-operative morbidity (medical complications in the post-operative period). Stephenson (1990) employing a small audit established some discharge guidelines. It was discovered no patient was fully alert after 30 minutes and $57 \%$ experienced drowsiness within the first 24 hours. This indicated less than half of patients reached an adequate level of consciousness at the time of their discharge. Eight studies (see 'Recovery at Home') recommend a telephone helpline be established following discharge to routinely contact patients within the immediate post-operative period in order to check progress. In a telephone follow-up survey conducted by Hawkshaw (1994) to evaluate satisfaction with care, one of the unforeseen benefits resulting from this form of data collection was that patients viewed the telephone interview as a valuable part of their care and a chance to ask questions.

Patients were therefore clearly in need of enhanced information provision following discharge as much recovery was occurring at home. Frequently, unforeseen events caused many problems for patients struggling to regain full 
fitness as a result of the limited information available i.e. how best to proceed in the event of an unforeseen occurrence. The opportunity to speak with a healthcare professional from the hospital or day surgery unit was of considerable benefit.

Information Provision (Table 4 p.108a).

The largest theme highlighted by 60 studies (54.5\% of total) and implicit in many others relates to informational provision. More specifically the amount of information provided, the differing levels of information required, the mode of provision and timing of provision. Firstly, the issue of receiving the correct amount of information will be considered.

In a comprehensive study by Pollock and Trendholm (1997) for 'Which' independent consumer guide magazine (such is the public interest in day surgery), information provision was revealed to be a major issue. "It was clear from our survey that people who were given the least information were the most dissatisfied with day surgery." (Pollock and Trendholm 1997 p. 16). The Royal College of Surgeons of England and East Anglia Regional Health Authority (1995) conducted a comprehensive and highly informative survey in which data were collected both on a regional and local basis. The survey incorporated 10 day surgery units, 30 consultant surgeons and 1,434 patients. Patients expressed many concerns regarding their forthcoming surgery although information provision was of most concern. The study went on to say that although $75 \%$ of the patients were satisfied with the care and information they 
had received "This overall appraisal conceals significant levels of dissatisfaction in certain areas...." (p. 2).

In a qualitative study by Otte (1996) eight patients were interviewed three weeks after Ear, Nose and Throat surgery. Participants preferred the convenience of day surgery, as it was less disruptive to their lifestyle although they experienced major problems with communication. All participants stated they were unprepared for their surgery in terms of information received and the educational support provided. However, all patients underwent surgery in a mixed day surgery facility. Mixed facilities have been viewed as far less efficient than dedicated facilities (Rudkin et al 1996). In a quasi-experimental research design by Kempe and Gelazis (1985) the effects of pre-operative written and verbal information on anxiety were studied. It was concluded that greater psychological preparation i.e. an increased amount of information, resulted in a less anxious patient. However, no account was given to the effect of the considerable increase in attention provided to the participants in the experimental group or that they were given a systematic programme of information.

Nkyekyer (1996) undertook a survey to explore participants' experiences of laparoscopic surgery. Twenty-nine percent of participants were unhappy with the level of information they had received. However, this figure may be misleading and could be even higher as data were collected two weeks after surgery in the hospital out-patient department. Asking patients to comment about their care whilst still undergoing medical treatment has been observed to 
give rise to inaccurate responses (Fitzpatrick and Hopkins 1983). Menon (1998) surveyed 78 patients prior to surgery and this phenomenon was again demonstrated. It was revealed $66 \%$ of participants would have preferred more information and yet they stated the quality of the service to be good. However, all participants had undergone vasectomy, which may carry a higher emotive value and consequently a greater degree of information may have been required. In an attempt to circumvent the possible issue of under-reporting whilst still undergoing medical treatment, a number of studies have utilised postal questionnaires following discharge.

Sigurdardottir (1996) in a postal survey compared satisfaction with care between two day surgery facilities. The main areas of concern stemmed from the lack of adequate information as, "The patients were least satisfied with items related to the educational sub-scale (patient's knowledge regarding their operation and treatment) as they seldom received any booklets or pamphlets relating to the surgery." (p. 73). Again, however, one of the day surgery facilities within this study was a mixed facility i.e. not a dedicated day surgery unit. In a survey by Buttery et al (1993), although only using a short postal questionnaire, it was established most patients were satisfied with day surgery. However, the main criticisms centred on the long pre-operative waiting period, lack of postoperative privacy and the provision of insufficient information. Willis et al (1997) in a postal survey discovered positive correlations between receiving written information and level of satisfaction, and receiving an explanation and recommending day surgery to a friend. 
An audit by MacAndie and Bingham (1998) examined patient satisfaction and general practitioner involvement in care. Twenty percent stated their discharge information was excellent and 50\% good, while the remainder were dissatisfied. King (1989) revealed in an audit, from the participants who returned the questionnaire (44\% response rate), $30 \%$ received no written information concerning their care although $97 \%$ were happy with their discharge information. A 44\% response rate, although good for a postal questionnaire, may hide the true level of satisfaction. Are those who did not reply, too satisfied or too dissatisfied to do so? To avoid such postal problems, Fitzpatrick et al (1998) utilised a small telephone survey and established the need for specific information in the post-operative period. However, this survey, as with a considerable number of other medical surveys, maintained a heavy postoperative morbidity focus.

Fung and Cohen (2001) surveyed 30 patients undergoing surgery and general anaesthesia regarding aspects of care. Fifteen senior anaesthetists were also surveyed utilising the same questionnaire in order to check for parity. Both participants and anaesthetists were requested to rank in order of importance the care they valued the most. In each phase of their care i.e. preoperative, intra-operative, pre-discharge and post-discharge, participants ranked information provision and communication as the most important. The anaesthetist's, although able to state what patients required on discharge, were unable to predict what was required the most both in the pre-operative phase and immediately prior to surgery. 
Information provision for day surgery patients is therefore undoubtedly a considerable issue. The studies referred to above have dealt with information as a central issue. However, many studies within this review also cite information provision as a challenging issue although have not studied information provision directly. Additionally, information provision presents greater difficulties in hospitals that do not have a dedicated day surgery unit i.e. ward and theatre used only for day surgery.

The second aspect of information provision concerns the level of information required. Eight studies have highlighted an inherent dilemma with information provision in that too much and also too little can cause an increase in anxiety. Following a telephone survey of almost 300 patients by Oberle et al (1994) it was stated -

"Although 25\% of patients indicated that they had received little or no information about their surgery and post-operative course, some of them were satisfied with that; they indicated that they simply preferred not to have any details about their upcoming surgery, because the more they knew, the more frightened they would become." (p. 1024).

Kain et al (1997) studied 197 patients undergoing a variety of surgical procedures and general anaesthesia. The aim of the study was to determine what information patients required from their anaesthetist in the pre-operative period and to identify which patients desired more information. Implicit in this study therefore was the general assumption that some patients required more information than others. This American study concluded a generally higher level 
of information was desired by female patients and divorced patients. However, it has been suggested by Lonsdale and Hutchison (1991) that the desire for information may be influenced by cultural i.e. patients in American may require and expect a far greater level of information.

In a quasi-experimental design by Goldmann et al (1988) the effects of hypnosis and information provision on anxiety were examined. Immediately prior to surgery participants received an 8-minute structured interview regarding knowledge of surgery followed by either a neutral discussion regarding social aspects of surgery $(n=27)$, or 3 minutes of hypnosis $(n=25)$. A mean, significant difference in anxiety scores was achieved for patients who had undergone 3 minutes of hypnosis prior to surgery. This may indicate that for some patients, anxiety may not be aided by information provision alone. Additionally, it was established that not all patients desired the same amount of information - some required more others less. The question most frequently asked of the anaesthetist was whether induction of anaesthesia would be by mask or needle and the most informative aspect for the majority of patients was to be told of the length of anaesthesia (Goldmann et al 1988). In a survey by Mitchell (1997) female patients scheduled for day surgery were interviewed immediately prior to surgery and general anaesthesia. The aim of the study was to establish a possible link between individual information requirements and health locus of control. No link was established although again the need for differing levels of information was recognised. Forty-one percent of participants would have preferred a detailed information booklet and 53\% a simple 
information booklet. However, the data were collected from gynaecological patients only and at a very anxious period i.e. approximately 1 to $1 \frac{1 / 2}{2}$ hours prior to surgery.

Lindén and Engberg $(1995,1996)$ utilised 105 patients in a satisfaction with care survey, three weeks after surgery. Thirty-six percent thought the information provided was insufficient and a reduced level of information was positively associated with an increase in post-operative morbidity. A patient satisfaction survey by De Jesus et al (1996) revealed dissatisfaction with information because of its lack of adaptation to home recovery. Again, it was established that not all patients required the same level of information. Donoghue et al (1998) concluded patients preferred day surgery as it caused minimum disruption to their lifestyle although "Men who received information about the day surgery procedure were less anxious than their peers who said that they had received insufficient information." (p. 195). However, all participants underwent a cystoscopy (fine tube passed via the urethra to view prostate and bladder), which again may carry a higher emotive value. An extensive survey by Caldwell (1991) concluded patients might have different information requirements as those who had a greater need for information and received extra information, experienced less pre-operative anxiety. However, $43 \%$ of the patients within this study were uncertain about a diagnosis of malignancy, which may have strongly influenced the desire for information.

The desire for day surgery patients to receive more information is clear. However, what is also apparent is that this does not apply to all patients. Some 
patients are satisfied with the amount of information they receive whereas others are not. While numerous studies may have identified the patients who require more information, a systematic plan to deliver the additional information to the patients who desire it has not, as yet, been undertaken.

The third aspect of information provision concerns the mode of provision. Five studies have demonstrated the need for patients not only to receive verbal and written information but also have the chance to view/ hear, or take home to view/ hear, a videotaped/ audiotaped presentation concerning the surgical procedure (Baskerville et al 1985, Wicklin and Forster 1994, Lisko 1995, Zvara et al 1996, Done and Lee 1998). A study by Wicklin and Forster (1994) employing a quasi-experimental design was conducted to establish whether the modelling of behaviours from a videotaped presentation was of greater benefit to patients than the provision of written information. The only conclusion from the study however, was that females reported a greater level of pre-operative anxiety than males. The number of times the presentation was viewed and the duration of the presentation were not detailed although such factors could have influenced the outcomes.

Lisko (1995) conducted a small pilot survey in which gynaecological patients viewed a short videotaped presentation. The purpose was to encourage greater autonomy although no significant results were established. This may, in part, have been due to the presentation being only 8 minutes in duration. Done and Lee (1998) demonstrated that the knowledge level of patients could be increased if they were able, on the day of surgery, to view a short videotaped 
presentation concerning anaesthesia. Knowledge scores were indeed better in the video group in comparison to the control group although no difference in anxiety was established. In a quasi-experimental study by Zvara et al (1996) participants were divided into two groups, 2 weeks prior to surgery. One group received a pre-operative videotaped presentation and the other group, no video. Using a post-video test only, knowledge in one area was deemed to have been significantly improved i.e. what to do if feeling ill on the proposed day of surgery. In an audit by Baskerville et al (1985) over a nine-month trial period, participants were provided with an audiotaped presentation concerning their operation. The information was well received and highlighted the need for information prior to the day of surgery. Finally, Mitchell (1997) surveyed 150 day surgery patients undergoing general anaesthesia for gynaecological surgery and established $83 \%$ would have preferred to view a videotaped presentation concerning their surgical experience.

Day surgery patients may prefer information in the form of videotaped or audiotaped presentations as such material can be viewed and discussed at home with their potential carer. The need to share information in this way may be considered logical given the responsibility of care placed upon the relative/ friend in the post-operative period. Additionally, a good deal of health education material is currently provided in this way and thereby people may understandably expect to have the choice of differing modes of provision. However, the vast majority of patients required information in written format. Written information may therefore be viewed as the preferred primary source of 
information with videotaped and audiotaped information as a highly desirable but secondary source.

The final aspect of information provision concerns the timing of provision and discharge provision (Oberle et al 1994, Brumfield et al 1996, Mitchell 1997). In a survey by Brumfield et al (1996) it was established that patients preferred teaching to take place prior to admission. Mitchell (1997) revealed 48\% of patients surveyed would have preferred to receive some written information at least a few days prior to their operation. In a survey of 294 surgical patients by Oberle et al (1994) a large number of participants were dissatisfied with the timing of information provision, as the bulk of it occurred on the ward immediately prior to surgery. Prior to the rise in elective adult day surgery, timing of information delivery was not a problematic issue as patients were frequently admitted to hospital a number of days in advance of surgery. This has now changed and greater resources may now be required to ensure the early delivery of information.

Concerning discharge information provision, Otte (1996) revealed most patients preferred an early discharge although only when provided with adequate information. Martikainen et al (2000) investigated patient preference for anaesthesia when undergoing knee arthroscopy. Although this quasiexperimental research study was exploring anaesthetic drug preference, two unrelated aspects of patient dissatisfaction emerged i.e. not being able to speak with the surgeon prior to discharge and the lack of information. In a qualitative study by Kleinbeck and Hoffart (1994) participants were unsure of which 
activities they could perform around the house in the initial post-operative period. They would have preferred more information concerning recovery at home i.e. what activities could be undertaken and when. Donoghue et al (1997) following an in-depth interview of 21 participants reported many were satisfied with the information received. However, the lack of details regarding the possible problems encountered at home was a disadvantage. In a survey by Guilbert and Roter (1997) and a large audit by Hawkshaw (1994) it was stated patients coped well at home when discharge information provision was good i.e. explanations were given regarding simple daily activities.

A great deal of recovery now takes place at home following day surgery and the instant access to help and advice once available to the traditional surgical patient has been lost. Improved information provision to facilitate home recovery may now be urgently required. Information provision designed to aid recovery and provide explanations regarding the handling of perceived problems at home was strongly recommended. Some patients encountered events for which they felt unprepared, as they had not been informed of what to do should such an unforeseen event occur. Trial and error learning therefore frequently took place, as patients were uncertain of how best to proceed.

Patients' Experiences in the Day Surgery Unit (Table 5 p.118a). Two broad issues relate to patients' experience of day surgery and concern their expectations and level of anxiety. Firstly, issues concerning patient expectations. A large number of studies highlight the public's general satisfaction with, and preference for, day surgery (Clyne and Jamieson 1978, 
Jamison et al 1987, Harju 1991, O`Connor et al 1991, Buttery et al 1993, Fenton-Lee et al 1994, Ghosh and Sallam 1994, Gupta et al 1994, Sigurdardottir 1996, Lawrence et al 1997, Pollock and Trendholm 1997, Bhattacharya et al 1998, Willsher et al 1998). However, some aspects within day surgery were not always expected. In an audit by MacAndie and Bingham (1998) it was revealed a number of patients assumed day surgery to be minor surgery although they were surprised at having to walk to the operating theatre. Birch and Miller (1994) surveyed patients' attitudes towards walk-in surgery and found $98 \%$ expressed satisfaction for this approach although they were all still attending the hospital out-patient department at the time of data collection.

In an in-depth study to examine participation in decision-making, Avis (1994) revealed patients expected the doctors and nurses to make their choices for them as they viewed them as the experts. However, they also realised such expectations limited their involvement in the decision-making process. In a survey by Gnanalingham and Budhoo (1998) it was established, when allowed to make a choice, $91 \%$ of suitable patients preferred day surgery. However, only $33 \%$ chose to undergo local anaesthesia whereas $47 \%$ chose general anaesthesia. A greater proportion opting for general anaesthesia obviously demands a greater clinical and financial commitment. Also, it may be an indication of the increased anxiety associated with being awake and experiencing the operation i.e. patients may perceive general anaesthesia as less anxiety provoking as they are asleep while the surgical procedure takes place. This may be an unfavourable occurrence as not undergoing general 
anaesthesia prevents susceptibility to numerous additional risks i.e. $1: 350$ patients approximately, may experience light consciousness during general anaesthesia (Heier and Steen 1996).

The second and final aspect of patients' experience in day surgery concerns level of anxiety. Anxiety was discussed earlier in relation to its medical and nursing management. However, it is included again here as for some patients individual anxiety was a challenging issue. Eight studies reported waiting for surgery to commence following admission to be a problem for patients as it led to an increase in anxiety. Pollock and Trendholm (1997) reported $20 \%$ had to wait more than 3 hours for their operation and Read (1990), Buttery et al (1993) Ghosh and Sallam (1994), Otte (1996) and Rudkin et al (1996) all reported anxiety was increased by this period of waiting. Also, Nkyekyer (1996) reported $34 \%$ of patients found the wait prior to surgery too long and O'Connor et al (1991) also reported $11 \%$ found the wait too long. As a consequence both Ghosh and Sallam (1994) and Otte (1996) recommended staggered admission times on the day of surgery.

Male (1981) and Wicklin and Forster (1994) concluded female patients together with patients undergoing local anaesthesia to be more anxious. Nkyekyer (1996) revealed 60\% of female participants witnessed the care of an unconscious patient and of these $46 \%$ were made more anxious by the experience. However, these patients underwent day surgery under sedation and not full general anaesthesia. In a survey by Nyamathi and Kashiwabara (1988) it was established that the pre-operative cognitive abilities of all patients 
on the day of surgery were reduced as a result of anxiety. However, in order to determine this, participants were asked to complete a self-rating anxiety questionnaire plus a 100 -item critical thinking test immediately prior to surgery and general anaesthesia.

In a survey on the eve of surgery, Jamison et al (1987) examined the psychological factors believed to strongly influence recovery i.e. mood, fears and concerns. It was concluded patients who were more anxious preoperatively were prone to a greater number of post-operative complications. Moreover, patients who had a greater number of negative feelings about their recovery also experienced a slower recovery. In a survey by Daoud and Hasan (1999), using a visual analogue scale to measure anxiety and the ability of the medical staff to induce anaesthesia i.e. ease of intubation, a link between increased anxiety and additional difficulty with induction of general anaesthesia was established. It was concluded the more anxious patient could be more difficult to anaesthetise. However, no recommendations were made concerning the clinical application of such findings.

Privacy within the day surgery facility was also a recurrent problem and led to an increase in anxiety e.g. personal details being discussed in an open ward behind curtains, rectal suppositories being given intra-operatively without prior discussion and little privacy in the recovery area (Buttery et al 1993, Ghosh and Sallam 1994, Royal College of Surgeons of England and East Anglia R.H.A. 1995). Improvements were therefore recommended in order to protect patients' privacy, reduce the associated anxiety and improve satisfaction with care. 
Four studies revealed some patients were not happy to go home following their surgery as they were anxious regarding self care and would therefore have preferred an overnight stay (Pineault et al 1985, O'Connor et al 1991, Ratcliffe et al 1994, Nkyekyer 1996). In a comparatively older survey by Pineault et al (1985), 54\% of day-case patients believed their stay to be too short as opposed to $21 \%$ of the in-patients who also believed their stay was too short i.e. even a few nights in hospital was considered too short by some patients. Michaels et al (1992) revealed $74 \%$ of patients would have preferred an overnight stay and in a survey by Ratcliffe et al (1994) $8 \%$ of day-case patients would have preferred an overnight stay. O'Connor et al (1991) concluded male patients might prefer day surgery more than female patients as $16 \%$ of female patients preferred an overnight stay. This figure increased further in a study by Nkyekyer (1996), as $52 \%$ of female patients would have preferred an overnight stay because pain and the associated anxiety were highly problematic issues. However, all patients in the study by Nkyekyer (1996) had undergone surgery with intravenous sedation and local anaesthesia. Such a method of anaesthesia may have influenced the level of discomfort as a greater degree of pain was reported.

Certain expectations regarding day surgery were plainly incorrect and may have been indicative of i) the lack of opportunity in the pre-operative phase to undertake primary and secondary appraisal of the day surgery environment and, ii) the lack of comprehensive, accurate information. Additionally, numerous aspects increased anxiety for many patients i.e. waiting, the unfamiliar situation, 
the lack of privacy and the thought of coping at home following surgery. Again, the common theme throughout this review is repeated here - information provision. Had such patients been better informed or prepared more effectively for their day surgery experience i.e. improved problem focused and emotional focused coping strategies, numerous anxiety provoking aspects may have presented as less of a challenge.

Recovery at Home (Table 6 p.123a).

The final theme relates to the first few days and weeks at home and concerns morbidity (medical complications in the post-operative period), recovery behaviour and involvement of community healthcare professionals. The first issue concerns the plethora of studies examining post-operative morbidity. The degree of interest in morbidity helps to vividly demonstrate how the advancements in day surgery, outlined in Chapter 1, have had a strong medical focus. Numerous studies highlight the issue of post-operative morbidity although focus mainly upon the degree and duration of morbidity. Little or no attention is given to the information provided to patients regarding the 'expected' level of post-operative morbidity e.g. expected level and duration of pain. When a problem is expected via problem focused coping information, planned action can be undertaken i.e. problem focused coping. When an aspect of recovery is expected and planned, arguably the patient may be more effectively prepared.

In one of the earliest day surgery studies, Towey et al (1979) found no difference between two methods of induction of anaesthesia although the high level of post-operative abdominal pain was unexpected. Cundy and Read 
(1981) also reported no significant difference between two methods of induction of anaesthesia i.e. Methohexitone $120 \mathrm{mgs}$ and Thiopentone $300 \mathrm{mgs}$, on the degree of morbidity. Both studies however, utilised patients undergoing gynaecological surgery. This type of surgery, as will be discussed, has a tendency to give rise to a greater level of post-operative morbidity i.e. pain, nausea and vomiting.

Ghosh and Sallam (1994) reported one of the main sources of dissatisfaction in the post-operative period to be inadequate pain relief and in audits by Clyne and Jamieson (1978) and Birch and Miller (1994), 50\% of patients experienced pain while at home. Firth (1991), although utilising a short questionnaire within the audit process, discovered $25 \%$ of patients were awake and in pain during their first post-operative night. Thirty-one percent gained no relief or only partial relief when using the prescribed drugs. Fraser et al (1989) in a comprehensive survey interviewed 50 gynaecological patients and established the greatest amount of pain was experienced on the first post-operative day. ".... 51.6\% of the women utilised at least $50 \%$ of their prescribed number of tablets prescription range 10 - 30 tablets." (p. 194). Both Thatcher (1996) and Callesen et al (1998) reported the recommended or prescribed analgesia did not always bring pain relief. Donoghue et al (1995), utilising in-depth interviews, noted that most female patients did not expect the severity and duration of pain experienced. Both Edwards et al (1991) and Mackintosh and Bowles (1998) reported female patients undergoing gynaecological surgery experienced a great deal of pain although patients undergoing a variety of gynaecological 
surgery were included in the survey. Haldane et al (1998), Codd (1991) and Agboola et al (1998) all reported higher pain levels following gynaecological surgery and therefore made specific recommendations for this group i.e. greater use of analgesia and improved information.

In a survey by Smith (1998), during the first post-operative week, an increase in the level of pain for the under 65 year old age group was observed. A quasiexperimental study by Coslow and Eddy (1998) noted a greater number of patients demanded analgesia when not provided with information in the preoperative phase. However, the experimental group had a planned programme of education spanning 1 - 2 weeks. This is in sharp contrast to the control group who only received information 1 hour prior to surgery. Claxton et al (1997), employing a telephone survey 24 hours after surgery, established Morphine to be more effective than Fentanyl (both strong analgesics). It was concluded patients who were administered intravenous Fentanyl during surgery may have a greater need for supplementary oral analgesia in the first 24 hours following surgery. However, participants again underwent differing surgical procedures. Aasboe et al (1998) administered Betamethasone $12 \mathrm{mg}$ (steroid antiinflammatory) for pain management prior to surgery in a double-blind experimental study investigating post-operative pain. Positive results were established using this technique and it was recommended more studies should be conducted to gauge the beneficial effects of corticosteroids.

In a study by Jennings and Sherman (1987), 95\% of patients surveyed on the day of surgery and again one week later, felt they had not been provided with 
sufficient analgesia. Again, patients undergoing a variety of surgical procedures participated, which could lead to differing conclusions as pain levels may fluctuate depending upon the type of surgery. Limb et al (2000) studied 62 patients undergoing day surgery for haemorrhoidectomy. A multi-modal analgesia technique was utilised i.e. combination of two or more drugs and/ or two or more methods of delivery, to improve analgesia and minimize side effects. Ninety-five percent of patients were satisfied with their pain management. Implicit within this method of pain management, as it was a new day-case procedure, was the added information provided to patients regarding pain management.

Audits by Lewin and Razis (1995), Marquardt and Razis (1996) and Haddock et al (1999) concluded post-operative pain management was a considerable problem and recommended pre-packed analgesia plus relevant information to be provided on discharge. An audit by Fenton-Lee et al (1994) established 96\% of patients were satisfied with their post-operative pain management although in this study a community liaison sister visited during the immediate post-operative period for wound management and to provide advice i.e. additional information. In a further audit utilising a community liaison nurse, Ismail (1997) discovered $94 \%$ of patients did not require analgesia on the first night and only $60 \%$ used the 5-day supply of analgesia provided at discharge. An audit by Ramachandra (1994) revealed $69 \%$ of patients who had undergone a variety of operations experienced little or no pain in the post-operative period and an audit by Kangas-Saarela et al (1999) established 31\% of patients had no pain 24 hours 
after their operation. In a quasi-experimental design by Hulme et al (1999), patients who had received 5 minutes of foot massage from the nurses in the immediate post-operative period stated they too experienced less pain. However, the experimental group gained extra time with the nurse during the foot massage treatment, which may have enhanced information provision and self-efficacy appraisal. The control group received no placebo attention.

In a quasi-experiment by Alkaissi et al (1999) the effectiveness of nausea relieving pressure wristbands (SeaBands ${ }^{\circledR}$ ) was evaluated. In this double blind trial participants wearing the SeaBands ${ }^{\circledR}$ reported significantly less nausea and vomiting over a 24 hour period. In a further quasi-experiment by Parlow et al (1999) patients were randomised, prior to laparoscopic surgery and general anaesthesia, into two groups. Group one received a prophylactic intra-muscular injection of Promethazine (anti-emetic) while the second group received a placebo intra-muscular injection (normal saline). No differences were established between the two groups concerning the level of nausea, vomiting or rescue anti-emetics administered. However, patients identified as experiencing higher levels of nausea and vomiting in the recovery room continued to experience higher levels throughout the first 24-hour post-operative period. It was therefore recommended to target the highly nauseated patients in the recovery room for prophylactic anti-emetic therapy.

It is evident from the large number of studies in this section that much attention has focused upon the reduction of morbidity i.e. mainly pain management. This is a central medical issue as pain, nausea and vomiting are 
the primary reasons why patients are admitted to an in-patient hospital bed following day surgery (Mitchell et al 1999). Admission to an in-patient bed obviously defeats the object of day surgery as it increases overall costs. Again, a central aspect of pain management is the expectation of pain and information regarding pain management. Patients frequently do not expect the severity and duration of pain experienced. A number of studies indicate that patients can take proactive steps if the degree and duration of pain are expected (Clyne and Jamieson 1978, Firth 1991, Birch and Miller 1994, Donoghue 1995, Thatcher 1996, Callesen et al 1998).

The second issue regarding discharge home concerns recovery behaviour. Once discharged from the hospital Gupta et al (1994) discovered that some patients drove home (4\%) and many went home unaccompanied by an adult. Twenty-five percent were alone during the first 24 hours and $8 \%$ alone during the first 24 hours without an adult to look after their children. An audit by Birch and Miller (1994) revealed $13 \%$ of patients drove their car the same day and the majority returned home alone. Kelly (1994) reported, utilising a short questionnaire, that $7 \%$ drove their car on the first night of discharge, $42.7 \%$ reported feeling drowsy and $38.8 \%$ had a headache. "There was a wide distribution in the time to recover to full normal daily activity, ranging from the day of operation in 4 patients, one to two days in 45 , three to five days in 33 and six days or more in 21 patients." (p. 29).

In a large survey of 1,511 patients by Philip (1992) it was revealed the main post-operative problems were muscle aches, sore throat and drowsiness. An 
audit by Clyne and Jamieson (1978) reported 52\% of patients stayed off work for more than one week. However, post-operative recovery rates may differ widely as Ratcliffe et al (1994) and Wilkinson et al (1992) established 75\% and $84 \%$ of patients, respectively, still had problems 3 days after their operation. Conversely, an audit by Stephenson (1990) reported that almost 50\% of day surgery patients were active on the second post-operative day. Philip (1992) reported $32 \%$ of patients resumed normal activities the next day with a further $62 \%$ after 3 days.

Frisch et al (1990) conducted one of the few surveys, which also asked the carers to complete a questionnaire concerning their experiences of tending for a relative following day surgery. More than $30 \%$ of the patients required help with activities of daily living during the first 7 days although "Helpers tended to overestimate the patients' need for assistance." (p. 1006). This was mainly evident in the increased level of help believed to be required during bathing and the amount of pain believed to be experienced. However, some of the morbidity issues may have resulted from the participants all undergoing orthopaedic surgery i.e. greater morbidity may be associated with this type of surgery (Frisch et al 1990). A survey by O'Connor et al (1991) reported $62 \%$ of patients required a carer for one day or less and $20 \%$ for 1 - 2 days. Female patients required more assistance than males with $3 \%$ of females paying someone to help with childcare and housework. However, no consideration was given to the differing types of surgery undertaken. In a comprehensive survey by the Royal College of Surgeons of England and East Anglia R.H.A. (1995) more than one 
third of patients required a great deal of support from helpers at home, $20 \%$ of whom had to take time off work. Willis et al (1997) established $21 \%$ of patients required help from carers, $10 \%$ of whom had to take an average of 3 days off work with $7 \%$ losing earnings. In an audit by Petticrew et al (1995), utilising a large sample from 35 N.H.S. hospitals, almost $33 \%$ of patients required 'quite a lot' or 'a great deal' of help from relatives or friends following discharge.

Many patients evidently do not follow the instructions provided once discharged home following day surgery. Patients driving home following general anaesthesia is of great concern as such patients are potentially under the influence of drugs while driving. The number of patients at home caring for themselves and attempting to gain full recovery with limited information is clearly evident. If in the future additional in-patient surgery is to be converted into day-case surgery, the need for improved information provision will be considerable. Moreover, the continued expansion of day surgery clearly depends upon willing and able lay-people to care for their relatives/ friends. This is frequently at some financial and emotional expense to themselves. In such an uneasy domestic situation a dearth of information may only seek to exacerbate such problems.

The final aspect of recovery relates to the involvement of healthcare professionals. To enable both patients and their carers to gain much needed advice following discharge eight studies recommend the use of telephone helplines (Kempe and Gelazis 1985, Kleinbeck and Hoffart 1994, Lewin and Razis 1995, De Jesus et al 1996, Wedderburn et al 1996, Willis et al 1997, 
Heseltine and Edlington 1998, MacAndie and Bingham 1998). Moreover, in an in-depth study by Donoghue et al (1995) data collection partially involved a telephone interview. The telephone interview was viewed in itself to be a positive experience for patients as Donoghue et al (1995) states "There seemed to be a therapeutic factor embedded within the interview process for some women." (p. 176).

The level of community healthcare involvement was reported in thirteen studies. These mainly concerned visits to the general practitioner or visits by the district nurse. In a survey by Kennedy (1995) 93\% of the patients, although having undergone a moderate surgical procedure and general anaesthesia, did not seek community-based help in the first three post-operative days. Birch and Miller (1994) found only $19 \%$ had contacted their general practitioner within the first 2 weeks and King (1989) revealed in an audit that only 5\% of patients required help in the first 48 hours. A survey by Wedderburn et al (1996), utilising a very brief questionnaire, established $19 \%$ of patients had to visit their general practitioner at least once regarding pain or wound management. In an Australian survey by Singleton et al (1996), it was revealed $21 \%$ of patients contacted their general practitioner within the first 2 weeks regarding pain management or wound care and the district nurses were required to visit patients an average of 2 - 3 times in the post-operative period. In an audit by Woodhouse et al (1998) it was discovered $18 \%$ of patients had visited their general practitioner for either a medical certificate or wound care advice although $70 \%$ of the general practitioners felt day surgery had not caused a 
significant increase in workload. An audit by Thomas and Hare (1987) reported general practitioners were satisfied with day surgery and gave "...wholly favourable comments." (p. 447). Many patients remained self-sufficient although many contacted their general practitioner or community nurses for additional help or information (Thomas and Hare 1987).

As more complex surgical procedures are undertaken on a great number of day surgery patients, the level of community activity concerning day surgery patients will inevitably increase (Singleton et al 1996, Jarrett 1997). In a survey by Michaels et al (1992) utilising a single-page questionnaire it was established day surgery patients required more medical attention following discharge. However, the survey compared participants undergoing in-patient surgery with participants undergoing day-case surgery. Without the extra advice and communication predictably gained from spending a greater amount of time in hospital, the day-case patient would naturally require increased contact with the general practitioner in the post-operative period. Likewise, in studies by the Royal College of Surgeons of England and East Anglia R.H.A. (1995) and Willis et al (1997) almost half of the patients required help from one community healthcare agency. Furthermore, in a recent study by Hunt et al (1999) involving a relatively new day-case procedure (haemorrhoidectomy), $86 \%$ of participants were very satisfied with day surgery although a registered nurse visited all patients following discharge. Some patients required nine separate visits.

Again, this final aspect emphasises the benefits to be gained when patients are able to speak with and gain information from healthcare professionals in the 
post-operative period. Telephone helplines have been widely recommended as an effective way of providing additional information together with home visits by district nurses.

In summary, a review of the literature revealed satisfaction within day surgery to be very high although four main themes emerged i.e. nursing and anaesthetic practice, information provision, experiences in the day surgery unit and recovery at home. Within nursing and anaesthetic practice there was a strong requirement for the establishment of pre-assessment clinics to increase patient contact time, improve communication and help allay fears. A number of measures attained moderate success in anxiety management i.e. early contact, relaxation, distraction, therapeutic role of the nurse and positive, encouraging statements. Other practices requiring consideration related to patient discharge information covering common unforeseen events.

Issues surrounding the provision of information by far presented the greatest challenge and were implicit within all the themes. A general lack of information was a common element, especially within mixed day surgery facilities i.e. day surgery patients within in-patients wards. However, not all patients wanted the same level of information, as some were made more anxious when provided with too much information and others more anxious with too little information.

Information relevant to home recovery i.e. management of the wound, daily activity level, what to do during an unforeseen event, were all frequently cited as being most useful. Videotaped/ audiotaped presentations had some success and information provision prior to admission was widely viewed as positive. 
Patients' experience of day surgery concerned realistic expectations of procedures on the day, level of pain and incapacitation following surgery. Much anxiety was generated by the time spent waiting for surgery following admission, the lack of privacy, the prospect of undergoing any form of anaesthesia and recovery at home.

Finally, concerning recovery at home, pain management was a considerable problem. This was a particular problem for gynaecological patients as ineffective pain management generated much dissatisfaction and increased anxiety. This may be indicative of a lack of adequate preparation. Once discharged, many carers were happy with their role although more information was frequently required and more time in which to make social arrangements to accommodate their temporary role. There was generally a small increase in workload for general practitioners and district nurses although if the level and complexity of day surgery activity were to increase, this may change.

As suspected, information provision was therefore a major challenge for modern day surgery. If evidence can be gained for the psycho-educational plan of support, outlined in Chapter 2, tremendous advances in pre-operative psychological care may be possible for the adult patient undergoing modern elective day surgery. 


\section{Chapter 5}

\section{Psychological Preparation for Patients Undergoing Day Surgery}

\section{Methodology}

\section{Study Overview}

The research question was 'What are the effects when two differing levels of information are randomly provided to participants undergoing day surgery who possess a vigilant or avoidant coping style'. The assertion being that if a participant receives the information level which matches their coping style then certain aspects, crucial to recovery (Chapter 2), will be positively influenced i.e. satisfaction with information provision would increase, health locus of control would be positively influenced, self-efficacy would be enhanced and anxiety reduced. Differing levels of information distributed to participants at random would inevitably lead to some receiving a mismatch between their individual coping style and desired level of information i.e. some vigilant copers would be in receipt of the simple information booklet and some avoidant copers would be in receipt of the extended information booklet. However, such a design was required in order to verify that participants in the mismatch group would indeed experience more negative outcomes in comparison to participants in the matched group.

A quasi-experimental research design was employed on a convenience sample of women undergoing elective gynaecological day surgery and general anaesthesia. Participants were contacted by telephone prior to surgery in order 
to explain the purpose of the study, gain consent and confirm the date for surgery. Random assignment into two experimental groups then followed. Two groups of participants were necessary as a between subjects or unrelated factorial research design was utilised to examine the differing effects the independent variables (coping style $\mathrm{X}$ information booklet received) exerted on the dependent variables (anxiety, self-efficacy, healthcare control, satisfaction and nurses' ratings of adjustment to surgery) (Design section). Participants who were scheduled to have their surgery on the same day were sent the same information booklet in order to avoid possible contamination of results i.e. ensure independence of participant reporting. The information was posted to participants 7 to 10 days in advance of their surgery as receiving information on the day of surgery has been observed to arrive too late to make any appreciable difference (Young et al 1994). An overall satisfaction with information interview then took place by telephone 2 - 4 days following surgery (Design section).

The overall plan was therefore initial contact, information booklet and questionnaires sent 7 to 10 days in advance of surgery, intervention (laparoscopic day surgery and general anaesthesia) and finally completion of post-operative questionnaire 2 - 4 days following surgery. Day surgery patients were to be the focus as previously no formal programmes of psychoeducational nursing intervention had been implemented in day surgery. During the last 10 to 15 years day surgery has become an integral part of surgical healthcare within the National Health Service. Day surgery fundamentally involves minimal contact with hospital personnel. This central problem 
exacerbates the issue of psycho-educational intervention. Furthermore, as day surgery expands both in real terms and as part of the total surgical workload, issues relating to information provision will grow and remain a constant challenge. In order to explain a number of terms relating to such issues in day surgery and the study in general, some definitions are provided for clarification (Table 7 p.138). 


\begin{tabular}{|c|c|}
\hline TERM & DEFINITION \\
\hline $\begin{array}{l}\text { Ambulatory Surgery or } \\
\text { Day Surgery }\end{array}$ & $\begin{array}{l}\text { Surgical treatment undertaken in less than } 8 \text { hours with no overnight } \\
\text { stay although the patients will require a hospital bed (UK definition). } \\
\text { Synonyms - day surgery, same day surgery, day only. }\end{array}$ \\
\hline $\begin{array}{l}\text { Procedural Laparoscopic } \\
\text { Gynaecological Surgery }\end{array}$ & $\begin{array}{l}\text { Fine telescopic like instrument inserted into the pelvic cavity in order to } \\
\text { view the anatomical structures and undertake a surgical procedure. }\end{array}$ \\
\hline $\begin{array}{l}\text { Diagnostic Laparoscopic } \\
\text { Gynaecological Surgery }\end{array}$ & $\begin{array}{l}\text { Fine telescopic like instrument inserted into the pelvic cavity in order to } \\
\text { view the anatomical structures and perform a test[s] to aid a medical } \\
\text { diagnosis. }\end{array}$ \\
\hline $\begin{array}{l}\text { Procedural } \\
\text { Information }\end{array}$ & $\begin{array}{l}\text { The sequential order of events on the day of surgery once admitted to } \\
\text { the surgical unit i.e. what will happen next and the order in which the } \\
\text { events will occur. }\end{array}$ \\
\hline $\begin{array}{l}\text { Behavioural } \\
\text { Information }\end{array}$ & $\begin{array}{l}\text { The behaviour[s] or action[s] the patient is required to undertake either } \\
\text { before, during or after the surgical procedure i.e. adopting a certain } \\
\text { position for the procedure, gentle movements only, deep breathing, } \\
\text { etc.. }\end{array}$ \\
\hline $\begin{array}{l}\text { Sensory } \\
\text { Information }\end{array}$ & $\begin{array}{l}\text { The bodily sensations the patient is likely to experience before, during } \\
\text { or after the surgical procedure i.e. the likely sensations during } \\
\text { anaesthesia, degree and duration of pain, etc.. }\end{array}$ \\
\hline $\begin{array}{l}\text { Cognitive or } \\
\text { Emotional Coping } \\
\text { Strategies } \\
\end{array}$ & $\begin{array}{l}\text { The positive thoughts a patient can draw upon in order to gain } \\
\text { assurance they will be safe, awake from their operation, be unharmed } \\
\text { and gain a full recovery i.e. being told of the highly trained staff, fast } \\
\text { effective drugs, modern equipment, etc.. }\end{array}$ \\
\hline Vigilant Coping & $\begin{array}{l}\text { Coping style for dealing with an aversive situation characterised by the } \\
\text { desire for maximum levels of information. The provision of too little } \\
\text { information may give rise to an increase in anxiety. }\end{array}$ \\
\hline Avoidant Coping & $\begin{array}{l}\text { Coping style for dealing with an aversive situation characterised by the } \\
\text { desire for minimum levels of information. The provision of too much } \\
\text { information may give rise to an increase in anxiety. }\end{array}$ \\
\hline Fluctuating Coping & $\begin{array}{l}\text { Coping style for dealing with an aversive situation characterised by the } \\
\text { desire for variable levels of information. The information desired may } \\
\text { be highly specific. Incorrect communication of the desired amount or } \\
\text { selected areas of information may give rise to an increase in anxiety. }\end{array}$ \\
\hline Flexible Coping & $\begin{array}{l}\text { Coping style for dealing with an aversive situation characterised by } \\
\text { assuming an adaptable stance regarding information provision. } \\
\text { Generally, whatever information is provided will be acceptable. }\end{array}$ \\
\hline $\begin{array}{l}\text { Extended Information } \\
\text { Booklet }\end{array}$ & $\begin{array}{l}\text { An informative, educational booklet i.e. full explanations, diagrams and } \\
\text { overall, empowering. This was to be targeted at vigilant copers who } \\
\text { generally prefer more detailed information. }\end{array}$ \\
\hline $\begin{array}{l}\text { Simple Information } \\
\text { Booklet }\end{array}$ & $\begin{array}{l}\text { A directing, uncomplicated booklet i.e. short explanations, no } \\
\text { diagrams and largely instructional. This was to be targeted at avoidant } \\
\text { copers who generally prefer a less detailed level of information. }\end{array}$ \\
\hline
\end{tabular}




\section{Hypotheses}

The main research question was 'What are the effects when two differing levels of information are randomly provided to participants undergoing day surgery who possess a vigilant or avoidant coping style'. In order to test the research question five hypotheses were constructed. Each hypothesis is detailed below followed by the projected theoretical relationship between the independent variables and dependent variable in plot graph format. Plot graphs have been utilised as they help to clearly display the $2 \times 2$ factorial design (Design section). Additionally, plot graphs provide a clear demonstration of the predicted main effects i.e. the overall effect an independent variable may exert, and the predicted main interaction effects i.e. influence an independent variable may have upon one level of the other independent variable.

\section{Hypothesis One}

When an individual with -

a) a vigilant coping style receives an extended level of pre-operative information, they will be more satisfied with the information than a vigilant coper in receipt of a simple level of pre-operative information.

b) an avoidant coping style receives a simple level of pre-operative information, they will be more satisfied with the information than an avoidant coper in receipt of an extended level of pre-operative information.

Satisfaction with the information was considered in four ways i.e. satisfaction with the information booklet, satisfaction with information provision overall, the amount of information requested and the nurses' rating of the amount of 
information deemed to be required. Firstly, satisfaction with the information booklet will be considered.

A vigilant coper may experience reduced satisfaction when in receipt of the simple information booklet as too little educational material may cause an increase in anxiety and lead to much negative rumination. An increase in satisfaction may therefore occur for a vigilant coper when in receipt of the extended information booklet. Conversely, an avoidant coper may experience reduced satisfaction when in receipt the extended information booklet as too much information may cause an increase in anxiety. An increase in satisfaction for an avoidant coper may therefore occur when in receipt of the simple information booklet (Figure 2).

FIGURE 2

PLOT OF POSSIBLE THEORETICAL RELATIONSHIP BETWEEN INDEPENDENT VARIABLES (INFORMATION PROVIDED X COPING STYLE) AND DEPENDENT VARIABLE (SATISFACTION WITH INFORMATION BOOKLET).

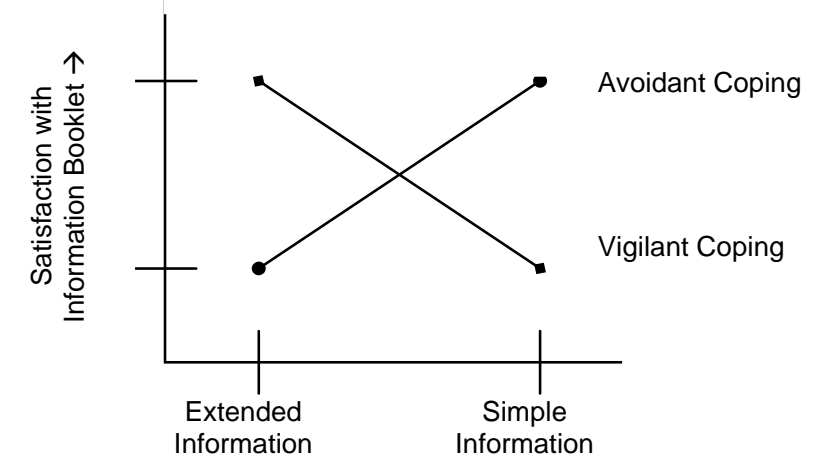

Secondly, satisfaction with information provision overall may be reduced for a vigilant coper when in receipt of the simple information booklet, little information from the day surgery unit and little post-discharge information. Such a reduced amount of educational material may cause an increase in anxiety and lead to much negative rumination. Satisfaction may therefore increase for a vigilant 
coper when in receipt of the extended information booklet, a large amount of information from the day surgery unit and a large amount of post-discharge information. Conversely, satisfaction with information provision overall may be reduced for an avoidant coper when in receipt of the extended information booklet, a large amount of information from the day surgery unit and a large amount of post-discharge information. Such a level of information provision may lead to an increased level of anxiety. Satisfaction may therefore be increased for an avoidant coper when in receipt of the simple information booklet, little information from the day surgery unit and little post-discharge information. This may lead to a reduced level of stress, as no anxiety provoking or unwanted educational material will be viewed (Figure 3).

FIGURE 3

PLOT OF POSSIBLE THEORETICAL RELATIONSHIP BETWEEN INDEPENDENT VARIABLES (INFORMATION PROVIDED X COPING STYLE) AND DEPENDENT VARIABLE (SATISFACTION WITH INFORMATION).

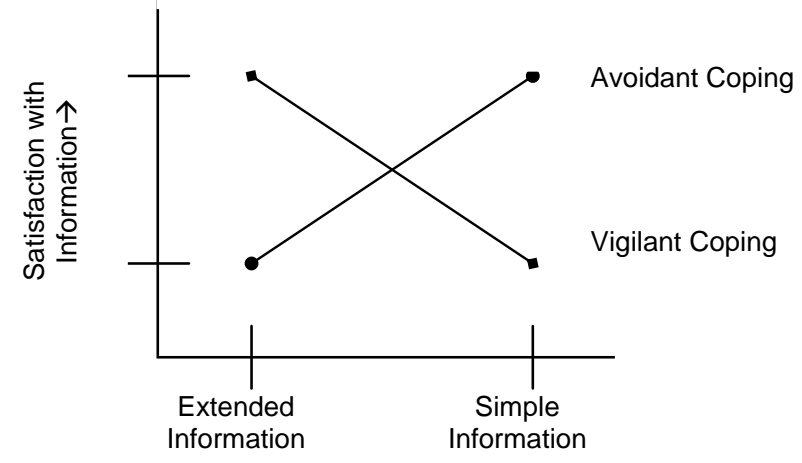

The third aspect concerns the amount of information requested. A vigilant coper may appraise the amount of information required as being higher when in receipt of the simple information booklet as too little is available. When a vigilant coper is in receipt of the extended information booklet appraisal of information required may be lower, as the educational material provided may be deemed 
sufficient. Information required by an avoidant coper might demonstrate little appreciable difference, as the level desired was potentially accessible via both booklets (Figure 4).

FIGURE 4

PLOT OF POSSIBLE THEORETICAL RELATIONSHIP BETWEEN INDEPENDENT VARIABLES (INFORMATION PROVIDED X COPING STYLE) AND DEPENDENT VARIABLE (INFORMATION REQUESTED).

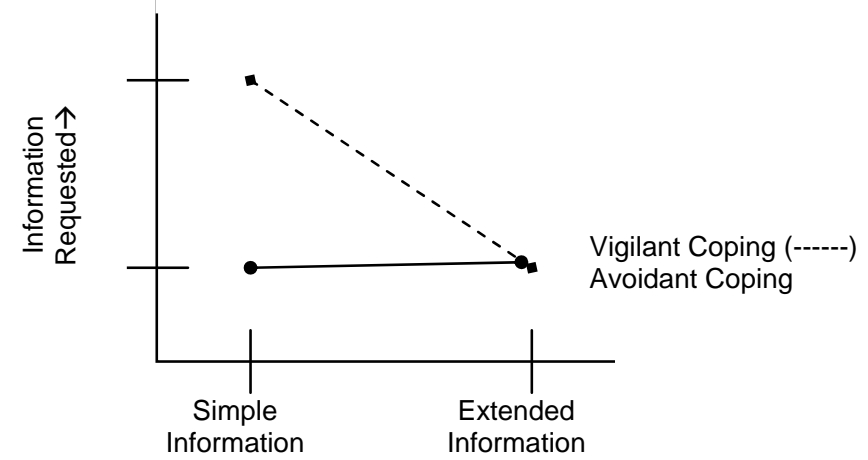

The final aspect within hypothesis one concerns the overall amount of information assessed as being required by the nurses. An increased level of information may be assessed as being required, for a vigilant coper when in receipt of the simple information booklet, as they may ask more questions and seek more information. A decreased level of information may be assessed as being required, for a vigilant coper in receipt of the extended information booklet, as they may ask fewer questions and seek little extra information. Information required by an avoidant coper might demonstrate little appreciable difference, as the desired level of information was potentially accessible via both booklets (Figure 5 p.143). 
FIGURE 5

PLOT OF POSSIBLE THEORETICAL RELATIONSHIP BETWEEN INDEPENDENT VARIABLES (INFORMATION PROVIDED X COPING STYLE) AND DEPENDENT VARIABLE (BEHAVIOURAL INFORMATION).

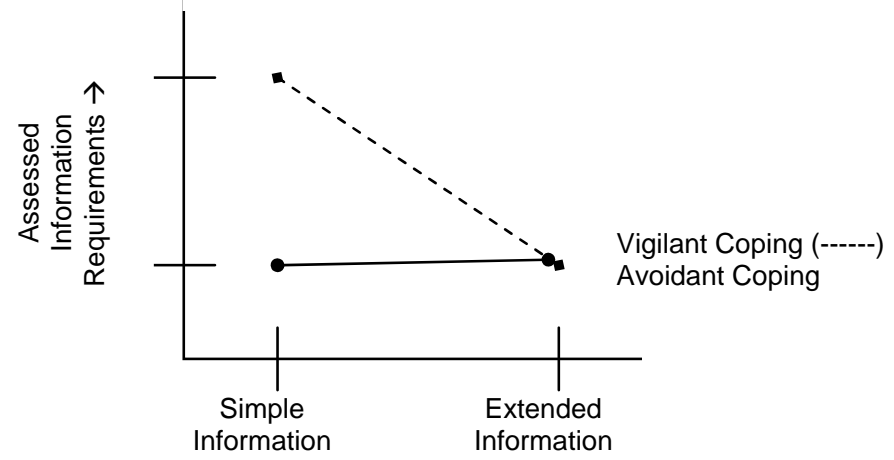

\section{Hypothesis Two}

When an individual with -

a) a vigilant coping style receives an extended level of pre-operative information, they will be less anxious than a vigilant coper in receipt of a simple level of pre-operative information.

b) an avoidant coping style receives a simple level of pre-operative information, they will be less anxious than an avoidant coper in receipt of an extended level of pre-operative information.

Participant anxiety was considered in two ways i.e. self-rated state anxiety and nurse-rated anxiety. Firstly, self-rated state anxiety will be considered. Anxiety for a vigilant coper may be increased when only in receipt of the simple information booklet as too little information may be available. A decrease in anxiety for a vigilant coper may therefore occur when in receipt of the extended information booklet. Conversely, anxiety for an avoidant coper may be increased when in receipt of the extended information booklet as too much 
information may be available. A decrease in anxiety may therefore occur for an avoidant coper when in receipt of the simple information booklet (Figure 6).

FIGURE 6

PLOT OF POSSIBLE THEORETICAL RELATIONSHIP BETWEEN INDEPENDENT VARIABLES (INFORMATION PROVIDED X COPING STYLE) AND DEPENDENT VARIABLE (SELF-RATED STATE ANXIETY).

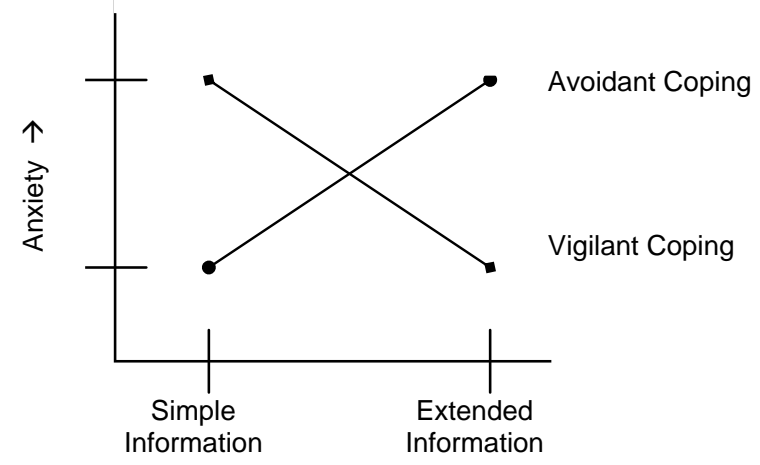

Secondly, an increase in anxiety may be observed in a vigilant coper by the nursing staff, when in receipt of the simple information booklet as only a small amount of information is available. A lower level of anxiety may therefore be observed in a vigilant coper when in receipt of the extended information booklet. Conversely, an increase in anxiety may be observed in an avoidant coper by the nursing staff, when in receipt of the extended information booklet as too much educational material is available. A lower level of anxiety may therefore be observed in an avoidant coper when in receipt of the simple information booklet (Figure 7 p.145). 
FIGURE 7

PLOT OF POSSIBLE THEORETICAL RELATIONSHIP BETWEEN INDEPENDENT VARIABLES (INFORMATION PROVIDED X COPING STYLE) AND DEPENDENT VARIABLES (BEHAVIOURAL ANXIETY).

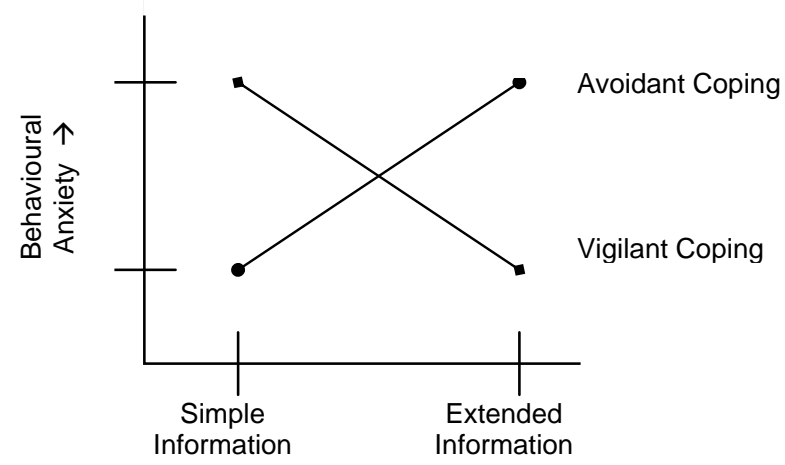

\section{Hypothesis Three}

When an individual with -

a) a vigilant coping style receives an extended level of pre-operative information, adjustment to surgery will be more positively influenced in comparison to a vigilant coper in receipt of a simple level of pre-operative information.

b) an avoidant coping style receives a simple level of pre-operative information, adjustment to surgery will be more positively influenced in comparison to an avoidant coper in receipt of an extended level of preoperative information.

A decreased adjustment to surgery may be observed for a vigilant coper by the nursing staff, when in receipt of the simple information booklet as greater anxiety and negative rumination may occur. An increase in adjustment for a vigilant coper may therefore be observed when in receipt of the extended information booklet. Conversely, a decrease in adjustment to surgery may be observed for an avoidant coper by the nursing staff when in receipt of the 
extended information booklet as too much information may cause greater anxiety. An increase in adjustment to surgery may therefore be observed for an avoidant coper when in receipt of the simple information booklet (Figure 8).

FIGURE 8

PLOT OF POSSIBLE THEORETICAL RELATIONSHIP BETWEEN INDEPENDENT VARIABLES (INFORMATION PROVIDED X COPING STYLE) AND DEPENDENT VARIABLE (OBSERVED ADJUSTMENT TO SURGERY).

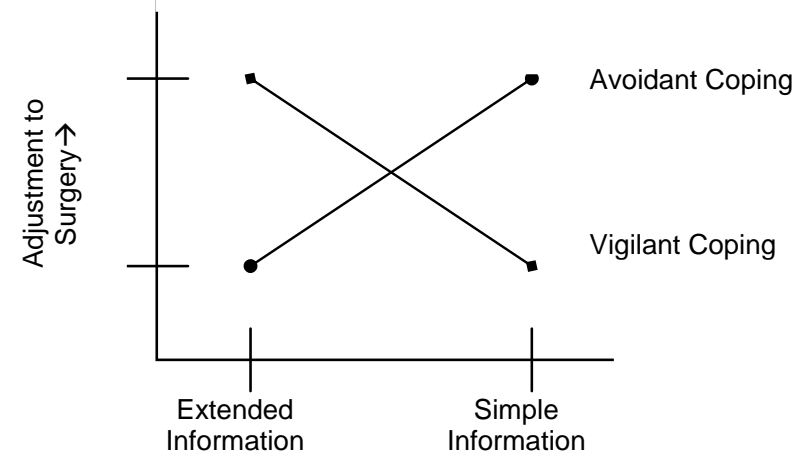

\section{Hypothesis Four}

When an individual with -

a vigilant or avoidant coping style receives the preferred level of preoperative information, the desired level of health control will be more readily attained in comparison to a vigilant or avoidant coper in receipt of the nonpreferred level of pre-operative information.

Participant desire for health control was considered in three ways i.e. internal health locus of control, chance health locus of control, external health locus of control (powerful others). Firstly, internal health locus of control will be outlined. A vigilant coper in receipt of the simple information booklet may experience a decrease in internal health locus of control, as too little information may be available to aid decision-making. Greater internal health locus of control appraisals may therefore occur for a vigilant coper in receipt of the extended 
information booklet. Conversely, internal health locus of control appraisals for an avoidant coper may be decreased when in receipt of the extended information booklet as such individuals may wish to have minimal knowledge and involvement in the decision-making process, preferring to trust in the 'experts'. Greater internal health locus of control appraisals for an avoidant coper may therefore occur when in receipt of the simple information booklet (Figure 9).

FIGURE 9

PLOT OF POSSIBLE THEORETICAL RELATIONSHIP BETWEEN INDEPENDENT VARIABLES (INFORMATION PROVIDED X COPING STYLE) AND DEPENDENT VARIABLE (INTERNAL HEALTH LOCUS OF CONTROL).

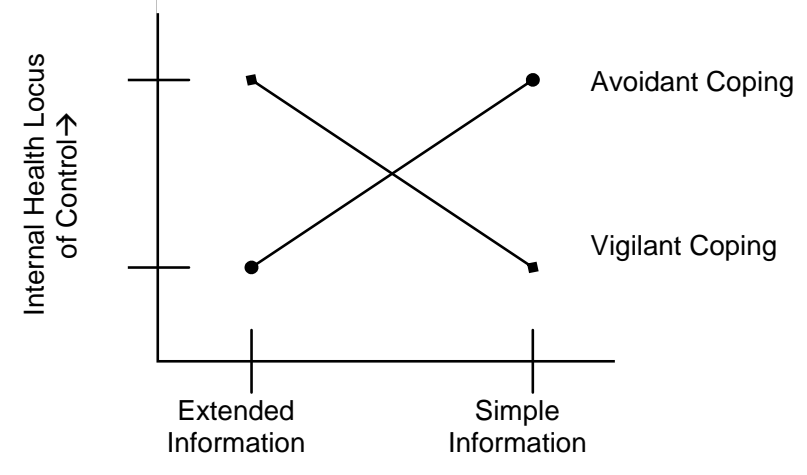

Secondly, chance health locus of control appraisals for a vigilant coper may be increased, when in receipt of the simple information booklet as too little may be known thereby providing little insight into events i.e. care, treatment and recovery. A decrease in chance health locus of control appraisal may therefore occur for a vigilant coper when in receipt of the extended information booklet. Conversely, chance health locus of control appraisals for an avoidant coper may be increased when in receipt of the extended information booklet as such individuals wish to have minimal involvement and knowledge of events, preferring to trust in the 'experts'. A decrease in chance health locus of control 
appraisals for an avoidant coper may therefore occur when in receipt of the simple information booklet (Figure 10).

FIGURE 10

PLOT OF POSSIBLE THEORETICAL RELATIONSHIP BETWEEN INDEPENDENT VARIABLES (INFORMATION PROVIDED X COPING STYLE) AND DEPENDENT VARIABLE (CHANCE HEALTH LOCUS OF CONTROL).

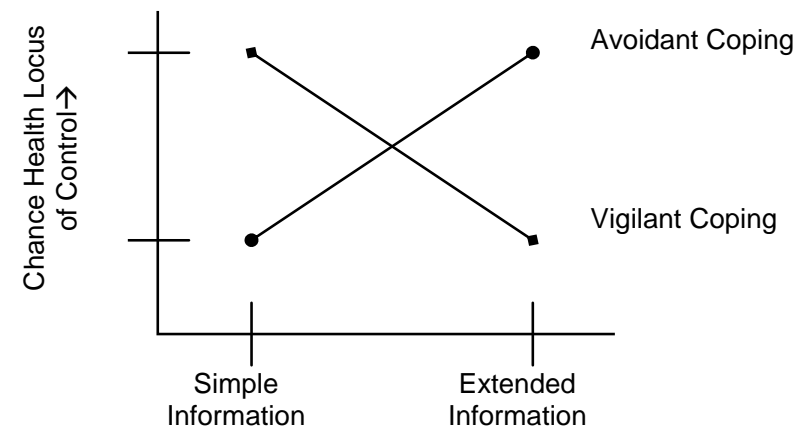

Finally, external health locus of control appraisals (powerful others) for a vigilant coper may be increased when in receipt of the simple information booklet as too little may be known. This may offer too little insight into events, limit participation in the decision-making process and permit too great an influence from powerful others. A decrease in external health locus of control appraisals (powerful others) may therefore occur for a vigilant coper when in receipt of the extended information booklet. Conversely, external health locus of control appraisals (powerful others) for an avoidant coper may be increased when in receipt of the extended information booklet as such individuals may wish to have minimal involvement in the decision-making process, preferring to trust in the 'experts'. A decrease in external health locus of control appraisals (powerful others) may therefore occur for an avoidant coper when in receipt of the simple information booklet (Figure 11 p.149). 
FIGURE 11

PLOT OF POSSIBLE THEORETICAL RELATIONSHIP BETWEEN INDEPENDENT VARIABLES (INFORMATION PROVIDED X COPING STYLE) AND DEPENDENT VARIABLE (EXTERNAL HEALTH LOCUS OF CONTROL).

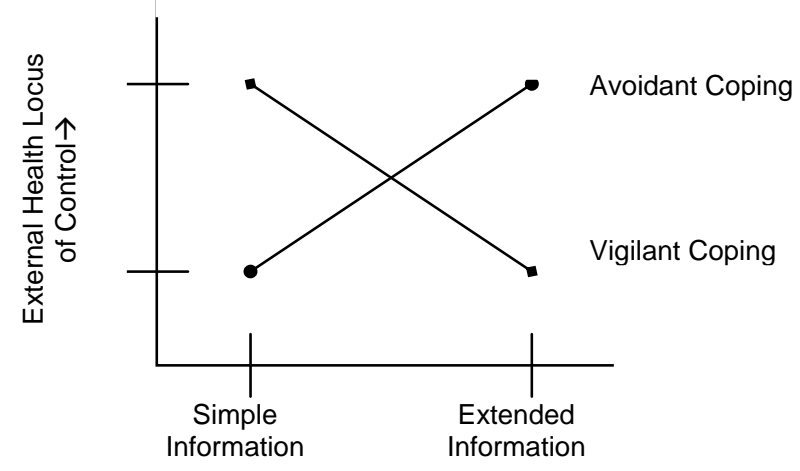

\section{Hypothesis Five}

When an individual with -

a vigilant or avoidant coping style receives the preferred level of preoperative information, the perceived ability to cope will be more readily attained in comparison to a vigilant or avoidant coper in receipt of the nonpreferred level of pre-operative information.

Self-efficacy appraisals for a vigilant coper may be reduced when in receipt of the simple information booklet as the small amount of information may hinder coping with admission to hospital and recovery at home i.e. all sudden and unpredicted events may promote negative appraisals and have the potential to influence coping ability (vigilant copers tend to evaluate all unforeseen events as potentially negative). An increase in self-efficacy appraisals may therefore occur for a vigilant coper when in receipt of the extended information booklet as they will be more informed of all events so that nothing surprises them. Conversely, self-efficacy appraisals for an avoidant coper may be reduced when in receipt of the extended information booklet as too much information 
may provide unwanted details. Avoidant copers prefer to minimise all events and be the passive recipients of care from the experts i.e. doctors and nurses. An increase in self-efficacy appraisals may therefore occur for an avoidant coper when in receipt of the simple information booklet (Figure 12).

\section{FIGURE 12}

PLOT OF POSSIBLE THEORETICAL RELATIONSHIP BETWEEN INDEPENDENT VARIABLES (INFORMATION PROVIDED X COPING STYLE) AND DEPENDENT VARIABLE (SELF-EFFICACY).

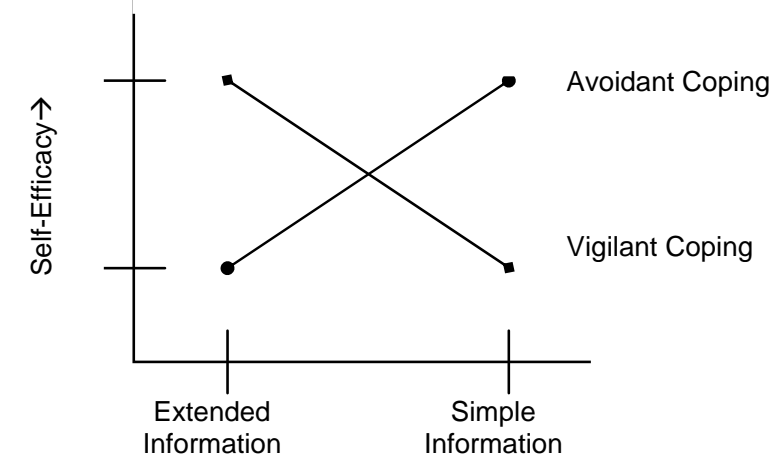

In summary, five hypotheses were explored to answer the research question. These hypotheses were -

1. When an individual with -

a) a vigilant coping style receives an extended level of pre-operative information, they will be more satisfied with the information than a vigilant coper in receipt of a simple level of pre-operative information.

b) an avoidant coping style receives a simple level of pre-operative information, they will be more satisfied with the information than an avoidant coper in receipt of an extended level of pre-operative information. 
2. When an individual with -

a) a vigilant coping style receives an extended level of pre-operative information, they will be less anxious than a vigilant coper in receipt of a simple level of pre-operative information.

b) an avoidant coping style receives a simple level of pre-operative information, they will be less anxious than an avoidant coper in receipt of an extended level of pre-operative information.

3. When an individual with -

a) a vigilant coping style receives an extended level of pre-operative information, adjustment to surgery will be more positively influenced in comparison to a vigilant coper in receipt of a simple level of preoperative information.

b) an avoidant coping style receives a simple level of pre-operative information, adjustment to surgery will be more positively influenced in comparison to an avoidant coper in receipt of an extended level of preoperative information.

4. When an individual with a vigilant or avoidant coping style receives the preferred level of pre-operative information, the desired level of health control will be more readily attained in comparison to a vigilant or avoidant coper in receipt of the non-preferred level of pre-operative information.

5. When an individual with a vigilant or avoidant coping style receives the preferred level of pre-operative information, the perceived ability to cope will 


\section{Hospital and Ethical Approval}

In order to access the sample of day surgery patients, written permission was gained from 5 Obstetric and Gynaecological Consultants who commonly utilised the Day Surgery Unit in a local N.H.S. Trust Hospital. Each surgeon was sent a copy of the research proposal together with the projected documentation i.e. information booklets, a covering letter with the researcher's name, address and contact telephone number, consent form and questionnaires (Appendices 3 to 12). Following some minor alterations to the information booklet, all surgeons agreed to the study, prior to ethical approval. Agreement was reached on the strict understanding the study would not hinder proceedings on the day of surgery. Also, upon conclusion of the study, the results would be made available for discussion. Permission was also gained at this stage from the Anaesthetic Directorate, Head Nurse within the Surgical Directorate and the Senior Nurse within the Day Surgery Unit. This process took many months as the booklets and questionnaires were discussed at numerous meetings i.e. Obstetric and Gynaecological Directorate, Anaesthetic Directorate, Nursing Directorate.

General agreement from the hospital staff for the study was therefore reached prior to submission of the proposal to the local Ethical Committee. The completed proposal together with the questionnaires, consent form, information booklets and hospital staff's letters of approval were submitted to the Ethical 
Committee. Following a few minor amendments approval was granted. The Ethical Committee requested no formal interview of the researcher and formal approval was given (Appendix 2).

\section{Participants}

The general hospital utilised was situated in an industrial location on the outskirts of a large city in the Northwest of England. The hospital was a large N.H.S. Trust University Teaching Hospital. A convenience sample of female patients scheduled to undergo elective gynaecological day surgery i.e. laparoscopic surgery, in the hospital's dedicated day surgery facility were the participant group. Only those participants who had not previously undergone day surgery within the last 12 months were utilised as any recent experience may have influenced their knowledge of events and possibly resulted in a more informed and less anxious participant. Also, only patients over the age of 18 years undergoing intermediate, non life-threatening surgery were included as the study was aimed solely at healthy, adult day surgery patients experiencing an acute, elective surgical episode. All participants had to be able to speak and read English. Furthermore, only patients who had no previous history of chronic mental or physical health problems were invited to take part in order to avoid the introduction of too many extraneous variables e.g. increased anxiety resulting from a chronic health problem or surgery for a suspected malignancy. This did not present as a problem however, as only patients who are physically and mentally fit are eligible to undergo day surgery. Approximately $n=250$ potential participants' names and telephone numbers were taken from the 
operating lists over a 10 month period although only $n=120$ were contactable. Of this number only $n=87$ were included in the study (Procedure section).

Laparoscopic gynaecological surgery is undertaken for two main reasons i) diagnostic purposes e.g. fertility problems, pelvic pain and, ii) treatment purposes e.g. sterilisation (render infertile by occlusion of both fallopian tubes). This group were chosen because of the large number of patients undergoing this type of intermediate surgery and the need to identify a specific, non lifethreatening, intermediate surgical procedure. As information was to be sent prior to the day of surgery, a specific operation had to be pre-selected so that an information booklet could be produced. Furthermore, female participants were targeted, as previously male participants had been extremely reluctant to volunteer for a study examining pre-operative preparation (Mitchell 1997).

\section{Materials}

The instruments employed within this study were two researcher designed information booklets, four established questionnaires and two researcherdesigned questionnaires. Each will be discussed in this order. Firstly, the information instruments were two Laparoscopy Information Booklets (Appendices $3 \& 4$ ). The booklets were designed by the researcher to contain an extended level of information and a simple level of information with vigilant and avoidant copers, respectively, being the target recipients. What constitutes 'extended' information provision and 'simple' information provision was established via extensive reading of current hospital/ day surgery information leaflets. However, it is accepted the guiding principles used in the construction 
of these booklets may not be universal i.e. another researcher may deem the 'simple' booklet as too detailed or the 'extended' booklet too simple. However, no formal guidelines exist to help establish a distinction between extended and simple information provision, only guidelines for information per se (Audit Commission 1993, Scriven and Tucker 1997, Coulter et al 1998).

The extended information booklet was concerned with Gynaecological Laparoscopic surgery and aimed more at the vigilant coper as such individuals are likely to require more information (Appendix 3). The front cover contained the words 'Information Pack - Laparoscopic Day Surgery' in large bold type. The text within the booklet was divided into two columns (size 12 font) containing subheadings and bullet points. The information followed a logical sequence from pre-operative preparation through to the day of surgery and post-operative recovery. The readability of the extended information booklet was considered utilising a simple but effect method - SMOG Grading System (Ley and Florio 1996). This formula works on the simple assumption that the more words containing three or more syllables, the higher the reading age of the text. The number of words with three or more syllables in the first 30 sentences was calculated (as recommended). This number was 60 for the extended information booklet. The reading age therefore equals the square root of 60 plus $8=15.7$ years. This is slightly higher than recommended as it is suggested that a score of 13 or above may require a college education in order to fully comprehend the leaflet (Mumford 1997). The higher reading age may have occurred as numerous medical terms, central to the text, were employed 
although a simple explanation always followed. However, Coulter (1998) states that "Ironically the insistence on aiming for the lowest possible age as measured by readability formulas may have contributed to the infantile quality of many materials." (p. 226). It is therefore acknowledged that if higher level of information is required, the text may (to some extent) unavoidably gain a higher reading age. The booklet provided more explanations than the simple information booklet i.e. definitions, diagrams, procedural, behavioural and sensory information plus cognitive coping strategies. Additionally, the rationale for care and treatment once discharged from hospital was explained rather than merely provided in instruction format as frequently occurs in many brief information booklets. The extended information booklet was therefore slightly longer than the simple information booklet (Table 8).

TABLE 8

OVERVIEW OF INFORMATION BOOKLETS SENT VIA POST 7 - 10 DAYS PRIOR TO SURGERY

\begin{tabular}{|c|c|c|}
\hline & $\begin{array}{c}\text { EXTENDED } \\
\text { LAPAROSCOPY BOOKLET } \\
\end{array}$ & $\begin{array}{c}\text { SIMPLE } \\
\text { LAPAROSCOPY BOOKLET } \\
\end{array}$ \\
\hline DESIGN & Empowering & Instructional \\
\hline $\begin{array}{l}\text { DEFINITION OF } \\
\text { PROCEDURE }\end{array}$ & Yes & Very brief \\
\hline $\begin{array}{c}\text { EXPLANATION OF TYPES } \\
\text { OF SURGERY }\end{array}$ & Yes & No \\
\hline $\begin{array}{l}\text { PROCEDURAL, BEHAVIOURAL \& } \\
\text { SENSORY INFORMATION }\end{array}$ & Yes & Very brief \\
\hline $\begin{array}{l}\text { NUMBER OF } \\
\text { DIAGRAMS }\end{array}$ & 3 & None \\
\hline COGNITIVE COPING STRATEGIES & Throughout & Very brief \\
\hline $\begin{array}{l}\text { INFORMATION REGARDING } \\
\text { RECOVERY AT HOME }\end{array}$ & $\begin{array}{l}\text { Informative } \\
\text { explanations }\end{array}$ & $\begin{array}{l}\text { Brief and containing } \\
\text { 'do's and don'ts' }\end{array}$ \\
\hline NUMBER OF PAGES & 5 & 3 \\
\hline
\end{tabular}


The simple information booklet was also concerned with Gynaecological Laparoscopic surgery but aimed more at the avoidant coper as such individuals are likely to require less information i.e. the booklet took a much less complex approach (Table 8 p.156) (Appendix 4). Consequently, this information booklet was slightly shorter than the extended information booklet. The front cover contained the words 'Information Pack - Laparoscopic Day Surgery' in large bold type. The text within the booklet was divided into two columns (size 12 font) containing subheadings and bullet points. The information followed a logical sequence from pre-operative preparation through to the day of surgery and post-operative recovery. It was more instructional than the extended information booklet and gave more directive information rather than rationale for care and treatment. Definitions were very brief and the booklet contained little explanation regarding the surgical procedure. Procedural, behavioural and sensory information were also very brief. Cognitive coping strategies were brief but provided throughout. The booklet concluded on an instructional note by stating some 'do's and don'ts' relating to recovery at home.

The readability of the simple booklet was also considered using the above readability formula. The number of words with three or more syllables in the first 30 sentences of the simple information booklet was 55 . The reading age therefore equalled 15.4 years. Again, the readability score for the simple booklet was slightly higher than recommended. However straightforward explanations always followed all medical terms. Although presented here as a basic information leaflet, the resultant simple information booklet contained as much 
information (if not more) than is commonly available to all day surgery patients. Additionally, the information was sent prior to surgery. This was demonstrated in the Literature Review (Chapter 4), to be a very infrequent occurrence.

Secondly, four established questionnaires were utilised in order to help test the stated hypotheses. Each of the four questionnaires was placed together in a pack for practical purposes. The front cover of the questionnaire pack contained a statement suggesting it would take approximately 15 minutes to complete all questionnaires. Also, the statement contained a reminder to bring the questionnaire pack with them on the day of surgery. Each set of questionnaires was placed in a random order within the pack to guard against fatigue bias and order effects. The first page of each questionnaire contained a brief explanation, plus an example in large print concerning the manner in which to respond (Appendices 7 to 10). The questionnaire pack was also single sided, typed in large print and comprised of different coloured paper for each questionnaire (complete pre-operative questionnaire pack utilised in the study - Appendix 13).

The questionnaires were -

$\rightarrow$ Mainz Coping Inventory (Krohne 1989) employed to measure the participants' coping style (Hypotheses 1 to 5 ).

$\rightarrow$ State-Trait Anxiety Inventory (STAI) (Spielberger et al 1983) in shortened form (Marteau and Bekker 1992) employed to measure the participants' State anxiety only (Hypothesis 2).

$\rightarrow$ Multi-dimensional Health Locus of Control (MHLC) (Wallston et al 1978) employed to measure the participants' desired level of control (Hypothesis 4). 
$\rightarrow$ Self-efficacy (Jerusalem and Schwarzer 1992) employed to measure the participants' confidence in their ability to behave in such a way as to produce a desirable outcome (Hypothesis 5).

The first of the established questionnaires, listed above, relates to the Mainz Coping Inventory by Krohne (1989) (Appendix 7). This was designed to ascertain the participants' preferred coping style i.e. vigilant, avoidant, fluctuating (variable information requirements) and flexible coping (adaptable information requirements). Of primary concern here were the extremes of information requirements i.e. vigilant and avoidant coping. Information provision for fluctuating and flexible coping is very difficult to establish. Extended and simple information booklets can be reasonably produced and targeted at vigilant and avoidant copers, respectively. Information booklets for fluctuating and flexible copers would be almost impossible to produce (Table 7 p.138). Flexible copers may accept any information provided and fluctuating copers would have far more obscure information requirements (Diagram 4 p.32). Therefore, fluctuating and flexible coping styles were omitted from the hypotheses. This would obviously result in the discarding of an unknown quantity of data. However, if this study can establish the extremes of coping behaviour then it may be possible to make the assumption that some patients will fall between the two extremes i.e. fluctuating and flexible copers. Recommendations as to the possible level of information required could then be made. However, what must firstly be established is that some day surgery patients do indeed require differing levels of information. 
Four brief scenarios were presented in the Mainz Coping Inventory, each with 10 items relating to the four scenarios. The participant had to answer 'true' or 'false' to each question regarding how they might cope if they found themselves in the circumstances outlined in the scenarios. The scenarios related to i) waiting to be seen by the dentist, ii) walking alone through a city street at night, iii) being the front seat passenger in a car with an inexperienced driver at the wheel and, iv) being a passenger on an aircraft experiencing turbulence. The questionnaire has been validated and utilised in similar studies on numerous occasions (Krohne 1989, Hock et al 1996, Krohne et al 1996). The formula provided was utilised to calculate each participants' vigilant and avoidant coping score, as this is a two-dimensional coping model. Two scores were therefore produced for every participant i.e. vigilant coping score and an avoidant coping score. The group mean vigilant and avoidant scores were then calculated. From the two individual scores, a participants' coping style could then be plotted. The individual vigilant and avoidant scores' relationship with the group mean scores determined the participants' coping style i.e. intersection of the two group means divides the two-dimensional model into four segments (vigilant, avoidant, fluctuating and flexible coping). For example, a high vigilant score and a low avoidant score would determine the person to be a vigilant coper (Diagram 4 p.32).

The second questionnaire was a shortened form of the State-Trait Anxiety Inventory (Spielberger et al 1983). This was utilised to measure State anxiety only as it was a very brief questionnaire (Marteau and Bekker 1992) (Appendix 
8). The shortened form was employed in order to avoid the questionnaire pack becoming too time consuming and possibly leading to fatigue bias (Garden et al 1996). State anxiety was a considerable issue as a mismatch in information provision may produce a highly anxious individual i.e. a vigilant coper might become very anxious when only in receipt of the simple information booklet. Six items required an answer of 'not at all', 'somewhat', 'moderately' or 'very much'. Each of these was given a value of 1, 2, 3 or 4, respectively. The level of State anxiety was determined by totalling the numerical value of the respondent's choice using the provided scoring guide (Marteau and Bekker 1992).

The third questionnaire was the Multi-dimensional Health Locus of Control (MHLC) questionnaire formulated by Wallston et al (1978) (Appendix 9). Two forms currently exist e.g. Form A and B, although the mean scores of Form A and Form B are almost identical. Only Form A was therefore utilised where participants are required to answer 18 items (Wallston et al 1978). Three separate scores for Internal Health Locus of Control, Chance Health Locus of Control and Powerful Others (External Health Locus of Control) were established. Wallston et al (1978) defines these subscales as -

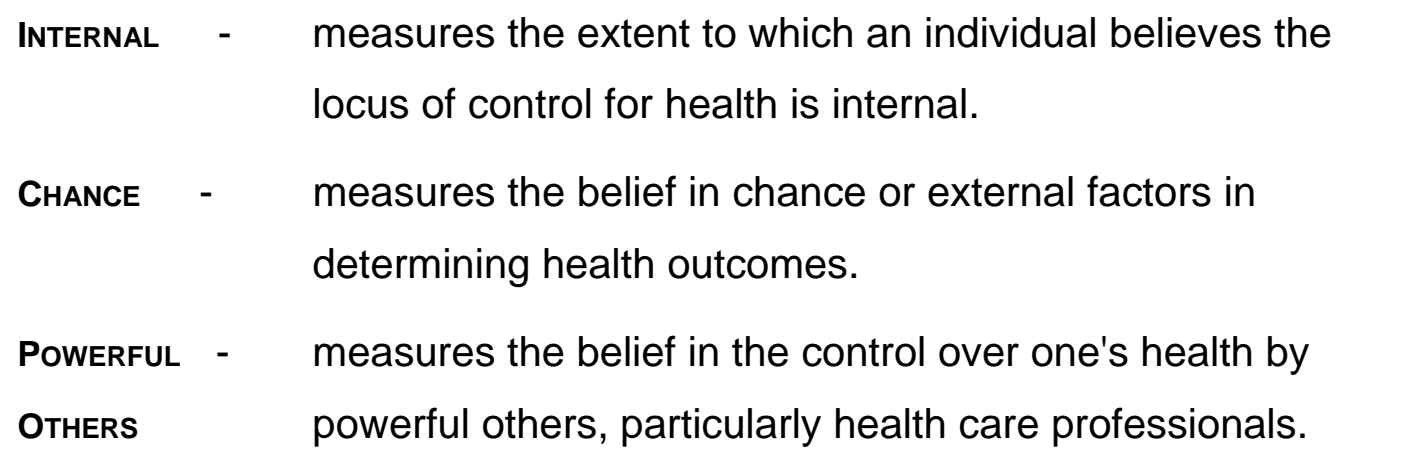


Locus of control has been considered as a 'fixed' aspect of personality by some researchers. However, a number of studies have suggested that health locus of control may not be as rigid as once believed. In an early review of the literature by Strickland (1978) it was suggested the locus of control scale original produced by Rotter (1966) was not always the most appropriate form of measurement in the healthcare situation. Other measures such the Multidimensional Health Locus of Control questionnaire (Wallston et al 1978), may be more accurate predictors of health concerns. Seeman and Seeman (1983) advocated an individual sense of control in the healthcare situation can be selfdetermined or, conversely, a product of the situation i.e. not just reflective of individual desires. Peterson and Stunkard (1989) state "Personal control resides in the transaction between the person and the world; it is neither just a disposition nor a characteristic of the environment." (p. 820). Control was therefore deemed to be context specific and open to manipulation. It is suggested that to obtain a full account of personal health control, the participant should be in the clinical setting or have experience of the clinical setting. Additionally, when in groups, people may possess a 'collective' appraisal of control i.e. group norm. If self-determination in a particular group is perceived as weak (shared belief is of more powerful others), individual perceptions of control may also be influenced in a similar direction (Peterson and Stunkard 1989). Such a shared belief could predominate in an acute hospital day surgery setting where patient stay is minimal and many rules must be followed in order to 
undergo surgery i.e. consent signing, rigid fasting, administration of powerful drugs.

A number of more recent studies have further established that in a healthcare situation, locus of control beliefs may be more susceptible to environmental manipulation, especially the powerful others aspect (Johnston et al 1992, Avis 1994, Halfens 1995) (Chapter 2). In a study by Johnston et al (1992) of 71 patients experiencing rehabilitation, individual health control was observed to be directed by staff and not a spontaneous response by patients. Also, in a study of 22 days surgery patients by Avis (1994), participants perceived themselves as instruments i.e. an object to be worked upon, similar to a car being repaired. Such perceptions forcefully curtailed any individual or group perception of healthcare control. Bartlett (1990) commented that many healthcare professionals are more comfortable with the patient in such a passive, dependent mode and prefer such bureaucratic rules to govern decision-making. Halfens (1995) interviewed 88 surgical patients and concluded health locus of control beliefs changed when an individual was hospitalised, especially the powerful others aspect. "The findings of this study indicate that in new and ambiguous situations, health locus of control can react as a situation-dependent belief." (p. 165). Halfens (1995) further suggested that the powerful others aspect increased on admission and decreased to its original level following discharge.

The modern surgical environment may provide an acute example of how such a shift in external health locus of control appraisal can occur i.e. sparse 
information provision, brief hospital admission for surgery and general anaesthesia within an environment dominated by rigid schedules and enforced by uniformed doctors and nurses (powerful others). The influence of powerful others in uniform in such a situation could considerably enhance such beliefs (outlined above) and overall become a very potent force. Indeed, Milgram (1974) vividly demonstrated how powerful others in uniform could greatly influence perceptions and subsequently shape behaviour. This being the case, vigilant copers who receive a simple level of information (less information than required) in a modern surgical healthcare situation, for example, could be more susceptible to evaluations of increased external health locus of control i.e. powerful others deemed as being more in control of events. In such a situation if a day surgery patient desires an extended level of information and subsequently receives only a simple level, their appraisal of powerful others may be profoundly influenced, albeit on a temporary basis (Seeman and Seeman 1983, Peterson and Stunkard 1989, Halfens 1995).

As participants were asked to complete the questionnaire pack following the reading of the information booklet, a few days prior to surgery, such an influence could be reflected in their responses on their health locus of control questionnaire. Although questionnaire completion occurred prior to the day of surgery i.e. little experience of the influence of powerful others within the day surgery environment, almost all patients had visited the pre-assessment clinic in the weeks and months prior to admission to check physical fitness for surgery. During this brief stay the compelling influence of 'powerful others' in uniform 
would have been vividly demonstrated i.e. told the date and time of their surgery, instructed when to stop eating and drinking, told what time to arrive, instructed what to bring, told what surgery and anaesthesia they will have, told their time of discharge, instructed on how they must get home, instructed on who must look after them and lastly provided with little educational material (61\% of participants in this study received no written information from the day surgery unit or their consultant prior to admission).

The fourth established questionnaire was a Self-efficacy questionnaire by Jerusalem and Schwarzer (1992). This was designed to measure a participant's confidence in their general ability to behave in such a way as to produce a desirable outcome (Appendix 10). Ten items required an answer of 'not at all true', 'barely true', 'moderately true' or 'exactly true'. Each was given a value of $1,2,3$ or 4, respectively. The level of self-efficacy was determined by totalling the numerical value of the respondent's choice. Again, numerous studies have demonstrated how appraisals of self-efficacy can be influenced by external manipulation (Gattuso et al 1992, Cozzarelli 1993, Litt et al 1995). This may be a highly relevant issue within the modern surgical environment as a great deal of preparation and recovery occurs at home.

Finally, the two remaining instruments within this experimental design were the outcome questionnaires i.e. Behavioural Adjustment Rating questionnaire, concerning the nurses' subjective ratings of their patients' adjustment to surgery, and the Participant Satisfaction Rating questionnaire concerning 
participant satisfaction with the information received. The researcher designed both questionnaires.

On the day of surgery the primary nurse involved in the participant's care completed a Behavioural Adjustment Rating questionnaire (Appendix 11). This was primarily concerned with the participant's level of anxiety, their adjustment to day surgery and the number of questions asked regarding their surgery (Hypotheses 2 and 3). It was assumed that a large number of questions asked may indicate a large amount of information was required i.e. they may be a vigilant coper requiring extra information. Nine brief items were included with a choice of four/ five responses. The nurse was required to tick the box adjacent to the most appropriate response.

The scores from items 1 and 9 were used individually to gauge anxiety and adjustment to surgery. Item 1 related to the nurses' ratings of their patient's level of anxiety. A high score was associated with greater anxiety i.e. highly anxious (5), quite anxious (4), uncertain (3), a little anxious (2) and not anxious (1). This score therefore represented the nurses' assessment of patient anxiety - Behavioural Anxiety (Beh_anx). Item 9 related to adjustment to surgery. A high score was associated with good adjustment i.e. good (5), above average (4), average (3), below average (2) and poor (1). This score therefore represented the nurses' assessment of their patients' adjustment to surgery - Behavioural Adjustment (Beh_adjt) (Table 9 p.167). 
TABLE 9

BEHAVIOURAL ADJUSTMENT RATING QUESTIONNAIRE (APPENDIX 11).

\begin{tabular}{|c|c|c|}
\hline \multicolumn{3}{|c|}{ Behavioural Information } \\
\hline $\begin{array}{c}\text { Item } \\
\text { Number }\end{array}$ & Item & Abbreviation \\
\hline 1 & $\begin{array}{l}\text { How anxious would you rate your patient prior her operation } \\
\text { today? }\end{array}$ & Beh_Anx \\
\hline 2 & $\begin{array}{l}\text { Approximately, how much verbal information regarding her } \\
\text { operation and admission to the Day Surgery Unit, did your } \\
\text { patient ask for today? }\end{array}$ & Beh_Info \\
\hline 3 & $\begin{array}{l}\text { Approximately, how much written information regarding her } \\
\text { operation and admission to the Day Surgery Unit, did your } \\
\text { patient ask for today? }\end{array}$ & Beh_Info \\
\hline 4 & $\begin{array}{l}\text { Approximately, how many questions regarding her } \\
\text { operation and admission to the Day Surgery Unit, did your } \\
\text { patient ask today? }\end{array}$ & Beh_Info \\
\hline 5 & $\begin{array}{l}\text { How satisfied did your patient appear to be with the amount } \\
\text { of information you provided before her operation today? }\end{array}$ & Beh_Info \\
\hline 6 & $\begin{array}{l}\text { Approximately, how much did the information you provided } \\
\text { today help to reduce your patient's anxiety? }\end{array}$ & Beh_Info \\
\hline 7 & $\begin{array}{l}\text { As far as you are aware, how many questions did your } \\
\text { patient ask the consultant or surgeon before her operation } \\
\text { today? }\end{array}$ & DISCARDED \\
\hline 8 & $\begin{array}{l}\text { As far as you are aware, how many questions did your } \\
\text { patient ask the anaesthetist before her operation today? }\end{array}$ & DISCARDED \\
\hline 9 & $\begin{array}{l}\text { Overall, how would you rate your patient's adjustment to her } \\
\text { surgery today? }\end{array}$ & Beh_Adjt \\
\hline
\end{tabular}

Items $2,3,4,5,6,7 \& 8$ related to the amount of information observed to be required (Table 9). A numerical score (ranging from 0 to 4 / 6 depending on the number of choices) was placed next to the number of enquires made by the patient. If a large number of questions were asked, a large score was gained and vice versa. This score represented the amount of information the nurses observed the patients (deemed vigilant or avoidant copers) to require Behavioural Information (Beh_Info). Utilising Cronbach's alpha to calculate internal consistency, items $2,3,4,5, \& 6$ gained a level of 0.67 . Although this score is a little lower than desired it does demonstrate some consistency with the items.

Items $7 \& 8$ were completed satisfactorily within the pilot study although not within the final results. The items were to determine how many questions the 
patients asked the surgeon and how many questions the patients asked the anaesthetist. Both items ultimately yielded very little information, as the nurses were frequently not present when the surgeon and/ or anaesthetist spoke to the patient. For item 7 (surgeon), $72.4 \%$ of responses were missing and for item 8 (anaesthetist), $85 \%$ of the responses were missing. As the potential information to be gained from these items was a little tenuous, and poorly reported due mainly to the volume of nursing tasks required during this busy period i.e. immediate pre-operative phase, the results were discarded (Table 9 p.167).

The final questionnaire was the Participant Satisfaction Rating questionnaire. This was also designed by the researcher to ascertain the participants' retrospective view of their experience of day surgery (Appendix 12). The questionnaire was undertaken by telephone interview during the first few postoperative days as it has been established that more accurate and truthful data may be possible once participants have been discharged home (Fitzpatrick and Hopkins 1983, Leinonen et al 1996). The questionnaire was concerned with satisfaction with the information sent by the researcher in the pre-operative period (extended or simple information booklet), satisfaction with the information received overall, satisfaction with the information received on discharge, aspects that helped to reduce anxiety and aspects that increased anxiety (Hypothesis 1 and 2). 
PARTICIPANT SATISFACTION RATING QUESTIONNAIRE (APPENDIX 12).

\begin{tabular}{|c|c|c|}
\hline \multicolumn{3}{|c|}{ Satisfaction with Information Booklet } \\
\hline $\begin{array}{l}\text { Item } \\
\text { Number }\end{array}$ & Item & Abbreviation \\
\hline 1 & $\begin{array}{l}\text { Which of the following did you read before your operation? } \\
\text { (list of materials provided). }\end{array}$ & Info_Req \\
\hline 2 & $\begin{array}{l}\text { How much of the information provided in the study pack did } \\
\text { you read before your operation? }\end{array}$ & Sat_Book \\
\hline 3 & $\begin{array}{l}\text { Were you satisfied with the information you read in the } \\
\text { study pack? }\end{array}$ & Sat_Book \\
\hline 4 & $\begin{array}{l}\text { If you were not very satisfied with the information in the } \\
\text { study pack was it because there was a, great deal less, little } \\
\text { less, little more or great deal more? }\end{array}$ & Sat_Book \\
\hline 5 & $\begin{array}{l}\text { How many times, approximately, did you read the } \\
\text { information provided in the study pack? }\end{array}$ & Sat_Book \\
\hline 6 & $\begin{array}{l}\text { What information did you read or listen to once you were in } \\
\text { the Day Surgery Unit? (list of levels provided). }\end{array}$ & Info_Req \\
\hline 7 & $\begin{array}{l}\text { Were you satisfied with the information you received in the } \\
\text { Day Surgery Unit? }\end{array}$ & Sat_Info \\
\hline 8 & $\begin{array}{l}\text { If you were not very satisfied with the information you } \\
\text { received in the Day Surgery Unit was it because there was? } \\
\text { (choice provided). }\end{array}$ & Info_Req \\
\hline 9 & $\begin{array}{l}\text { Since your operation, have you contacted any of the } \\
\text { following people for help or advice? (list of possible contacts } \\
\text { provided). }\end{array}$ & Info_Req \\
\hline 10 & $\begin{array}{l}\text { Have you read the information provided in the study pack at } \\
\text { home, since your operation? (choice provided). }\end{array}$ & Info_Req \\
\hline 11 & $\begin{array}{l}\text { Ideally, when would you have preferred to receive } \\
\text { information regarding your surgery? (choice provided). }\end{array}$ & Info_Req \\
\hline 12 & $\begin{array}{l}\text { Which of the following people gave you the information you } \\
\text { required about your surgery? (choice provided). }\end{array}$ & Info_Req \\
\hline 13 & $\begin{array}{l}\text { Were you satisfied with the information you received from } \\
\text { these people regarding your surgery? }\end{array}$ & Sat_Info \\
\hline 14 & $\begin{array}{l}\text { If you were not very satisfied with the information you } \\
\text { received from these people regarding your surgery was? } \\
\text { (choice provided). }\end{array}$ & Info_Req \\
\hline 15 & $\begin{array}{l}\text { What method of receiving information would you most } \\
\text { prefer prior to your operation? (choice provided). }\end{array}$ & Info_Req \\
\hline 16 & $\begin{array}{l}\text { What other aspects of your experience of day surgery } \\
\text { helped to reduce your anxiety? }\end{array}$ & ANXIETY DECREASE \\
\hline 17 & $\begin{array}{l}\text { What other aspects of your experience of day surgery } \\
\text { helped to increase your anxiety? }\end{array}$ & ANXIETY INCREASE \\
\hline 18 & $\begin{array}{l}\text { Overall, were you satisfied with the information you } \\
\text { received prior to your operation? }\end{array}$ & Sat_Info \\
\hline
\end{tabular}

Items $7,13 \& 18$ related to the level of satisfaction with the information received overall (Sat_Info) (Table 10). A numerical score (ranging from 0 to 4 I 6 depending on the number of choices) was placed next to each item so that the greater the satisfaction with the information overall, the higher the score and vice versa. This score was totalled and an Overall Satisfaction with Information 
score obtained. Utilising Cronbach's alpha to calculate internal consistency, items $7,13, \& 18$ gained a level of 0.85

Items $1,6,8,9,10,11,12,14 \& 15$ related to the overall level of information required (Table 10 p.169). A numerical score (ranging from 0 to 4 / 6 depending on the number of choices) was placed next to each item so that the greater the level of information required, the higher the score and vice versa. This score was totalled and an overall score regarding Information Requested was obtained (Info_Req). Utilising Cronbach's alpha to calculate internal consistency, items $1,6,8,9,10,11,12,14 \& 15$ gained a level of 0.53 Although clearly lower than desired, information gained prior to admission, educational material from the day surgery unit and information utilised in the discharge period were considered together. Retrospectively, this was not a wise decision and will be commented upon further in the following chapter.

Finally, items 16 and 17 were not numerically scored, as they were to indicate which aspects increased or decreased anxiety on the day of surgery. From this it could be determined what aspects helped to increase and decrease anxiety (Table 10 p.169).

\section{Design}

The main research question was 'What are the effects when two differing levels of information are randomly provided to participants undergoing day surgery who possess a vigilant or avoidant coping style'. The independent variables were the information booklet received and the participants' coping styles. Although coping styles cannot be manipulated they were of consideration as the 
level of information received was manipulated. Additionally, routine admission for day surgery does not take account of the need for differing levels of information. Therefore, such circumstances i.e. consideration of coping style and provision of differing levels of information provision, do not normally prevail. The dependent variables were the level of anxiety, health locus of control appraisal, self-efficacy appraisal, amount of information requested, satisfaction with the information booklet, overall satisfaction with information received plus the nurses' ratings of anxiety, adjustment to surgery and information required.

The independent variables were assembled into a $2 \times 2$ unrelated factorial design in order to measure the effects on the dependent variables (Table 11 p.172). This type of matrix design is very common in experimental research when the independent variables have additional levels. Factorial analysis of variance (ANOVA) was selected to explore the data as each independent variable had two levels or factors i.e. coping style (vigilant and avoidant coping) and information booklet (simple and extended information). Utilising factorial analysis of variance in such a design allows examination of any main experimental effects (influence one factor may have over all levels of another factor) and any experimental interaction effects (influence each factor may have upon the different levels of the second factor). Post-hoc tests were not required as a $2 \times 2$ design was employed i.e. each independent variable only contained two levels. Thereby, any difference observed can only be attributed to one of the two factors under consideration. 
$2 \times 2$ UNRELATED FACTORIAL DESIGN FOR VIGILANT AND AVOIDANT COPING STYLE.

\begin{tabular}{|c|c|c|c|}
\hline & COPING & STYLES \\
\hline & & VIGILANT & AVOIDANT \\
\hline \multirow{2}{*}{$\begin{array}{l}0 \\
u \\
z \\
z\end{array}$} & EXTENDED & $\begin{array}{l}\text { Dependent } \\
\text { variable[s] }\end{array}$ & $\begin{array}{l}\text { Dependent } \\
\text { variable[s] }\end{array}$ \\
\hline & SIMPLE & $\begin{array}{l}\text { Dependent } \\
\text { variable[s] }\end{array}$ & $\begin{array}{l}\text { Dependent } \\
\text { variable[s] }\end{array}$ \\
\hline
\end{tabular}

Essentially, it was predicted that when a mismatch between vigilant and avoidant coping style, and information requirements occurred (independent variables), a negative effect upon the dependent variables would be generated i.e.

- increased state anxiety

- decreased internal health locus of control appraisal and/or

- increased chance health locus of control appraisal and/or

- increased external health locus of control appraisal (powerful others)

- $\quad$ reduced self-efficacy appraisal

- reduced satisfaction with the information booklet

- $\quad$ reduced overall satisfaction with information received

- increased information requested

- increase in anxiety observed by nurses

- $\quad$ poorer adjustment to surgery observed by nurses

- increase in information requirements observed by nurses.

Vigilant and avoidant coping were the only coping styles explored in this study as they are the only two styles whose informational requirements can be confidently predicted. 


\section{Procedure}

Participants' names, addresses and telephone numbers were obtained from a waiting list clerk each week for a period of ten months. This amounted to approximately $\mathrm{n}=250$ potential participants. Contact was attempted by telephoning each participant $7-10$ days prior to the date of surgery. Once contact had been established, the researcher introduced himself as a member of the day surgery staff and the study was briefly outlined. If consent was gained, the inclusion criteria were checked and the participant invited to take part in the study. Information was then sent the following day by first class post. This included an information booklet, covering letter with the researcher's name, address and contact telephone number, consent form and questionnaire pack (Appendices 3 or $4 \& 5$ to 10). This was always 7 to 10 days in advance of surgery as receiving information on the day of surgery has been observed to arrive too late to make any appreciable difference (Young et al 1994). The differing information booklet was the only difference between the materials posted to each participant prior to surgery (Table 12 p.174). Participants who were scheduled to have their surgery on the same day were sent the same information booklet in order to avoid possible contamination of results i.e. ensure independence of participant reporting. Beyond this, allocation of the booklets was an even but random distribution. 


\begin{tabular}{|c|c|}
\hline GROUP ONE & GROUP TWO \\
\hline 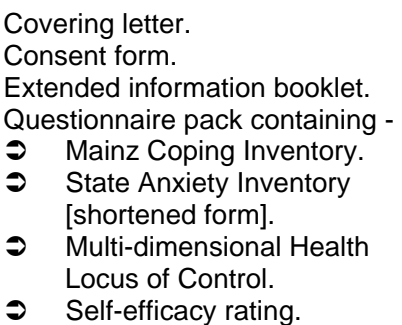 & $\begin{array}{l}\text { Covering letter. } \\
\text { Consent form. } \\
\text { Simple information booklet. } \\
\text { Questionnaire pack containing - } \\
\partial \quad \text { Mainz Coping Inventory. } \\
\partial \quad \text { State Anxiety Inventory } \\
\quad \text { [shortened form]. } \\
\diamond \quad \text { Multi-dimensional Health } \\
\quad \text { Locus of Control. } \\
\diamond \quad \text { Self-efficacy rating. }\end{array}$ \\
\hline
\end{tabular}

Approximately 48 hours prior to surgery all participants were requested (during the initial telephone call and again in the covering letter) to sign the consent form, read the information booklet and complete the questionnaires. They were also asked to bring the completed questionnaire pack and consent form with them on the day of surgery (during the initial telephone call and again in the covering letter). Completing the questionnaires in this way ensured participants' independent responses i.e. they were not influenced by the views of the other participants. Also, all staff in the day surgery unit were completely unaware of which information booklet had been received by whom. Furthermore, at no time were the day surgery staff made aware of the participants' coping styles. The overall plan of the study was therefore initial contact/ gaining consent, information booklet and questionnaires posted 7 to 10 days in advance of surgery, day surgery admission for laparoscopic gynaecological day surgery and finally completion of post-operative questionnaire 2 - 4 days following surgery (Table 13 p.175). 


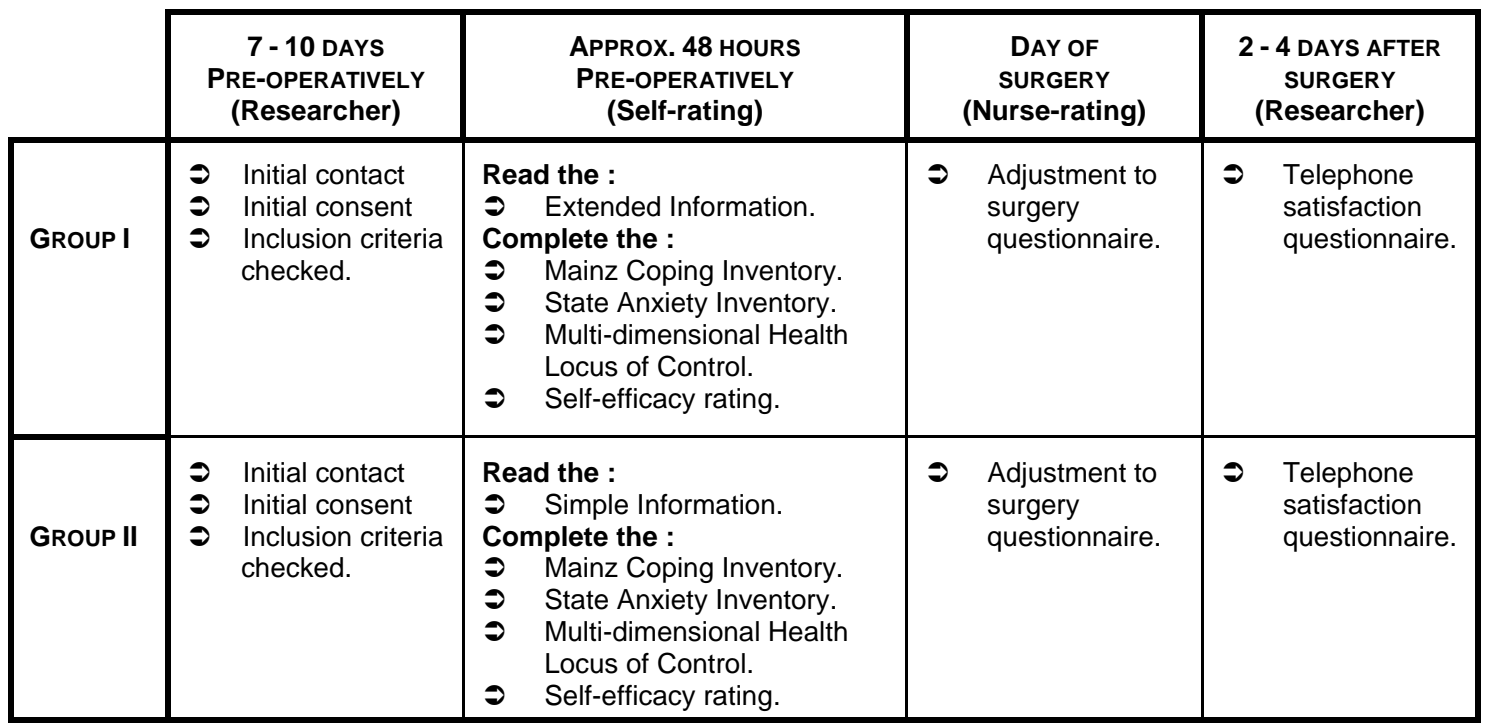

Participants were met for the first and only time by the researcher on the day of surgery in the day surgery unit. The day surgery unit was a well-lit, busy clinical environment, although reasonably quiet. It consisted of a carpeted reception/ waiting area with comfortable chairs, a small ward area with 15 beds/ trolleys and an operating suite situated next to the ward with access via a short corridor. Although the day surgery unit had 15 beds/ trolleys, not all were utilised each day. Commonly there was an operating list each morning and afternoon (Monday to Friday) for the differing surgical specialties. Patients were admitted at either 8.00 am or $1.00 \mathrm{pm}$ on each of these days. All had attended a pre-assessment clinic although this was sometimes many months prior to admission i.e. pre-admission visit to check physical fitness for surgery. The gynaecological surgery operating times varied slightly although Monday, Tuesday and Friday mornings and Wednesday afternoons were the most common times. All the doctors, nurses, theatre and clerical staff within the day 
surgery unit wore uniforms i.e. potent symbols of powerful others. Additionally, the rigid operating schedule was not only very apparent but was referred to in all communications with staff, relatives and patients i.e. the continued enforcement of rules by uniformed powerful others. For example, as the morning operating schedule finished, the next group of patients would be in the reception area waiting for the morning patients to vacate the beds/ trolleys. It would frequently be made clear to the morning patients at the end of the operating schedule that their bed was now required for the next patient waiting in reception.

Shortly after arriving patients were invited into the ward area and allocated a bed/ trolley. The medical and nursing staff then conducted formal admission procedures. This included the nursing staff giving each patient an account of what was to happen, checking they knew what operation they were to undergo and providing answers to any final questions. All the patients were then dressed in a theatre gown and left to wait for their surgery. Although the patients were relative strangers separated by half drawn curtains and bed lockers, some interaction occasionally took place. At this point the patients' companion[s] was invited to wait at the bedside if they so desired. However, this was a very rare occurrence.

Following formal admission procedures, prior to surgery, the researcher conducted the interviews. This normally only took approximately 5 minutes. The interviews were conducted between the hours of $8.30 \mathrm{am}$ and 9.30am and $1.30 \mathrm{pm}$ and $2.30 \mathrm{pm}$ to avoid any disruption to the operating schedule. The 
researcher introduced himself and enquired if the questionnaire pack and consent form had been completed. Once in possession of the completed questionnaire pack and consent form a convenient date and time was arranged in which to conduct a final telephone interview. Brief answers regarding the purpose of the study were repeated when clarification was requested. Each participant was thanked for their co-operation and the Laparoscopy Information booklet (sent to them in the post) left with them for their future use. Participants who attempted to return the information booklet to the researcher (as some did) were advised to keep it and take it home. This was necessary as during the post-operative satisfaction interview participants were asked if they had read the booklet since discharge. At the pre-arranged time, commonly 2 - 4 days following surgery, participants were contacted at home by telephone and the final Participant Satisfaction Rating questionnaire completed (Appendix 12). This final telephone interview took approximately 10 minutes (Table 14 p.178).

While the brief researcher/ participant meeting was taking place on the day of surgery, the primary nurse caring for the participant was requested to complete the Behavioural Adjustment Rating questionnaire. This was concerned with the nurses' observations of the patient's experience of day surgery. The questionnaire took approximately 5 minutes to complete (Appendix 11). Once in possession of both completed questionnaires the nurses were thanked for their co-operation and the research left the unit. 


\begin{tabular}{|c|l|}
\hline CONTACT TIME & \multicolumn{1}{c|}{ RESEARCH ACTIVITY } \\
\hline $\begin{array}{c}\text { Initial contact } \\
\text { (approx. } 7 \text { - } 10 \text { days } \\
\text { prior to surgery) }\end{array}$ & $\begin{array}{l}\text { Each participant contacted by telephone to invite their participation, } \\
\text { briefly outline the study and check inclusion criteria (3 - 5 minutes). }\end{array}$ \\
\hline $\begin{array}{c}\text { Day following initial } \\
\text { contact }\end{array}$ & $\begin{array}{l}\text { Random allocation into two groups. Group 1 to receive the extended } \\
\text { information booklet and Group 2 the simple information booklet. } \\
\text { Information sent by first class post and included - a covering letter } \\
\text { with the researcher's name, address and contact telephone number, } \\
\text { the questionnaire pack, the information booklet and consent form. }\end{array}$ \\
\hline $\begin{array}{c}\text { Approx. 48 hours } \\
\text { prior to surgery }\end{array}$ & $\begin{array}{l}\text { Participants requested to sign the consent form, read the information } \\
\text { booklet, complete the questionnaires and bring the completed } \\
\text { consent form and questionnaire pack with them on the day of } \\
\text { surgery. All staff in the day surgery unit were completely blind as to } \\
\text { which information booklet had been received by whom. }\end{array}$ \\
\hline $\begin{array}{c}\text { Day of surgery } \\
\text { Questionnaire pack retrieved. Convenient date and time arranged in } \\
\text { which to conduct the final telephone interview (5 minutes). Primary } \\
\text { nurse requested to complete questionnaire (2 - 3 minutes). }\end{array}$ \\
\hline $\begin{array}{c}\text { Approx. 2 - 4 days } \\
\text { after surgery }\end{array}$ & Final telephone interview (5 - 10 minutes). \\
\hline
\end{tabular}

\section{Pilot Study}

As with many research studies a pilot study is advisable. A pilot study was therefore employed for approximately the first $10 \%$ of participants. This was to give an indication of how to proceed with the main study. All the participants were happy to take part in the pilot study (and main study) primarily because many had received little information concerning their operation and were therefore willing to take part in order to gain information. However, three problem areas arose and concerned access to waiting lists, initial telephone interviews and the two researcher designed questionnaires i.e. Behavioural Adjustment Rating questionnaire and Participant Satisfaction Rating questionnaire.

Firstly, gaining access to the information regarding who was to be admitted in the subsequent 2 to 3 weeks for surgery was very difficult. Information was 
taken from the day surgery unit's operating lists in the initial stages although these were never completely accurate as the surgeons and medical secretaries would add and subtract names as and when necessary. During the pilot study therefore only 1 or 2 participants were contacted per week using these lists. Consequently many potential participants were lost. A more exact method was required otherwise data collection would exceed the time available. The construction of the operating lists was, however, a very complex task and involved many people i.e. surgeons, medical secretaries, day surgery nurses, day surgery clerical staff and ultimately the patient's compliance (arriving on the arranged day). Eventually a waiting list clerk, who received information from all the above sources and compiled weekly operating lists, was accessed. This person was an extremely important link as she had access to the patients' names, addresses, telephone numbers and dates for surgery. Unfortunately, the researcher was not informed of her role when permission for the study was granted. Only 10 participants had been contacted and all questionnaires completed in the 8 weeks prior to contact with the waiting list clerk. Following contact, a further 9 participants were seen and all questionnaires completed in just ten days!

Secondly, initial contact by telephone was an ongoing problem. Many patients did not leave their telephone number with the hospital (or left an incorrect one), were unable to be contacted via the directory enquiry service, using their address, or simply could not be contacted even though differing days and times were utilised. Initial contact was sometimes a problem as potential 
participants were either unavailable during the day or were too busy caring for their children. The noise from children and the urgency in the participant's voice, as a result of the demands of their children, occasionally made communication too difficult and another more convenient time had to be arranged.

The 'last caller number' or '1471' service recently introduced by British Telecom also created a problem as many potential participants dialled this number upon arriving home to enquire who the researcher was as they had not recognised the telephone number. The researcher made the telephone calls during the day from an office within the University or in the evenings (approximately $6.00 \mathrm{pm}$ until $7.30 \mathrm{pm}$ only) from home. No messages were left on any answerphones during the initial contact, as it was too difficult to fully explain the reason for calling on an answerphone message. Moreover, once day surgery was mentioned, potential participants invariably thought their operation had been cancelled, as this is a common hospital practice prior to admission i.e. telephoning patients a few days or hours prior to admission in order to postpone surgery.

Frequently, on arriving home the potential participants dialled '1471' and upon answering the telephone the researcher often did not immediately know who they were as often, contact with five or six participants had been attempted each evening. An answerphone message was therefore established within the University office and the '141' or the 'caller withheld their number' service always used from the researcher's home in the evening for the initial telephone contact only. This may be considered by some to be somewhat unethical 
although avoiding the anger and distress generated by participants wrongly assuming their operation had been cancelled was deemed more important.

The third a final problem of note concerned an item on each of the researcher designed questionnaires. One of the items within the Behavioural Adjustment Rating questionnaire had to be removed as it asked for the amount of enquiries made by the participants in the post-operative phase, prior to discharge. This was often a very busy period and required the questionnaire to remain within the unit for long periods - sometimes overnight. This became impractical as questionnaires were misplaced and/ or the nurses could not always accurately recall their patient from the previous day. Additionally, it may have been prudent to remove items $7 \& 8$ at this stage i.e. number of questions asked of the surgeon and anaesthetist, as discussed earlier. However, this did not present as a problem during the pilot study. The Participant Satisfaction Rating questionnaire also required a minor adjustment to include 'social arrangements' as an anxiety provoking issue (item 17). Many mothers were concerned about leaving their children all day with relatives or friends while undergoing surgery.

In summary, the waiting list clerk who compiled the gynaecological day surgery operating list was telephoned and visited on a weekly basis to check patient suitability, gain telephone numbers and dates of planned admission. Initial telephone calls continued to be made at varying times of the day until contact was established. If contact was not established within 5 working days of the admission date, the potential participant was discarded. When the initial telephone call was made from the researcher's home, a '141' block was used 
on the telephone to avoid future irrate return callers seeking an explanation. Finally, two items relating to 'Questions asked by the patient in the postoperative phase prior to discharge' were removed from the Behavioural Adjustment Rating questionnaire due to practical purposes. A minor addition was also made to an item on the Patient Satisfaction Rating questionnaire.

\section{Results}

Prior to the presentation of the data, a section describing the participant sample utilised will be briefly outlined. The results relating to each hypothesis will then follow i.e. coping styles, anxiety, adjustment to surgery, health locus of control and self-efficacy. Additionally, within hypothesis one, the distribution of vigilant and avoidant coping scores within the sample will be outlined, as was central to the results. The Statistical Product and Service Solutions computer package (SPSS Version 7) (formally Statistical Product for the Social Sciences) was employed for all analyses. All data were entered and the relevant inferential and descriptive statistics have been employed, where appropriate, within the text to aid explanation. Factorial analysis of variance, commonly used with such research designs, was utilised to examine all data. An acceptable level of statistical significance was set at $p<0.05$.

As will be detailed, only data from $n=51$ participants were employed within the analysis as this was the final number of participants deemed to be either a vigilant or an avoidant coper. Participants deemed as fluctuating and flexible copers were therefore excluded. Additionally, it will be observed through this section, certain abbreviations have been developed to describe each 
independent and dependent variable. Although explanations regarding each variable have been provided throughout the text a complete overview of the abbreviations are provided (Tables $15 \& 16)$.

TABLE 15

ABBREVIATED INDEPENDENT VARIABLES (UTILISED FOR PLOTTING AND ANALYSIS).

\begin{tabular}{|c|c|}
\hline Independent Variables & Abbreviation \\
\hline $\begin{array}{c}\text { Vigilant coping score } \\
\text { (used to calculate coping plot) }\end{array}$ & VigTotal \\
\hline $\begin{array}{c}\text { Avoidant coping score } \\
\text { (used to calculate coping plot) }\end{array}$ & AvoidTot \\
\hline $\begin{array}{c}\text { Coping Plot } \\
\text { (VigTotal \& AvoidTot) }\end{array}$ & CopePlot \\
\hline $\begin{array}{c}\text { Participant coping style } \\
\text { (determined from Coping Plot) }\end{array}$ & Cope_Sty \\
\hline Information type received & Info_Typ \\
\hline
\end{tabular}

TABLE 16

ABBREVIATED DEPENDENT VARIABLES FROM ALL QUESTIONNAIRES (UTILISED FOR ANALYSIS).

\begin{tabular}{|c|c|c|c|}
\hline $\begin{array}{c}\text { Outcomes from } \\
\text { Participants' Ratings }\end{array}$ & $\begin{array}{l}\text { Outcomes from } \\
\text { Nurses' Ratings }\end{array}$ & $\begin{array}{l}\text { Corresponding } \\
\text { Item Number }\end{array}$ & Abbreviation \\
\hline Anxiety & - & - & Anx \\
\hline $\begin{array}{l}\text { Internal health locus } \\
\text { of control }\end{array}$ & - & - & Int_LOC \\
\hline $\begin{array}{c}\text { Chance health locus } \\
\text { of control }\end{array}$ & - & - & Chan_Loc \\
\hline $\begin{array}{l}\text { External health locus } \\
\text { of control }\end{array}$ & - & - & Ext_Loc \\
\hline Self-efficacy & - & - & Self_Eff \\
\hline $\begin{array}{l}\text { Satisfaction with information } \\
\text { booklet }\end{array}$ & - & $2,3,4 \& 5$ & Sat_Book \\
\hline $\begin{array}{l}\text { Overall satisfaction with } \\
\text { information received }\end{array}$ & - & $7,13 \& 18$ & Sat_Info \\
\hline $\begin{array}{c}\text { Amount of information } \\
\text { requested }\end{array}$ & - & $\begin{array}{c}1,6,8,9,10,11,12 \\
14 \& 15\end{array}$ & Info_Req \\
\hline- & $\begin{array}{l}\text { Behavioural rating of } \\
\text { anxiety }\end{array}$ & 1 & Beh_Anx \\
\hline- & $\begin{array}{l}\text { Behavioural rating of } \\
\text { adjustment to surgery }\end{array}$ & 9 & Beh_Adjt \\
\hline- & $\begin{array}{l}\text { Behavioural rating of } \\
\text { information required }\end{array}$ & $2,3,4,5 \& 6$ & Beh_Info \\
\hline
\end{tabular}




\section{Participant Information}

Approximately $\mathrm{n}=250$ patients listed for laparoscopic gynaecological day surgery were extracted from the operating lists over a 10 month period from September 1997 through to May 1998. Many potential participants were unable to be contacted as no answers were gained or incorrect telephone numbers were provided. Therefore, from $n=250$ potential participants, a total of $n=120$ adult day surgery patients undergoing laparoscopic gynaecological surgery and general anaesthesia were invited to take part in the study. However, this figure of $n=120$ was not the final number in the sample. Five participants, for reasons unknown, refused on initial contact to take part once the details of the study were made clear. Following initial consent 11 participants had their operations cancelled, 2 did not attend on the day of surgery, 1 was admitted to hospital as an in-patient prior to surgery, 2 later refused to take part, 1 did not complete one of the questionnaires correctly, 1 participant had to have her operation cancelled on the day of surgery, 9 did not bring the questionnaire pack with them on the day of surgery, 1 complained to her consultant surgeon about the study following consent and was therefore automatically withdrawn and 5 were unable to complete due to researcher ill-health. The final number of participants undergoing laparoscopic gynaecological day surgery who therefore completed all aspects of the study was $n=87$. This gave a response rate of $72.5 \%$. However, only the medical records of $87 \%$ of this number could be accessed ( $n$ $=76)$. From the medical records $n=43$ participants underwent procedural laparoscopic surgery and $n=33$ underwent diagnostic laparoscopic surgery 
(Table 17). This was examined further to reveal the specific laparoscopic gynaecological surgery performed (Table 18).

TABLE 17

LAPAROSCOPIC GYNAECOLOGICAL SURGERY UNDERTAKEN

\begin{tabular}{|ll|r|r|r|r|}
\hline & & & \multicolumn{1}{c|}{$\begin{array}{c}\text { Valid } \\
\text { Percent }\end{array}$} & $\begin{array}{c}\text { Cumulative } \\
\text { Percent }\end{array}$ \\
\hline Valid & Notes unavailable & 11 & 12.6 & 12.6 & 12.6 \\
& Diagnositic & 33 & 37.9 & 37.9 & 50.6 \\
& Procedural & 43 & 49.4 & 49.4 & 100.0 \\
Total & 87 & 100.0 & 100.0 & \\
\hline
\end{tabular}

TABLE 18

SPECIFIC SURGERY UNDERTAKEN

\begin{tabular}{|ll|r|r|r|r|}
\hline & & & Valid \\
Percent & $\begin{array}{c}\text { Cumulative } \\
\text { Percent }\end{array}$ \\
\hline Valid & Medical records unavailable & 11 & 12.6 & 12.6 & 12.6 \\
& Infertility treatment & 17 & 19.5 & 19.5 & 32.2 \\
Sterilisation & 41 & 47.1 & 47.1 & 79.3 \\
Investigations for pain & 13 & 14.9 & 14.9 & 94.3 \\
Investiagtion/ treatment & 4 & 4.6 & 4.6 & 98.9 \\
Sterilisation reversal & 1 & 1.1 & 1.1 & 100.0 \\
Total & 87 & 100.0 & 100.0 & \\
\hline
\end{tabular}

\section{Hypothesis One}

Hypothesis one was concerned with satisfaction with information. The hypothesis stated when an individual with a) an vigilant coping style receives an extended level of pre-operative information, they will be more satisfied with the information than a vigilant coper in receipt of a simple level of pre-operative information, and b) an avoidant coping style receives a simple level of preoperative information, they will be more satisfied with the information than an avoidant coper in receipt of an extended level of pre-operative information.

Utilising the Mainz Coping Inventory, individual coping styles were determined (Krohne 1989). This resulted in participants being categorised as 
vigilant $(n=24)$, avoidant $(n=27)$, fluctuating $(n=23)$ and flexible copers $(n=$ 13) (Diagram 6). Vigilant coping is characterised by the desire for maximum levels of information, avoidant coping by the desire for minimum levels of information, fluctuating coping by the desire for variable levels of information and flexible coping by an adaptable stance regarding information provision.

\section{DIAGRAM 6}

FREQUENCY OF VIGILANT AND AVOIDANT COPING (ALL PARTICIPANTS).

VIGILANT STRATEGIES

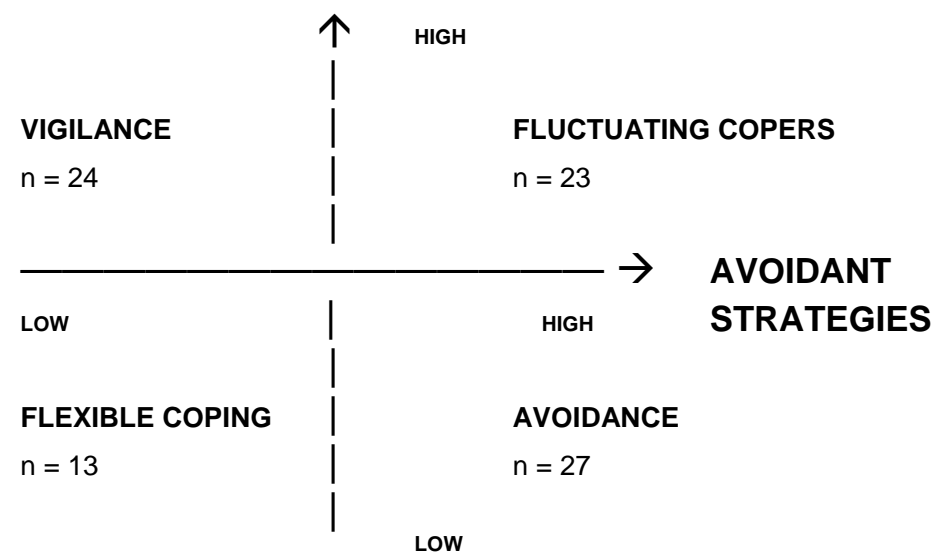

Of the 87 participants, 46 received the extended information booklet and 41 received the simple booklet (Table 19 p.187). The extended information booklet was targeted at vigilant copers who generally prefer an extended level of information. The simple information booklet was targeted at avoidant copers who generally prefer a simple level of information. In addition to receiving the information booklet, $40 \%$ of all participants also received some written information from their hospital consultant or day surgery unit prior to the day of surgery. The level and extent of this information was unknown although such information has traditionally been very simple and brief. Although $40 \%$ of 
participants received written pre-operative information, $100 \%$ of participants stated they desired some written educational material prior to admission.

COPING STYLES POSSESSED BY PARTICIPANTS COMBINED WITH INFORMATION BOOKLET RECEIVED

\begin{tabular}{|c|c|c|}
\cline { 2 - 3 } \multicolumn{1}{c|}{} & $\begin{array}{c}\text { Number Receiving } \\
\text { Extended Information }\end{array}$ & $\begin{array}{c}\text { Number Receiving } \\
\text { Simple Information }\end{array}$ \\
\hline Vigilant copers & 14 & 10 \\
\hline Avoidant copers & 16 & 11 \\
\hline Fluctuating copers & 13 & 10 \\
\hline Flexible copers & 3 & 10 \\
\hline Totals & $\mathbf{4 6}$ & $\mathbf{4 1}$ \\
\hline \multicolumn{2}{r|}{} & $\mathbf{4 6 +} \mathbf{4 1 = 8 7}$ \\
\cline { 2 - 3 } & \multicolumn{2}{c|}{}
\end{tabular}

The distribution of vigilant and avoidant coping scores is central to the study as this determines coping style. As instructed by the Mainz Coping Inventory, two scores were produced for every participant - a vigilant coping score (VigTotal) and an avoidant coping score (AvoidTot) (Krohne 1989). The group mean vigilant and avoidant coping scores were then calculated (Materials section). The vigilant coping mean score was 12.75 and avoidant coping mean score was 11.41. From the two individual scores, a participants' coping style could then be plotted (CopePlot). The individual vigilant and avoidant scores' relationship with the group mean scores determined the participants' coping style i.e. participants deemed vigilant, avoidant, fluctuating or flexible copers (Diagram 6 p.186).

The distribution of the scores for vigilant coping for all $n=87$ participants (VigTotal) was negatively skewed i.e. a tendency for scores to be distributed to the right of the mean. The Kolmogorov-Smirnov test was therefore undertaken 
to test for significance of normality of distribution. The vigilant distribution score was determined not to be significant therefore the sample was normally distributed (Table 20 \& Figure 13).

TABLE 20

DESCRIPTIVE STATISTICS FOR VIGILANT COPING BEHAVIOUR (ALL PARTICIPANTS).

\begin{tabular}{|c|c|c|c|c|c|c|}
\hline & \multicolumn{6}{|c|}{ Cases } \\
\hline & \multicolumn{2}{|c|}{ Valid } & \multicolumn{2}{|c|}{ Missing } & \multicolumn{2}{|c|}{ Total } \\
\hline & $\mathrm{N}$ & Percent & $\mathrm{N}$ & Percent & $\mathrm{N}$ & Percent \\
\hline VIGTOTAL & 87 & $100.0 \%$ & 0 & $.0 \%$ & 87 & $100.0 \%$ \\
\hline
\end{tabular}

\begin{tabular}{|ll|r|r|}
\hline & & \multicolumn{1}{c|}{ Statistic } & \multicolumn{1}{c|}{ Std. Error } \\
\hline VIGTOTAL & Mean & 12.7471 & .3839 \\
& 95\% Confidence & 11.9840 & \\
Interval for Mean & Lower Bound & 13.5102 & \\
& Upper Bound & 12.8429 & \\
5\% Trimmed Mean & 13.0000 & \\
Median & 12.819 & \\
Variance & 3.5804 & \\
Std. Deviation & 1.00 & \\
Minimum & 20.00 & \\
Maximum & 19.00 & \\
Range & 6.0000 & \\
Interquartile Range & -.408 & .258 \\
Skewness & .282 & .511 \\
Kurtosis & & \\
\hline
\end{tabular}

Tests of Normality

\begin{tabular}{|l|c|c|c|}
\hline \multirow{2}{*}{} & \multicolumn{3}{|c|}{ Kolmogorov-Smirnov } \\
\cline { 2 - 4 } & Statistic & df & Sig. \\
\hline VIGTOTAL & .071 & 87 & .200 \\
\hline
\end{tabular}

FIGURE 13

VIGILANT COPING BEHAVIOUR DISTRIBUTION (ALL PARTICIPANTS).

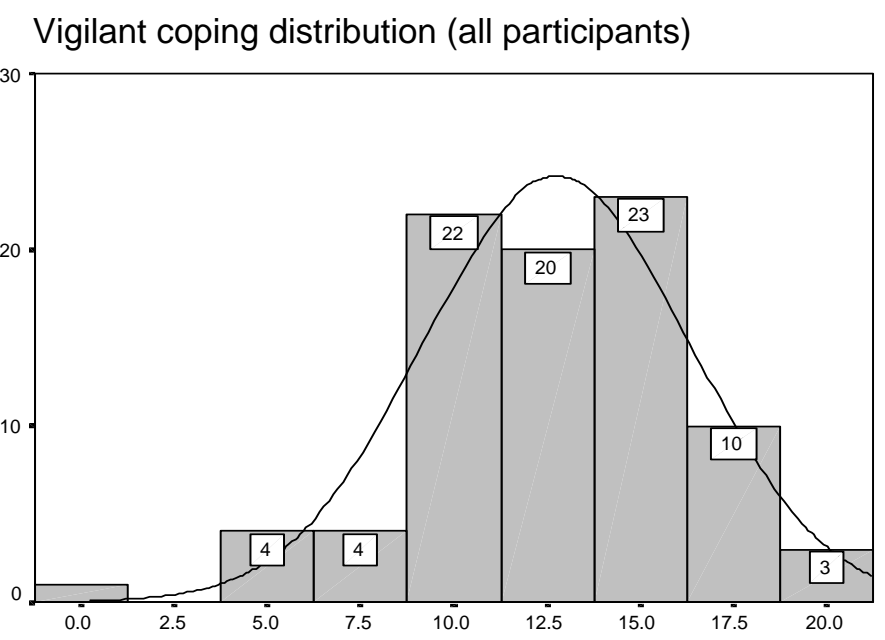


The distribution of the scores for avoidant coping for all $n=87$ participants (AvoidTot), was also negatively skewed. The Kolmogorov-Smirnov test was again undertaken to test for significance of normality of distribution. The avoidant distribution was determined to be significant therefore not normally distributed. This indicated a higher degree of avoidant behaviour in this population sample possibly as a result of the aversive nature of day surgery and general anaesthesia (Table 21 \& Figure 14 p.190). Such a sample with a deviation from normality could influence the analysis although factorial analysis of variance is a robust test with additional steps to check for group variance i.e. Levene's Test.

TABLE 21

DESCRIPTIVE STATISTICS FOR AVOIDANT COPING BEHAVIOUR (ALL PARTICIPANTS).

\begin{tabular}{|c|c|c|c|c|c|c|}
\hline & \multicolumn{6}{|c|}{ Cases } \\
\hline & \multicolumn{2}{|c|}{ Valid } & \multicolumn{2}{|c|}{ Missing } & \multicolumn{2}{|c|}{ Total } \\
\hline & $\mathrm{N}$ & Percent & $\mathrm{N}$ & Percent & $\mathrm{N}$ & Percent \\
\hline AVOIDTOT & 87 & $100.0 \%$ & 0 & $.0 \%$ & 87 & $100.0 \%$ \\
\hline
\end{tabular}

\begin{tabular}{|ll|r|r|}
\hline & & \multicolumn{1}{|c|}{ Statistic } & \multicolumn{1}{|c|}{ Std. Error } \\
\hline AVOIDTOT & Mean & 11.4253 & .3957 \\
95\% Confidence & Lower Bound & 10.6387 & \\
Interval for Mean & Upper Bound & 12.2118 & \\
& & 11.4936 & \\
5\% Trimmed Mean & 12.0000 & \\
Median & 13.619 & \\
Variance & 3.6904 & \\
Std. Deviation & 1.00 & \\
Minimum & 20.00 & \\
Maximum & 19.00 & \\
Range & 5.0000 & \\
Interquartile Range & -.338 & .258 \\
Skewness & .102 & .511 \\
Kurtosis & \multicolumn{2}{c}{} \\
\hline
\end{tabular}

Tests of Normality

\begin{tabular}{|c|c|c|c|}
\hline & \multicolumn{3}{|c|}{ Kolmogorov-Smirnov } \\
\hline & Statistic & $\mathrm{df}$ & Sig. \\
\hline AVOIDTOT & .137 & 87 & .000 \\
\hline
\end{tabular}


AVOIDANT COPING BEHAVIOUR DISTRIBUTION (ALL PARTICIPANTS).

Avoidant coping distribution (all participants)

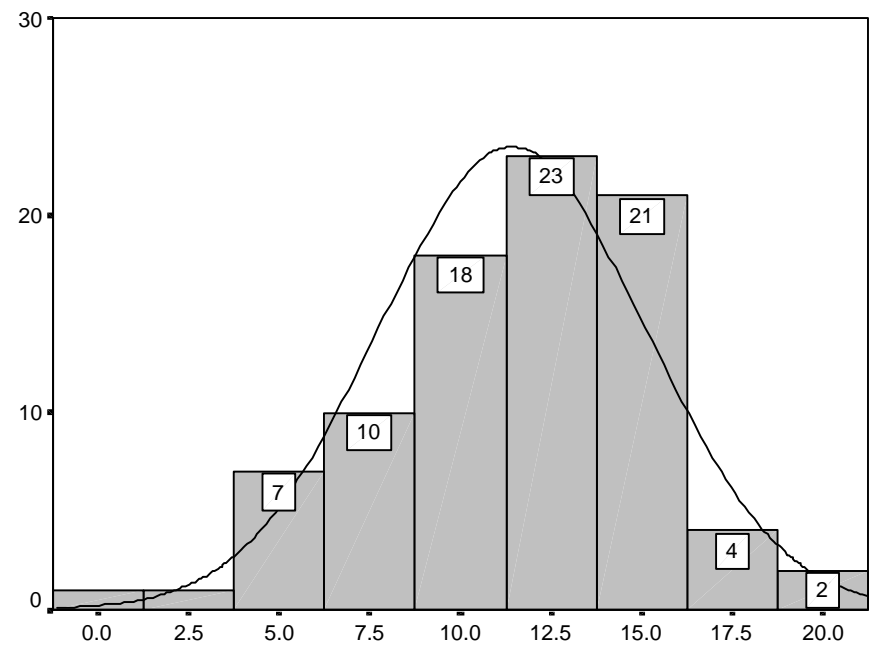

However, only participants deemed as vigilant and avoidant copers were to be utilised. This dictated the use of data from $n=51$ participants i.e. 24 vigilant copers and 27 avoidant copers, where 30 participants received the extended information booklet and 21 received the simple information booklet (Table 22 p.191). The final calculation for these two distinct groups of vigilant and avoidant copers (CopePlot) can be viewed (Figure 15 p.191) (only $n=42$ plots are visible as 9 plots are repeated i.e. overlay occurs). When Figure 15 (p.191) is superimposed over Diagram 6 (p.186) the division of participants into the respective quadrants of the two-dimensional model can be clearly observed. Only utilising approximately $60 \%$ of the original sample in this way resulted in a lower number of participants within each cell of the between subjects factorial design than had been anticipated. Although still a reasonably sized sample on which to conduct statistical analysis, the power of the analysis may have been affected because of this reduction in numbers, as will be highlighted later i.e. 
greater difficulty determining a significant difference between the groups when the sample size in each cell is lower in number (Table 22).

TABLE 22

DISTRIBUTION OF INFORMATION BOOKLETS TO VIGILANT AND AVOIDANT COPERS.

\begin{tabular}{|c|c|c|c|}
\hline & \multicolumn{2}{|c|}{ COPING STYLES } \\
\hline & & V I G I L A N T & $\mathrm{A} \vee \mathrm{O} I \mathrm{DANT}$ \\
\hline \multirow{2}{*}{$\begin{array}{l}0 \\
4 \\
z \\
-\end{array}$} & EXTENDED & 14 & 16 \\
\hline & S I M P LE & 10 & 11 \\
\hline & TOTALS & 24 & 27 \\
\hline & & \multicolumn{2}{|c|}{$24+27=51$} \\
\hline
\end{tabular}

FIGURE 15

VIGILANT AND AVOIDANT COPING PLOT.

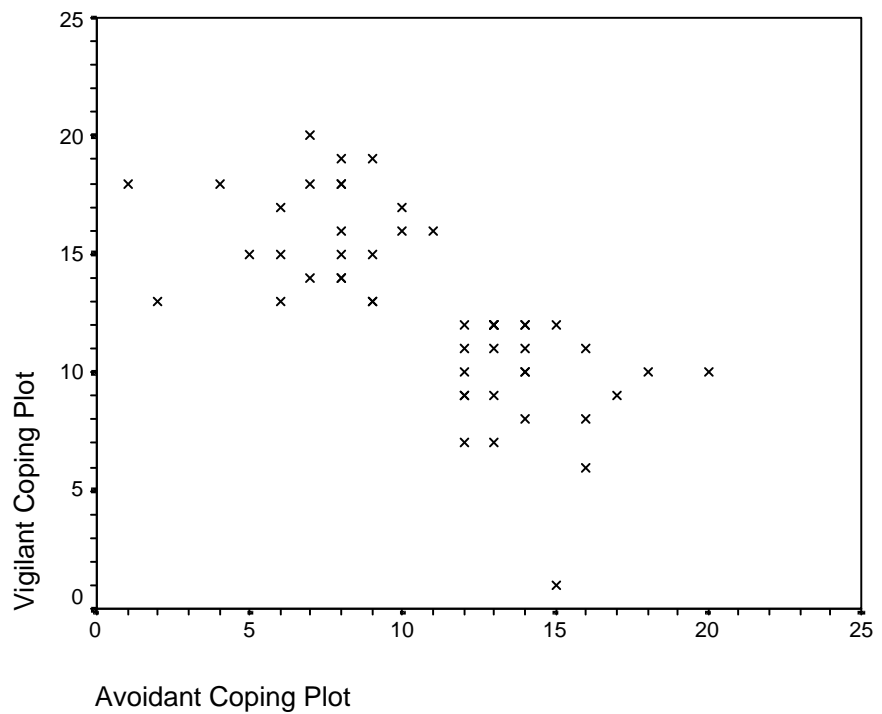

When considering the distribution of scores for vigilant and avoidant coping in this reduced sample of day surgery patients $(n=51)$, vigilant coping behaviour scores (VigTotal) were again negatively skewed. The Kolmogorov-Smirnov test was undertaken to test for significance of normality of distribution. The vigilant distribution score was determined not to be significant and therefore normally distributed (Table 23 p.192 \& Figure 16 p.192). 
TABLE 23

DESCRIPTIVE STATISTICS FOR VIGILANT COPING BEHAVIOUR (VIGILANT COPERS ONLY).

\begin{tabular}{|c|r|r|r|r|r|r|}
\hline \multirow{2}{*}{} & \multicolumn{4}{|c|}{ Cases } \\
\cline { 2 - 8 } & \multicolumn{2}{|c|}{ Valid } & \multicolumn{2}{c|}{ Missing } & \multicolumn{2}{c|}{ Total } \\
\cline { 2 - 8 } & $\mathrm{N}$ & Percent & $\mathrm{N}$ & Percent & $\mathrm{N}$ & Percent \\
\hline VIGILANT & 51 & $58.6 \%$ & 36 & $41.4 \%$ & 87 & $100.0 \%$ \\
\hline
\end{tabular}

\begin{tabular}{|ll|r|r|}
\hline & & \multicolumn{1}{c|}{ Statistic } & Std. Error \\
\hline VIGILANT & Mean & 12.6863 & .5484 \\
95\% Confidence $\quad$ Lower Bound & 11.5847 & \\
Interval for Mean $\quad$ Upper Bound & 13.7878 & \\
& & 12.7821 & \\
& & 12.0000 & \\
Median & 15.340 & \\
Variance & 3.9166 & \\
Std. Deviation & 1.00 & \\
Minimum & 20.00 & \\
Maximum & 19.00 & \\
Range & 6.0000 & \\
Interquartile Range & -.283 & .333 \\
Skewness & .256 & .656 \\
Kurtosis & & \\
\hline
\end{tabular}

Tests of Normality

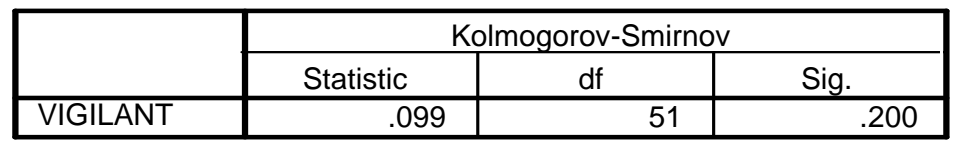

FIGURE 16

VIGILANT COPING BEHAVIOUR DISTRIBUTION (VIGILANT COPERS ONLY).

Vigilant coping scores (final sample)

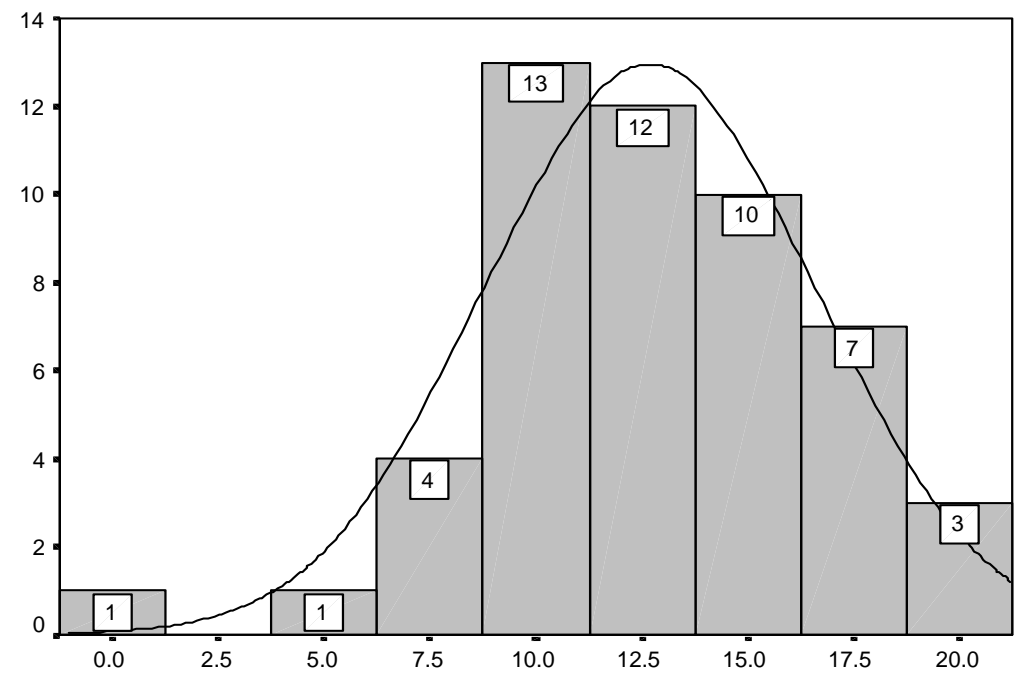


The distribution of scores for avoidant coping in the reduced sample of day surgery patients ( $n=51)$ (AvoidTot) was also negatively skewed with a negative kurtosis i.e. flatter distribution curve or platykurtic. The Kolmogorov-Smirnov test was undertaken to test for significance of normality of distribution. The avoidant distribution was determined to be significant and therefore not normally distributed. Again, the negatively skewed distribution may be indicative of the aversive nature of day surgery and general anaesthesia. However, the weak bimodal distribution and negative kurtosis may have occurred as this reduced sample only encompassed participants with vigilant and avoidant coping behaviours i.e. fluctuating and flexible copers were removed (Table 24 \& Figure 17 p.194). Such a difference may have gained greater transparency here than with the reduced sample of vigilant coping behaviour distribution (Figure 16 p.192), because of the slightly higher number of participants deemed avoidant copers and thereby greater degree of avoidant behaviour.

DESCRIPTIVE STATISTICS FOR AVOIDANT COPING BEHAVIOUR (AVOIDANT COPERS ONLY).

\begin{tabular}{|c|r|r|r|r|r|c|}
\hline \multirow{2}{*}{} & \multicolumn{9}{|c|}{ Cases } \\
\cline { 2 - 8 } & \multicolumn{2}{|c|}{ Valid } & \multicolumn{2}{c|}{ Missing } & \multicolumn{2}{c|}{ Total } \\
\cline { 2 - 8 } & $\mathrm{N}$ & Percent & $\mathrm{N}$ & Percent & $\mathrm{N}$ & Percent \\
\hline AVOIDANT & 51 & $58.6 \%$ & 36 & $41.4 \%$ & 87 & $100.0 \%$ \\
\hline
\end{tabular}




\begin{tabular}{|ll|r|r|}
\hline & & \multicolumn{1}{|c|}{ Statistic } & Std. Error \\
\hline AVOIDANT & Mean & 10.8627 & .5705 \\
& 95\% Confidence $\quad$ Lower Bound & 9.7168 & \\
Interval for Mean $\quad$ Upper Bound & 12.0087 & \\
& & 10.9248 & \\
5\% Trimmed Mean & 12.0000 & \\
Median & 16.601 & \\
Variance & 4.0744 & \\
Std. Deviation & 1.00 & \\
Minimum & 20.00 & \\
Maximum & 19.00 & \\
Range & 6.0000 & \\
Interquartile Range & -.222 & .333 \\
Skewness & -.219 & .656 \\
Kurtosis & & \\
\end{tabular}

Tests of Normality

\begin{tabular}{|c|c|c|c|}
\hline & \multicolumn{3}{|c|}{ Kolmogorov-Smirnov } \\
\hline & Statistic & df & Sig. \\
\hline AVOIDANT & .139 & 51 & .015 \\
\hline
\end{tabular}

FIGURE 17

AVOIDANT COPING BEHAVIOUR DISTRIBUTION (AVOIDANT COPERS ONLY).

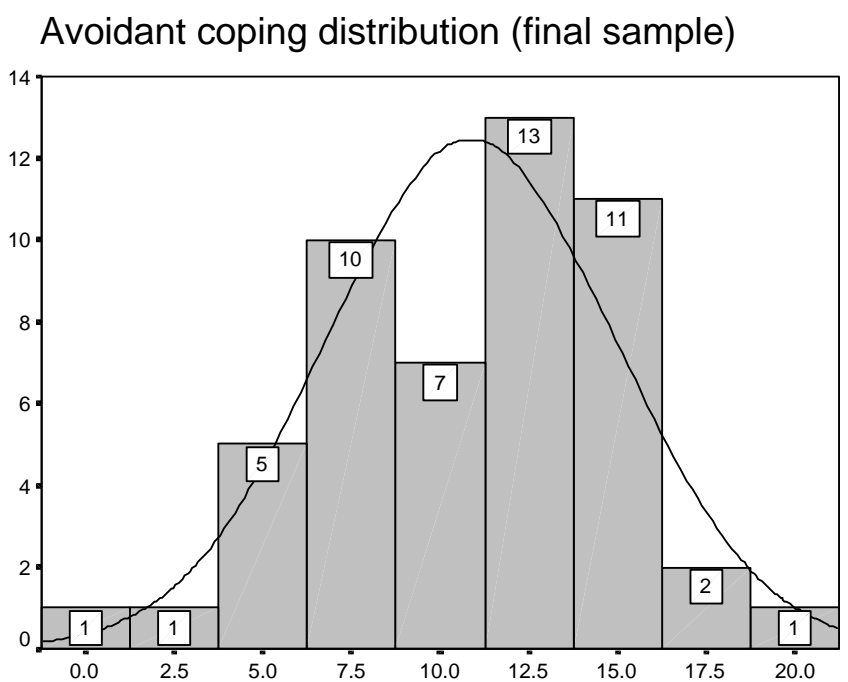

Once participants with a vigilant and avoidant coping style had been clearly identified, testing of the first hypothesis could begin. Firstly, the three self-rated 
outcomes and the nurse rated outcome, pertaining to hypothesis one, were explored utilising factorial analysis of variance.

Satisfaction with information booklet received (Sat_Book) was the first aspect to be considered (Table 25). This was measured using the sum of items $2,3,4$, \& 5 from the Participant Satisfaction Rating questionnaire. These items were constructed to enquired about satisfaction with the information booklet received (Table 10 p.169). There was a non-significant main effect of information booklet received on satisfaction with the booklet $(F(1,47)=0.130, p=0.720)$. This indicates that neither information booklet was deemed to give greater satisfaction than the other.

TABLE 25

SATISFACTION WITH INFORMATION BOOKLET

\begin{tabular}{|ll|l|r|}
\hline & & \multicolumn{1}{|c|}{ Value Label } & N \\
\hline INFO_TYP & 1.00 & Extended & 30 \\
& 2.00 & Simple & 21 \\
COPE_STY & 1.00 & Vigilant Coping & 24 \\
& 2.00 & Avoidant Coping & 27 \\
\hline
\end{tabular}

Dependent Variable: SAT_BOOK

\begin{tabular}{|ll|l|r|r|}
\hline \multirow{2}{*}{ INFO_TYP } & COPE_STY & Mean & $\begin{array}{c}\text { Std. } \\
\text { Deviation }\end{array}$ & $\mathrm{N}$ \\
\hline Extended & Vigilant Coping & 11.9286 & .9972 & 14 \\
& Avoidant Coping & 12.0000 & .8165 & 16 \\
& Total & 11.9667 & .8899 & 30 \\
\hline Simple & Vigilant Coping & 12.0000 & 1.4142 & 10 \\
& Avoidant Coping & 11.7273 & .6467 & 11 \\
& Total & 11.8571 & 1.0623 & 21 \\
\hline Total & Vigilant Coping & 11.9583 & 1.1602 & 24 \\
& Avoidant Coping & 11.8889 & .7511 & 27 \\
& Total & 11.9216 & .9559 & 51 \\
\hline
\end{tabular}




Dependent Variable: SAT_BOOK
\begin{tabular}{|l|r|r|r|r|r|}
\hline & $\begin{array}{c}\text { Type III } \\
\text { Sum of } \\
\text { Squares }\end{array}$ & \multicolumn{1}{c|}{ df } & $\begin{array}{c}\text { Mean } \\
\text { Square }\end{array}$ & \multicolumn{1}{c|}{ F } & Sig. \\
\hline Cource & $.576^{\mathrm{a}}$ & 3 & .192 & .200 & .896 \\
Intercept & 6991.429 & 1 & 6991.429 & 7284.290 & .000 \\
INFO_TYP & .125 & 1 & .125 & .130 & .720 \\
COPE_STY & .125 & 1 & .125 & .130 & .720 \\
INFO_TYP *COPE_STY & .365 & 1 & .365 & .380 & .541 \\
Error & 45.110 & 47 & .960 & & \\
Total & 7294.000 & 51 & & & \\
Corrected Total & 45.686 & 50 & & & \\
\hline
\end{tabular}

a. R Squared $=.013$ (Adjusted R Squared $=-.050$ )

There was also a non-significant main effect of coping style on satisfaction with the information booklet received $(F(1,47)=0.130, p=0.720)$. This indicates that both vigilant and avoidant copers' satisfaction with the information received demonstrated little difference. There was a non-significant interaction effect between information booklet received and coping style, on satisfaction with the information booklet $(F(1,47)=0.380, p=0.541)$. This indicates that satisfaction with the information booklet was not adversely affected when a match between coping style and desired information did not occur (vigilant mean satisfaction with booklet score 11.96 and avoidant mean satisfaction with booklet score 11.89). The lack of significant results here could, in part, have occurred because of a lower level of internal consistency within the researcher designed questionnaire utilised to gather the data (Cronbach's alpha 0.64) (Materials section). Additionally, the power of analysis could have been reduced as a result of the lower number of participants in each cell of the factorial design. 
Secondly, overall satisfaction with the information received was considered (Sat_Info). This was measured using the sum of items $7,13 \& 18$ from the Participant Satisfaction Rating questionnaire. These items were constructed to enquire about satisfaction with information received in total (Table 10 p.169). There was a non-significant main effect of information booklet received on overall satisfaction with information gained $(F(1,47)=0.237, p=0.629)$ (Table 26). This indicates that provision of the extended and simple information booklets demonstrated no appreciable difference on overall satisfaction with information.

\section{TABLE 26}

OVERALL SATISFACTION WITH INFORMATION

Dependent Variable: SAT INFO

\begin{tabular}{|ll|l|r|r|}
\hline \multirow{2}{*}{ INFO_TYP } & COPE_STY & Mean & $\begin{array}{c}\text { Std. } \\
\text { Deviation }\end{array}$ & $\mathrm{N}$ \\
\hline Extended & Vigilant Coping & 18.7857 & 1.6723 & 14 \\
& Avoidant Coping & 18.6875 & 2.4690 & 16 \\
& Total & 18.7333 & 2.0998 & 30 \\
\hline Simple & Vigilant Coping & 18.9000 & 1.3703 & 10 \\
& Avoidant Coping & 18.0000 & 2.3664 & 11 \\
& Total & 18.4286 & 1.9640 & 21 \\
\hline Total & Vigilant Coping & 18.8333 & 1.5228 & 24 \\
& Avoidant Coping & 18.4074 & 2.4061 & 27 \\
& Total & 18.6078 & 2.0306 & 51 \\
\hline
\end{tabular}

Dependent Variable: SAT_INFO

\begin{tabular}{|l|r|r|r|r|r|}
\hline Source & \multicolumn{1}{c|}{$\begin{array}{c}\text { Type III } \\
\text { Sum of } \\
\text { Squares }\end{array}$} & \multicolumn{1}{c|}{ df } & \multicolumn{1}{c|}{$\begin{array}{c}\text { Mean } \\
\text { Square }\end{array}$} & \multicolumn{1}{c|}{ F } & Sig. \\
\hline Corrected Model & $5.462^{\mathrm{a}}$ & 3 & 1.821 & .426 & .735 \\
Intercept & 17028.121 & 1 & 17028.121 & 3987.758 & .000 \\
INFO_TYP & 1.012 & 1 & 1.012 & .237 & .629 \\
COPE_STY & 3.067 & 1 & 3.067 & .718 & .401 \\
INFO_TYP * COPE_STY & 1.979 & 1 & 1.979 & .463 & .499 \\
Error & 200.695 & 47 & 4.270 & & \\
Total & 17865.000 & 51 & & & \\
Corrected Total & 206.157 & 50 & & & \\
\hline
\end{tabular}

a. $\mathrm{R}$ Squared $=.026$ (Adjusted R Squared $=-.036$ ) 
There was also a non-significant main effect of coping style on overall satisfaction with information gained $(F(1,47)=0.718, p=0.401)$. This indicates that coping style i.e. vigilant or avoidant coping, did not influence overall satisfaction with information. There was a non-significant interaction effect between information booklet received and coping style, on overall satisfaction with information $(F(1,47)=0.463, p=0.449)$. This indicates that overall satisfaction with information gained did not increase when the information booklet matched the participants' coping style (vigilant overall mean satisfaction with information score 18.83 and avoidant overall mean satisfaction with information score 18.41). Although the level of internal consistency within the relevant questionnaire items was good (Cronbach's alpha 0.85), the lack of significant results here could, in part, have occurred because the power of analysis may have been reduced.

The third self-rated outcome within hypothesis one concerned the amount of information participants requested (Info_Req). This was measured using the sum of items $1,6,8,9,10,11,12,14 \& 15$ from the Participant Satisfaction Rating questionnaire. These items were constructed to enquire about the requested level of information (Table 10 p.169). There was a non-significant main effect of information booklet received on the requested amount of information $(F(1,47)=0.257, p=0.615)$ (Table 27 p.199). This indicates that the information booklet received did not relate to the amount of information requested e.g. receipt of the simple information booklet did not correspond with a desire for an increased amount of information. 
TABLE 27

REQUESTED AMOUNT OF INFORMATION

Dependent Variable: INFO_REQ

\begin{tabular}{|ll|l|r|r|}
\hline \multirow{2}{*}{ INFO_TYP } & COPE_STY & Mean & $\begin{array}{c}\text { Std. } \\
\text { Deviation }\end{array}$ & \multicolumn{1}{c|}{$\mathrm{N}$} \\
\hline Extended & Vigilant Coping & 37.4286 & 8.2622 & 14 \\
& Avoidant Coping & 38.3125 & 6.3216 & 16 \\
& Total & 37.9000 & 7.1744 & 30 \\
\hline Simple & Vigilant Coping & 37.1000 & 6.6908 & 10 \\
& Avoidant Coping & 36.6364 & 6.1363 & 11 \\
& Total & 36.8571 & 6.2473 & 21 \\
\hline Total & Vigilant Coping & 37.2917 & 7.4919 & 24 \\
& Avoidant Coping & 37.6296 & 6.1840 & 27 \\
& Total & 37.4706 & 6.7627 & 51 \\
\hline
\end{tabular}

Dependent Variable: INFO_REQ

\begin{tabular}{|l|r|r|r|r|r|}
\hline Source & \multicolumn{1}{c|}{$\begin{array}{c}\text { Type III } \\
\text { Sum of } \\
\text { Squares }\end{array}$} & \multicolumn{1}{c|}{ df } & \multicolumn{1}{c|}{$\begin{array}{c}\text { Mean } \\
\text { Square }\end{array}$} & \multicolumn{1}{c|}{ F } & \multicolumn{1}{c|}{ Sig. } \\
\hline Corrected Model & $20.394^{\mathrm{a}}$ & 3 & 6.798 & .141 & .935 \\
Intercept & 68783.599 & 1 & 68783.599 & 1426.472 & .000 \\
INFO_TYP & 12.372 & 1 & 12.372 & .257 & .615 \\
COPE_STY & .544 & 1 & .544 & .011 & .916 \\
INFO_TYP * COPE_STY & 5.590 & 1 & 5.590 & .116 & .735 \\
Error & 2266.312 & 47 & 48.219 & & \\
Total & 73893.000 & 51 & & & \\
Corrected Total & 2286.706 & 50 & & & \\
\hline
\end{tabular}

a. R Squared $=.009$ (Adjusted R Squared $=-.054$ )

There was also a non-significant main effect of coping style on requested amount of information $(F(1,47)=0.11, p=0.916)$ (Table 27). This indicates that the amount of information requested did not produce a match with coping behaviour i.e. vigilant copers did not request a large amount of information and avoidant copers a small amount. There was a non-significant interaction effect between information booklet received and coping style, on requested amount of information $(F(1,47)=0.116, p=0.735)$. This indicates that the requested amount of information was not related to a match between information booklet 
received and coping style (vigilant mean requested amount of information score 37.29 and avoidant mean requested amount of information score 37.63). The lack of significant results here could have occurred for two reasons. Firstly, there was a low level of internal consistency within the researcher-designed questionnaire utilised to gather the data (Cronbach's alpha 0.53) (Materials section). As will be discussed in the next chapter, mixing too many areas of 'satisfaction with information' may have occurred i.e. pre-operative information, day surgery unit information, information received from hospital personnel and post-discharge information. Secondly, the power of analysis could have been reduced because of the lower number of participants in each cell of the factorial design.

The final outcome within hypothesis one concerns the nurses' ratings of information required by patients (Beh_Info). This was measured using the sum of items 2, 3, 4, 5, 6, 7 \& 8 from the Behavioural Adjustment Rating questionnaire. These items were constructed to enquire about the level of information observed to be required (Table 9 p.167). There was a nonsignificant main effect of information booklet received on the nurses' ratings of information required $(F(1,47)=1.439, p=0.236)$ (Table 28 p.201). This indicates that the nurses observed little difference in the demand for information between participants in receipt of the extended and simple information booklets. 
TABLE 28

NURSES' RATINGS OF INFORMATION REQUIRED

Dependent Variable: BEH_INFO

\begin{tabular}{|ll|l|r|r|}
\hline \multirow{2}{*}{ INFO_TYP } & COPE_STY & Mean & $\begin{array}{c}\text { Std. } \\
\text { Deviation }\end{array}$ & \multicolumn{1}{c|}{$\mathrm{N}$} \\
\hline Extended & Vigilant Coping & 10.7143 & 2.7576 & 14 \\
& Avoidant Coping & 10.9375 & 1.7308 & 16 \\
& Total & 10.8333 & 2.2296 & 30 \\
\hline Simple & Vigilant Coping & 11.8000 & 3.9944 & 10 \\
& Avoidant Coping & 11.7273 & 2.5726 & 11 \\
& Total & 11.7619 & 3.2389 & 21 \\
\hline Total & Vigilant Coping & 11.1667 & 3.2925 & 24 \\
& Avoidant Coping & 11.2593 & 2.1048 & 27 \\
& Total & 11.2157 & 2.7005 & 51 \\
\hline
\end{tabular}

Dependent Variable: BEH_INFO

\begin{tabular}{|l|r|r|r|r|r|}
\hline & \multicolumn{1}{c|}{$\begin{array}{c}\text { Type III } \\
\text { Sum of } \\
\text { Squares }\end{array}$} & \multicolumn{1}{c|}{ df } & \multicolumn{1}{c|}{$\begin{array}{c}\text { Mean } \\
\text { Square }\end{array}$} & \multicolumn{1}{c|}{ F } & \multicolumn{1}{c|}{ Sig. } \\
\hline Corrected Model & $11.051^{\mathrm{a}}$ & 3 & 3.684 & .490 & .691 \\
Intercept & 6283.592 & 1 & 6283.592 & 835.262 & .000 \\
INFO_TYP & 10.828 & 1 & 10.828 & 1.439 & .236 \\
COPE_STY & $6.972 \mathrm{E}-02$ & 1 & $6.972 \mathrm{E}-02$ & .009 & .924 \\
INFO_TYP * COPE_STY & .270 & 1 & .270 & .036 & .851 \\
Error & 353.576 & 47 & 7.523 & & \\
Total & 6780.000 & 51 & & & \\
Corrected Total & 364.627 & 50 & & & \\
\hline
\end{tabular}

a. R Squared $=.030$ (Adjusted R Squared $=-.032$ )

There was also a non-significant main effect of coping style on the nurses' ratings of information required $(F(1,47)=0.009, p=0.924)$ (Table 28). This indicates that the nurses observed little difference in demand for information between participants with a vigilant and avoidant coping style. This may, in part, have resulted from the reduced amount of time available prior to surgery for nurse/ patient interaction. There was a non-significant interaction effect between information booklet received and coping style, on nurses' ratings of information required $(F(1,47)=0.036, p=0.851)$. This indicated that the information 
deemed to be required, was not related to a match between information booklet received and coping style e.g. a vigilant coper in receipt of the simple information booklet was not observed to require significantly more information.

Therefore, the claim stating that when a vigilant coper receives an extended level of pre-operative information, they will be more satisfied with the information, than a vigilant coper in receipt of a simple level of pre-operative information, must be rejected and the null hypothesis accepted. Also, the claim stating that when an avoidant coper receives a simple level of pre-operative information, they will be more satisfied with the information than an avoidant coper in receipt of an extended level of pre-operative information, must also be rejected and the null hypothesis accepted.

\section{Hypothesis Two}

Hypothesis two was concerned with anxiety. The hypothesis stated when an individual with a) a vigilant coping style receives an extended level of preoperative information, they will be less anxious than a vigilant coper in receipt of a simple level of pre-operative information, and b) an avoidant coping style receives a simple level of pre-operative information, they will be less anxious than an avoidant coper in receipt of an extended level of pre-operative information.

Two measures were employed to determine participant anxiety i.e. self-rated anxiety and nurse rated anxiety. Firstly, the self-rated state anxiety (Anx) of participants deemed as vigilant and avoidant copers was measured using the shortened form of the State-Trait Anxiety Inventory (Marteau and Bekker 1992). 
However, only the State measure was explored within a factorial analysis of variance test. There was a non-significant main effect of information booklet received on participants' self-rating of anxiety $(F(1,47)=0.040, p=0.843)$ (Table 29). This indicates that the information booklet received demonstrated no appreciable difference on the level of anxiety experienced.

TABLE 29

SELF-RATED STATE ANXIETY

\begin{tabular}{|ll|l|c|}
\hline & & \multicolumn{1}{|c|}{ Value Label } & $\mathrm{N}$ \\
\hline INFO_TYP & 1.00 & Extended & 30 \\
& 2.00 & Simple & 21 \\
COPE_STY & 1.00 & Vigilant Coping & 24 \\
& 2.00 & Avoidant Coping & 27 \\
\hline
\end{tabular}

Dependent Variable: ANX

\begin{tabular}{|ll|l|r|r|}
\hline INFO_TYP & COPE_STY & Mean & $\begin{array}{c}\text { Std. } \\
\text { Deviation }\end{array}$ & \multicolumn{1}{c|}{$\mathrm{N}$} \\
\hline Extended & Vigilant Coping & 12.2857 & 4.0274 & 14 \\
& Avoidant Coping & 11.3125 & 2.8453 & 16 \\
& Total & 11.7667 & 3.4209 & 30 \\
\hline Simple & Vigilant Coping & 13.0000 & 4.8305 & 10 \\
& Avoidant Coping & 10.1818 & 3.0271 & 11 \\
& Total & 11.5238 & 4.1427 & 21 \\
\hline Total & Vigilant Coping & 12.5833 & 4.2927 & 24 \\
& Avoidant Coping & 10.8519 & 2.9182 & 27 \\
& Total & 11.6667 & 3.6968 & 51 \\
\hline
\end{tabular}

Dependent Variable: ANX

\begin{tabular}{|l|r|r|r|r|r|}
\hline & \multicolumn{1}{|c|}{$\begin{array}{c}\text { Type III } \\
\text { Sum of } \\
\text { Squares }\end{array}$} & \multicolumn{1}{c|}{ df } & \multicolumn{1}{c|}{$\begin{array}{c}\text { Mean } \\
\text { Square }\end{array}$} & \multicolumn{1}{c|}{ F } & \multicolumn{1}{c|}{ Sig. } \\
\hline Corrected Model & $49.402^{\mathrm{a}}$ & 3 & 16.467 & 1.221 & .313 \\
Intercept & 6736.816 & 1 & 6736.816 & 499.471 & .000 \\
INFO_TYP & .534 & 1 & .534 & .040 & .843 \\
COPE_STY & 44.252 & 1 & 44.252 & 3.281 & .076 \\
INFO_TYP *COPE_STY & 10.479 & 1 & 10.479 & .777 & .383 \\
Error & 633.931 & 47 & 13.488 & & \\
Total & 7625.000 & 51 & & & \\
Corrected Total & 683.333 & 50 & & & \\
\hline
\end{tabular}

a. $\mathrm{R}$ Squared $=.072$ (Adjusted R Squared $=.013$ ) 
There was also a non-significant main effect of coping style on participants' self-ratings of anxiety $(F(1,47)=3.281, p=0.076)$ (Table 29 p.203). Although the required level of significance was approached $(p=0.05)$ it was not fully gained i.e. vigilant copers' mean anxiety score was greater than the avoidant copers' mean anxiety score. This indicates that there was a tendency (although not significant) for vigilant copers to be more anxious than avoidant copers, irrespective of the information received (Figure 18). There was a non-significant interaction effect between information booklet received and coping style, on participants' self-ratings of anxiety $(F(1,47)=0.777, p=0.383)$. This indicates that self-rated anxiety was not related to a match between the information booklet received and coping style.

FIGURE 18

NON-SIGNIFICANT MAIN EFFECT (COPING STYLE X ANXIETY).

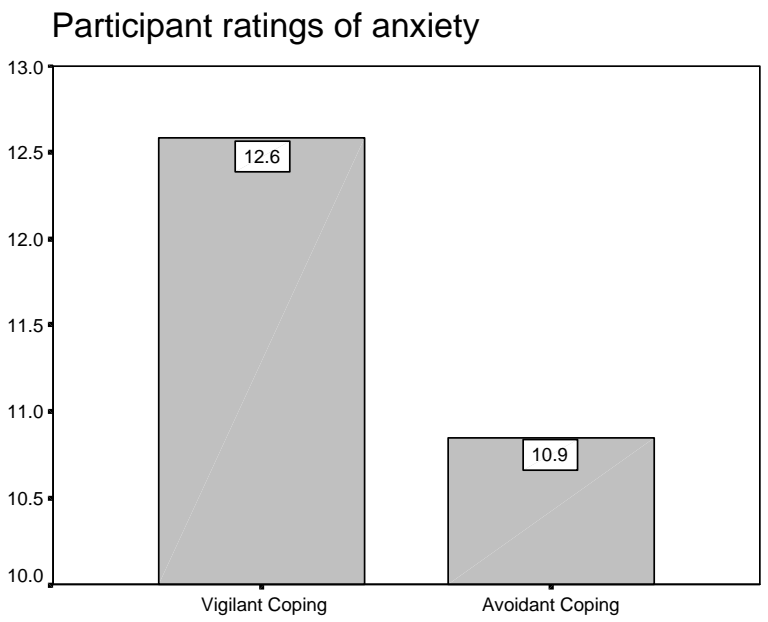

The second measure within hypothesis two concerns the nurses' ratings of anxiety (Beh_Anx) for participants deemed as vigilant and avoidant copers. Behavioural or observed anxiety was measured using item 1 from the Behavioural Adjustment Rating questionnaire. This item was constructed to 
enquire about the level of observed anxiety (Table 9 p.167). Behavioural or observed anxiety was also explored using factorial analysis of variance. There was a significant main effect of information booklet received on the nurses' ratings of anxiety $(F(1,47)=4.257, p=0.045)$ (Table 30). Levene's test demonstrated homogeneity of variance assumptions were not violated, as it was not reported at a significant level i.e. sample scores therefore have similar variance (Table 30). This finding indicates that the participants in receipt of the extended information booklet, irrespective of coping style, were observed to have a lower level of anxiety than those in receipt of the simple information booklet. Additionally, it was the mean vigilant coping score that varied most between receipt of the differing information booklets (2.5 to 3.6$)$ as opposed to the mean avoidant coping score (2.8 to 3.0$)$. Therefore, the mean avoidant coping score varied little between the information booklets received (Table 30 \& Figure 19 p.206).

TABLE 30

NURSE-RATED ANXIETY

Dependent Variable: BEH_ANX

\begin{tabular}{|ll|r|r|r|}
\hline \multirow{2}{*}{ INFO_TYP } & COPE_STY & Mean & $\begin{array}{c}\text { Std. } \\
\text { Deviation }\end{array}$ & \multicolumn{1}{c|}{$\mathrm{N}$} \\
\hline Extended & Vigilant Coping & 2.5000 & .8549 & 14 \\
& Avoidant Coping & 2.8125 & .9811 & 16 \\
& Total & 2.6667 & .9223 & 30 \\
\hline Simple & Vigilant Coping & 3.6000 & 1.2649 & 10 \\
& Avoidant Coping & 3.0000 & 1.3416 & 11 \\
& Total & 3.2857 & 1.3093 & 21 \\
\hline Total & Vigilant Coping & 2.9583 & 1.1602 & 24 \\
& Avoidant Coping & 2.8889 & 1.1209 & 27 \\
& Total & 2.9216 & 1.1286 & 51 \\
\hline
\end{tabular}


Dependent Variable: BEH_ANX

\begin{tabular}{|c|c|c|c|c|c|}
\hline Source & $\begin{array}{l}\text { Type III } \\
\text { Sum of } \\
\text { Squares }\end{array}$ & df & $\begin{array}{l}\text { Mean } \\
\text { Square }\end{array}$ & $\mathrm{F}$ & Sig. \\
\hline Corrected Model & $7.349^{\mathrm{a}}$ & 3 & 2.450 & 2.044 & .121 \\
\hline Intercept & 436.857 & 1 & 436.857 & 364.451 & .000 \\
\hline INFO_TYP & 5.103 & 1 & 5.103 & 4.257 & .045 \\
\hline COPE_STY & .254 & 1 & .254 & .212 & .647 \\
\hline INFO_TYP * COPE_STY & 2.563 & 1 & 2.563 & 2.138 & .150 \\
\hline Error & 56.338 & 47 & 1.199 & & \\
\hline Total & 499.000 & 51 & & & \\
\hline Corrected Total & 63.686 & 50 & & & \\
\hline
\end{tabular}

a. R Squared $=.115$ (Adjusted R Squared $=.059)$

\section{Levene's Test of Equality of Error Variances}

Dependent Variable: BEH_ANX

\begin{tabular}{|c|c|c|c|}
\hline $\mathrm{F}$ & df1 & $\mathrm{df} 2$ & Sig. \\
\hline 1.067 & 3 & 47 & .372 \\
\hline
\end{tabular}

FIGURE 19

SIGNIFICANT MAIN EFFECT (COPING STYLE X NURSE RATED ANXIETY).

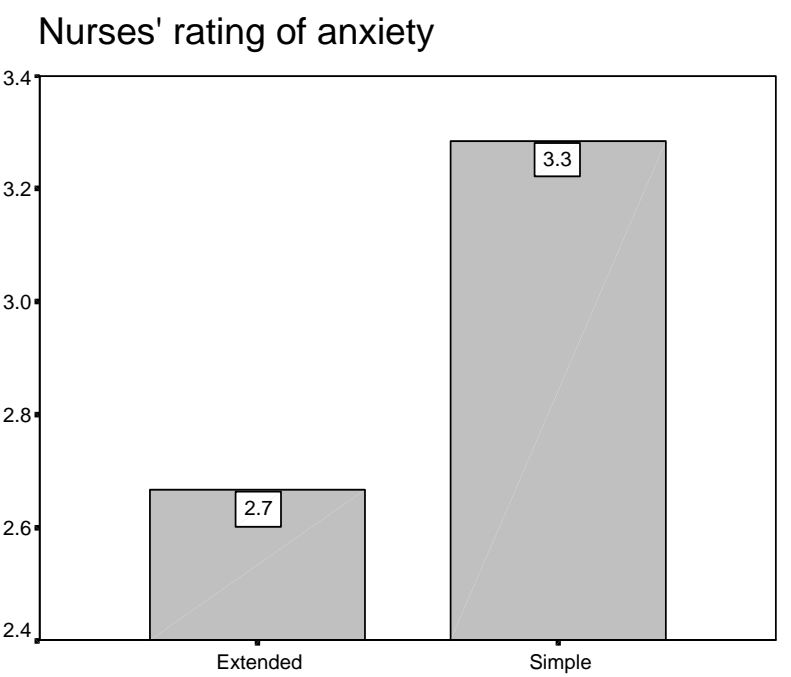

There was a non-significant main effect of coping style on the nurses' ratings of anxiety $(F(1,47)=0.212 p=0.647)($ Table 30 p.205). Again, as with the self-rated anxiety, this indicates that both vigilant and avoidant copers demonstrated no noteworthy difference in anxiety. Some interaction effect 
between information booklet received and coping style, on nurses' ratings of anxiety can be observed (Figure 20). However, this was not at a significant level $(F(1,47)=2.138, p=0.150)$. This indicates the nurses' ratings of anxiety were not related to a match between the information booklet received and coping style i.e. vigilant copers in receipt of the simple information were not viewed as more anxious. However, as stated above, it can be clearly observed that it was the mean vigilant coping score that varied the most between receipt of differing information booklets as opposed to the mean avoidant coping score. Avoidant copers could therefore have gained the information they required from either booklet without adversely influencing their anxiety.

FIGURE 20

NON-SIGNIFICANT INTERACTION EFFECT (COPING STYLE X NURSE RATED ANXIETY).

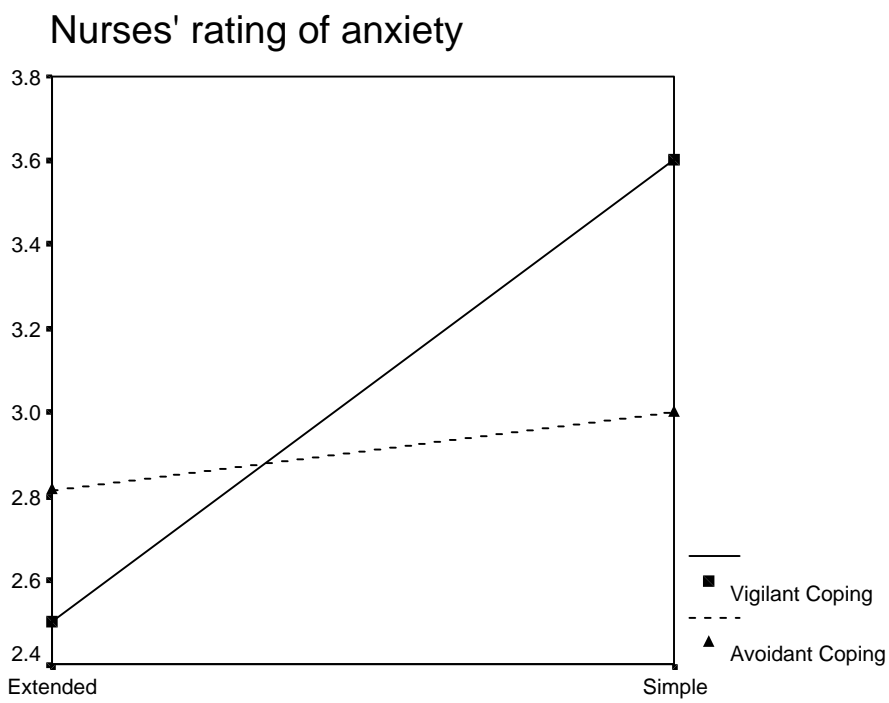

Although it is clear form Figure 20 that some interaction took place in the way predicted (Figure 6 p.144 \& Figure 7 p.145), the mean scores did not provide a significant difference $(p=0.150)$. This may have occurred for two reasons. Firstly, the nurses' brief meeting with their patients may have given rise to a 
limited assessment of anxiety. Secondly, the power of the analysis may have been reduced due to the decreased numbers in each cell of the factorial design.

Therefore, the claim stating that when a vigilant coper, in receipt of an extended level of pre-operative information they will be less anxious than a vigilant coper in receipt of a simple level of pre-operative information, must be rejected and the null hypothesis accepted. Also, the claim stating that when an avoidant coper in receipt of a simple level of pre-operative information, they will be less anxious than an avoidant coper in receipt of an extended level of preoperative information, must also be rejected and the null hypothesis accepted.

\section{Hypothesis Three}

Hypothesis three was concerned with adjustment to surgery. The hypothesis stated when an individual with a) a vigilant coping style receives an extended level of pre-operative information, adjustment to surgery will be more positively influenced in comparison to a vigilant coper in receipt of a simple level of preoperative information, and b) an avoidant coping style receives a simple level of pre-operative information, adjustment to surgery will be more positively influenced in comparison to an avoidant coper in receipt of an extended level of pre-operative information.

Adjustment to surgery for participants deemed as vigilant and avoidant copers was measured using item 9 from the Behavioural Adjustment Rating questionnaire (Beh_Adjt) (Table 9 p.167). This item was constructed to enquire about observed adjustment to surgery and was explored utilising factorial analysis of variance. Firstly, there was a non-significant main effect of 
information booklet received on the nurses' rating of adjustment to surgery (Beh_Adjt) $(F(1,47)=0.008, p=0.928)($ Table 31). This indicates that the information booklet received had little impact upon observed adjustment. There was also a non-significant main effect of coping style on observed adjustment to surgery $(F(1,47)=0.493, p=0.486)$. This indicates that coping style had little influence over observed adjustment to surgery. Finally, there was a nonsignificant interaction effect between information booklet received and coping style, on observed adjustment to surgery $(F(1,47)=0.368, p=0.547)$. This indicates the nurses' assessment of adjustment to surgery was not related to a match between the information booklet received and coping style. Again, however, the lower numbers within each cell of the factorial design may have adversely influenced the power of the analysis and the brief nurse/ patient interaction may have given rise to limited assessment.

TABLE 31

ADJUSTMENT TO SURGERY

\begin{tabular}{|ll|l|c|}
\hline & & \multicolumn{1}{|c|}{ Value Label } & $\mathrm{N}$ \\
\hline INFO_TYP & 1.00 & Extended & 30 \\
& 2.00 & Simple & 21 \\
COPE_STY & 1.00 & Vigilant Coping & 24 \\
& 2.00 & Avoidant Coping & 27 \\
\hline
\end{tabular}

Dependent Variable: BEH_ADJT

\begin{tabular}{|ll|r|r|r|}
\hline \multirow{2}{*}{ INFO_TYP } & COPE_STY & Mean & \multicolumn{1}{c|}{$\begin{array}{c}\text { Std. } \\
\text { Deviation }\end{array}$} & \multicolumn{1}{c|}{$\mathrm{N}$} \\
\hline Extended & Vigilant Coping & 3.5000 & 1.1602 & 14 \\
& Avoidant Coping & 3.8750 & 1.0247 & 16 \\
& Total & 3.7000 & 1.0875 & 30 \\
\hline Simple & Vigilant Coping & 3.7000 & .8233 & 10 \\
& Avoidant Coping & 3.7273 & .9045 & 11 \\
& Total & 3.7143 & .8452 & 21 \\
\hline Total & Vigilant Coping & 3.5833 & 1.0180 & 24 \\
& Avoidant Coping & 3.8148 & .9623 & 27 \\
& Total & 3.7059 & .9858 & 51 \\
\hline
\end{tabular}


Dependent Variable: BEH_ADJT

\begin{tabular}{|l|r|r|r|r|r|}
\hline & $\begin{array}{c}\text { Type III } \\
\text { Sum of } \\
\text { Squares }\end{array}$ & \multicolumn{1}{c|}{ df } & \multicolumn{1}{c|}{$\begin{array}{c}\text { Mean } \\
\text { Square }\end{array}$} & \multicolumn{1}{c|}{ F } & \multicolumn{1}{c|}{ Sig. } \\
\hline Corrected Model & $1.056^{\mathrm{a}}$ & 3 & .352 & .348 & .791 \\
Intercept & 674.513 & 1 & 674.513 & 666.966 & .000 \\
INFO_TYP & $8.412 \mathrm{E}-03$ & 1 & $8.412 \mathrm{E}-03$ & .008 & .928 \\
COPE_STY & .498 & 1 & .498 & .493 & .486 \\
INFO_TYP *COPE_STY & .372 & 1 & .372 & .368 & .547 \\
Error & 47.532 & 47 & 1.011 & & \\
Total & 749.000 & 51 & & & \\
Corrected Total & 48.588 & 50 & & & \\
\hline
\end{tabular}

a. R Squared $=.022$ (Adjusted R Squared $=-.041$ )

Therefore, the claim stating that adjustment to surgery will be more positively influenced for a vigilant coper when in receipt of an extended level of preoperative information in comparison to a vigilant coper in receipt of a simple level of pre-operative information, must be rejected and the null hypothesis accepted. Also, the claim stating adjustment to surgery will be more positively influenced for an avoidant coper in receipt of a simple level of pre-operative information, in comparison to an avoidant coper in receipt of an extended level of pre-operative information, must also be rejected and the null hypothesis accepted.

\section{Hypothesis Four}

Hypothesis four was concerned with health locus of control. The hypothesis stated when an individual with a vigilant or avoidant coping style receives the preferred level of pre-operative information, the desired level of health control will be more readily attained in comparison to a vigilant or avoidant coper in receipt of the non-preferred level of pre-operative information. 
Health locus of control for participants deemed as vigilant copers was measured using the Multi-dimensional Health Locus of Control questionnaire (Wallston et al 1978). The internal, chance and external (powerful others) locus of control data were explored utilising factorial analysis of variance. Firstly, there was a non-significant main effect of information booklet received on participants' self-rating of internal health locus of control (Int_Loc) $(F(1,47)=0.002, p=$ 0.967) (Table 32). This indicates that the information booklet received had little impact upon internal appraisals of health locus of control.

TABLE 32

INTERNAL HEALTH LOCUS OF CONTROL

\begin{tabular}{|ll|l|r|}
\hline & & \multicolumn{1}{|c|}{ Value Label } & N \\
\hline INFO_TYP & 1.00 & Extended & 30 \\
& 2.00 & Simple & 21 \\
COPE_STY & 1.00 & Vigilant Coping & 24 \\
& 2.00 & Avoidant Coping & 27 \\
\hline
\end{tabular}

Dependent Variable: INT_LOC
\begin{tabular}{|ll|r|r|r|}
\hline \multirow{2}{*}{ INFO_TYP } & COPE_STY & Mean & $\begin{array}{c}\text { Std. } \\
\text { Deviation }\end{array}$ & \multicolumn{1}{c|}{$\mathrm{N}$} \\
\hline Extended & Vigilant Coping & 50.7143 & 9.7541 & 14 \\
& Avoidant Coping & 49.8750 & 8.4370 & 16 \\
& Total & 50.2667 & 8.9247 & 30 \\
\hline Simple & Vigilant Coping & 48.0000 & 8.4853 & 10 \\
& Avoidant Coping & 52.3636 & 11.5523 & 11 \\
& Total & 50.2857 & 10.2036 & 21 \\
\hline Total & Vigilant Coping & 49.5833 & 9.1553 & 24 \\
& Avoidant Coping & 50.8889 & 9.6927 & 27 \\
& Total & 50.2745 & 9.3725 & 51 \\
\hline
\end{tabular}

Dependent Variable: INT_LOC

\begin{tabular}{|l|r|r|r|r|r|}
\hline & \multicolumn{1}{c|}{$\begin{array}{c}\text { Type III } \\
\text { Sum of } \\
\text { Squares }\end{array}$} & \multicolumn{1}{c|}{$\mathrm{df}$} & \multicolumn{1}{c|}{$\begin{array}{c}\text { Mean } \\
\text { Square }\end{array}$} & \multicolumn{1}{c|}{$\mathrm{F}$} & \multicolumn{1}{c|}{ Sig. } \\
\hline Corrected Model & $105.004^{\mathrm{a}}$ & 3 & 35.001 & .384 & .765 \\
Intercept & 124314.6 & 1 & 124314.6 & 1362.860 & .000 \\
INFO_TYP & .157 & 1 & .157 & .002 & .967 \\
COPE_STY & 38.238 & 1 & 38.238 & .419 & .520 \\
INFO_TYP * COPE_STY & 83.335 & 1 & 83.335 & .914 & .344 \\
Error & 4287.153 & 47 & 91.216 & & \\
Total & 133296.0 & 51 & & & \\
Corrected Total & 4392.157 & 50 & & & \\
\hline
\end{tabular}

a. $\mathrm{R}$ Squared $=.024$ (Adjusted R Squared $=-.038$ ) 
There was also a non-significant main effect of coping style on participants' self-rating of internal health locus of control $(F(1,47)=0.419, p=0.520)$ (Table 32 p.211). This indicates that coping style had little influence over internal health locus of control appraisals. There was a non-significant interaction effect between information booklet received and coping style, on internal health locus of control appraisal $(F(1,47)=0.914, p=0.344)$. This indicates that participants' internal health locus of control appraisals were not related to a match between the information booklet received and coping style.

Secondly, when examining chance health locus of control appraisals, there was a non-significant main effect of information booklet received on participants' self-ratings of chance health locus of control (Chan_Loc) $(F(1,47)=1.133, p=$ 0.293) (Table 33). This indicates that the information booklet received had little impact upon chance health locus of control appraisals.

TABLE 33

Chance heALTH LOCUS OF CONTROL

Dependent Variable: CHAN_LOC

\begin{tabular}{|ll|l|r|r|}
\hline \multirow{2}{*}{ INFO_TYP } & COPE_STY & Mean & $\begin{array}{c}\text { Std. } \\
\text { Deviation }\end{array}$ & \multicolumn{1}{c|}{$\mathrm{N}$} \\
\hline Extended & Vigilant Coping & 37.8571 & 11.3805 & 14 \\
& Avoidant Coping & 33.0000 & 8.9144 & 16 \\
& Total & 35.2667 & 10.2585 & 30 \\
\hline Simple & Vigilant Coping & 34.8000 & 12.4793 & 10 \\
& Avoidant Coping & 29.2727 & 12.6577 & 11 \\
& Total & 31.9048 & 12.5774 & 21 \\
\hline Total & Vigilant Coping & 36.5833 & 11.6839 & 24 \\
& Avoidant Coping & 31.4815 & 10.5334 & 27 \\
& Total & 33.8824 & 11.2741 & 51 \\
\hline
\end{tabular}


Dependent Variable: CHAN_LOC

\begin{tabular}{|l|r|r|r|r|r|}
\hline & \multicolumn{1}{c|}{$\begin{array}{c}\text { Type III } \\
\text { Sum of } \\
\text { Squares }\end{array}$} & \multicolumn{1}{c|}{ df } & \multicolumn{1}{c|}{$\begin{array}{c}\text { Mean } \\
\text { Square }\end{array}$} & \multicolumn{1}{c|}{ F } & \multicolumn{1}{c|}{ Sig. } \\
\hline Cource & $475.798^{\mathrm{a}}$ & 3 & 158.599 & 1.268 & .296 \\
Intercept & 56046.672 & 1 & 56046.672 & 448.030 & .000 \\
INFO_TYP & 141.696 & 1 & 141.696 & 1.133 & .293 \\
COPE_STY & 331.969 & 1 & 331.969 & 2.654 & .110 \\
INFO_TYP * COPE_STY & 1.382 & 1 & 1.382 & .011 & .917 \\
Error & 5879.496 & 47 & 125.096 & & \\
Total & 64904.000 & 51 & & & \\
Corrected Total & 6355.294 & 50 & & & \\
\hline
\end{tabular}

a. $\mathrm{R}$ Squared $=.075$ (Adjusted R Squared $=.016$ )

Although the main effect of coping style on participants' self-rating of chance health locus of control moved a little towards a significant level $(p=0.05)$, it did not achieve it i.e. there was a tendency for vigilant copers to have increased chance health locus of control appraisals $(F(1,47)=2.654, p=0.110)$ (Table 33 p.212) (Figure 21). This indicates that coping style had little influence over chance health locus of control appraisals. There was a non-significant interaction effect between information booklet received and coping style, on chance health locus of control appraisals $(F(1,47)=0.011, p=0.917)$. This indicates that participants' chance health locus of control appraisals were not related to a match between the information booklet received and coping style.

NON-SIGNIFICANT MAIN EFFECT (COPING STYLE X CHANCE HEALTH LOCUS OF CONTROL).

Chance health locus of control

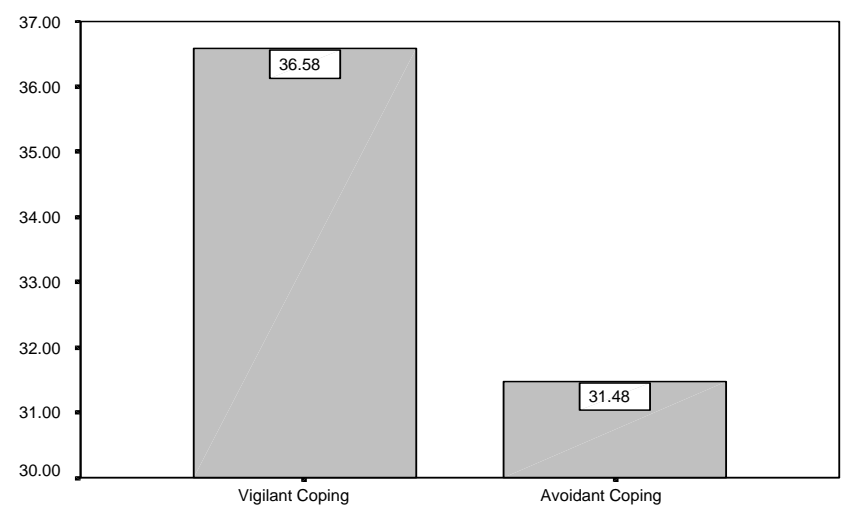


The final aspect within hypothesis four concerns external health locus of control appraisals (Ext_Loc). There was a non-significant main effect of information booklet received on participants' rating of external health locus of control $(F(1,47)=1.105, p=0.298)$ (Table 34). This indicates that the information booklet received had little impact upon external health locus of control appraisals. However, the mean vigilant coping score varied most between receipt of differing information booklets (30.7 to 34.8 ) as opposed to the mean avoidant coping score (25.8 to 27.8$)$. The mean internal health control scores therefore increased for both vigilant and avoidant copers when in receipt of the simple information although much more for the vigilant copers i.e. information type had less of an impact for avoidant copers on internal health control appraisals.

TABLE 34

EXTERNAL HEALTH LOCUS OF CONTROL (POWERFUL OTHERS).

Dependent Variable: EXT_LOC

\begin{tabular}{|ll|l|r|r|}
\hline \multirow{2}{*}{ INFO_TYP } & COPE_STY & Mean & $\begin{array}{c}\text { Std. } \\
\text { Deviation }\end{array}$ & $\mathrm{N}$ \\
\hline Extended & Vigilant Coping & 30.7143 & 11.2758 & 14 \\
& Avoidant Coping & 25.8750 & 10.0524 & 16 \\
& Total & 28.1333 & 10.7374 & 30 \\
\hline Simple & Vigilant Coping & 34.8000 & 9.1019 & 10 \\
& Avoidant Coping & 27.8182 & 9.1850 & 11 \\
& Total & 31.1429 & 9.6036 & 21 \\
\hline Total & Vigilant Coping & 32.4167 & 10.4170 & 24 \\
& Avoidant Coping & 26.6667 & 9.5756 & 27 \\
& Total & 29.3725 & 10.2956 & 51 \\
\hline
\end{tabular}


Dependent Variable: EXT_LOC

\begin{tabular}{|l|r|r|r|r|r|}
\hline & \multicolumn{1}{c|}{$\begin{array}{c}\text { Type III } \\
\text { Sum of } \\
\text { Squares }\end{array}$} & \multicolumn{1}{c|}{ df } & \multicolumn{1}{c|}{$\begin{array}{c}\text { Mean } \\
\text { Square }\end{array}$} & \multicolumn{1}{c|}{ F } & \multicolumn{1}{c|}{ Sig. } \\
\hline Corrected Model & $542.078^{\mathrm{a}}$ & 3 & 180.693 & 1.785 & .163 \\
Intercept & 43746.221 & 1 & 43746.221 & 432.144 & .000 \\
INFO_TYP & 111.895 & 1 & 111.895 & 1.105 & .298 \\
COPE_STY & 430.179 & 1 & 430.179 & 4.249 & .045 \\
INFO_TYP *COPE_STY & 14.132 & 1 & 14.132 & .140 & .710 \\
Error & 4757.844 & 47 & 101.231 & & \\
Total & 49300.000 & 51 & & & \\
Corrected Total & 5299.922 & 50 & & & \\
\hline
\end{tabular}

a. R Squared $=.102($ Adjusted R Squared $=.045)$

Levene's Test of Equality of Error Variances

Dependent Variable: EXT_LOC

\begin{tabular}{|c|c|c|c|}
\hline$F$ & df1 & $\mathrm{df} 2$ & Sig. \\
\hline .293 & 3 & 47 & .830 \\
\hline
\end{tabular}

However, there was a significant main effect of coping style on participants' ratings of external health locus of control $(F(1,47)=4.249, p=0.045)$ (Table 34 p.214). Levene's test demonstrated homogeneity of variance assumptions were not violated, as it was not reported at a significant level. This finding indicates that coping style had an influence over external appraisals of health locus of control i.e. powerful others. As can be observed from the descriptive statistics, vigilant copers experienced greater external health locus of control, irrespective of information received (Table 34 p.214 \& Figure 22 p.216). The impact of 'powerful others' was indeed predicted in such individuals i.e. vigilant copers. However, it was only predicted for vigilant copers in receipt of the simple information booklet. As will be discussed in the next chapter, even the extended information booklet, employed within this study, may have contained too little specific information for the 'information hungry' vigilant coper. 


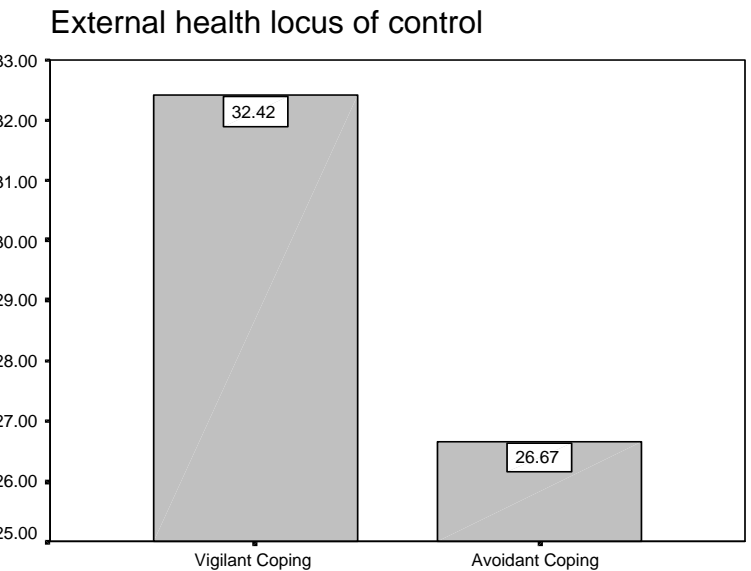

There was a non-significant interaction effect between information booklet received and coping style, on external health locus of control appraisal ( $F(1$, $47)=0.140, p=0.710)$. This indicates that participants' external health locus of control appraisals were not related to a match between the information booklet received and coping style.

Therefore, the claim stating that when an individual with a vigilant or avoidant coping style receives the preferred level of pre-operative information, the desired level of health control will be more readily attained in comparison to a vigilant or avoidant coper in receipt of the non-preferred level of information, must be rejected and the null hypothesis accepted.

\section{Hypothesis Five}

Hypothesis five was concerned with self-efficacy. The hypothesis stated when an individual with a vigilant or avoidant coping style receives the preferred level of pre-operative information, the perceived ability to cope will be more readily 
attained in comparison to a vigilant or avoidant coper in receipt of the nonpreferred level of pre-operative information.

Appraisal of self-efficacy (Self_Eff) by participants deemed as vigilant and avoidant copers was measured using a Self-efficacy questionnaire (Jerusalem and Schwarzer 1992). The self-efficacy data were explored utilising factorial analysis of variance. Firstly, there was a non-significant main effect of information booklet received on participants' rating of self-efficacy $(F(1,47)=$ 0.083, $p=0.775$ ) (Table 35). This indicates that the information booklet received had little impact upon appraisals of self-efficacy. However, there was a significant main effect of coping style on participants' appraisals of self-efficacy $(F(1,47)=6.173, p=0.017)$. Levene's test demonstrated homogeneity of variance assumptions were not violated, as it was not reported at a significant level. This indicates that coping style had a considerable influence over appraisals of self-efficacy. As can be observed from the descriptive statistics, vigilant copers experienced lower self-efficacy appraisals in comparison to avoidant copers, irrespective of information received. However, appraisals were lowest when in receipt of the simple information booklet (Table 35 \& Figure 23 p.218).

TABLE 35

SELF-EFFICACY

\begin{tabular}{|ll|l|c|}
\hline & & Value Label & N \\
\hline INFO_TYP & 1.00 & Extended & 30 \\
& 2.00 & Simple & 21 \\
COPE_STY & 1.00 & Vigilant Coping & 24 \\
& 2.00 & Avoidant Coping & 27 \\
\hline
\end{tabular}


Dependent Variable: SELF_EFF

\begin{tabular}{|ll|r|r|r|}
\hline INFO_TYP & COPE_STY & Mean & $\begin{array}{c}\text { Std. } \\
\text { Deviation }\end{array}$ & \multicolumn{1}{c|}{$\mathrm{N}$} \\
\hline Extended & Vigilant Coping & 30.2143 & 5.4657 & 14 \\
& Avoidant Coping & 32.1875 & 4.5639 & 16 \\
& Total & 31.2667 & 5.0168 & 30 \\
\hline Simple & Vigilant Coping & 28.9000 & 6.2084 & 10 \\
& Avoidant Coping & 34.3636 & 4.9854 & 11 \\
& Total & 31.7619 & 6.1311 & 21 \\
\hline Total & Vigilant Coping & 29.6667 & 5.6926 & 24 \\
& Avoidant Coping & 33.0741 & 4.7711 & 27 \\
& Total & 31.4706 & 5.4492 & 51 \\
\hline
\end{tabular}

Dependent Variable: SELF_EFF

\begin{tabular}{|c|c|c|c|c|c|}
\hline Source & $\begin{array}{l}\text { Type III } \\
\text { Sum of } \\
\text { Squares }\end{array}$ & df & $\begin{array}{c}\text { Mean } \\
\text { Square }\end{array}$ & $F$ & Sig. \\
\hline Corrected Model & $188.466^{\mathrm{a}}$ & 3 & 62.822 & 2.278 & .092 \\
\hline Intercept & 48614.432 & 1 & 48614.432 & 1762.697 & .000 \\
\hline INFO_TYP & 2.287 & 1 & 2.287 & .083 & .775 \\
\hline COPE_STY & 170.260 & 1 & 170.260 & 6.173 & .017 \\
\hline INFO_TYP * COPE_STY & 37.505 & 1 & 37.505 & 1.360 & .249 \\
\hline Error & 1296.240 & 47 & 27.580 & & \\
\hline Total & 51995.000 & 51 & & & \\
\hline Corrected Total & 1484.706 & 50 & & & \\
\hline
\end{tabular}

a. R Squared $=.127$ (Adjusted R Squared $=.071)$

\section{Levene's Test of Equality of Error Variances}

Dependent Variable: SELF_EFF

\begin{tabular}{|c|c|c|c|}
\hline$F$ & df1 & $\mathrm{df} 2$ & Sig. \\
\hline .663 & 3 & 47 & .579 \\
\hline
\end{tabular}

FIGURE 23

SigNIFICANT MAIN EFFECT (COPING STYLE X SELF-EFFICACY).

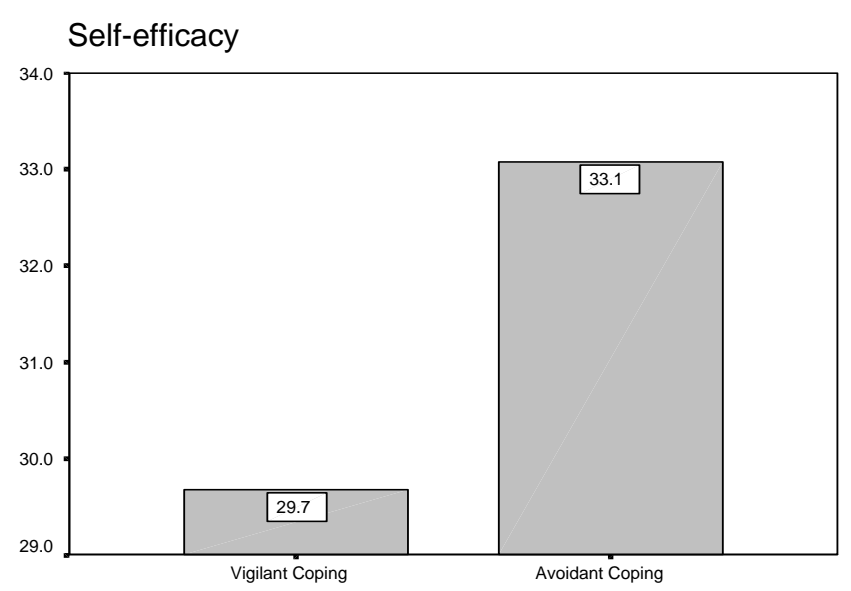


There was a non-significant interaction effect between information booklet received and coping style, on appraisal of self-efficacy $(F(1,47)=1.360, p=$ 0.249). This indicates that participants' appraisals of self-efficacy were not related to a match between the information booklet received and coping style. Lower self-efficacy was predicted for vigilant copers in receipt of the simple information booklet and some interaction indeed took place although not at a significant level (Table 35 p.217). Had the extended information booklet been highly specific (related more to the individual aspects of surgery), the difference for vigilant copers may have been more profound. As will be discussed in the next chapter, the 'general' but extended information booklet utilised within the bounds of this study may have contained too little specific information for a sufficient distinction to be drawn (Figure 24).

FIGURE 24

NON-SIGNIFICANT INTERACTION EFFECT (COPING STYLE X SELF-EFFICACY).

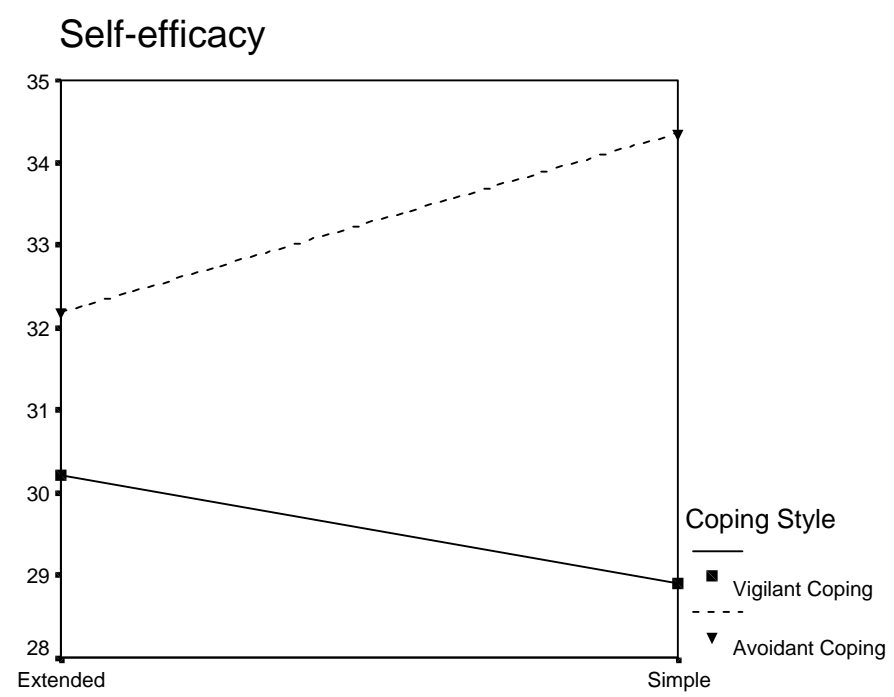

Therefore, the claim stating that when an individual with a vigilant or avoidant coping style receives the preferred level of pre-operative information, the 
perceived ability to cope will be more readily attained in comparison to a vigilant or avoidant coper in receipt of the non-preferred level of information, must be rejected and the null hypothesis accepted.

\section{Summary of Results}

\section{Hypothesis One.}

Hypothesis one stating when an individual with a) an vigilant coping style receives an extended level of pre-operative information, they will be more satisfied with the information than a vigilant coper in receipt of a simple level of pre-operative information, was rejected and the null hypothesis accepted. Part

b) stating when an avoidant coping style receives a simple level of pre-operative information, they will be more satisfied with the information than an avoidant coper in receipt of an extended level of pre-operative information, was also rejected and the null hypothesis accepted.

Firstly, there was a non-significant main effect of information booklet received on satisfaction with information demonstrating that neither booklet was deemed to give greater satisfaction. There was also a non-significant main effect of coping style on satisfaction with information booklet received signifying vigilant and avoidant copers' satisfaction with the information booklet received differed little. There was a non-significant interaction effect between information booklet received and coping style, on satisfaction with the information booklet received. This demonstrated that satisfaction with the information booklet was not adversely affected when a match between coping style and desired information did not occur. 
Secondly, there was a non-significant main effect of information booklet received on overall satisfaction with information gained. This confirmed that provision of the differing booklets offered no appreciable difference on overall satisfaction with information gained. There was also a non-significant main effect of coping style on overall satisfaction with information gained signifying that coping style did not influence overall satisfaction with information. There was a non-significant interaction effect between information booklet received and coping style, on overall satisfaction with information. This established that a match between participants' coping style and preferred level of information did not influence overall satisfaction.

Thirdly, there was a non-significant main effect of information booklet received on the required amount of information. This demonstrated that the information booklet received did not relate to the amount of information required. There was also a non-significant main effect of coping style on required amount of information signifying that the amount of information required did not produce a match with coping style. There was a non-significant interaction effect between information booklet received and coping style, on required amount of information. This confirmed that the required amount of information was not related to a match between information booklet received and coping style.

Finally, there was a non-significant main effect of information booklet received on the nurses' ratings of information required. This established that the nurses observed no difference in demand for information between participants 
in receipt of the differing information booklets. There was also a non-significant main effect of coping style on the nurses' ratings of information required. This signified that the nurses observed no difference in demand for information between participants with a vigilant or avoidant coping style. There was a nonsignificant interaction effect between information booklet received and coping style, on nurses' ratings of information required. This demonstrated that the information deemed to be required was not related to a match between information booklet received and coping style.

\section{Hypothesis Two.}

Hypothesis two stating when an individual with a) a vigilant coping style receives an extended level of pre-operative information, they will be less anxious than a vigilant coper in receipt of a simple level of pre-operative information, was rejected and the null hypothesis accepted. Part b) stating when an avoidant coping style receives a simple level of pre-operative information, they will be less anxious than an avoidant coper in receipt of an extended level of pre-operative information, was also rejected and the null hypothesis accepted.

Firstly, there was a non-significant main effect of information booklet received on participants' self-rating of anxiety. This confirmed the information booklet received established no appreciable difference on level of anxiety. There was also a non-significant main effect of coping style on participants' self-ratings of anxiety signifying that both vigilant and avoidant copers' experience of anxiety was similar $(F(1,47)=3.281, p=0.076)$. Although the required level of 
significance was approached $(p=0.05)$ it was not fully gained i.e. vigilant copers' mean anxiety score was greater than the avoidant copers' mean anxiety score. This indicates that there was a tendency (although not significant) for vigilant copers to be more anxious than avoidant copers, irrespective of the information received. There was a non-significant interaction effect between information booklet received and coping style, on participants' self-ratings of anxiety. This established that anxiety was not related to a match between the information booklet received and coping style.

Secondly, there was a significant main effect of information booklet received on the nurses' ratings of anxiety. This demonstrated that the extended information booklet helped to reduce participant anxiety, irrespective of coping style $(F(1,47)=4.257, p=0.045)$. This finding indicates that the participants in receipt of the extended information booklet, irrespective of coping style, were observed to have a lower level of anxiety than those in receipt of the simple information booklet. There was a non-significant main effect of coping style on the nurses' ratings of anxiety signifying that both vigilant and avoidant copers' experience of anxiety was observed to be similar. Some interaction effect between information booklet received and coping style, on nurses' ratings of anxiety was confirmed although not at a significant level. This indicated that the nurses' ratings of anxiety were not related to a match between the information booklet received and coping style. 


\section{Hypothesis Three.}

Hypothesis three stating when an individual with a) a vigilant coping style receives an extended level of pre-operative information they will demonstrate greater adjustment to surgery than if they had received a simple level of information, was rejected and the null hypothesis accepted. Part b) stating when an avoidant coping style receives a simple level of pre-operative information they will demonstrate greater adjustment to surgery than if they had received an extended level of information, was also rejected and the null hypothesis accepted.

There was a non-significant main effect of information booklet received on the nurses' ratings of adjustment to surgery. This established that the information booklet received had little impact upon observed adjustment to surgery. There was also a non-significant main effect of coping style on observed adjustment to surgery signifying that coping style had little influence over adjustment. Finally, there was a non-significant interaction effect between information booklet received and coping style, on observed adjustment to surgery. This demonstrated that the nurses' assessment of adjustment was not related to a match between the information booklet received and coping style.

\section{Hypothesis Four.}

Hypothesis four stating when an individual with a vigilant or avoidant coping style receives the preferred level of information they will be more satisfied with their perceived level of health locus of control than if they had received the nonpreferred level of information, was rejected and the null hypothesis accepted. 
Firstly, there was a non-significant main effect of information booklet received on participants' self-rating of internal health locus of control. This confirmed that the information booklet received had little impact upon internal health control appraisals. There was also a non-significant main effect of coping style on participants' self-rating of internal health locus of control signifying that coping style had little influence over internal health control appraisals. Some interaction effect between information booklet received and coping style, on internal health locus of control appraisal was established although not at a significant level. This indicated that participants' internal health control appraisals were not related to a match between the information booklet received and coping style.

Secondly, there was a non-significant main effect of information booklet received on participants' self-rating of chance health locus of control. This demonstrated that information booklet received had little impact upon chance health control appraisals. The main effect of coping style on participants' selfrating of chance health locus of control approached a significant level although was not achieved $(F(1,47)=2.654, p=0.110)$. This confirmed that coping style had a tendency to influence chance health control appraisals. There was a non-significant interaction effect between information booklet received and coping style, on chance health locus of control appraisals. This signified that participants' chance health control appraisals were not related to a match between the information booklet received and coping style.

Finally, there was a non-significant main effect of information booklet received on participants' self-rating of external health locus of control. This 
established that information booklet received had little impact upon external health control appraisals. There was a significant main effect of coping style on participants' self-ratings of internal health locus of control signifying that possessing a vigilant coping style had a considerable influence over external health control appraisals, irrespective of information received $(F(1,47)=4.249$, $p=0.045)$. The vigilant coping style therefore exerted an influence over appraisals of powerful others although this was only predicted for vigilant copers in receipt of the simple information booklet and not for all vigilant copers. There was a non-significant interaction effect between information booklet received and coping style, on external locus of control appraisal. This demonstrated that participants' external health control appraisals were not related to a match between the information booklet received and coping style.

\section{Hypothesis Five.}

Hypothesis five stating when an individual with a vigilant or avoidant coping style receives the preferred level of information they will be more satisfied with their perceived ability to cope than if they had received the non-preferred level of information, was rejected and the null hypothesis accepted.

There was a non-significant main effect of information booklet received on participants' rating of self-efficacy demonstrating that the information booklet received had little impact upon self-efficacy appraisals. There was a significant main effect of coping style on participants' appraisals of self-efficacy signifying that appraisal of self-efficacy was considerably lower for participants with a vigilant coping style, irrespective of information booklet received $(F(1,47)=$ 
$6.173, p=0.017)$. The vigilant coping style therefore also exerted an influence over appraisals of self-efficacy although this was only predicted for vigilant copers in receipt of the simple information booklet and not for all vigilant copers. There was a non-significant interaction effect between information booklet received and coping style, on appraisal of self-efficacy. This confirmed that participants' appraisals of self-efficacy were not related to a match between the information booklet received and coping style. 


\section{ChAPTER 6}

\section{Discussion}

The aims of this study were twofold. To discover the most suitable methods of psychologically preparing patients for modern day surgery and to formulate a pre-operative nursing plan which embraced such methods. Only moderate success was gained in achieving these goals as the null hypothesis had to be accepted for each of the stated hypotheses. However, the hypotheses were all concerned with the possible differences within vigilant and avoidant coping style behaviour e.g. a vigilant coper will experience greater anxiety when in receipt of the simple information booklet in comparison to a vigilant coper in receipt of the extended information booklet. A number of pertinent findings arose between the vigilant and avoidant coping style behaviour. Two significant findings were established between coping styles i.e. internal health locus of control and selfefficacy, and one significant finding was related to the information booklets i.e. information booklet received and observed anxiety. Two additional findings were established again between coping styles although these marginally failed to reach the required level of statistical significance i.e. self-rated anxiety and chance health locus of control.

In order to detail these findings, the results from each hypothesis will be discussed in numerical order i.e. information provision, patient anxiety, adjustment to surgery, health locus of control and self-efficacy. This will be followed by suggestions for future research in order to build upon these findings. Finally, an overview of the limitations of the study will be outlined. 


\section{Information Provision}

Hypothesis one a) predicting when an individual with a vigilant coping style receives an extended level of pre-operative information, they will be more satisfied with the information than a vigilant coper in receipt of a simple level of pre-operative information, was rejected and the null hypothesis accepted. Hypothesis one b) predicting when an individual with an avoidant coping style receives a simple level of pre-operative information, they will be more satisfied with the information than an avoidant coper in receipt of an extended level of pre-operative information, was also rejected and the null hypothesis accepted.

Three aspects will be discussed regarding hypothesis one i.e. sample size, construction of materials (researcher designed questionnaires and information booklets) and participants' coping styles. Firstly, the utilisation of a larger sample than the one employed $(n=87)$ may have helped to produce the results predicted in the above hypothesis. Almost one-third of the sample had to be discarded as a number of participants were deemed fluctuating and flexible copers i.e. having more unpredictable information requirements. This led to the use of a much smaller sample $(n=51)$ than had been originally anticipated and consequently a reduction in the power of the statistical analysis i.e. smaller number in each cell of the factorial design. Other studies, although gaining the predicted interaction in hypothesis one, have likewise encountered a lack of the required level of significance due to an insufficient sample size (Gattuso et al 1992). 
In order to compensate for this inevitable loss of participants, more recent studies have employed a far larger sample i.e. 392 participants (Krohne et al 2000). Such a sample size would be highly effective in gaining a substantial number of participants with a vigilant and avoidant coping style although would require a considerable amount of time in which to collect data. Other studies have adopted a different approach selecting only to include from the outset, participants with a vigilant and avoidant coping style, from a larger original sample. Miller and Mangan (1983) engaged 20 vigilant copers and 20 avoidant copers from a larger sample although the number from which this final figure of participants was extracted is unreported. Kohlmann et al (1996) also utilised 40 participants who were either vigilant or avoidant copers from an original sample of 254 participants.

The second issue for hypothesis one concerns the construction of the materials i.e. the two researcher designed questionnaires and information booklets. The way in which the researcher-designed questionnaires were constructed may have limited some of the findings concerning satisfaction with information. Within the Participant Satisfaction Rating questionnaire the internal consistency was low on one particular outcome i.e. Information Requested (Info_Req). Therefore, the measure being pursued was not truly represented by this outcome. Within this outcome, a range of items were employed to determine the various avenues a participant might follow in order to gain the necessary information. However, the items may have proven to be too varied. A more limited approach may have gathered somewhat restricted, although more 
accurate data. For example, the items attempted to determine the amount of pre-operative information read, information gained while in the day surgery unit, information received from hospital personnel, most preferred time to receive information and the amount of post-discharge information read (Table 10 p.169). Although tracking varied lines of enquiry in order to encapsulate this elusive measure, the overall purpose was to determine the amount of information pursued e.g. a vigilant coper might search and exhaust all avenues of enquiry. In order to avoid this awkward problem of attempting to scrutinise the many avenues of information explored by participants, a more recent study concerning coping styles and surgery has validated an instrument which has only three items regarding the amount of information requested i.e. information gained by self (immediate and long-term care) and information gained from the medical staff (Krohne et al 2000).

The Behavioural Adjustment Rating questionnaire, although only brief, may have encountered a number of difficulties i.e. inaccurate reporting due to limited nurse/ patient contact and lack of time to complete the questionnaire. The reduced amount of contact between nurse and patient prior to surgery may have limited accurate reporting of the psychological impact of day surgery. The physical attention required by the rapid admission and discharge of day surgery patients may have, in some instances, impacted upon the quantity and quality of nurse/ patient interaction i.e. assessment of emotional status may not always have been optimal as a result of other essential medical priorities (Spitzer 1998, Leinonen et al 2001). 
The responsibility for undertaking essential medical care together with the rapid admission and discharge of patients may likewise have influenced the time necessary to accurately complete the Behavioural Adjustment Rating questionnaire. Indeed, two essential items from this questionnaire had to be discarded due to under-reporting i.e. the amount of participants' questions directed at the surgeon and the amount directed at the anaesthetist (Table 9 p.167). This under-reporting occurred, as $85 \%$ of nurses were not present when participants were conversing with the anaesthetist and $72.4 \%$ were not present when participants were conversing with the surgeon. Such data could have proven invaluable in gauging the level of information required by participants. Numerous studies concerning the psychological impact of surgery have consistently identified anaesthesia and proposed surgery as two main causes of pre-operative anxiety (Egbert et al 1964, Ramsay 1972, Male 1981, McCleane and Cooper 1990, Mitchell 1997, McGaw and Hanna 1998). Therefore, during the disclosure of information regarding two well-documented stressful events i.e. anaesthesia and proposed surgery, the majority of nurses were busy elsewhere. If the improved pre-operative psycho-educational care of day surgery patients is an ultimate goal, such challenges in modern surgery must be confronted.

The construction and utilisation of the Laparoscopic Information Booklets also presented a number of challenges. For the avoidant coper, both the simple and extended information booklets may have provided the required level of information (Patient Anxiety section). This may have negated any attempt to 
explore the differences in the information requirements of avoidant copers. Indeed, as a mismatch between coping style and information provision was central to all hypotheses, this may have contributed to the overall lack of significant outcomes concerning all avoidant coping behaviour. In addition, the extended information booklet may have provided too little information for the vigilant coper. The Laparoscopic Information Booklets contained 'general' information pertaining to gynaecological laparoscopic day surgery. As can be observed in Tables $17 \& 18$ (p. 185), participants underwent slightly differing forms of this type of surgery. Therefore, for the vigilant coper undergoing a specific type of gynaecological laparoscopic surgery, even the extended information booklet with 'general' gynaecological laparoscopic information may have been insufficient.

The third and final aspect of hypothesis one concerns participants' coping styles i.e. distribution of avoidant coping and the spread of coping styles. Firstly, the distribution of avoidant coping behaviour was negatively skewed for both the original sample $(n=87)$ and the final sample $(n=51)$ (Figure 14 p.190 \& Figure 17 p.194). Both were deemed to be significantly negatively skewed and therefore not reflective of a normal distribution. This may be a tentative indication in the original sample $(n=87)$ of the level of apprehension experienced when undergoing day surgery and general anaesthesia i.e. such an anxiety-provoking event may for many people lead to extreme avoidant behaviour (high avoidance). However, avoidant coping behaviour in the final sample $(n=51)$ displayed a weak bi-modal distribution and a negative kurtosis 
i.e. flatter distribution curve. As the final sample only encompassed participants with vigilant and avoidant coping behaviours i.e. fluctuating and flexible copers were removed, the bi-modal appearance may be more indicative of the exclusive use of such extreme forms of coping. Indeed, it may be prudent in future studies only to enlist the help of participants with the more extreme forms of vigilant and avoidant coping behaviour in order to sharpen the focus of the potential outcomes i.e. only 40 participants employed from an original sample of 254 participants (Kohlmann et al 1996). The participants selected in the study by Kohlmann et al (1996) could have been highly vigilant and highly avoidant copers.

The second issue concerns the spread of coping behaviour. The participants' coping behaviours in the original sample $(n=87)$ were fairly evenly spread between vigilant, avoidant and fluctuating coping styles (Table 19 p.187). While it is recognised the data were collected from a specific group of gynaecological patients undergoing intermediate day surgery (and may not necessarily apply to all), this aspect of the data alone may be a strong indication of the need for differing levels of information provision. For example, if an information booklet provided by a day surgery unit contained only a simple explanation regarding proposed care and treatment then, based on the figures from this study, $54 \%$ of participants from the original sample would not have been satisfied with the booklet i.e. $28 \%$ of vigilant copers and $26 \%$ of fluctuating copers, totalling $54 \%$ (Table 19 p.187). This figure of 54\% could be a more accurate reflection of the dissatisfaction with day surgery information, highlighted earlier in the literature 
review (Chapter 4). Support for this also comes from a study by Kerrigan et al (1993) in which one quarter of all patients who were randomly given detailed information regarding possible complications following surgery stated they had received too much information. Although the percentages differ a little, a broad pattern could be emerging for day surgery patients i.e. 1 in 4 day surgery patients could possibly be vigilant copers and 1 in 3 could possibly be avoidant copers (Table 19 p.187). This has obvious implications for the production of future day surgery psycho-educational material. Future studies may therefore wish to investigate this aspect more closely in order to gain a more accurate picture i.e. are approximately $25 \%$ of all day surgery patients vigilant copers and approximately $33 \%$ avoidant copers? However, this must involve utilising a large sample of day surgery patients, as approximately $40 \%$ of the sample may be lost i.e. deemed fluctuating and flexible copers.

The extremes of this psycho-dynamic approach to coping i.e. vigilant and avoidant coping, are easier to assess, as they either require an extended level of information or a simple level of information, respectively. The percentage of participants with a fluctuating coping style was $26 \%$ or again approximately 1 in 4 ratio. Fluctuating copers falling into this more uncertain area inbetween the two extremes may be more difficult to satisfy on an informational basis, as their requirements are not as apparent. Rosenbaum and Piamenta (1998) in a study of patients scheduled for hernia repair referred to this group as experiencing 'dispositional conflict' i.e. a tendency to attend to and ignore threatening cues both at the same time. These participants were ".. rated by the nurses on the 
ward as the worse copers in comparison to all other groups of subjects..." (p. 841). Van Zuuren et al (1999) in a study of dental fears also suggested that such patients experience greater anxiety. Any anxiety management plan must therefore allow some flexibility within its boundaries for this less predictable although potentially sizeable group of patients. Future studies may therefore wish to investigate i) what additional information is commonly requested by a fluctuating coper i.e. problem focused and/ or emotional focused information, ii) at what stage in the proceedings extra information is commonly required i.e. few days before day of surgery, and iii) what other tangible methods of support may be required in order to assist patients in such a precarious state of anxiety arousal.

\section{Patient Anxiety}

Hypothesis two a) predicting when an individual with a vigilant coping style receives an extended level of pre-operative information, they will be less anxious than a vigilant coper in receipt of a simple level of pre-operative information, was rejected and the null hypothesis accepted. Hypothesis two b) predicting when an individual with an avoidant coping style receives a simple level of pre-operative information, they will be less anxious than an avoidant coper in receipt of an extended level of pre-operative information, was also rejected and the null hypothesis accepted.

However, three issues relating to hypothesis two arose and will be discussed further i.e. vigilant copers' self-rated anxiety prior to admission, the nurses' ratings of anxiety on the day of surgery and the interaction between coping 
style/ information booklet and the nurses' ratings of anxiety. The first aspect concerns an increase in self-rated anxiety for vigilant copers in comparison with avoidant copers i.e. a difference occurred between coping styles and not, as predicted, within coping styles. Approximately two days prior to admission for surgery there was a tendency for participants deemed as vigilant copers to rate themselves as experiencing greater state anxiety in comparison to avoidant copers, irrespective of the information booklet received. This marginally failed to reach the required level of statistical significance $(p=0.076)($ Table 29 p.203 \& Figure 18 p.204). Again, the utilisation of a larger sample may have produced an improved result. However, the nurses rated all participants in receipt of the simple information booklet, as more anxious $(p<0.05)$ (Table 30 p.205 \& Figure 19 p.206) (see below). The marginal finding above i.e. vigilant copers rating themselves as experiencing greater state anxiety $(p=0.076)$, together with all participants in receipt of the simple information being observed as more anxious, may cautiously indicate that it may have been the vigilant copers in receipt of the simple information who were the most anxious. The mean observed anxiety differed far more between vigilant copers in receipt of the simple and extended information booklets than the mean observed anxiety between avoidant copers in receipt of the two booklets (Table 30 p.205).

Such an increase in anxiety for vigilant copers has been suggested previously. In an extensive study by Miller et al (1988) the vigilant (monitoring) and avoidant (blunting) coping behaviour of patients with an acute medical condition was examined. It was concluded monitors were more likely than 
blunters to scan their bodies both internally and externally for threat relevant cues, perceive physical symptoms, interpret them negatively, become anxious and seek out medical advice. "Overall, high monitors demanded more tests, more counselling and more information from their doctor than did their low monitoring counterparts, but desired to play a less active role in their own care." (p. 146). Miller et al (1996) further suggested vigilant copers (monitors) with a medical condition ruminated continually i.e. experienced invasive negative thoughts. The vigilant copers in the present study may have experienced similar fears of day surgery because of their coping disposition and the absence of sufficient information from the hospital i.e. approximately $60 \%$ of all participants received no written information prior to admission. Additionally, the lack of detailed educational material within each information booklet may have caused greater anxiety for the vigilant coper (Health Locus of Control section).

The second aspect relates to observed anxiety and the information booklet received. Once admitted to hospital for surgery, participants who were only in receipt of the simple information booklet were rated by the nurses to be significantly more anxious than participants in receipt of the extended information booklet $(p<0.05)$ (Table 30 p.205 \& Figure 19 p.206). This occurred irrespective of coping style. However, the mean observed anxiety score differed more between vigilant copers in receipt of the differing information booklets. The mean observed anxiety score for avoidant copers only differed slightly between booklets. Therefore, vigilant copers may have been the more anxious when in receipt of the simple information booklet, although not 
significantly (Table 30 p.205). The nurses were completely blind to the type of information booklet each participant received and indeed their coping style.

Although not fully lending support for the stated hypothesis, such evidence presents a strong case for the provision of differing levels of information in modern surgery. Merely providing one level of simple information for day surgery patients may be a potential source of anxiety. Supplying all patients with an extended level of information could be suggested as the best option as no significant differences were established throughout the whole study between avoidant copers in receipt of the simple or extended information booklets (see below). However, providing one level of extended information to all patients may be an uneconomical use of resources i.e. financial cost of extended booklet production, medical and nursing staff time explaining potential unwanted information. Additionally, the extended booklets' propensity to increased anxiety will always remain i.e. when patients are obliged to listen to pre-operative information, anxiety may increase (Salmon 1992a, Kerrigan et al 1993).

The availability of two levels of information may therefore be strongly recommended to help prevent raised anxiety in day surgery patients although who should receive the extended level of information remains less clear. It may be appropriate to provide a choice of information i.e. a simple or extended information booklet, allowing each patient to choose and then discuss the information on an individual basis, adding supplementary details as and when required. Alternatively, a simple information booklet could be provided to everyone with additional information provision upon request. This would ensure 
the desired level of written and verbal information was attained. However, this would involve ensuring the process for gaining supplementary details was made explicit.

In addition to this second issue, 59\% of all avoidant copers received the extended information booklet. Therefore, some, if not all, of the avoidant copers in receipt of the extended information must have been less anxious as all participants in receipt of the extended information booklet were rated by the nurses as less anxious. Although it is uncertain how much of the extra information was read, it could be concluded the avoidant copers in receipt of the extended information may at some stage have read more than predicted and/ or desired more than predicted. Viewed broadly this was an unusual occurrence as it does not fully conform to the coping style theory i.e. avoidant copers only require minimal levels of information. However, it may be partially explained in the following three ways i.e. initial lack of information provided by the hospital, the possible selective reading of the extended information booklet and the unidentified criteria for the construction of information booklets containing differing levels of educational material.

Firstly, it could be an indication of avoidant copers requiring (and obviously in some cases not gaining) more information than had been received. Prior to admission only $40 \%$ of all participants in the study received some information from their consultant surgeon or day surgery unit. All participants, irrespective of their coping style, stated they wanted information prior to admission. Although not taken from a large sample population and thereby more open to questions 
of reliability, it may not be unreasonable to suggest the avoidant copers who received the extended information (59\% of avoidant copers), selected to read a little more for a better understanding of the surgery they were about to undergo, as too little had been received thus far. The exact time the additional information was read will always remain unknown. However, all avoidant copers in receipt of the extended information could have refrained from reading the supplementary details until a few days or even hours prior to admission.

Secondly, the avoidant copers who received the extended information booklet may have been highly selective, censoring the information yet still reading a little more than was available in the simple information booklet i.e. selective additional extracts. Once satisfied with the selected information little more may have been read. This may have hidden the avoidant copers' true level of anxiety. Avoidant copers in receipt of too much information in the extended information booklet may have only read what they wanted and were therefore not made more anxious. Indeed, it was observed by Salmon (1992a), in a study employing in-patient participants on a surgical ward when they were obliged to listen to pre-operative information, anxiety actually increased. The work of Salmon may therefore support this notion as the participants were a captive inpatient audience and some participants may have been avoidant copers i.e. obliged to listen and thereby made more anxious. Kerrigan et al (1993) also observed this when $25 \%$ of patients who were randomly given detailed information stated they had been given too much. However, the patients stated the additional, unwanted information had not increased their anxiety. Clearly, 
the patients in Kerrigan's study were not made more anxious because they did not fully read all the additional information, as they knew it would trigger an increase in anxiety. The avoidant copers may therefore have vetted the educational material they were reading.

For this reason, an increase in anxiety for a vigilant coper may be easier to measure than an increase in anxiety for an avoidant coper i.e. it may be more straightforward to detect the raised anxiety of a vigilant coper in receipt of too little information. It appears avoidant copers may simply self-select any information provided in order to evade potentially threatening material and then state (correctly for them), that their anxiety did not increase. Indeed, the mean observed anxiety for participants with an avoidant coping style differed little between possession of the extended information booklet and possession of the simple information booklet (Table 29 p.203).

The third issue relating to avoidant copers' apparent reduced anxiety when in receipt of the extended information relates to the construction of the information booklets. The researcher constructed the simple and extended information booklets. What constitutes 'simple' information provision and what constitutes 'extended' information provision is unclear. The simple information booklet sent out in this study could have been deemed 'too simple' and those who received it may have required more. Hence the need for some participants deemed avoidant copers, to read the extended booklet when it was sent to them. No guidelines were followed in the formulation of the information booklets utilised within the present study, as none exist. The researcher merely performed a 
detailed examination of a large number of hospital leaflets and constructed the booklets accordingly (Chapter 5). However, the simple information booklet utilised was superior in two main ways to the information currently provided to the vast number of day surgery patients i) the booklet contained a greater degree of problem focused and emotional focused coping information and, ii) it was provided prior to admission.

Future research studies may therefore wish to investigate the issues of avoidant coping behaviour and information provision in greater depth i.e. the level of information desired by avoidant copers, frequency of vetting educational material for anxiety provoking aspects by avoidant copers and the distinction between simple and extended information provision.

The final issue relating to hypothesis two concerns the interaction between participants' coping styles, information received and the nurses' ratings of anxiety. It was predicted the vigilant copers in receipt of the extended information booklet would be less anxious than the vigilant copers in receipt of the simple information booklet. It was also predicted the avoidant copers in receipt of the simple information booklet would be less anxious than the avoidant copers in receipt of the extended information booklet (Figure 6 p.144 \& Figure 7 p.145). This interaction, although failing to gain sufficient statistical significance $(p=0.15$ ), was indeed observed (Table 30 p.205 \& Figure 20 p.207). However, if avoidant copers do indeed vet all educational material as suggested above, a statistically significant interaction between avoidant coping, information booklet received and anxiety may never be observed. Only when 
forced to listen to extra information may such an interaction be viewed (Salmon 1992a, Kerrigan et al 1993). This observed interaction might also provide further tentative direction to the earlier issue in this section. Participants observed by the nurses to be the most anxious when in receipt of the simple information booklet, may have been the participants with a vigilant coping style (p. 236). Future studies may therefore wish to investigate this tentative claim in greater depth by utilising a far larger sample or a more targeted sample.

\section{Adjustment to Surgery}

Hypothesis three a) predicting when an individual with a vigilant coping style receives an extended level of pre-operative information, adjustment to surgery will be more positively influenced in comparison to a vigilant coper in receipt of a simple level of pre-operative information, was rejected and the null hypothesis accepted. Hypothesis three b) predicting when an individual with an avoidant coping style receives a simple level of pre-operative information, adjustment to surgery will be more positively influenced in comparison to an avoidant coper in receipt of an extended level of pre-operative information, was also rejected and the null hypothesis accepted.

No other issues relating to this hypothesis were uncovered. This may have been due, in part, to the brief measure employed in gauging adjustment to surgery and the inherent time constraints associated with day surgery. Firstly, adjustment to surgery was measured utilising an item within the Behavioural Adjustment Rating questionnaire completed by the nurses on the day of surgery (Appendix 11). Only one measure was employed due to the difficulty in gauging 
adjustment to surgery in the limited circumstances available. Previously within in-patient studies adjustment to surgery has been measured on numerous occasions during hospital admission i.e. questionnaires completed at differing times of hospital stay. This is obviously no longer practical when studying the day-case patient. Additionally, a series of measures have frequently been employed pre-operatively within in-patient studies in order to determine adjustment e.g. pre-operative coping behaviours, pre-medication required, length of anaesthesia and the amount of anaesthetic drugs required. Such indicators of adjustment to surgery are now clearly obsolete in modern day surgery as patients are admitted 1 to 2 hours prior to surgery, pre-medication is rare, general anaesthesia never exceeds 60 minutes and anaesthetic drug dosage is more accurate and almost exclusively determined by the weight of the patient. The number of emotional, behavioural and physiological measures available to the day surgery researcher will therefore increasingly be dictated by advances in medical practice and the inescapable time constraints inherent within day surgery.

Restricted time on the day of surgery may also have presented a challenge to the accuracy of data collection. The nurses were requested to observe their patients and complete the Behavioural Adjustment Rating questionnaire amid busy operating schedules. Consequently, they may have been unable to adequately assess their patient because of the pressure of dealing with essential physical intervention i.e. patients returning from theatre, dealing with post-operative morbidity and ensuring the next patient is ready for theatre. This 
brief, independent assessment may therefore have been a little too brief and a little too rushed for an adequate assessment of adjustment to surgery. In studies by Badner et al (1990) and Moerman et al (1996) it was revealed that anaesthetists were mediocre assessors of anxiety unless they had an increased amount of time in which to question the patient. This lack of time could likewise have hampered the nurses' assessment of adjustment to surgery. Indeed, for discussions regarding two well-documented periods of increased anxiety i.e. the operation and the anaesthetic, the nurses were unavailable. Seventy-two percent of nurses were absent when the patient spoke with the surgeon and $85 \%$ were absent when the patient spoke with the anaesthetist. The witnessing of such crucial interaction could have had the potential to strongly influence the nurses' view of their patients' adjustment to surgery. Future studies in this field of research may therefore wish to consider in great depth i) the indicators employed to measure adjustment to day surgery, ii) the time such measures may take for completion by the clinical staff and, iii) when such measures are to be completed by the clinical staff.

\section{Health Locus of Control}

Hypothesis four predicting when an individual with a vigilant or avoidant coping style receives the preferred level of pre-operative information, the desired level of health control will be more readily attained in comparison to a vigilant or avoidant coper in receipt of the non-preferred level of pre-operative information, was rejected and the null hypothesis accepted. 
As discussed in Chapter 5, locus of control has been considered as a 'fixed' aspect of personality by some researchers. However, a number of recent studies have established that in the healthcare situation, locus of control beliefs may be susceptible to change, especially the powerful others aspect (Johnston et al 1992, Avis 1994, Halfens 1995). Halfens (1995) concluded health locus of control beliefs were influenced by the environment when an individual was hospitalised, especially the powerful others aspect. Additionally, the influence of powerful others in uniform in such a situation could considerably enhance such beliefs. Milgram (1974) vividly demonstrated how powerful others in uniform could greatly influence perceptions and subsequently shape behaviour. Indeed, such evidence for a shift in healthcare locus of control behaviour may have been demonstrated within this study.

Two issues arose i.e. chance health locus of control/ participant coping style and external health locus of control (powerful others)/ participant coping style. The first aspect concerns an increase in chance health locus of control appraisals for vigilant copers. This narrowly failed to reach the required level of statistical significance $(p=0.11)$. Nevertheless, it does tentatively indicate that vigilant copers may have expected little choice or personal control over healthcare events, irrespective of information received (Table 33 p.212). Vigilant copers may have placed negative interpretations on numerous events within the healthcare setting because they perceived their ability to have some influence over decisions as a result of mere good fortune. The second issue may help to substantiate this point. 
The second issue concerns an increase in external health locus of control appraisal (powerful others), again for vigilant copers. Approximately two days prior to admission the external health locus of control appraisals of vigilant copers were significantly increased in comparison to avoidant copers, irrespective of the information booklet received $(p<0.05)$ (Table 34 p.214 \& Figure 22 p.216). Participants deemed vigilant copers therefore perceived little control over healthcare events judging powerful others to be determining proceedings. Such a shift in external health locus of control appraisal was only predicted for vigilant copers in receipt of the simple information booklet i.e. offer too little insight into events and limited participation in the decision-making process (Figure 11 p.149). Again, if a larger sample had been available this may have produced a statistically significant result i.e. vigilant copers may have experienced greater external health locus of control appraisals when only in receipt of the simple information booklet.

However, such an increase in external health locus of control appraisal by all vigilant copers may possibly be explained in two ways i.e. the lack of specific information within the information booklets and the enforced pre-operative hospital routines by powerful others in uniform. Firstly, the lack of specific information within both information booklets may have caused considerable concern for the vigilant coper. The information provided within the simple information booklet was very likely to have been insufficient. Likewise, the information provided in the extended booklet could have been deemed insufficient by the information hungry vigilant coper i.e. lack of specific detail. No 
guidelines were followed during the construction of the information booklets. The extended information booklet could therefore quite feasibly have been deemed as not containing enough information.

Both booklets were only concerned with general information regarding laparoscopic gynaecological surgery. No specific details regarding sterilisation, fertility investigations or investigations for pelvic pain were contained within both booklets i.e. the three main operations performed during laparoscopic gynaecological day surgery on participants within this study (Table 18 p.185). Any participant seeking more specific information regarding their precise laparoscopic gynaecological surgery may have been very disappointed with either booklet. A vigilant coper may therefore have viewed such considerable omissions as detrimental to their ability to make informed healthcare choices and establish a semblance of healthcare control. It is therefore possible that all participants deemed vigilant copers within this study did not receive sufficient information, either from the hospital (only $40 \%$ of all participants received written information) or from the information booklets sent by post in the pre-operative period. This being the case, a mismatch between vigilant coping style and information received would have occurred with both booklets. This would completely negate any attempt to explore the differences in the information requirements of vigilant copers. Indeed, as a match/ mismatch between coping style and information provision was central to all hypotheses, this may have contributed to a lack of significant outcomes concerning all vigilant coping behaviour. 
Secondly, the enforced pre-operative hospital routines may have provided a formidable impression of powerful others, determining healthcare events, with very little room for personal choice. The classic study by Milgram (1974) was earlier compared to the modern day surgery unit as a similar, powerful environmental situation may endure in the modern surgical arena. The vast majority of participants within this study attended a pre-assessment clinic prior to the day of surgery to check fitness for surgery. During this visit participants will have received numerous instructions as to what they must do in order to be allowed to undergo day surgery on the date and time available i.e. day surgery would only be authorised when the required steps were taken. Participants would have been instructed on many issues i.e. when to stop eating and drinking, when to arrive, what would happen to them on arrival, when they would be allowed to go home and what they must do once at home. The nurses, all in uniform, would have provided all such instructional details in an unfamiliar clinical environment. For convenience purposes, patients only visit the hospital once prior to the day of surgery. However, one visit does not allow adequate time for primary and secondary appraisal i.e. sufficient time in which to develop problem focused and emotional focused coping strategies. Additionally, the educational material provided by the hospital was limited as demonstrated by the $60 \%$ of participants who stated they had not received any written information prior to surgery. Therefore, mainly instructional information would have been provided by uniformed, powerful figures in an unfamiliar clinical 
setting, during one brief visit to the hospital with almost two-thirds of participants not being provided with written information.

Additionally, prior to a pre-assessment meeting with the nurse, all participants would have had a medical consultation with the senior surgeon undertaking the procedure. This medical consultation can occur many days/ weeks in advance of the pre-assessment check although increasingly it occurs immediately before the pre-assessment clinic visit so the patient does not have to visit the hospital on more than one occasion. During the medical consultation, participants would have been informed of the need for surgery and the early availability of a day surgery place. Frequently, if the surgical procedure is suitable for day surgery, patients are given little choice regarding the option of in-patient surgery. Again, the surgeon would have been wearing a uniform i.e. white coat, and directing the participants as to what will happen. At this stage all participants would have verbally consented to the surgery, again in the absence of much written information Therefore, when speaking to the medical staff much information regarding the stressful event of day surgery and general anaesthesia would also have been instructional i.e. participants notified of what will happen to them and what they must do to allow surgery to take place (Chapter $2 \& 3$ ).

The number of vigilant participants in this study who i) received no preoperative information from the hospital, ii) were in receipt of a simple information booklet or an extended information booklet with an insufficient amount of specific educational material and, iii) experiencing increased external health locus of control appraisals, must have been numerous. Therefore, many 
participants in this study must have experienced sub-optimal psychological care whilst undergoing day surgery. Without the information booklet provided within the bounds of this study, the level of information provided and overall psychoeducational care may have been even lower i.e. laparoscopic gynaecological day surgery patients do not normally receive such information booklets. Taking the number of vigilant copers encountered within this study as an approximate guide i.e. 1 to 4 ratio, in the region of $20-25 \%$ of all patients currently undergoing laparoscopic gynaecological day surgery in the United Kingdom may experience similar sub-optimal psychological care. Such pre-operative psychological circumstances cannot be considered conducive to a positive recovery from day surgery. Nor can such a situation provide the assurance of adequate psychological intervention for future hospital treatment i.e. external health locus of control appraisals may become even greater for vigilant copers as they can recall even more negative healthcare events. Indeed, such experiences may only seek to exacerbate matters, as ruminating over past events in such a pessimistic way, has been associated with a further increase in anxiety (Lok and Bishop 1999).

Future research studies may therefore wish to examine in greater detail i) the type and level of written information considered sufficient by a vigilant coper, ii) the degree to which the busy day surgery environment may negate attempts to establish individual aspects of control, and iii) the influence exerted over external health locus of control appraisals by uniformed day surgery doctors and nurses. 


\section{Self-efficacy}

Hypothesis five predicting when an individual with a vigilant or avoidant coping style receives the preferred level of pre-operative information, the perceived ability to cope will be more readily attained in comparison to a vigilant or avoidant coper in receipt of the non-preferred level of pre-operative information, was rejected and the null hypothesis accepted.

However, two issues relating to hypothesis five arose and will be discussed further i) the interaction between participants' coping styles, information received and self-efficacy, and ii) coping style and self-efficacy. Firstly, the interaction predicted in the hypothesis five, although failing to gain statistical significance was indeed observed i.e. vigilant copers in receipt of the extended information will experience greater self-efficacy and avoidant copers in receipt of the simple information will experience greater self-efficacy (Table 35 p.217 \& Figure 24 p.219). Again, the utilisation of a larger sample may have produced a significant result. However, for the avoidant coper, both the simple and extended information booklets may have provided the required level of information. This may have negated any attempt to explore the differences in the information requirements of avoidant copers. Additionally, the extended information booklet could have been judged by vigilant copers to be lacking in sufficient detail (Health Locus of Control section). If this were the case, a mismatch between vigilant coping style and information received could have occurred with both booklets. This would again completely negate any attempt to explore the differences in the information requirements of vigilant copers. 
Secondly, approximately two days prior to admission, the appraisal of selfefficacy for avoidant copers was significantly greater than for vigilant copers, irrespective of the information booklet received $(p<0.05)$ (Table 35 p.217). Participants deemed avoidant copers therefore believed they had a greater ability to cope with their day surgery experience. Such an increase in selfefficacy appraisal was predicted although only when an information provision/ coping style match occurred (Figure 12 p.150). For the reasons discussed in the 'Health Locus of Control' section above, avoidant copers may have gained the information they required from either booklet and considered themselves able to cope well with all events. Moreover, once in the surgical healthcare situation, it may have been deemed easy to elude information rather than to gain it, especially when the stay in hospital was to be so brief.

Conversely, appraisal of self-efficacy for the vigilant coper was low in comparison to the avoidant coper. Therefore, vigilant copers considered themselves less able to cope with their experience of day surgery. Additionally, once in the day surgery environment they may have deemed it too late or too difficult to gain supplementary information in order to enhance their ability to cope, especially when the stay was to be so brief. Therefore, certain individuals i.e. primarily vigilant copers, when faced with the prospect of day surgery may require much self-efficacy enhancement in order to compensate for their seemingly poor self-efficacy experience. Tangible aspects of psychological nursing intervention are therefore an extremely necessary part of day surgery 
care for such individuals, as the mere provision of general information, simple or extended, may have little impact.

When added to the issues detailed above regarding Health Locus of Control, the psychological experience of day surgery for numerous vigilant copers must have been quite basic i.e. sparse pre-operative information from the hospital, lack of specific educational material in either information booklet, increased external health locus of control appraisal and low self-efficacy. Indeed, low selfefficacy appraisal could have occurred because of the combined influence of reduced information and healthcare control. Additionally, although only tentatively suggested in the 'Patient Anxiety' section above, it would not be unreasonable to conclude that an individual with a vigilant coping style in such circumstances would also be quite anxious. Indeed, Litt et al (1999) in a dental fear study made similar claims. It was suggested dental surgery anxiety could be classified on three levels i.e. high anxiety, cue anxiety (anxiety derived by gaining site of dental equipment) and low anxiety. The main features of the high anxiety patients in comparison to the low anxiety patients was their reduced self-efficacy appraisals and frequent 'catastrophising' cognitions i.e. highly anxious patients always believed something was going to go seriously wrong (Litt et al 1999).

Future studies may therefore wish to explore the above aspects in more depth to determine why vigilant copers may experience lower self-efficacy appraisals in comparison to avoidant copers and how lower self-efficacy appraisals in such individuals may be enhanced. 


\section{Future Research}

Further research is required in order to help clarify a number of points. Firstly, a study which re-examines the proportion of vigilant and avoidant coping behaviour in day surgery patients is required. Approximately $25 \%$ of participants were deemed vigilant copers and $33 \%$ avoidant copers. It is important to verify such claims as this alone may provide evidence for considerable change regarding information provision within modern elective day surgery.

A similar study is also required, which employs in-patients as participants in order to assess the possible transfer of the recommendations, described in the next chapter, to elective in-patient surgery. If day surgery patients require aspects of psycho-educational care outlined, then in-patients may also require such care. Although in-patients obviously remain in hospital longer than day surgery patients, admission and discharge frequently occur within a few days.

A comparative study is required in which the psycho-educational management plan, outlined in Chapter 7 , is examined in conjunction with current day surgery arrangements. If such psycho-educational interventions are superior to the current arrangements then a comparative study may be able to highlight such differences. To help establish this comparison the newly validated Coping with Surgical Stress (Krohne et al 2000) questionnaire could be utilised. This questionnaire may also help to refine the suggested psycho-educational management plan. Additionally, a more in-depth interview of participants could be undertaken within a qualitative study to examine the pre and post-operative experiences of day surgery patients. This could possibly include men in the 
population sample as they were excluded from the present study. Including both men and women in the sample may be achieved by examining patients undergoing day surgery within differing surgical specialities i.e. orthopaedic surgery, genito-urinary surgery, dental surgery and general surgery.

If a similar study were to be undertaken, it is important to establish the criteria which determine the precise level of information required in simple and extended information booklets. This may be achieved in the preliminary stages by surveying vigilant and avoidant copers in order to detail their educational material requirements. In this way a precise picture of the required problemfocused and emotional-focused coping information can be gained. Also, during such a survey it may be of great benefit to determine the precise aspects of day surgery perceived by patients to be the most anxiety provoking. For example, it is known that general anaesthesia causes considerable anxiety. What is less well understood is the precise cause of anxiety i.e. needles, mask over face, loss of control.

Finally, vigilant copers, irrespective of the information received, experienced greater external health locus of control and lower self-efficacy appraisals. A study examining why such individuals may have inferior experiences of day surgery may help to uncover additional elements of psycho-educational nursing intervention. Such additional elements could help to establish the most effective care for such patients. 


\section{Study Limitations}

Two main aspects gave rise to limitations within the study. The first aspect concerns the study design and encompassed a number of issues i.e. sample size, surgical procedure performed, period in which the information booklet and questionnaire pack were sent, construction of one of the researcher designed questionnaires, anxiety questionnaire and information booklet construction. The second aspect concerns data collection and also encompassed a number of issues i.e. completion of the questionnaire by the nurses, brief amount of time available in which to collect data from participants undergoing day surgery, single researcher not being hospital based, the automatic exclusion of some participants and the contacting of potential participants by telephone.

The five issues regarding the limitations of the study design will commence this section. A larger sample size will be required for future research in this field. Approximately $33 \%$ of the sample were avoidant copers and $25 \%$ vigilant copers. Consequently, if a sample of avoidant copers is required, approximately $67 \%$ of all random participants may be lost immediately. If a random sample of vigilant copers is required, approximately $75 \%$ may be lost immediately. Other studies have encountered similar problems and comparable percentages. Within the present study, approximately $40 \%$ of the original sample was lost. Smaller sample sizes may render the final sample too small for inclusion within a factorial research design. Future studies may therefore wish to employ a larger sample in order to avoid the possibility of such problems. Alternatively, a larger sample population can be examined for coping behaviour and then only 
participants deemed vigilant and avoidant copers extracted and utilised within the study.

The second issue within the study design concerns the surgical procedure performed on participants. The study did not deal wholly and solely with one specific surgical procedure. Although all participants underwent laparoscopic gynaecological surgery, the actual surgical procedure differed somewhat i.e. sterilisation was the main procedural laparoscopic surgery and fertility treatment the main diagnostic laparoscopic surgery (Table 18 p.185). The utilisation of participants undergoing slightly differing surgical procedures may have permitted the introduction of extraneous variables. For example, undergoing differing types of laparoscopic gynaecological surgery may have led to differing levels of anxiety i.e. a discrete event such as laparoscopic sterilisation may have been deemed as less anxiety provoking than a laparoscopic investigation for fertility. The surgical procedure to be utilised within future research studies must, therefore, be both technically similar i.e. identical surgical procedures, and have a similar certainty of outcome i.e. high guarantee of a successful conclusion. Participants must not be left waiting for test results or be undergoing a series of surgical events, as may have occurred in the present study. The surgery must be a discrete event, permitting no variations.

The third issue within the study design concerns the time, prior to surgery, the information booklet and questionnaire pack were received. The information booklet and questionnaire pack may have been sent a little too soon in some instances. The booklet was sent 7 - 10 days prior to surgery to allow adequate 
time for information booklet reading and questionnaire completion. However, anxiety may have only become increased for some participants 2 - 3 days prior to surgery. The information booklet could have been read and the questionnaires completed 7 - 8 days prior to surgery. This may have allowed some participants to read the information and complete the anxiety questionnaire prior to an increase in anxiety.

The fourth issue within the study design concerns the design and evaluation of one of the researcher designed questionnaires. More time may have been required in the construction of the Participant Satisfaction Rating questionnaire in order to aid validation and reliability. It may have been beneficial to breakdown the questionnaire into its component parts and produce several questionnaires in order to prevent the problem of differing kinds of data i.e. items attempted to determine the amount of pre-operative information read, information gained while in the day surgery unit, information received from hospital personnel, most preferred time to receive information and the amount of post-discharge information read. The overall purpose of the questionnaire was to determine the amount of information pursued by participants. However, investigating multiple avenues of participant enquiry clearly led to the questionnaire becoming too diverse.

The fifth issue within the study design concerns the anxiety questionnaire by Marteau and Bekker (1992). This questionnaire was validated as a shortened form of the State-Trait Anxiety Inventory (STAI) (Spielberger et al 1983). However, the items within were very brief thereby providing little distinction 
between State and Trait anxiety. The questionnaire was chosen because it was brief and to be utilised alongside a number of other questionnaires. Additionally, the instructions to the questionnaire enquired as to how the participant felt 'now' i.e. asked about state anxiety only. As a result of these issues, the questionnaire was only utilised for State anxiety.

The sixth and final issue within the study design concerned the construction of the information booklets. The construction of the booklets may have inadvertently led to a number of limitations. A lack of general guidelines for the objective formulation of simple and extended information booklets may have caused discrepancies in the information levels provided. For example, the extended level of information may have been too brief and the simple level of information also too brief. As a match between participant coping style and the level of information received was a crucial part of the study, all attempts to make such a distinction may have been flawed.

The second main aspect concerns data collection and also encompassed a number of issues i.e. completion of the questionnaire by the nurses, brief amount of time available in which to collect data from participants undergoing day surgery, single researcher not being hospital based, the automatic exclusion of some participants and contacting potential participants by telephone.

The first issue concerning data collection pertains to the completion of the Behavioural Adjustment Rating questionnaire by the nurses during busy periods. The reduced amount of time available on the day of surgery possibly 
produced a conflict of priorities for the nursing staff involved. The nurses were obliged to make their brief observation of their patients and complete the short Behavioural Adjustment Rating questionnaire during very busy periods. This together with a 10 month data collection period, approximately three times each week may have produced a mild degree of dissatisfaction concerning continued data collection i.e. data collection fatigue. This may or may not have influenced questionnaire completion. Future studies may therefore wish to utilise more than one day surgery unit in order to avoid possible questionnaire completion fatigue during busy operating schedules.

The second issue concerning data collection relates to the brief amount of time available in which to collect data from participants undergoing minimal access surgery. This was, and will remain, a relentless limitation for the day surgery researcher. Within days of having undergone day surgery, participants were frequently fully mobile, back at work or pursuing their busy lives and thereby largely unavailable for interview. For example, the researcher telephoned one participant just 48 hours after her gynaecological laparoscopic surgery and general anaesthesia only to discover she had travelled to Blackpool Pleasure Beach, Lancashire, England to ride on the Pepsi Max (at the time of the study one of the worlds tallest Roller Coaster). A differing time for contact was therefore arranged with her relative. In comparison to previous in-patient studies in this area, the narrow time band in which to collect data is very restrictive. For the researcher in this field, this issue will increasingly become 
more challenging as the brief recovery period for intermediate day surgery falls even lower.

The third issue concerning data collection pertains to a single researcher not being hospital based. Only one researcher collecting the data prevented wider data collection. If more than one researcher had been involved, a larger sample could have been gained and more than one day surgery unit utilised. This may arguably have resulted in a more comprehensive picture and certainly would have helped the issue of sample size. Additionally, the researcher not being hospital based allowed numerous potential participants to be lost as not all bookings for day surgery came solely via the waiting list clerk i.e. further day surgery was arranged from the out-patient department directly with the day surgery unit.

The fourth and final issue concerning data collection relates to the automatic exclusion of some participants and contacting potential participants by telephone. A further restriction on the population sample was the automatic exclusion of all potential participants who did not have access to a telephone or did not leave a correct telephone number with their hospital records. This occurred on many occasions. Consequently a large number of participants were unable to be contacted. Additionally, despite attempting contact by telephone at differing times of the day, establishing initial contact with numerous participants was extremely difficult. The researcher was frequently aware of the relevant telephone numbers but no answers to the calls were gained. This resulted in the loss of many potential participants. 
In summary, the null hypothesis was accepted for all the stated hypotheses. However, the hypotheses examined variations within individual coping styles whereas a number of variations occurred between coping styles. The main themes concerned information provision, patient anxiety, adjustment to surgery, health locus of control and self-efficacy appraisal. Concerning information provision, the utilisation of a larger sample may have helped to alleviate some of the associated problems. Avoidant coping behaviour tended to be negatively skewed i.e. stronger avoidant behaviour. This may reflect the aversive nature of day surgery and general anaesthesia. Vigilant, avoidant and fluctuating coping styles were quite evenly spread. This aspect of the data only may be a strong indication of the need for differing levels of information.

When considering participant anxiety prior to admission there was a tendency for vigilant copers to rate themselves as more anxious than avoidant copers, irrespective of the information booklet received. However, this marginally failed to reach the required level of statistical significance $(p=0.076)$. Nonetheless, vigilant coping and increased anxiety became a reoccurring theme within the findings. Once admitted to hospital, participants in receipt of the simple information booklet were rated by the nurses to be significantly more anxious than participants in receipt of the extended information booklet $(p<0.05)$. The nurses observed this irrespective of coping style. Merely providing one level of simple information for day surgery patients may therefore be a potential source of anxiety. Two levels of information are recommended. Moreover, $59 \%$ of avoidant copers who received the extended information were also observed to 
be less anxious. This was an unusual occurrence, as it does not fully conform to the coping style theory. However, it may be explained by the possible lack of hospital information, the possible selective reading of the extended information booklet by avoidant copers and the unidentified criteria for the construction of information booklets with differing levels of educational material.

When considering participant adjustment to surgery no differences were established. This may have been due, in part, to the brief measure employed in gauging adjustment to surgery and the inherent time constraints. Measuring adjustment to day surgery may be limited as the methods in which to gauge adjustment are very restricted in modern surgery. Additionally, the pressure from vital physical nursing intervention associated with a busy operating schedule may have compromised the effective assessment of adjustment to surgery by the nursing staff.

When considering chance health locus of control appraisals for vigilant copers, the required level of statistical significance narrowly failed to be gained $(p=0.110)$. This finding occurred irrespective of information booklet received. This may cautiously indicate that vigilant copers could have experienced little choice and personal control over healthcare events. Strong support for this view arose from the vigilant copers' external health locus of control appraisals. The external health locus of control appraisal (powerful others) of vigilant copers was significantly higher $(p<0.05)$ in comparison to avoidant copers, irrespective of the information booklet received. Vigilant copers therefore believed they had little control over healthcare events viewing powerful others to 
be determining proceedings. Mainly instructional information was provided by uniformed, powerful figures in an unfamiliar clinical setting, during one brief visit to the hospital, prior to surgery where almost two-thirds of participants were not provided with written information. Such a healthcare environment may strongly influence an individual's perceived ability to exert some control over events. Additionally, the lack of detailed information within either information booklet may have limited the vigilant copers' ability to make any informed healthcare choices. The number of vigilant participants in this study who received no preoperative information from the hospital, had no specific information regarding their surgery and experienced increased external health locus of control appraisals, must have been numerous. Many participants must therefore have been exposed to sub-optimal psychological care whilst undergoing day surgery.

When considering the appraisal of self-efficacy, avoidant copers experienced a significantly higher level in comparison to vigilant copers, irrespective of the information booklet received $(p<0.05)$. Participants, deemed avoidant copers, therefore believed they had a greater ability to cope with their day surgery experience. Conversely, the appraisal of self-efficacy for the vigilant coper in comparison with the avoidant coper was lower. Collectively, the psychological experience of day surgery for vigilant copers must have been quite basic i.e. sparse pre-operative hospital information, lack of detailed educational material within either information booklet, increased external health locus of control appraisal, low self-efficacy and possibly increased anxiety. 
Future research into the psychological care of cay-case patients can be divided into three main areas i.e. participants, research design and rationale. Firstly, concerning participants, the number of vigilant and avoidant copers undergoing day surgery requires re-examination. Also, male patients require inclusion within a similar study, as do in-patients. Secondly, the recommendations, outlined in Chapter 7, require evaluation within a comparative study. Additionally, future studies may wish to pursue a more phenomenological approach. Finally, the anxiety provoking aspects of admission to hospital for surgery have been well documented. Less well known however are the specific causes of such anxiety.

The study limitations originated from two main areas i.e. study design and data collection. Firstly, it may have been prudent to deal wholly and solely with one surgical procedure and employ a larger sample. Also, the extended information booklet may have been too simple and questionnaire design too diverse. Additionally, the information booklet may have been sent out too soon i.e. prior to an increase in participant anxiety. The second aspect of study limitations concerned data collection. The amount of data collected by the nurses and the lack of time in which to collect it may have given rise to difficulties. Additionally, one researcher co-ordinating data collection, difficulty in contacting prospective participants and the automatic exclusion of some participants may have lead to the loss of many potential participants.

It is clear from the findings between coping styles and the information booklet received that differing levels of information provision, health locus of control and 
self-efficacy are of central importance to the effective psycho-educational care of the adult day surgery patient. An amalgamation of these findings has enabled the tentative construction of a modern pre-operative psycho-educational management plan. In the final chapter the recommended pre-operative psychoeducational management of an adult patient undergoing modern elective day surgery will be outlined utilising these central findings. 


\section{Chapter 7}

\section{Recommended Nursing Intervention}

Numerous changes are required to the psychological management of the adult elective day surgery patient as some may experience sub-optimal pre-operative psycho-educational care. Completely eliminating all anxiety, providing every aspect of information, constantly establishing the desired level of healthcare control and always enhancing self-efficacy for all adult day surgery patients is obviously an unrealistic goal. However, helping all patients to manage the psychological experience of, and recovery from, day surgery more effectively in the $21^{\text {st }}$ century is a very realistic and achievable goal. Proposals based on the significant findings between coping styles have therefore been put forward to advance the psycho-educational care received by the day surgery patient i.e. differing levels of information provision, health locus of control enhancement and self-efficacy enhancement. The way in which such psycho-educational care can be implementation has already been discussed in detail in Chapter 1 (Diagram 5 p.57a). However, some additional pragmatic steps to aid implementation of these proposals will be discussed.

\section{Psycho-educational Preparation for Day Surgery}

A more formal approach to pre-operative psycho-educational management is required to embrace the challenges discussed within the literature review and the significant findings established within this study. Furthermore, improved information requirements may soon become a necessity as a recent Audit Commission report (1998) stated "The overriding finding is that day surgery 
rates have increased very significantly for all 20 basket procedures." (p. 3 - 10) (Appendix 1). This is an indication of the continuing rise in the amount of day surgery being undertaken. The Audit Commission report (1998) also urged those Healthcare Trusts who currently undertake very little day surgery to increase their capacity. More recent, a further report by the Audit Commission (2001) surveyed over 300 day surgery units as part of an ongoing assessment. Six of the 10 measures or indicators employed to determine 'good practice' were concerned with the provision of information. The considerations necessary for the establishment of a more formal approach to pre-operative psychoeducational care in day surgery will be outlined below i.e. essential interventions and their clinical implementation.

Firstly, three aspects essential to the effective psycho-educational management of adult patients undergoing elective day surgery have arisen directly from the significant findings between coping styles. These themes are strongly recommended for implementation into day surgery nursing practice -
a) DifFerING LeVELS OF INFORMATION PROVISION
b) HeALTH Locus of CONTROL ENHANCEMENT
c) Self-efficacy enhancement

\section{A) Differing Levels of Information Provision}

Prior to admission vigilant copers rated themselves as more anxious than avoidant copers, irrespective of the information booklet received. However, this marginally failed to reach the required level of statistical significance $(p=$ 0.076). However, once admitted to hospital participants in receipt of the simple information booklet were rated by the nurses to be significantly more anxious 
than participants in receipt of the extended information booklet $(p<0.05)$. Although tentative, when added to the considerable evidence from the Literature Review this may indicate the strong desire of some patients (possibly vigilant copers) to receive an increased level of information. A number of studies have strongly suggested that forcing an extended level of information onto all patients can prove detrimental (Janis 1958, Salmon 1986; 1992a, Kerrigan et al 1993, Hawkshaw 1994, Lamarche et al 1998). A minimum of two levels of information is therefore highly recommended i.e. simple and extended information. Alternatively, all day surgery patients could be provided with a simple information booklet and supplementary details on request. The correct level of information provision is of crucial importance for the effective pre-operative psycho-educational management of adult patients prior to day surgery (Chapter 4). As the ability to gain the desired level of information prior to day surgery is extremely limited (as is gaining any information) a deliberate, planned effort on the part of the medical and nursing staff is required.

\section{B) Health Locus of Control Enhancement}

Chance health locus of control appraisals for vigilant copers narrowly failed to reach the required level of statistical significance $(p=0.11)$. However, this may cautiously indicate that vigilant copers could have expected to have little choice and little personal control over healthcare events. This view is supported by the external health locus of control appraisals for vigilant copers. Here there was a significant increase in external health locus of control appraisal for vigilant copers in comparison to avoidant copers, irrespective of the information booklet 
received $(p<0.05)$. Therefore, vigilant copers did not feel they had any control over events. They believed powerful others i.e. doctors and nurses, were more in control of healthcare proceedings. Providing the desired level of information throughout the whole surgical experience together with elements of personal inclusion in the decision-making process could possibly have a beneficial effect i.e. reduce external health locus of control appraisal, increase availability of problem focused and emotion focused coping strategies, provide realistic expectations. As the ability to exercise some control in an acute day surgery situation is limited, a deliberate, planned effort on the part of the medical and nursing staff is required. Additionally, vigilant copers may require an extended level of highly specific information as the general extended information booklet utilised within this study may have been insufficient for their educational requirements.

\section{C) Self-Efficacy Enhancement}

Avoidant copers experienced a significantly higher level of self-efficacy appraisal in comparison to vigilant copers, irrespective of the information booklet received $(p<0.05)$. Vigilant copers therefore expressed diminished coping abilities. Tangible psychological nursing intervention is therefore required to help such patients cope more effectively with the experience of day surgery (Table 36 p.273). Providing the desired level of information may be beneficial but clearly additional tangible aspects of psycho-educational nursing intervention are required i.e. positive self-efficacy enhancement. As the perceived ability to cope with day surgery is evidently reduced in some patients 
and much recovery occurs at home, a deliberate, planned effort on the part of the medical and nursing staff to enhance self-efficacy appraisal is required.

TABLE 36

PSycho-Educational management PLAN

\begin{tabular}{|c|l|}
\hline INTERVENTION & \multicolumn{1}{c|}{ RATIONALE } \\
\hline $\begin{array}{c}\text { Provision of } \\
\text { Differing Levels of } \\
\text { Information. }\end{array}$ & $\begin{array}{l}\text { Some patients, possibly vigilant copers, may require } \\
\text { more information then is generally available in the } \\
\text { modern day surgery environment. Too little information } \\
\text { for some patients may increase anxiety. Therefore, in } \\
\text { such a healthcare environment where the opportunity } \\
\text { to gain the desired level of information is often minimal, } \\
\text { direct action by the medical and nursing staff is } \\
\text { essential. }\end{array}$ \\
\hline $\begin{array}{c}\text { Health Locus } \\
\text { of Control }\end{array}$ & $\begin{array}{l}\text { Some patients, especially vigilant copers, do not feel in } \\
\text { control of events in the healthcare situation. Therefore, } \\
\text { in an acute modern surgical environment where the } \\
\text { opportunity for such personal inclusion is often } \\
\text { minimal, a planned programme of healthcare control } \\
\text { enhancement by the medical and nursing staff is } \\
\text { essential. }\end{array}$ \\
\hline $\begin{array}{l}\text { Self-efficacy } \\
\text { Enhancement. }\end{array}$ & $\begin{array}{l}\text { Some patients, especially vigilant copers, perceive } \\
\text { their ability to cope in a modern surgical environment } \\
\text { as limited. Therefore, in such a healthcare situation } \\
\text { where much recovery occurs at home, a planned } \\
\text { programme of self-efficacy enhancement by the } \\
\text { medical and nursing staff is essential. }\end{array}$ \\
\hline
\end{tabular}

\section{Implementation into Day Surgery}

Viewed in its totality, a proposed psycho-educational management plan such as this will help to systematically co-ordinate tangible nursing activities to aid anxiety management and ensure a convergent approach to coping. Psychoeducational nursing care can then be delivered in the way suggested earlier i.e. a balance achieved between the desired level support and the actual level of support i.e. information provision, healthcare control enhancement and selfefficacy enhancement (Diagram 5 p.57a). No such pre-operative psychoeducational management plan currently exists although such formalised 
interventions are manifestly required for the future of modern day surgery. Indeed, in a recent study by Krohne et al (2000) specific anxiety management plans were recommended.

Extended information booklets (full disclosure) could be distributed on request to patients who require such material (possibly vigilant copers) and simple information booklets (standard disclosure) to patients who require less information (possibly avoidant copers). However, $26 \%$ of participants in this study were fluctuating copers (requiring a mixed level of information) and $15 \%$ were flexible copers (satisfied with any level of information). Some additional aspects of information provision must therefore be made available for such patients i.e. fluctuating copers, as it cannot be assumed all day surgery patients will desire the extreme forms of information i.e. simple and extended. A fluctuating coper could be provided with the standard disclosure booklet plus additional requested elements from an 'intermediate' booklet.

The provision of the identified level of information is necessary throughout the whole day surgery experience i.e. pre and post-operatively. For the patient who initially requests a standard level of disclosure but subsequently requires more information in the post-operative period (and indeed for all patients in the postoperative period), a nurse initiated telephone call system 24 to 48 hours following surgery could be established. Many studies have vigourously supported such a move as pockets of undisclosed information can be quickly disseminated (Lewin and Razis 1995, De Jesus et al 1996, Wedderburn et al 
1996, Willis et al 1997, Heseltine and Edlington 1998, MacAndie and Bingham 1998).

Tangible aspects of psychological intervention must be devised and constructed within individual day surgery units i.e. aspects of intervention that help to enhance internal health locus of control appraisal and self-efficacy appraisal. Common post-operative issues could also be debated and solutions put forward. Complex interventions are not always required as simple, brief aspects of nursing intervention can enhance healthcare control and self-efficacy appraisal. For example, Mills and Krantz (1979) stated healthcare control need only be minor or 'real or perceived`as positive appraisals of control were gained when providing blood donors with a simple choice of which arm to use during the procedure. Additionally, Litt et al (1995) illustrated that mere verbal persuasion or the patient being falsely informed of their skilful ability to relax could enhance self-efficacy appraisal.

An increase in the pre-operative visiting period may assist primary and secondary appraisal of the situation. Such an option may also enhance choice and the sharing of information regarding coping at home following surgery. This pre-operative visit could consist, for those who require it, of a brief day surgery unit tour, video presentation, discussion with members of staff and provision of the desired information booklet. Moreover, the highly specific information concerning care and treatment possibly required by the vigilant coper could be provided during such visits. Visiting to discuss surgery in this way and later re- 
establishing contact with a familiar nurse on the day of surgery has helped to reduce anxiety (Vogelsang 1990).

Little in the way of extra resources would be required to implement the above plan as such a system could be established alongside existing practices. The formulation of the differing information booklets, a degree of staff training and a staged period of introduction would be the main requirements. Also, the cost of such a plan may not be prohibitive i.e. booklet production and resource costs, as it may be possible to utilise the British Association of Day Surgery's website (www.bads.co.uk) and/ or the International Association of Ambulatory Surgery's website (www.iaas-med.org). Differing levels of information could be established centrally and downloaded by individual day surgery units or indeed, the patients themselves whenever required. However, if such a system was to be launched nationally to include the British Association of Day Surgery (BADS) website then greater planning would be required i.e. formulation of nationally recognised information, BADS website preparation, broad agreement in terms of extended and simple information provision, consumer group evaluation, day surgery unit pilot sites and patient evaluation. Such innovative methods of communicating with patients are supported by a recent NHS Report entitled 'Shifting the balance of power within the NHS' (2001). This is one of numerous reports emerging from the Government's NHS Plan (2000), which recommend the wider use of technological advances to augment patient communication.

For legal and surgical consent purposes all patients must receive a certain level of information prior to surgery (Kaufmann 1983, Redman 1993, Whitwam 
1994, Kent 1996). The legal minimum requirements could become the starting point for both extended and simple information booklet construction as no criteria defining extended and simple information booklet construction currently exists. The Clinical Negligence Scheme for Trusts (CNST) has 11 standards, which must be adhered to in order for Healthcare Trusts to gain insurance against medical negligence claims. Only one standard $\left(\mathrm{N}^{0 .} 7\right)$ relates to information provision (Table 37 p.278). As can be seen, all information provided must contain between 10 and 20 risk/ benefits common to elective surgical treatment, depending upon the level of legal cover required. In addition, patients must be given instructions on how to obtain additional information, if desired. Between 10 and 20 general risks/ benefits could be provided covering a broad range of surgical procedures i.e. gynaecology, orthopaedics, dental surgery, urology. For the avoidant coper this could be very brief. Conversely, for the vigilant coper it could be made extensive and explicit. On an Internet website[s], this could be easily achieved i.e. pages for detailed information and pages for simple information. The use of such new technological methods are increasingly being incorporated within general health education to likewise avoid the customary 'one-type-fits-all' approach to information provision (Holt et al 2000). 
STANDARD 7 - ClinICAL NEGLIGENCE SCHEME FOR TRUSTS.

\section{INFORMATION ON THE RISKS AND BENEFITS OF PROPOSED TREATMENT OR} INVESTIGATION.

1) There is patient information available showing the risks/ benefits of 10 common elective treatments (minimal cover).

2) All consent forms used comply with NHSE Guidelines for design and use (maximal cover).

i) There is patient information available showing the risk/ benefits of 20 common elective treatments.

ii) There is a policy/ guideline stating that consent for elective procedures is to be obtained by a person capable of performing the procedure.

3) There is a clear mechanism for patients to obtain additional information about their condition.

Sanderson (1998)

A number of modifications will, however, be required to the psychoeducational management of day surgery patients in order to accommodate these innovative changes. Firstly, and most importantly, alongside the essential medical pre-assessment checks, patients must be able to decide which level of information they require. Time consuming questionnaires will not be required for this, merely an overview of the information available or the 'Information Pathways' available. A chart could be displayed on a wall within the preassessment clinic, highlighting these information pathways i.e. pathway one avoidant coper requiring a standard level of information, pathway two fluctuating coper requiring an intermediate level of information and pathway three - vigilant coper requiring an extended level of information (Table 38 p.279). 


\begin{tabular}{|c|c|c|c|}
\hline & \multicolumn{3}{|c|}{ I NFORMATION PATHWAYS } \\
\hline & $\begin{array}{c}\text { O N E } \\
\text { Avoidant Coper } \\
\text { (stANDARD DISCLOSURE) }\end{array}$ & $\begin{array}{l}\text { T W O } \\
\text { Fluctuating Coper } \\
\text { (INTERMEDIATE } \\
\text { DISCLOSURE) }\end{array}$ & $\begin{array}{c}\text { T H R E E } \\
\text { Vigilant Coper } \\
\text { (FULL DISCLosuRE) }\end{array}$ \\
\hline $\begin{array}{c}\text { Pre- } \\
\text { assessment } \\
\text { clinic visit }\end{array}$ & $\begin{array}{l}\text { Simple verbal/ written, } \\
\text { problem focused and } \\
\text { emotional focused } \\
\text { information concerning } \\
\text { treatment, care and } \\
\text { recovery. }\end{array}$ & $\begin{array}{l}\text { Intermediate, verbal/ } \\
\text { written, problem } \\
\text { focused and emotional } \\
\text { focused information } \\
\text { concerning treatment, } \\
\text { care and recovery. }\end{array}$ & $\begin{array}{l}\text { Extended verbal/ } \\
\text { written, problem focused } \\
\text { and emotional focused } \\
\text { information concerning } \\
\text { treatment, care and } \\
\text { recovery. }\end{array}$ \\
\hline $\begin{array}{l}\text { Day of } \\
\text { surgery }\end{array}$ & $\begin{array}{l}\text { Verbally reiterate - } \\
\text { simple problem focused } \\
\text { and emotional focused } \\
\text { information. }\end{array}$ & $\begin{array}{l}\text { Verbally reiterate - } \\
\text { intermediate problem } \\
\text { focused and emotional } \\
\text { focused information. }\end{array}$ & $\begin{array}{l}\text { Verbally reiterate - } \\
\text { extended problem } \\
\text { focused and emotional } \\
\text { focused information. }\end{array}$ \\
\hline $\begin{array}{l}\text { Following } \\
\text { discharge }\end{array}$ & $\begin{array}{l}\text { Verbal/ written simple } \\
\text { problem focused and } \\
\text { emotional focused } \\
\text { information for home } \\
\text { use. }\end{array}$ & $\begin{array}{l}\text { Verbal/ written } \\
\text { intermediate problem } \\
\text { focused and emotional } \\
\text { focused information for } \\
\text { home use. }\end{array}$ & $\begin{array}{l}\text { Verbal/ written extended } \\
\text { problem focused and } \\
\text { emotional focused } \\
\text { information for home } \\
\text { use. }\end{array}$ \\
\hline
\end{tabular}

This type of visual display will elicit a speedy reply as most patients are fully aware of their information requirements (coping style) and have previously been able to quickly identify the level of information appropriate for them (Mitchell 1997). Once chosen, the corresponding level of information could be provided in commercially produced booklets, video-taped programmes, database systems or via specific Internet websites e.g. British Association of Day Surgery, International Association of Ambulatory Surgery. In order to ensure the correct pathway is followed on the day of surgery a simple identification system could be established. For example, a simple traffic light colour coding system could be utilised i.e. 


$\begin{array}{lllll}\text { RED AVOIDANT COPER } & \rightarrow & \text { STOP } & \rightarrow & \begin{array}{c}\text { Provide standard } \\ \text { information only. }\end{array} \\ \text { AMBER FLUCTUATING COPER } & \rightarrow & \text { CAUTION } & \rightarrow & \begin{array}{c}\text { Provide intermediate } \\ \text { information. }\end{array} \\ \text { GREENVIGILANT COPER } & \rightarrow & \text { GO } & \rightarrow & \begin{array}{c}\text { Provide extended } \\ \text { information. }\end{array}\end{array}$

This system could take the form of an inexpensive, appropriately coloured wrist tag or marker for the back of the hand, bed or trolley i.e. RED $\rightarrow$ STOP $\rightarrow$ AVOIDANT COPER - provide little information; AMBER $\rightarrow$ CAUTION $\rightarrow$ FLUCTUATING COPER - provide intermediate information or information in additional specific area[s]; GREEN $\rightarrow$ GO $\rightarrow$ VIGILANT COPER - reveal all information in detail. The whole process would only take a matter of minutes to execute, its simplicity ensures little staff training is required, it would benefit patients enormously, provide a greater sense of satisfaction for the medical and nursing staff, save a considerable amount of time explaining unwanted or unnecessary information to highly anxious patients and crucially, be solely based on contemporary research evidence.

In order to achieve this method of working, information provision within the pre-operative period would need to be presented in a more systematic manner to ensure an information provision/ coping style match with the minimum amount of disruption i.e. choice of information level always decided prior to the day of surgery in the pre-assessment clinic and the relevant information made available. Once an information provision/ coping style match has been established the information booklet containing the correct level of problem- 
focused information (procedural, behavioural and sensory information) and emotional-focused information (cognitive coping strategies, relaxation and modelling) could be provided. All written material could then be discussed with the patient either in the pre-assessment clinic or by telephone prior to surgery. This level of information should continue through to discharge i.e. patient sent home with the appropriate level of information.

The implementation of such a system would possibly require a slow introduction to one surgical speciality or even one surgical procedure at a time. This may be necessary, as it would involve devising differing levels of information for each surgical procedure and a degree of staff training. Also, such changes may require a stable and sustained partnership between nurse clinicians and nurse educators as the introduction of innovative clinical research can be a very challenging endeavour for all concerned (Hunt 1987).

Finally, a recent report by the Audit Commission (1998) suggested certain people within the surgical arena i.e. nurses, should be identified as the coordinators of information provision in order to alleviate some of the numerous problems associated with information provision in day surgery (Chapter 4). The Royal College of Surgeons and Royal College of Psychiatrists (1997) also reiterated this notion of the nurse being the central figure in the provision of information to the day-case patient. However, in order for the nurse to become a central figure in day surgery information dissemination, the psychoeducational care required by the patient must firstly be determined i.e. a plan detailing the necessary nursing intervention. This was precisely the original aim 
of this study i.e. to discover the most suitable methods of psychologically preparing patients for modern day surgery and to formulate a pre-operative nursing plan which embraces such methods. If the proposals put forward in the N.H.S. Plan (2000) are to be realised i.e. $75 \%$ of all elective surgery undertaken on a day-case basis, such changes outlined here are essential.

\section{Summary of Recommendations}

The overall recommendations for day surgery nursing practice are based upon the significant findings established between coping styles and the information booklet. Three aspects of care are essential i) the provision and maintenance of the desired level of information, ii) ensuring a semblance of control and, iii) ensuring self-efficacy enhancement.

Problem-focused and emotional focused coping information (verbal and written) must be provided prior to the day of surgery either within the preassessment clinic, out-patient department or via the post. This information must be tailored to individual requirements. Some patients will require more information than others. The level required could be easily established by providing an element of choice in a prominent place i.e. choice of information pathways displayed in the pre-assessment clinic. Such a display of information could indicate three possible information routes i.e. standard, intermediate and extended disclosure for each surgical procedure -

$$
\begin{aligned}
& \text { STANDARD } \quad \text { simple information concerning treatment, care and } \\
& \text { recovery. } \\
& \text { INTERMEDIATE } \\
& \text { - simple/ detailed information concerning treatment, care } \\
& \text { and recovery. }
\end{aligned}
$$




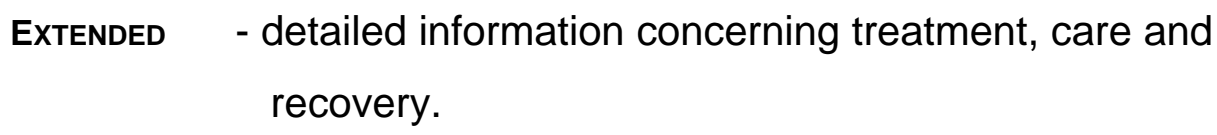

The information routes or pathways could be discussed and patients encouraged to choose the one most appropriate for them. Tangible aspects of psychological intervention are also required as merely providing the correct level of information is insufficient for effective psycho-educational management i.e. maintaining semblance of control and self-efficacy enhancement. Day surgery medical and nursing staff will be required to examine ways in which healthcare control can be enhanced. These need only be simple undertakings. If all staff were to frequently instigate simple measures, the desired level of healthcare control may be realised. Additionally, medical and nursing staff will be required to examine ways in which self-efficacy appraisal may be enhanced. Again, these techniques need only be simple undertakings on the part of all staff involved i.e. planned, sustained verbal encouragement.

On the day of surgery, ways in which the chosen information pathway i.e. standard, intermediate or extended information disclosure, can be maintained must be established. This is crucial as some patients, possibly vigilant copers, may experience greater anxiety when not fully aware of all events. An instant means of identifying the selected pathway of desired information will make such a task far easier e.g. appropriately coloured wrist tag, coloured skin marker on back of the hand, bedside identification note. In this way all staff involved in day surgery will be able to immediately identify the patients' educational 
requirements e.g. nurses, surgeons, anaesthetists, theatre staff, medical and nursing students.

On discharge the patient should be provided with the desired level of verbal and written information i.e. standard, intermediate and extended information disclosure. Again, this should be in the form of problem-focused and emotional focused coping information. The desired level of information is vital as a considerable amount of recovery occurs at home. Finally, as little time is spent in hospital and a great deal of time is spent recovering beyond the immediate reach of the day surgery unit, the promotion of healthcare control and enhancement of self-efficacy are essential upon discharge. 


\section{References}

Aasboe, V., Raeder, J. C. and Groegaard, B. (1998) Betamethasone reduces post-operative pain and nausea after ambulatory surgery. Anaesthesia Analgesia. Vol.87 No.2 p. $319-323$.

Agboola, O., Davies, J. and Davies, C. (1998) Laparoscopic sterilisation : The immediate and long term post-operative side effects using Bupivacaine infiltration and Diclofenac. Journal of One-Day Surgery. Vol.8 No.3 $\quad$ p. 7 $-9$.

Alexander, M. F., Fawcett, J. N. and Runciman, P. J. (1994) Nursing Practice Hospital and Home : The Adult Patient. London : Churchill Livingstone.

Alkaissi, A., Stålnert, M. and Kalman, S. (1999) Effect and placebo effect of acupressure (P6) on nausea and vomiting after outpatient gynaecological surgery. Acta Anaesthesiologica Scandinavica. Vol.43 No.3 p. 270 274.

Anderson, E. A. (1987) Pre-operative preparation for cardiac surgery facilitates recovery, reduces psychological distress and reduces the incidence of acute post operative hypertension. Journal of Consulting and Clinical Psychology. Vol.55a No.4 p. $513-520$.

Antonovsky, A. (1991) The structural sources of salutogenic strengths. In : Cooper, C. L. and Payne, R. (eds.) Personality and Stress : Individual Differences in the Stress Process. Chichester : Wiley and Sons. Ch. 4.

Antonovsky, A. (1992) Janforum : Locus of control theory. Journal of Advanced Nursing. Vol.17 No.8 p. $1014-1015$.

Audit Commission for Local Authorities and the National Health Service in England and Wales. (1990) A Short Cut To Better Services. Day Surgery in England and Wales. London : HMSO.

Audit Commission (1992) All in a day's work : An audit of day surgery in England and Wales. No.4 HMSO : London.

Audit Commission (1993) What seems to be the matter : Communication between hospitals and patients. HMSO : London.

Audit Commission for Local Authorities and the National Health Service in England and Wales (1998) Day surgery follow-up : progress against indicators from 'A Short Cut to Better Services'. London : HMSO.

Audit Commission for Local Authorities and the National Health Service in England and Wales (2001) Day surgery : review of national findings. No.4 London : HMSO. 
Auerbach, S. M. (1989) Stress management and coping research in the health care setting : An overview and methodological commentary. Journal of Consulting and Clinical Psychology. Vol.57 No.3 p. 388 - 395.

Augustin, P. and Hains, A. A. (1996) Effects of music on ambulatory surgery patients' pre-operative anxiety. American Operating Room Nurses' Journal. Vol.63 No.4 p. $750-758$.

Averill, J. R. and Rosenn, M. (1972) Vigilant and non-vigilant coping strategies and psycho-physiological stress reactions during the anticipation of electric shock. Journal of Personality and Social Psychology. Vol.23 No.1 p. 128 - 141.

Avis, M (1994) Choice cuts : an exploratory study of patients' views about participation in decision-making in a day surgery unit. International Journal of Nursing Studies. Vol.31 No.3 p. $289-298$.

Badner, N. H., Nielson, W. R., Munk, S., Kwiatkowska, C. and Gelb, A. W. (1990) Pre-operative anxiety : detection and contributing factors. Canadian Journal of Anaesthesia. Vol.37 No.4 p. $444-447$.

Baldacchino, D and Draper, P. (2001) Spiritual coping strategies : A review of the nursing research literature. Journal of Advanced Nursing. Vol.34 No.6 p. $833-841$.

Bandura, A. (1977) Self-efficacy : towards a unifying theory of behavioural change. Psychological Review. Vol.84 Vol.2 p. $191-215$.

Bandura, A. (1982) Self-efficacy mechanism in human agency. American Psychologist. Vol.37 No.2 p. $122-147$.

Bartlett, E. E. (1990) Putting patient education back into control. Patient Education and Counselling. Vol.16 No.3 p. $185-186$.

Baskerville, P. A., Heddle, R. M. and Jarrett, P. E. M. (1985) Preparation for surgery : information tapes for the patient. Practitioner. Vol.229 p. $677-678$.

Baume, R. M., Croog, S. H. and Nalbandian, J. (1995) Pain perception, coping strategies, and stress management among periodontal patients with repeated surgeries. Perceptual and Motor Skills. Vol.80 No. $1 \quad$ p. $307-319$.

Beddows, J. (1997) Alleviating pre-operative anxiety in patients : a study. Nursing Standard. Vol.11 No.37 p. 35 - 38.

Bhattacharya, S., Cameron, I. M., Mollison, J., Parkin, D. E. Abramovich, D. R. and Kitchener, H. C. (1998) Admission-discharge policies for hysteroscopic surgery : a randomised comparison of day case with in-patient admission. 
European Journal of Obstetrics \& Gynaecology and Reproductive Biology. Vol.76 No.1 p. $81-84$.

Birch, B. R. P., Chakraborty, R. and Miller, R. A. (1993) Anxiety in patients undergoing local anaesthetic day-case cystoscopy. Journal of One-Day Surgery. Vol.3 p. $15-17$.

Birch, B. R. P. (1994) Day case surgery and urology : present practice and $\begin{array}{llll}\text { future trends. British Journal of Urology. Vol.74 No.1 p. } 2-10 . & .\end{array}$

Birch, B. R. P. and Miller, R. A. (1994) Walk-in, walk-out day case genito-scrotal surgery with sedation reversal. A survey of patient attitudes and morbidity. British Journal of Urology. Vol.74 No. 5 p. $658-664$.

Bonanno, G. A., Davis, P. J., Singer, J. L. and Schwartz, G. E. (1991) The repressor personality and avoidant information processing : a dichotic listening study. Journal of Research in Personality. Vol.25 No.4 p. 386-401.

Bondy, L. R., Sims, N., Schroeder, D. R., Offord, K. P. and Narr, B. J. (1999) The effect of anaesthetic patient education on pre-operative patient anxiety. Regional Anesthesia and Pain Medicine. Vol.24 No.2 p. $158-164$.

Boore, J. R. P. (1978) Prescription for Recovery. London : Royal College of Nursing.

Bostrom, J., Crawford-Swent, C,. Lazar, N. and Helmer, D. (1994) Learning needs of hospitalised and recently discharged patients. Patient Education and Counselling. Vol.23 No.2 p. $83-89$.

Bostrom, J., Caldwell, J., McGuire, K. and Everson, D. (1996) Telephone followup after discharge from the hospital : does it make a difference? Applied Nursing Research. Vol.9 No.2 p. $47-52$.

Bottrill, P. (1994) Nursing assessment prior to day surgery. Journal of One$\begin{array}{llll}\text { Day Surgery. Vol.4 No.2 } & \text { p. } 22 \text { - } 23 .\end{array}$

Bradshaw, C., Pritchett, C., Bryce, C., Coleman, S. and Nattress, H. (1999) Information needs of general day surgery patients. Ambulatory Surgery. Vol.7 No.1 p. $39-44$.

Breemhaar, B. and Van den Borne, H. W. (1991) Effects of education and support for surgical patients : The role of perceived control. Patient Education and Counselling. Vol.18 No.3 p. $199-210$.

Brumfield, V. C., Kee, C. C. and Johnson, J. Y. (1996) Pre-operative patient teaching in ambulatory surgery settings. American Operating Room Nurses' Journal. Vol.64 No.6 p. $941-952$. 
Bubela, N., Galloway, S., McCay, E., McKibbon, A., Nagle, L., Pringle, D., Ross, E. and Shamian, J. (1990) The patient learning needs scale : reliability and validity. Journal of Advanced Nursing. Vol.15 No.10 p. $1181-1187$.

Buttery, Y., Sissons, J. and Williams, K. N. (1993) Patients' views one week after day surgery with general anaesthesia. Journal of One-Day Surgery. Vol.3 p. 6 - 8.

Byrne, D. (1961) The repression-sensitisation scale : rationale, reliability and validity. Journal of Personality. Vol.29 p. $334-349$.

Cahill, H. (1998) "It isn't what you do, but the way that you do it." : nurse $\begin{array}{llll}\text { practitioners in day surgery. Journal of One-Day Surgery. Vol.8 No.3 } & \text { J }\end{array}$ p. $11-14$.

Cahill, J. (1999) Basket cases and trollies : day surgery proposals for the millennium. Journal of One-Day Surgery. Vol.9 No.1 p. $11-12$.

Caldwell, L. M. (1991) The influence of preference for information on preoperative stress and coping in surgical out-patients. Applied Nursing Research. Vol.4 No.4 p. $177-183$.

Callesen, T., Bech, K., Nielsen, R., Anderson, J., Hesselfeldt, P., Roikjær, O. and Kehlet, H. (1998) Pain after groin repair. British Journal of Surgery. Vol.85 No.10 p. $1412-1414$.

Carver, C. S., Scheier, M. F. and Weintraub, J. K. (1989) Assessing coping : A theoretically based approach Journal of Personality and Social Psychology. Vol.56 No.2 p. $267-283$.

Carver, C. S. and Scheier, M. F. (1994) Optimism and health-related cognition : what variables actually matter? Psychology and Health. Vol.9 No.3 p. $191-195$.

Caunt, H. (1992) Pre-operative nursing intervention to relieve stress. British Journal of Nursing. Vol.1 No.4 p. $171-174$.

Christopherson, B. and Pfeiffer, C. (1980) Varying the timing of information to alter pre-operative anxiety and post-operative recovery in cardiac surgery patients. Heart and Lung. Vol.9 No.5 p. $854-861$.

Chung, F., Parikh, S., Theodorou, C., Dusek, B. and Cruise, C. (1994) Patient satisfaction with anaesthesia after day surgery. Anaesthesiology. Vol.181 No.3/ A p. A51.

Claxton, A. R., McGuire, G., Chung, F. and Cruise, C. (1997) Evaluation of morphine versus fentanyl for post-operative analgesia after ambulatory surgical procedures. Anaesthetic Analgesia. Vol.84 No.3 p. $509-514$. 
Clipperley, J. A., Butcher, L. A. and Hayes, J. E. (1995) Research utilisation : The development of a pre-operative teaching protocol. Medsurg Nursing. Vol.4 No.3 p. $199-206$.

Clyne, C. A. C. and Jamieson, C. W. (1978) The patient's opinion of day care vein surgery. British Journal of Surgery. Vol.65 No.3 p. $194-196$.

Codd, C. (1991) Are analgesics necessary for women at home following laparoscopic gynaecological day surgery? Nursing Practice. Vol.5 No.1 p. $8-12$.

Conner, M. and Norman, P. (1996) The role of social cognition in health behaviours. In : Conner, M. and Norman, P. (eds.) Predicting Health Behaviour. Buckingham : Open University Press. Ch. 1.

Cooper, J. M (1997) Day case cataract surgery in the UK and USA : a comparative study. British Journal of Nursing. Vol.6 No.1 p. $39-43$.

Cortis, J. D. and Lacey, A. E. (1996) Measuring the quality and quantity of information giving to in-patients. Journal of Advanced Nursing. Vol.24 No.4 p. $674-681$.

Coslow, B. I. F. and Eddy, M. E. (1998) Effects of pre-operative ambulatory gynaecological education: clinical outcomes and patient satisfaction. Journal of Peri-Anesthesia Nursing. Vol.13 No.1 p. 4-10.

Coulter, A. (1998) Evidence based patient information. British Medical Journal Vol.317 No.7153 p. 225 - 226.

Coulter, A., Entwistle, V. and Gilbert, D. (1998) Informing Patients. London : King's Fund.

Cozzarelli, C. (1993) Personality and self-efficacy as predictors of coping with abortion. Journal of Personality and Social Psychology. Vol.65 No.6 p. $1224-1236$.

Cruise, C. J. Chung, F., Yogendran, S. and Little, D. (1997) Music increases satisfaction in elderly outpatients undergoing cataract surgery. Canadian Journal of Anaesthesia. Vol.44 No.1 p. 43-48.

Crumlish, C. M. (1998) Coping strategies of cardiac surgery patients in the perioperative period. Dimensions of Critical Care Nursing. Vol.17 No.5 p. 272 $-278$.

Cundy, J. M. and Read, P. J. H. (1981) The acceptability of day stay for termination of pregnancy. British Journal of Clinical Practice. Vol.35 No.6 p. $215-218$. 
Cupples, S. A. (1991) Effects of timing and reinforcement of pre-operative education on knowledge and recovery of patients having coronary artery bypass graft surgery. Heart and Lung. Vol.20 No.6 p. $654-660$.

Daoud, Z. A. and Hasan, M. A. (1999) Day surgery : the effect of anxiety on induction of anaesthesia and insertion of the laryngeal mask airway. Journal of One-Day Surgery. Vol.9 No.2 p. $12-13$.

De Bruin, J. T., Schaefer, M. K., Krohne, H-W. and Dreyer, A. (2001) Preoperative anxiety, coping, and intra-operative adjustment : are there mediating effects of stress-induced analgesia? Psychology and Health. Vol.16 No.3 p. $253-271$.

De Groot, K. I., Boeke, S., Bonke, B. and Passchier, J. (1997a) A revaluation of the adaptiveness of avoidant and vigilant coping with surgery. Psychology and Health. Vol.12 No.5 p. 711-717.

De Groot, K. I., Boeke, S., van den Berge, H., Duivenvoorden, H. J. Bonke, B. and Passchier, J. (1997b) The influence of psychological variables on postoperative anxiety and physical complaints in patients undergoing lumbar surgery. Pain. Vol.69 No.1-2 p.19-25.

De Jesus, G., Abbotts, S., Collins, B. and Burvill, A. (1996) Same day surgery : results of a patient satisfaction survey. Journal of Quality in Clinical Practice. Vol.16 No.3 p. $165-173$.

De Lathouwer, C. and Poullier, J. P. (1998) Ambulatory surgery in 1994 - 1995 : The state of the art in 29 Organisation for Economic Co-operation and Development (OECD) countries. Ambulatory Surgery. Vol.6 No.1 p. 43 55.

Department of Health (1991) The Patient's Charter. London : HMSO.

Department of Health (2000) The N.H.S. Plan - Creating a $21^{\text {st }}$ Century N.H.S. London: HMSO (Cm 4818 - 1).

Department of Health (2001) Shifting the balance of power within the NHS. London : HMSO.

Devine, E. C. (1992) Effects of psycho-educational care for adult surgical patients : A meta-analysis of 191 studies. Patient Education and Counselling. Vol.19 No.2 p. $129-142$.

Diamond, B. (1995) When the nurse wields the scalpel. British Journal of Nursing. Vol.4 No.2 p. $65-66$. 
Dixon-Woods, M (2001) Writing wrongs? An analysis of published discourses about the use of patient information leaflets. Social Science and Medicine. Vol.52 No.9 p. $1417-1432$.

Doering, S., Kalzlberger, F., Rumpold, G., Roessler, S., Hofstoetter, B., Schatz, D. S., Behensky, H., Krismer, M., Luz, G., Innerhofer, P., Benzer, H., Saria, A. and Schuessler, G. (2000) Video-tape preparation of patients before hip replacement surgery reduces stress. Psychosomatic Medicine. Vol.62 No.3 p. $365-373$.

Domar, A. D., Noe, J. M. and Benson, H. (1987) The pre-operative use of the relaxation response with ambulatory surgery patients. Journal of Human Stress. Vol.13 No.3 p. 101- 107.

Done, M. L. and Lee, A. (1998) The use of a video to convey pre-anaesthetic information to patients undergoing ambulatory surgery. Anaesthetic Analgesia. Vol.87 No.3 p. $531-536$.

Donoghue, J., Pelletier, D., Duffield, C. and Gomez-Fort, R. (1995) Laparoscopic day surgery : the process of recovery for women. Ambulatory Surgery. Vol.3 No.4 p. $171-177$.

Donoghue, J., Pelletier, D., Duffield, C. and Torres, M. (1997) Australian men's experiences of cystoscopic day surgery. Ambulatory Surgery. Vol.5 No.1 p. $15-19$.

Donoghue, J., Pelletier, D., Duffield, C. and Torres, M. (1998) Australian men's experiences of cystoscopic day surgery (Part 2) Ambulatory Surgery. Vol.6 No.4 p. $189-196$.

Donovan, J. L. and Blake, D. R. (1992) Patient non-compliance : deviance or $\begin{array}{llll}\text { reasoned decision-making. Social Science and Medicine. Vol.34 No.5 } & \text { V }\end{array}$ p. $507-513$

Duits, A. A., Duivenvoorden, H. J., Boeke, S., Taams, M. A., Mochtar, B., Krauss, X. H., Passchier, J. and Erdman, R. A. M. (1999) A structural modelling analysis of anxiety and depression in patients undergoing coronary artery bypass graft surgery : A model generating approach. Journal of Psychosomatic Research. Vol.46 No.2 p. $187-200$.

Dunn, D. (1998) Pre-operative assessment criteria and patient teaching for ambulatory surgery patients. Journal of PeriAnesthesia Nursing. Vol.13 No.5 p. $274-291$.

Dusseldorp, E., Van Elderen, T., Maes, S., Meulman, J. and Kraaij, V. (1999) A meta-analysis of psycho-educational programmes for coronary heart disease patients. Health Psychology. Vol.18 No.5 p.506-519. 
Eachus, P. (1991) Multi-dimensional health locus of control in nurses. Journal of Advanced Nursing. Vol.16 No.2 p. $165-171$.

Edmondson, M. (1996) Patient Information. In Penn, S., Davenport, H. T., Carrington, S. and Edmondson, M. (eds.) Principles of Day Surgery. London : Blackwell Science. Ch. 5.

Edwards, N. D., Barclay, K., Catling, S. J., Martin, D. G. and Morgan, R. H. (1991) Day case laparoscopy : a survey of post-operative pain and an $\begin{array}{llll}\text { assessment of the value of diclofenac. Anaesthesia. Vol.46 No.12 } & \text { Nor }\end{array}$ p. $1077-1080$.

Egbert, L. D., Battit, G. E., Welch, C. E. and Bartlett, M. K. (1964) Reduction of post-operative pain by encouragement and instruction of patients. New England Journal of Medicine. Vol.270 No.16 p. $825-827$.

Elsass, P., Eikard, B., Junge, J., Lykke, J., Staun, P. and Feldt-Rasmussen, M. (1987a) Psychological effect of detailed pre-anaesthetic information. Acta Anaesthesiologica Scandinavica. Vol.31 No.7 p. $579-583$.

Elsass, P., Duedahl, H., Friis, B., Møller, I. W. and Bredgaard Sørensen, M. (1987b) The Psychological effect of having a contact-person from the anaesthetic staff. Acta Anaesthesiologica Scandinavica. Vol.31 No.7 p. 584 - 586.

Entwistle, V. A., Watt, I. S. and Sowden, A. J. (1997) Informative to facilitate patient involvement in decision-making - some issues. Journal of Clinical $\begin{array}{llll}\text { Effectiveness. } & \text { Vol.2 } & \text { No.3 } & \text { p. } 69-72 .\end{array}$

Fareed, A. (1994) A philosophical analysis of the concept of reassurance and its effects on coping. Journal of Advanced Nursing. Vol.20 No.5 p. $870-$ 873.

Fareed, A. (1996) The experience of reassurance : patients' perspectives. Journal of Advanced Nursing. Vol.23 No.2 p. $272-279$.

Fellowes, H., Abbott, D,. Barton, K., Burgess, L., Clare, A. and Lucas, B. (1999) Orthopaedic pre-admission assessment clinics. London : RCN.

Fenton-Lee, D., Cooke, T. and Riach, E. (1994) Patient acceptance of day surgery. Annals of the Royal College of Surgeons of England. Vol.76 No.5 p. $331-334$.

$\begin{array}{llll}\text { Firth, F. (1991) Pain after day surgery. Nursing Times. } & \text { Vol.87 No.40 }\end{array}$ p. $72-76$. 
Fitzpatrick, R. and Hopkins, A. (1983) Problems in the conceptual framework of patient satisfaction research : an empirical exploration. Sociology of Health and Illness. Vol.5 No.3 $\quad$ p. $297-311$.

Fitzpatrick, J. M., Selby, T. T. and While, A. E. (1998) Patients' experiences of $\begin{array}{lll}\text { varicose vein and arthroscopy day surgery. British Journal of Nursing. Vol.7 } & \text { Vor }\end{array}$ No.18 p. $1107-1115$.

Fleming, V. E. M. (1992) Client education : a futuristic outlook. Journal of Advanced Nursing. Vol.17 No.2 p. $158-163$.

Flood, A., Lorence, D. P., Ding, J., McPherson, K. and Black, N. A. (1993) The role of expectations in patient's reports of post-operative outcomes and improvement following therapy. Medical Care. Vol.31 Part 3 No.11 p. 1043 - 1056.

Folkman, S. (1984) Personal control and stress and coping process : A theoretical analysis. Journal of Personality and Social Psychology. Vol.46 No.4 p. $839-852$.

Folkman, S. and Lazarus, R. S. (1980) An analysis of coping in a middle-aged community sample. Journal of Health and Social Behaviour. Vol.21 No.3 p. $219-239$.

Folkman, S. and Lazarus, R. S. (1991) Coping and Emotions. In : Monat, A and Lazarus, R. S. (eds.) 3rd Ed. Stress and Coping : An Anthology. New York: Columbia University Press. p. 207 - 227.

Folkman, S., Lazarus, R. S., Dunkel-Schetter, C., DeLongis, A. and Gruen, R. J. (1986) Dynamics of a stressful encounter : cognitive appraisal, coping and encounter outcomes. Journal of Personality and Social Psychology. Vol.50 No.5 Pt.2 p. $992-1003$.

Foulger, V. (1997) Patients' views of day-case cardiac catheterisation. $\begin{array}{llll}\text { Professional Nurse } & \text { Vol.12 No.7 } & \text { p. } 478-480 .\end{array}$

French, H-P. (1979) Reassurance : A nursing skill? Journal of Advanced Nursing. Vol.4 No.6 p. $627-634$.

Friedlander, M. L., Steinhart, M. J., Daly, S. S. and Snyder, J. (1982) Demographic, cognitive and experimental predictors of pre-surgical anxiety. Journal of Psychosomatic Research. Vol.26 No.6 p. $623-627$.

Fraser, R. A., Hotz, S. B., Hurtig, J. B., Hodges, S. N. and Moher, D. (1989) The prevalence and impact of pain after day-care tubal ligation surgery. Pain. Vol.39 No.2 p. $189-201$. 
Frisch, S. R., Groom, L. E., Seguin, E., Edgar, L. J. and Pepler, C. J. (1990) Ambulatory surgery : a study of patients' and helpers' experiences. American Operating Room Nurses' Journal. Vol.52 No.5 p. $1000-1009$.

Fung, D. and Cohen, M. (2001) What do out-patients value most in their anesthesia care? Canadian Journal of Anesthesia. Vol.48 No.1 p. 12 - 19.

Gaberson, K. B. (1995) The effects of humorous and musical distraction on preoperative anxiety. Association of Operating Room Nurses' Journal. Vol.62 No.5 p. $784-791$.

Gagliano, M. E. (1988) A literature review on the efficacy of video in patient education. Journal of Medical Education. Vol.63 October p.785-792.

Gammon, J. and Mulholland, C. W. (1996) Effects of preparatory information prior to elective total hip replacement on psychological coping outcomes. Journal of Advanced Nursing. Vol.24 No.2 p. $303-308$.

Gamotis, P. B., Dearmon, V. C., Doolittle, N. O. and Price, S. C. (1988) Inpatient vs. outpatients satisfaction. American Operating Room Nurses' Journal. Vol.47 No.6 p. $1421-1425$.

Gattuso, S. M., Litt, M. D. and Fitzgerald, T. E. (1992) Coping with gastrointestinal endoscopy : self-efficacy enhancement and coping style. Journal of Consulting and Clinical Psychology. Vol.60 No.1 p. $133-139$.

Garcia-Ureňa, M. A., Garcia, M. V., Ruiz, V. V., Carnero, F. J., Huerta, D. P. and Jimenez, M. S. C. (2000) Anesthesia and surgical repair of aponeurotic hernias in ambulatory surgery. Ambulatory Surgery. Vol.8 No.4 p. 175 $-178$.

Garden, A. L., Merry, A. F., Holland, R. L. and Petrie, K. J. (1996) Anaesthesia information - what patients want to know. Anaesthesia Intensive Care. Vol.24 No.5 p. $594-598$.

Garraway, W. M., Cuthbertson, C. Fenwick, N., Ruckley. C. V. and Prescott, R. J. (1978) Consumer acceptability of day care after operations for hernia or varicose veins. Journal of Epidemiology and Community Health. Vol.32 No.3 p. $219-221$.

Gattuso, S. M., Litt, M. D. and Fitzgerald, T. E. (1992) Coping with gastrointestinal endoscopy : self-efficacy enhancement and coping style. Journal of Consulting and Clinical Psychology. Vol.60 No.1 p. $133-139$.

Ghosh, S. and Sallam, S. (1994) Patient satisfaction and post-operative demands on hospital and community services after day surgery. British Journal of Surgery. Vol.81 No.11 p. $1635-1638$. 
Gnanalingham, K. K. and Budhoo, M. (1998) Day case hernia repair under local versus general anaesthetic : patient preferences. Ambulatory Surgery. Vol.6 No.4 p. 227 - 229.

Goldmann, L., Ogg, T. W. and Levey, A. B. (1988) Hypnosis and day case anaesthesia. Anaesthesia. Vol.43 No.6 p. $466-469$.

Goodman, H. (1997) Patients' perceptions of their education needs in the first six weeks following discharge after cardiac surgery. Journal of Advanced Nursing. Vol.25 No.6 p. $1241-1251$.

Gould, D. and Wilson-Barnett, J. (1995) A comparison of recovery following hysterectomy and major cardiac surgery. Journal of Advanced Nursing. Vol.10 No.4 p.315-323.

Greenwood, M. (1993) Patients' views of oral day surgery. British Dental Journal. Vol.175 No.4 p. $130-132$.

Guadagnoli, E. and Ward, P. (1998) Patient satisfaction in decision-making. Social Science and Medicine. Vol.47 No.3 p. $329-339$.

Guilbert, E. and Roter, D. (1997) Assessment of satisfaction with induced abortion procedure. Journal of Psychology. Vol.131 No.2 p. 157-166.

Gupta, A., Larsen, L., Sjöberg, I. and Lennmarken, C. (1994) Out-patients survey : a survey of anaesthesia care in a university hospital. Scandinavian Journal of Caring Sciences. Vol.8 No.2 p. 107-112.

Haddock, J., Challands, A., Stevens, J., Wong, C. and Walters, S. (1999) Patient controlled oral analgesia at home (PCOAH) for the management of postoperative pain following day surgery. Journal of One-Day Surgery. Vol.8 No.4 p. $3-8$.

Haldane, G., Stott, S. and McMenemin, I. (1998) Pouch of Douglas block for laparoscopic sterilisation. Anaesthesia. Vol.53 No.6 p. 589-603.

Halfens, R. J. G. (1995) Effect of hospital stay on health locus of control beliefs. Western Journal of Nursing Research. Vol.17 No.2 p. 156- 167.

Hansen, C. H., Hansen, R, D. and Shantz, D. W. (1992) Repression at encoding : discrete appraisals of emotional stimuli. Journal of Personality and Social Psychology. Vol.63 No.6 p. $1026-1035$.

Harju, E. (1991) Patient satisfaction among day surgery patients in a central hospital. Quality Assurance in Health Care. Vol.3 No.2 p. 85-88. 
Hartfield, M. T., Cason, C. L. and Cason, G. J. (1982) Effects of information about a threatening procedure on patients' expectations and emotional distress. Nursing Research. Vol.31 No.4 p. 202 - 206.

Hartsfield, J. and Clopton, J. R. (1985) Reducing pre-surgical anxiety : A possible visitors effect. Social Science and Medicine. Vol.20 No.5 p. $529-533$.

Hathaway, D. (1986) Effect of pre-operative instruction on post-operative outcomes: A meta-analysis. Nursing Research. Vol.35 No.5 p. $269-275$.

Hawkshaw, D. (1994) A day surgery patient telephone follow-up survey. British Journal of Nursing. Vol.3 No.7 p. $348-350$.

Hayward, J. (1975) Information - A prescription against pain. Series 2 No.5 London : Royal College of Nursing.

Health Service Commissioner for England, for Scotland and for Wales. (1997) Annual Report for 1996 - 97. London : HMSO.

Health Service Commissioner for England, for Scotland and for Wales. (2001) Annual Report for 2000 - 2001. London : HMSO.

Heier, T. and Steen, P.A. (1996) Awareness in anaesthesia : incidence, consequences and prevention. Acta Anaesthesiologica Scandinavica. Vol.42 No.2 p. $484-485$.

Heikkilä, J., Paunonen, M., Laippala, P. and Virtanen, V. (1998) Nurses' ability to perceive patients' fears related to coronary arteriography. Journal of Advanced Nursing. Vol.28 No.6 p. $1225-1235$.

Heinen, C. and Melodie, P. (1992) "Operation Information" for ambulatory surgical patients. Nursing Management (U.S.A). Vol.23 No.8 64Q-64T.

Heseltine, K. and Edlington, F. (1998) A day surgery post-operative telephone call line. Nursing Standard. Vol.13 No.9 p. $39-43$.

Hill, B. J. (1982) Sensory information, behavioural instructions and coping with sensory alteration surgery. Nursing Research. Vol.31 No.1 p. $17-21$.

Hock, M., Krohne, H. W. and Kaiser, J. (1996) Coping dispositions and the processing of ambiguous stimuli. Journal of Personality and Social Psychology. Vol.70 No.5 p. $1052-1066$.

Hodge, D. (1994) Introduction to day surgery. Surgical Nurse. Vol.7 No.2 p. $12-16$. 
Hodge, D. (1999) Day Surgery : A Nursing Approach. London : Churchill Livingstone.

Holahan, C. J. and Moos, R. H. (1985) Life stress and health : Personality, coping, and family support in stress resistance. Journal of Personality and Social Psychology. Vol.49 No.3 p.739-747.

Holden-Lund, C. (1988) Effects of relaxation with guided imagery on surgical stress and wound healing. Research in Nursing and Health. Vol.11 No.4 p. $235-244$.

Holt, C. L., Clark, E. M., Kreuter, M. W. and Scharff, D. P. (2000) Does locus of control moderate the effects of tailored health education materials. Health Education Research. Vol.15 No.4 p. $393-403$.

Horne, D. J., Vatmanidis, P. and Careri, A. (1994) Preparing patients for invasive medical and surgical procedures 1 : adding behavioural and cognitive interventions. Behavioural Medicine. Vol.20 No.1 p. $5-13$.

Huang, A., Stinchcombe, C., Davies, M., Phillips, D. and McWhinne, D. L. (2000) Prospective five-year audit for day-case laparoscopic cholecystectomy. Journal of One-Day Surgery. Vol.9 No.4 p. $15-17$.

Hulme, J., Waterman, H. and Hillier, V. F. (1999) The effects of foot massage on patient's perception of care following laparoscopic sterilisation as day case patients. Journal of Advanced Nursing. Vol.30 No.2 p. $460-468$.

Hunt, M. (1987) The process of translating research findings into nursing practice. Journal of Advanced Nursing. Vol.12 No.1 p.101-110.

Hunt, L., Luck, A. J., Rudkin, G. and Hewett, P. J. (1999) Day-case haemorrhoidectomy. British Journal of Surgery. Vol.86 No.2 p. 255-258.

Icenhour, M. L. (1988) Quality interpersonal care : a study of ambulatory surgery patients' perspectives. American Operating Room Nurses' Journal. Vol.47 No.6 p. 1414 - 1419.

Ismail, W. (1997) Ambulatory hernia service : preliminary experience in a district general hospital. Journal of One-Day Surgery. Vol.7 No.2 p. 10-14.

Jamison, R. N., Parris, W. C. V. and Maxon, W. S. (1987) Psychological factors influencing recovery from outpatient surgery. Behaviour Research and Therapy. Vol.25 No.1 p. $31-37$.

Janis, I. L. (1958) Psychological Stress. Psychoanalytic and Behavioural Studies of Surgical Patients. New York : Wiley.

Jarrett, P. E. M. (1995) Day case surgery. Surgery. Vol.13 No.1 p. 5 - 7. 
Jarrett, P. E. M. (1997) Day case surgery : past and future growth. Surgery. Vol.15 No.4 p. $94-96$.

Jennings, B. M. and Sherman, R. A. (1987) Anxiety, locus of control, and satisfaction in patients undergoing ambulatory surgery. Military Medicine. Vol.152 No.4 p. $206-208$.

Jerusalem, M. and Schwarzer, R. (1992) Self-efficacy as a resource factor in stress appraisal process. In : R. Schwarzer (ed.) Self-Efficacy : Thought Control of Action. Washington, DC : Hemisphere.

Johnston, M. (1980) Anxiety in surgical patients. Psychological Medicine. Vol.10 No.1 p. $145-152$.

Johnston, M., Gilbert, P., Partridge, C. and Collins, J. (1992) Changing perceived control in patients with physical disabilities : An intervention study with patients receiving rehabilitation. British Journal of Clinical Psychology. Vol.31 No.1 p. 89 - 94.

Johnston, M. and Vogele, C. (1993) Benefits of psychological preparation for surgery: A meta analysis. Annals of Behavioural Medicine. Vol.15 No.4 p. $245-256$.

Kain, Z. N., Kosarussavadi, B., Hernandez-Conte, A., Hofstadter, M. B. and Mayes, L. C. (1997) Desire for peri-operative information in adult patients : a cross sectional study. Journal of Clinical Anesthesia. $\begin{array}{llll}\text { Vol.9 No.6 } & \text { p. } 467\end{array}$ -472 .

Kangas-Saarela, T., Ohukainen, J. and Koivuranta, M. (1999) Patients' experiences of day surgery - an approach to quality control. Ambulatory $\begin{array}{llll}\text { Surgery. Vol.7 No.1 } & \text { p. } 31-34 .\end{array}$

Kaufmann C. L. (1983) Informed consent and patient decision making : two decades of research. Social Science and Medicine. Vol.17 No.21 p. 1657 $-1664$.

Kelly, M. C. (1994) Patients' perception of day case surgery. Ulster Medical $\begin{array}{llll}\text { Journal. Vol.63 No.1 } & \text { p. } 27-31 .\end{array}$

Kempe, A. R. (1987) Ambulatory surgery. Association of Operating Room Nurses`Journal. Vol.45 No.2 p. $500-507$.

Kempe, A. R. and Gelazis, R. (1985) Patient anxiety levels. Association of Operating Room Nurses' Journal. Vol.41 No.2 p. $390-396$.

Kennedy, J. A. (1995) An audit of patients` problems after discharge from a day surgery unit. British Journal of Medical Economics. Vol.9 p. 51-53. 
Kent, G. (1996) Shared understandings for informed consent : The relevance of psychological research on the provision of information. Social Science and Medicine. Vol.43 No.10 p. $1517-1523$.

Kerrigan, D. D., Thevasagayam, R. S., Woods, T. O., McWelch, I., Thomas, W. E. G., Shorthouse, A. J. and Dennison, A. R. (1993) Who's afraid of informed consent? British Medical Journal. Vol.306 No.6873 p. $298-300$.

Kiecolt-Glaser, J. K., Marucha, P. T., Malarkey, W. B., Mercado, A. M. and Glaser, R. (1995) Slowing of wound healing by psychological stress. Lancet. Vol.346 No.8984 p. $1194-1196$.

Kiecolt-Glaser, J. K., Page, G. G., Marucha, P. T., MacCallum, R. C. and Glaser, R. (1998) Psychological influences on surgical recovery. American Psychologist. Vol.53 No.11 p. $1209-1218$.

King, B. (1989) Patient satisfaction surgery : day surgery unit. Australian Clinical Review. Vol.9 No.3-4 p. 127-129.

King, I. and Tarsitano, B. (1982) The effect of structured and unstructured preoperative teaching: A replication. Nursing Research. Vol.31 No.6 p. 324 $-329$.

King, K. B., Rowe, M. A., Kimble, L. P. and Zerwic, J. J. (1998) Optimism, coping, and long term recovery from coronary artery surgery in women. Research in Nursing and Health. Vol.21 No.1 p. $15-26$.

Klafta, J. M. and Roizen, M. F. (1996) Current understanding of patients' attitudes toward and preparation for anaesthesia : a review. Anesthesia Analgesia. Vol.83 No.6 p.1314-1321.

Kleinbeck, S. V. M., and Hoffart, N. (1994) Outpatient recovery after laparoscopic cholecystectomy. Association of Operating Room Nurses' Journal. Vol.60 No.3 p. $394-402$.

Kleinbeck, S. V. M. (2000) Dimensions of peri-operative nursing for a national specialty nomenclature. Journal of Advanced Nursing. Vol.31 No.3 p. 529 $-535$.

Knudsen, V. J. (1996) An audit of day surgery carers. Journal of One-Day Surgery. Vol.6 No.2 p. $5-6$.

Kohlmann, C-W, Weidner, G. and Messina, C. R. (1996) Avoidant coping style and verbal-cardiovascular response dissociation. Psychology and Health. Vol.11 No.3 p. 371 - 384. 
Kratz, A. (1993) Pre-operative education : preparing patients for a positive experience. Journal of Post Anesthesia Nursing. Vol.8 No.4 p. $270-275$.

Krause, N. and Stryker, S. (1984) Stress and well-being : The buffering role of locus of control beliefs. Social Science and Medicine. Vol.18 No.9 p.783 $-790$.

Krohne, H. W. (1978) Individual differences in coping with stress and anxiety. In : Spielberger, C. D. and Sarason, I. G. (eds.) Stress and Anxiety. Volume 5. London: Wiley. p. 233.

Krohne, H.W. (1989) The concept of coping modes : relating cognitive person variables to actual coping behaviour. Advances in Behaviour Research and Therapy. Vol.11 No.4 p. 235 - 248.

Krohne, H. W., de Bruin, J. T., El-Giamal, M. and Schmukle, S. C. (2000) The assessment of surgery-related coping : the coping with surgical stress scale (COSS). Psychology and Health. Vol.15 No.1 p. 135-149.

Krohne, H. W., Hock, M. and Kohlmann, C-W. (1992) Coping dispositions, uncertainty and emotional arousal. In : Strongman, K. T. (ed.) International Review of Studies on Emotion. Volume 2. Chichester : Wiley.

Krohne, H. W., Slangen, K. and Kleemann, P. P. (1996) Coping variables as predictors of peri-operative emotional states and adjustment. Psychology and Health. Vol.11 No.3 p. $315-330$.

Kugler, J., Tenderich, G., Stahlhut, P., Posival, H., Körner, M. M., Körfer, R. and Krüskemper, G. M. (1994a) Emotional adjustment and perceived locus of control in heart transplant patients. Journal of Psychosomatic Research. Vol.38 No.5 p. $403-408$.

Kugler, J., Seelbach, H. Krüskemper, G. M. (1994b) Effects of rehabilitation exercise programmes on anxiety and depression in coronary patients : a metaanalysis. British Journal of Clinical Psychology. Vol.33 No.3 p. $401-410$.

Kulik, J. A., Mahler, H. I. M. and Moore, P. J. (1996) Social comparison and affiliation under threat : effects on recovery from major surgery. Journal of Personality and Social Psychology. Vol.71 No.5 p.967-979.

Lamarche, D., Taddeo, R. and Pepler, C. (1998) The preparation of patients for cardiac surgery. Clinical Nursing Research. Vol.7 No.4 p. $390-405$.

Lancaster, K. A. (1997) Patient teaching in ambulatory surgery. Nursing Clinics of North America. $\quad$ Vol.32 No.2 p. $417-427$. 
Lau, H., Poon, J. and Lee, F. (2000) Patient satisfaction after ambulatory inguinal hernia repair in Hong Kong. Ambulatory Surgery. Vol.8 No.3 p. $115-118$.

Law, M-L. (1997) A telephone survey of day-surgery eye patients. Journal of Advanced Nursing. Vol.25 No.2 p. $355-363$.

Lawrence, K., McWhinnie, D., Jenkinson, C. and Coulter, A. (1997) Quality of life in patients undergoing inguinal hernia repair. Annals of the Royal College $\begin{array}{lll}\text { of Surgeons for England. Vol.79 No.1 } & \text { p. } 40-45 .\end{array}$

Lazarus, R. S. (1966) Psychological Stress and the Coping Process. New York : McGraw-Hill.

Lazarus, R. S. and Folkman, S. (1987) Transactional theory and research on emotions and coping. European Journal of Personality. Vol.1 No.3 p. $141-169$.

Lea, S. G. and Phippen, M. L. (1992) Client education in the ambulatory surgery setting. Seminars in Peri-operative Nursing. Vol.1 No.4 p. 203 223.

Leino-Kilpi, H., lire, L., Suominen, T., Vuorenheimo, J. and Välimäki, M. (1993) Client and information : a literature review. Journal of Clinical Nursing. Vol.2 No.6 p. 331 - 340.

Leino-Kilpi, H. and Vuorenheimo, J. (1993) Peri-operative nursing care quality. Association of Operating room Nurses' Journal. Vol.57 No.5 p. 1061 1071.

Leinonen, T., Leino-Kilpi, H. and Jouko, K. (1996) The quality of intra-operative nursing care : the patient's perspective. Journal of Advanced Nursing. Vol.24 No.4 p. $843-852$.

Leinonen, T., Leino-Kilpi, H., Ståhlberg, M-R. and Lertola, K. (2001) The quality of peri-operative care : development of a tool for the perceptions of patients. Journal of Advanced Nursing. Vol.35 No.2 p. 294 - 306.

Leinonen, T., Leino-Kilpi, H., Ståhlberg, M-R. and Lertola, K. (2001) The quality of peri-operative care : development of a tool for the perceptions of patients. Journal of Advanced Nursing. Vol.35 No.2 p. 294 - 306.

Lepczyk, M., Raleigh, E, H. and Rowley, C. (1990) Timing of pre-operative patient teaching. Journal of Advanced Nursing. Vol.15 No.3 p.300-306.

Lewin, J. M. E. and Razis, P. A. (1995) Prescribing practice of take-home analgesia for day case surgery. British Journal of Nursing. Vol.4 No.18 p. $1047-1051$. 
Ley, P. and Florio, T. (1996) The use of readability formulas in health care. $\begin{array}{llll}\text { Psychology, Health and Medicine. } & \text { Vol.1 No.1 } & \text { p. } 7-28 .\end{array}$

Limb, R. I., Rudkin, G. E., Luck, A. J. Hunt, L. and Hewett, P. J. (2000) The pain of haemorrhoidectomy : a prospective study. Ambulatory Surgery. Vol.8 No.3 p. 129 - 134

Lindeman, C. A. and Van Aernam, B. (1971) The effects of structured and unstructured pre-operative teaching. Nursing Research. Vol.20 No.4 p. $319-332$.

Lindén, I. and Engberg, I. B. (1995) Patients' opinions and experiences of ambulatory surgery : a self-care perspective. Ambulatory Surgery. Vol.3 No.3 p. $131-139$.

Lindén, I. and Engberg, I. B. (1996) Patients' opinions of information given and post-operative problems experienced in conjunction with ambulatory surgery. Ambulatory Surgery. Vol.4 p. $85-91$.

Linden, W., Stossel, C. and Maurice, J. (1996) Psychological interventions for patients with coronary artery disease : a meta-analysis. Archive Intern Medicine. Vol.156 p. $745-752$.

Lisko, S. A. (1995) Development and use of videotaped instruction for preoperative education of the ambulatory gynaecological patient. Journal of Post Anaesthesia Nursing. Vol.10 No.6 p. $324-328$.

Lithner, M., and Zilling, T. (1998) Does pre-operative information increase the well-being of the patient after surgery? Nursing Science and Research in the Nordic Countries. Vol.18 No.1 p. $31-39$.

Litt, M. D., Kalinowski, L. and Shafer. D. (1999) A dental fears typology of oral surgery patients : matching patients to anxiety interventions. Health Psychology. Vol.18 No.6 p. $614-624$.

Litt, M. D., Nye. C. and Shafer, D. (1995) Preparation for oral surgery : evaluating elements of coping. Journal of Behavioural Medicine. Vol.18 No.5 p. $435-459$.

Liu, R., Barry, J. E. S. and Weinman, J. (1994) Effects of background stress and anxiety on post-operative recovery. Anaesthesia. Vol.49 No.5 p. 382 $-386$.

Llewellyn, J. G. (1991) Short stay surgery : present practices, future trends. American Operating Room Nurses' Journal. Vol.53 No.5 p. $1179-1191$. 
Lok C-F. and Bishop, G. D. (1999) Emotion control, stress and health. Psychology and Health. Vol.14 No.5 p. $813-827$.

Lonsdale, M. and Hutchison, G. L. (1991) Patients' desire for information about anaesthesia. Anaesthesia. Vol.46 p. $410-412$.

Ludwick-Rosenthal, R. and Neufeld, R. W. J. (1993) Preparation for undergoing an invasive medical procedure : interacting effects of information and coping style. Journal of Consulting and Clinical Psychology. Vol.61 No.1 p. $156-164$.

MacAndie, C. and Bingham, B. J. G. (1998) Day case nasal surgery : patient satisfaction and the impact on general practitioner workload. Journal of OneDay Surgery. Vol.7 No.3 p. $7-11$.

Mackenzie, J. W. (1989) Daycase anaesthesia and anxiety : A study of anxiety profiles amongst patients attending a day bed unit. Anaesthesia. Vol.44 No.2 p. $437-440$.

Mackintosh, C. and Bowles, S. (1998) Audit of post-operatively pain following day case surgery. British Journal of Nursing. Vol.7 No.11 p. $641-645$.

Mahler, H. I. M. and Kulik, J. A. (1990) Preferences for health care involvement, perceived control and surgical recovery : A prospective study. Social Science and Medicine. Vol.31 No.7 p. $743-751$.

Mahler, H. I. M., Kulik, J. A. and Hill. M. R. (1993) A preliminary report on the effects of videotape preparations for coronary artery bypass surgery on anxiety and self-efficacy: a simulation and evaluation with college students. Basic and Applied Social Psychology. Vol.14 No.4 p. 437 - 453.

Mail on Sunday (2002) The Good Hospital Guide. $10^{\text {th }}$ March.

Male, C. G. (1981) Anxiety in day surgery patients. British Journal of Anaesthesia. Vol.53 No.6 p.663P.

Malin, N. and Teasdale, K. (1991) Caring versus empowerment : considerations for nursing practice. Journal of Advanced Nursing. Vol.16 No.6 p. 657 662.

Mallett, J. and Bailey, C. (1996) The Royal Marsden NHS Trust Manual of Clinical Nursing Procedures. London : Blackwell : Science.

Malster, M. and Parry, A. (2000) Day Surgery In : Manley, K. and Bellman, L. (Eds) Surgical Nursing - Advancing Practice. London : Churchill Livingston. (p. 286). 
Manyande, A., Berg, S., Gettins, D., Stanford, S. C., Mazhero, S., Marks, D. F. and Salmon, P. (1995) Pre-operative rehearsal of active coping imagery influences subjective and hormonal responses to abdominal surgery. Psychosomatic Medicine. Vol.57 No.2 p. $177-182$.

Manyande, A., Chayen, S., Priyakumar, P., Smith, C., Hayes, M., Higgins, D., Kee, S,. Phillips, S. and Salmon, P. (1992) Anxiety and endocrine responses to surgery : Paradoxical effects of pre-operative relaxation training. Psychosomatic Medicine. Vol.54 No.3 p. $275-287$.

Manyande, A and Salmon, P. (1992) Recovery from minor abdominal surgery: A preliminary attempt to separate anxiety and coping. British Journal of Clinical Psychology. Vol.31 No.2 p. $227-237$.

Markanday, L. and Platzer, H. (1994) Brief encounters. Nursing Times. Vol.90 No.7 p. $38-42$.

Markanday, L. (1997) Day Surgery for Nurses. London : Whurr.

Markland, D. and Hardy, L. (1993) Anxiety, relaxation and anaesthesia for day case surgery. British Journal of Clinical Psychology. Vol.32 No.4 p. 493 504.

Marquardt, H. M. and Razis, P. A. (1996) Prepacked take-home analgesia for day case surgery. British Journal of Nursing. Vol.5 No.18 p. 1114-1118.

Marteau, T. M. and Bekker, H. (1992) The development of a six-item short form of the state scale of the Spielberger state-trait anxiety inventory. British Journal of Clinical Psychology. Vol.31 Pt.3 p. $301-306$.

Martelli, M. F., Auerbach, S. M., Alexander, J. and Mercuri, L. G. (1987) Stress management in the health care setting: Matching interventions with patient coping styles. Journal of Consulting and Clinical Psychology. Vol.55 No.2 p. $201-207$.

Martikainen, M., Kangas-Saarela, T., Löppönen, A. and Salomäki, T. (2000) One-week recovery profiles after spinal, propofol, isoflurane and desflurane anaesthesia in ambulatory knee arthroscopy. Ambulatory Surgery. Vol.8 No.3 p. $139-142$.

Mathews, A and Ridgeway, V. (1984) Psychological preparation for surgery. In : Steptoe, A. and Mathews, A. (eds.) Health Care and Human Behaviour. London : Harcourt Brace Jovanovch. p. 231.

McCleane, G. J. and Cooper, R. (1990) The nature of pre-operative anxiety. Anaesthesia. Vol.45 No.2 p. $153-155$. 
McCallum, J., Nakamura, T., Bye, R. and Jackson, D. (2000) Day surgery for older people $(70+)$ : selection versus outcome effects. Ambulatory Surgery. Vol.8 No.3 p. $143-149$.

McGaw, C. D. and Hanna, W. J. (1998) Knowledge and fears of anaesthesia and surgery : the Jamaican perspective. West Indian Medical Journal. Vol.47 No.2 p. $64-67$.

Mealy, K., Ngeh, N., Gillen, P., Fitzpatrick, G., Keane, F. B. V. and Tanner, A. (1996) Propranolol reduces the anxiety associated with day case surgery. European Journal of Surgery. Vol.162 No.1 p. $11-14$.

Mechanic, D. (1980) The experience and reporting of common physical complaints. Journal of Health and Social Behaviour. Vol.21 No.2 p. 146 155.

Meeker, M. H. and Rothrock, J. C. (1999) (11 ${ }^{\text {th }}$ Ed) Alexander's Care of the Patient in Surgery. London : Mosby.

Menon, N. K. (1998) Patient satisfaction with direct access vasectomy service. British Journal of Family Planning. Vol.24 No.3 p. $105-106$.

Meredith, P. (1993) Patient satisfaction with communication in general surgery : Problems of measurement and improvement. Social Science and Medicine. Vol.37 No.5 p. $591-602$.

Michaels, J. A., Reece-Smith, H. and Faber, R. G. (1992) Case-control study of patient satisfaction with day-case and in-patient inguinal hernia repair. Journal of the Royal College of Surgeons of Edinburgh. Vol.37 No. 2 p. $99-100$.

Milgram, S. (1974) Obedience to Authority. London : Tavistock.

Miller, S. M. (1980) When is a little information a dangerous thing? Coping with stressful events by monitoring versus blunting. In : Levine, S. and Ursin, $\mathrm{H}$. (eds.) Coping and Health. London : Plenum Press. p. 145.

Miller, G. E. and Cohen, S. (2001) Psychological interventions and the immune system : A meta-analytic review and critique. Health Psychology. Vol.20 No.1 p. $47-63$.

Miller, S. M., Brody, D. S. and Summerton, J. (1988) Styles of coping with threat : implications for health. Journal of Personality and Social Psychology. Vol.54 No.1 p. 142 - 148.

Miller, S. M., Combs, C. and Stoddard, E. (1989) Information, coping and control in patients undergoing surgery and stressful medical procedures. In : Steptoe, A. and Appels, A. (eds.) Personal Control and Health. Chichester : Wiley. p. 107. 
Miller, S. M. and Mangan, C. E. (1983) Interacting effects of information and coping style in adapting to Gynaecologic stress : Should the doctor tell all? Journal of Personality and Social Psychology. Vol.45 No.1 p. 223 - 236.

Miller, S. M., Mischel, W., Schroeder, C. M., Buzaglo, J. S., Hurley, K., Schreiber. P. and Mangan, C. (1998) Intrusive and avoidant ideation among females pursuing infertility treatment. Psychology and Health. Vol.13 No.5 p. $847-858$.

Miller, S. M., Roussi, P., Caputo, G. C. and Kruus, L. (1995) Patterns of children's coping with an aversive dental treatment. Health Psychology. Vol.14 No.3 p. $236-246$.

Miller, S. M., Rodoletz, M., Schroeder, C. M., Mangan, C. E. and Sedlacek, T. V. (1996) Applications of the monitoring process model to coping with severe long-term medical threats. Health Psychology. Vol.15 No.3 p. 216 225.

Mills, R. T. and Krantz, D. S. (1979) Information, choice and reactions to stress : a field experiment in a blood bank with laboratory analogue. Journal of Personality and Social Psychology. Vol.37 No.4 p. $608-620$.

Miró, J., and Raich, R. M. (1999) Effects of a brief and economical intervention in preparing patients for surgery : does coping style matter? Pain. Vol.83 No.3 p. $471-475$.

Mitchell, M. J. (1994) Pre-operative and post-operative psychological nursing care. Surgical Nurse. Vol.7 No.3 p. 22 - 25.

Mitchell, M. J. (1997) Patients` perceptions of pre-operative preparation for day surgery. Journal of Advanced Nursing. Vol.26 No.2 p. $356-363$.

Mitchell, R. B., Kenyon, G. S. and Monks, P. S. (1999) A cost analysis of daystay surgery in otolaryngology. Annals of the Royal College of Surgeons of England. (Supplement). Vol.82 March p. 85-92.

Moerman, N., van Dam, F. S. A. M., Muller, M. J. and Oosting, H. (1996) The Amsterdam pre-operative anxiety and information scale. Anesthesia Analgesia. Vol.82 No.3 p. $445-451$.

Mogg, K., Mathews, A., Bird, C. and MacGregor-Morris, R. (1990) Effects of stress and anxiety on the processing of threat stimuli. Journal of Personality and Social Psychology. Vol.59 No.6 p. $1230-1237$.

Montori, A. (1998) Minimally invasive surgery. Endoscopy. Vol.30 No.2 p. $244-252$. 
Moon, J-S. and Cho, K-S. (2001) The effects of handholding on anxiety in cataract surgery patients under local anaesthesia. Journal of Advanced Nursing. Vol.35 No.3 p. $407-415$.

Mott, A. M. (1999) Psychologic preparation to decrease anxiety associated with $\begin{array}{llll}\text { cardiac catheterisation. Journal of Vascular Nursing. Vol.17 No.2 } & \text { p. } 41\end{array}$ $-49$.

Motyka, M., Motyka, H. and Wsolek, R. (1997) Elements of psychological support in nursing care. Journal of Advanced Nursing. Vol.26 No.5 p. $909-912$.

Mudie, S. (1992) Evaluating day care. Nursing (UK). Vol.5 No.7 p. 22 - 23.

Mukumba, S., Wright, M. and Punchihewa, V. G. (1996) Notes on an unlimited telephone helpline. Journal of One Day Surgery. Vol.6 No.3 p. 14.

Mullen, B. and Suls, J. (1982) The effectiveness of attention and rejection as coping styles : a meta-analysis of temporal differences. Journal of Psychosomatic Research. Vol.26 No.1 p. $43-49$.

Mumford, E., Schlesinger, H. J. and Glass, G. V. (1982) The effects of psychological intervention on recovery from surgery and heart attacks : an analysis of the literature. American Journal of Public Health. Vol.72 No.2 p. $141-151$.

Murphy, S. J. (1994) Pre-operative assessment for day surgery. Surgical Nurse. Vol.7 No.3 p. $6-9$.

Nettina, S. N. (1996) $\left(6^{\text {th }}\right.$ Ed) Lippincott Manual of Nursing Practice. Philadelphia : Lippincott.

N.H.S. Management Executive Value for Money Unit (1991) Day Surgery : Making it Happen. London: HMSO.

N.H.S. Management Executive (1993) Report by the Day Surgery Task Force. London : HMSO. (Updated in 1994).

Nkyekyer, K. (1996) Day-case laparoscopy in a Ghanaian teaching hospital : the patients' perspective. Tropical Doctor. Vol.26 No.4 p. 147-150.

Nyamathi, A. and Kashiwabara, A. (1988) Pre-operative anxiety. Association of Operating Room Nurses' Journal. Vol.47 No.1 p. $164-169$.

Oberle, K. (1991) A decade of research in locus of control : what have we learned? Journal of Advanced Nursing. Vol.16 No.7 p. $800-806$. 
Oberle, K., Allen, M. and Lynkowski, P (1994) Follow up of same day surgery patients : a study of patient concerns. Association of Operating Room Nurses' Journal. Vol.59 No.5 p. $1016-1018,1021-1025$.

O Connor, S. J., Gibberd, R. W. and West, P. (1991) Patient satisfaction with day surgery. Australian Clinical Review. Vol.11 No.4 p. 143-149.

O'Hara, M. W., Ghoneim, M. M., Hinrichs, J. V., Mehta, M. P., and Wright, E. J. (1989) Psychological consequences of surgery. Psychosomatic Medicine. Vol.51 No.3 p. 356 - 370 .

Ong, E. L., Chiu, J. W., Chong, J. L. and Kwan, K. M. (2000) Volatile induction and maintenance (VIMA) versus total intravenous anaesthesia (TIVA) for minor gynaecological procedures. Ambulatory Surgery. Vol.8 No.1 p. $37-40$.

O'Rourke, A., Lewin, B., Whitecross, S. and Pacey, W. (1991) The effects of physical exercise training and cardiac education on levels of anxiety and depression in the rehabilitation of coronary artery bypass graft patients. International Disability Studies. Vol.12 No.3 p. $104-106$.

Otte, D. I. (1996) Patients' perspectives and experiences of day surgery. Journal of Advanced Nursing. Vol.23 No.6 p. $1228-1237$.

Padilla, G. V., Grant, M. M., Rains, B. L., Hansen, B. C. Bergrstrom, N., Wong, H. L., Hansen, R. and Kubo, W. (1981) Distress reduction and the effects of preparatory teaching films and patient control. Research in Nursing and Health. Vol.4 No.4 p. $375-387$.

Parlow, J. L., Meikle, A. T., van Vlymen, J. and Avery, N. (1999) Post-operative nausea and vomiting after ambulatory laparoscopy is not reduced by $\begin{array}{lll}\text { promethazine prophylaxis. Canadian Journal of Anesthesia. Vol.46 No.8 } & \text { No }\end{array}$ p. $719-724$.

Parsons, E. C., Kee, C. C. and Gray, P. (1993) Peri-operative nursing caring behaviours. Association of Operating Room Nurses' Journal. Vol.57 No.5 p. $1106-1114$.

Peerbhoy, D., Hall, G. M., Parker, C., Shenkin, A. Salmon, P. (1998) Patients' reactions to attempts to increase passive or active coping with surgery. Social Science and Medicine. Vol.47 No.5 p.595-601.

Penn, S., Davenport, H. T., Carrington, S. and Edmondson, M. (1996) Principles of Day Surgery. London : Blackwell Science.

Perez, E. M., Barriga, R., Rodriguez, M. A., Larranaga, E., Figueroa, J. M. and Serrano, P. A. (2000) Ambulatory surgery for groin hernia : the Gilbert repair. Ambulatory Surgery. Vol.8 No.3 p. $135-138$. 
Peterson, C and Stunkard, A. J. (1989) Personal control and health promotion. Social Science and Medicine. Vol.28 No.8 p. $819-828$.

Petticrew, M., Black, N. A. and Moore, L. (1995) Day surgery dilatation and curettage : patients' experiences. Ambulatory Surgery. Vol.3 No.4 p. 185 $-188$.

Pfisterer, M., Ernst, E. M., Hirlekar, G., Maser, P., Shaalan, A. K., Haigh, C. and Upadhyaya, B. (2001) Post-operative nausea and vomiting in patients undergoing day-case surgery : an international, observational study. Ambulatory Surgery. Vol.9 No.1 p. $13-18$.

Philip, B. K. (1992) Patients' assessment of ambulatory anaesthesia and surgery. Journal of Clinical Anaesthesiology. Vol.4 No.5 p. 355 - 358.

Pickett, C. and Clum, G. A. (1982) Comparative treatment strategies and their interaction with locus of control in the reduction of post-surgical pain and anxiety. Journal of Consulting and Clinical Psychology. Vol.50 No.3 p. $439-441$.

Pineault, R., Contandriopoulos, A-P., Valois, M., Bastian, M-L. and Lance, J-M. (1985) Randomised clinical trial of one-day surgery : patient satisfaction, clinical outcomes and costs. Medical Care. Vol.23 No.2 p. 171-182.

Pollock, I. and Trendholm, J. (1997) All in a Day's Work. Which? : Independent Consumer Guide. February. p. $14-17$.

Räikkönen, K., Mathews, K. A., Flory, J. D., Owens, J. F. Gump, B. B. (1999) Effects of optimism, pessimism, and trait anxiety on ambulatory blood pressure and mood during everyday life. Journal of Personality and Social Psychology. Vol.76 No.1 p. $104-113$.

Ramachandra, V. (1994) Day surgery pain. Journal of One-Day Surgery. Vol.3 No.4 p. $14-15$.

Ramsay, M. A. E. (1972) A survey of pre-operative fear. Anaesthesia. Vol.27 No.4 p. $396-402$.

Ratcliffe, F., Lawson, R. and Millar, J. (1994) Day-case laparoscopy revisited : have post-operative morbidity and patient acceptance improved? Health Trends. Vol.26 No.2 p. $47-49$.

Ray, C. and Fitzgibbon, G. (1981) Stress arousal and coping with surgery. Psychological Medicine. $\quad$ Vol.11 No.4 p. $741-746$.

Read, D. (1990) Day surgery : A consumer survey. New Zealand Medical Journal. Vol.103 No.895 p. $369-371$. 
Reading, A. E. (1982) Effects of psychological preparation on pain and recovery after minor gynaecological surgery : A preliminary report. Journal of Clinical Psychology. Vol.38 No.3 p.504-514.

Redman, B. K. (1993) Patient education at 25 years : where we have been and where we are going. Journal of Advanced Nursing. Vol.18 No.5 p. 725 730 .

Reid, J. H. (1997) Meeting the informational needs of patients in a day surgery setting : An exploratory level study. British Journal of Theatre Nursing. Vol.7 No.4 p. $19-24$.

Richert, A. J. (1981) Sex differences in relation of locus of control and reported anxiety. Psychological Reports. Vol.49 No.3 p. $971-974$.

Ridgeway, V. and Mathews, A. (1982) Psychological preparation for surgery : A comparison of methods. British Journal of Clinical Psychology. Vol.21 No.4 p. $271-280$.

Rogers, M. and Reich, P. (1986) Psychological intervention with surgical patients. Advanced Psychosomatic Medicine. Vol.15 p. 23 - 50.

Rosenbaum, M. and Piamenta, R. (1998) Preference for local or general anesthesia, coping dispositions, learned resourcefulness and coping with surgery. Psychology and Health. Vol.13 No.5 p. 823-845.

Roth, S and Cohen, L. J. (1986) Approach, avoidance, and coping with stress. American Psychologist. Vol.41 No.7 p. 813-819.

Rothrock, J. C. (1989) Peri-operative nursing research. Part I : Pre-operative psycho-educational interventions. Association of Operating Room Nurses' Journal. Vol.49 No.2 p. $597-619$.

Rotter, J. (1966) Generalised expectancies for internal vs. external control of reinforcement. Psychological Monographs. Vol.80 No.1 p.1-26.

Royal College of Surgeons of England (1992) Guidelines for day case surgery London : HMSO.

Royal College of Surgeons of England and East Anglia Regional Health Authority (1995) New Angles on Day Surgery. NHS Executive : East Anglian Regional Clinical Audit Office.

Royal College of Surgeons of England and Royal College of Psychiatrists (1997) Report on the Working Party on the Psychological Care of Surgical Patients (CR55) London : RCS \& RCP. 
Rudkin, , G. E., Bacon, A. K., Burrow, B., Chapman, M. H., Claxton, M., Donovan, B., Gibb, D. and Weber, L. S. (1996) Review of efficiencies and patient satisfaction in Australian and New Zealand day surgery units : a pilot study. Anaesthesia and Intensive Care. Vol.24 No.1 p. 74 - 78.

Ruuth-Setälä, A., Leino-Kilpi, H. and Suominen, T. (2000) How do I manage at home? Where do Finnish short-stay patients turn for help, support and company after discharge, and why? Journal of One-Day Surgery. Vol.10 No.1 p. $15-18$.

Ryan, D. W. (1975) A questionnaire survey of pre-operative fears. British Journal of Clinical Practice. Vol.29 No.1 p. 3 - 6.

Salmon, P. (1992a) Surgery as a psychological stressor: Paradoxical effects of pre-operative emotional state on endocrine response. Stress Medicine. Vol.8 No.3 p. 193 - 198.

Salmon, P. (1992b) Psychological factors in surgical stress : Implications for management. Clinical Psychology Review. Vol.12 No.7 p. $681-704$.

Salmon, P. (1993) The reduction of anxiety in surgical patients: An important nursing task of medicalisation of preparatory worry. International Journal of Nursing Studies. Vol.30 No.4 p. $323-330$.

Salmon, P., Evans, R. and Humphrey, D. E. (1986) Anxiety and endocrine changes in surgical patients. British Journal of Clinical Psychology. Vol.25 No.2 p. $135-141$.

Salmon, P and Hall, G. M. (1997) A theory of post-operative fatigue. Journal of the Royal Society of Medicine. Vol.90 No.12 p.661-664.

Salmon, P., Shah, R., Berg, S. and Williams, C. (1994) Evaluating customer satisfaction with colonoscopy. Endoscopy. Vol.26 No.4 p. $342-346$.

Salvage, J. (1990) The theory and practice of the 'New nursing'. Nursing Times. Vol.86 No.1 p. $42-454$.

Sanderson. I. M. (1998) The Clinical Negligence Scheme for Trusts : A review of its present function. Clinical Risk. Vol.4 p. 35 - 43.

Scheier, M. F., and Carver, C. S. (1992) Effects of optimism on psychological and physical well-being : theoretical overview and empirical update. Cognitive Therapy and Research. Vol.16 No.2 p. $201-228$.

Schoessler, M (1989) Perceptions of pre-operative education in patients admitted the morning of surgery. Patient Education and Counselling. Vol.14 No.2 p. $127-136$. 
Schröder, K. E. E. and Schwarzer, R. (1998) Coping as a mediator from recovery from cardiac surgery. Psychology and Health. Vol.13 No.1 p. $83-97$.

Schwartz-Barcott, D., Fortin, J. D. and Kim, H. S. (1994) Client/ Nurse interaction : Testing for its impact on pre-operative instruction. International Journal of Nursing Studies. Vol.31 No.1 p. $23-35$.

Schweizer, K., Beck-Seyffer, A. and Schneider, R. (1999) Cognitive bias of optimism and its influence on well-being. Psychological Reports. Vol.84 No.2 p. $627-636$.

Scioli, A., Chamberlin, C. M., Samor, C. M., Lapointe, A. B., Campbell, T. L. and Macleod, A. R. (1997) A prospective study of hope, optimism and health. $\begin{array}{lllll}\text { Psychological Reports. } & \text { Vol.81 No.3 Part1 } & \text { p. } 723-733 .\end{array}$

Scriven, A. and Tucker, C. (1997) The quality and management of written information presented to women undergoing hysterectomy. Journal of Clinical Nursing. Vol.6 No.2 p. $107-113$.

Seeman, M. and Seeman, T. E. (1983) Health behaviour and personal autonomy : A longitudinal study of the sense of control in illness. Journal of Health and Social Behaviour. Vol.24 No.6 p. $144-160$.

Segerstrom, S. C., Taylor, S. E. Kemeny, M. E. and Fahey, J. L. (1998) Optimism is associated with mood, coping and immune change in response to stress. Journal of Personality and Social Psychology. Vol.74 No.6 p. $1646-1655$.

Sherman, A., Higgs, G. E. and Williams, R. L. (1997) Gender differences in the locus of control construct. Psychology and Health. Vol.12 No.2 p. 239 248.

Shipley, R. H., Butt, J. H., Horwitz, B. and Farbry, J. E. (1978) Preparation for a stressful medical procedure : Effect of amount of stimulus pre-exposure and coping style. Journal of Consulting and Clinical Psychology. Vol.46 No.3 p. $499-507$.

Shipley, R. H., Butt, J. H. and Horwitz, B. (1979) Preparation to re-experience a stressful medical examination : effect of repetitious videotape exposure and $\begin{array}{llll}\text { coping style. Journal of Consulting and Clinical Psychology. } & \text { Vol.47 No.3 }\end{array}$ p. $485-492$.

Shuldham, C. (1999) A review of the impact of pre-operative education on recovery from surgery. International Journal of Nursing Studies. Vol.36 No.2 p. $171-177$. 
Sigurdardottir, A. K. (1996) Satisfaction among ambulatory surgery patients in $\begin{array}{llll}\text { two hospitals in Iceland. Journal of Nursing Management. Vol.4 No.2 } & \text { No }\end{array}$ p. $69-74$.

Sime, A. M. (1976) Relationship of pre-operative fear, type of coping, and information received about surgery to recovery from surgery. Journal of Personality and Social Psychology. Vol.34 No.4 p. 716-724.

Singleton, R. J., Rudkin, G. E., Osborne, G. A., Watkin. D. S. and Williams, J. A. R. (1996) Laparoscopic cholecystectomy as a day surgery procedure. Anaesthesia and Intensive Care. Vol.24 No.2 p. 231-136.

Smith, R. and Draper, P. (1994) Who is in control? An investigation of nurse and patient beliefs relating to control of their health care. Journal of Advanced Nursing. Vol.19 No.5 p. $884-892$.

Smith, J. (1998) Patient satisfaction survey : Inguinal hernia repair performed in a primary setting. Journal of One-Day Surgery. Vol.8 No.1 p. 10-16.

Solcova, I. and Sykora, J. (1995) Relationship between psychological hardiness and physiological response. Homeostasis. Vol.36 No.1 p. $30-34$.

Spector, P. E. and Sistrunk, F. (1979) Reassurance : A mechanism by which the presence of others reduces anxiety. Journal of Social Psychology. Vol.109 p. $119-126$.

Spielberger, C. D., Gorsuch, R. L., Lushene, R., Vagg, P. R. and Jacobs, G. A. (1983) Manual for the State-Trait Anxiety Inventory for Adults. Palo Alto California : Consulting Psychologists Press.

Spitzer, A. (1998) Moving into the information era : does the current nursing paradigm still hold? Journal of Advanced Nursing. Vol.28 No.4 p. 786 793.

Stanley, B. M., Walters, D. J. and Maddern, G. J. (1998) Informed consent : how much information is enough? Australian and New Zealand Journal of Surgery. Vol.68 No.11 p.788-791.

Stengrevics, S., Sirois, C., Schwartz, C. E., Friedman, R. and Domar, A. D. (1996) The prediction of cardiac surgery outcome based upon pre-operative psychological factors. Psychology and Health. Vol.11 No.4 p. 471-477.

Stephenson, M. (1988) The case for day care surgery. Nursing Times. Vol.84 No.4 p. $37-38$.

Stephenson, M. E. (1990) Discharge criteria in day surgery. Journal of Advanced Nursing. Vol.15 No.5 p.601-613. 
Stevens, R. (1989) Freud and Psychoanalysis. Milton Keynes : Open University Press.

Strickland, B. R. (1978) Internal-External expectancies and health related behaviours. Journal of Consulting and Clinical Psychology. Vol.46 No.6 p. $1192-1211$.

Strull, W. M., Lo, B. and Charles, G. (1984) Do patients want to participate in medical decision making? Journal of the American Medical Association. Vol.252 No.21 p. $2990-2994$.

Suls, J., David, J. P., and Harvey, J. H. (1996) Personality and coping : three generations of research. Journal of Personality. Vol.64 No.4 p. 711 735.

Suls, J. and Fletcher, B. (1985) The relative efficacy of avoidant and nonavoidant coping strategies : a meta analysis. Health Psychology. Vol.4 No.3 p. $249-288$.

Suls, J. and Wan, C. K. (1989) Effects of sensory and procedural information on coping with stressful medical procedures and pain : A meta analysis. Journal of Consulting and Clinical Psychology. Vol.57 No.3 p. 372 - 379.

Sunday Times (2001) Good Hospital Guide. $14^{\text {th }}$ and $21^{\text {st }}$ January.

Swindale, J. E. (1989) The nurse's role in giving pre-operative information to reduce anxiety in patients admitted to hospital for elective minor surgery. Journal of Advanced Nursing. Vol.14 No.11 p. 899 - 905.

Taylor, S. E. and Lobel, M. (1989) Social comparison activity under threat : downward evaluation and upward contacts. Psychological Review. Vol.96 No.4 p. $569-575$.

Teasdale, K. (1989) The concept of reassurance in nursing. Journal of Advanced Nursing. Vol.14 No.6 p. $444-450$.

Teasdale, K. (1993) Information and anxiety : a critical reappraisal. Journal of Advanced Nursing. Vol.18 No.7 p. $1125-1132$.

Teasdale, K. (1995a) The nurse's role in anxiety management. Professional $\begin{array}{llll}\text { Nurse. } & \text { Vol.10 No.8 } & \text { p. } 509-512 .\end{array}$

Teasdale, K. (1995b) Theoretical and practical considerations on the use of reassurance in the nursing management of anxious patients. Journal of Advanced Nursing. Vol.22 No.1 p. $79-86$.

Thatcher, J. (1996) Follow-up after day surgery : how well do patients cope? $\begin{array}{llll}\text { Nursing Times. Vol.92 No.37 } & \text { p. } 30-32 .\end{array}$ 
Thomas, H. and Hare. M. J. (1987) Day case laparoscopic sterilisation - time for $\begin{array}{llll}\text { a rethink. British Journal of Obstetrics and Gynaecology. Vol.94 No.5 } & \text { No }\end{array}$ p. $445-448$.

Thomas, J. J. (1995) Reducing anxiety during phase I cardiac rehabilitation. Journal of Psychosomatic Research. Vol.39 No.3 p. 295 - 304.

Tix, A. P. and Frazier, P. A. (1998) The use of religious coping during stressful life events : main effects, moderation and mediation. Journal of Consulting and Clinical Psychology. Vol.66 No.2 p. $411-422$.

Tongue, B. and Stanley, I. (1991) A video-based information system for patients. Health Trends. Vol.23 No.1 p. 11-12.

Towey, R. M. Stanford, B. J. Ballard, R. M. and Gilbert, J. R. (1979) Morbidity of day-case gynaecological surgery. British Journal of Anaesthesia. Vol.51 No.5 p. $453-455$.

Van Balen, F, and Verdurmen, J. (1999) Medical anxiety and the choice for treatment : the development of an instrument to measure fear of treatment. Psychology and Health. Vol.14 No.5 p. 927 - 935.

Van Zuuren, F. J., De Groot, K. I., Mulder, N. L. and Muris, P. (1996) Coping with medical threat : an evaluation of the threatening medical situations inventory (TMSI) Personality and Individual Differences. Vol.21 No.1 p. 21 31.

Van Zuuren, F. J., De Jongh, A., Beekers, C. and Swinkels, P. (1999) Coping with dental treatment : correlates of dispositional and domain specific monitoring and blunting. Psychology and Health. Vol.14 No.2 p.323-337.

Vogele, C. and Steptoe, A. (1986) Physiological and subjective stress responses in surgical patients. Journal of Psychosomatic Research. Vol.30 No.2 p. $205-215$.

Vogelsang, J. (1990) Continued contact with a familiar nurse affects women's perceptions of the ambulatory surgical experience : a qualitative-quantitative design. Journal of Post Anaesthetic Nursing. Vol.5 No.5 p. 315-320.

Volicer, B. J. (1973) Perceived stress levels of events associated with the experience of hospitalisation : Development and testing of a measurement tool. Nursing Research. Vol.22 No.6 p. $491-497$.

Volicer, B. J. and Bohannon, W. M. (1975) A hospital stress rating scale. Nursing Research. Vol.24 No.5 p. $352-359$. 
Waisel, D. B. and Truog, R. D. (1995) The benefits of the explanation of risks of anaesthesia in the day surgery patient. Journal of Clinical Anaesthesia. Vol.7 No.3 p. $200-204$.

Wallace, L. M. (1986a) Communication variables in the design of pre-surgical preparatory information. British Journal of Clinical Psychology. Vol.25 No.2 p. $111-118$.

Wallace, L. M. (1986b) Pre-operative state anxiety as a mediator of psychological adjustment to and recovery from surgery. British Journal of Medical Psychology. Vol.59 No.3 p. $253-261$.

Wallace, L. M. (1986c) Day-case laparoscopy : patient preferences, adjustment and management. Journal of Psychosomatic Obstetrics and Gynaecology. Vol.5 No.3 p. $207-216$.

Wallace, L. M. (1987) Trait anxiety as a predictor of adjustment to and recovery $\begin{array}{lllll}\text { from surgery. British Journal of Clinical Psychology. Vol.26 No.1 p. } 73 & \end{array}$ $-74$.

Wallston, K. A. (1992) Hocus-Pocus, the focus isn't strictly on locus : Rotter's social learning theory modified for health. Cognitive Therapy and Research. Vol.16 No.2 p.183- 199.

Wallston, K. A., Malcarne, V. L., Flores, L., Hansdottir, I., Smith, C. A., Stein, M. J., Weisman, M. H. and Clements, P. J. (1999) Does god determine your health? The god locus of health control scale. Cognitive Therapy and Research. Vol.23 No.2 p. $131-142$.

Wallston, K. A., Wallston, B. S. and DeVellis, R. (1978) Development of the multidimensional health locus of control (MHLC) scales. Health Education Monographs. Vol.6 No.2 p.160-170.

Wallston, K. A. and Wallston, B. S. (1982) Who is responsible for your health? The construct of health locus of control. In : Sanders, G. and Suls, J. (eds.) Social Psychology of Health and Illness. London : Lawrence Erlbaum. p. 65.

Walsh, M. and Ford, P. (1989) Nursing Rituals : Research and Rational Actions. London : Heinemann. Ch. 1.

Walsh, D. and Shaw, D. G. (2000) The design of written information for cardiac patients : a review of the literature. Journal of Clinical Nursing. Vol.9 No.5 p. $658-667$.

Webb, C. and Hope, K. (1995) What kind of nurses do patients want? Journal of Clinical Nursing. Vol.4 No.2 p. $101-108$. 
Webber, G. C. (1990) Patient Education : A Review of the Issues. Medical Care. Vol.28 Part 2 No.11 p. $1089-1103$.

Wedderburn, A. W., Morris, G. E. and Dodds, S. R. (1996) A survey of postoperative care after day case surgery. Annals of the Royal College of Surgeons $\begin{array}{lll}\text { of England. Vol.78 No.2 } & \text { p.70 }-71 .\end{array}$

Wells, J. K., Howard, G. S., Nowlin, W. F. and Vargas, M. J. (1986) Pre-surgical anxiety and post-surgical pain and adjustment: Effects of a stress inoculation $\begin{array}{llll}\text { procedure. Journal of Consulting and Clinical Psychology. Vol.54 No.6 } & \text { No. }\end{array}$ p. $831-835$.

Whitwam, J. G. (1994) Day-Case Anaesthesia and Sedation. London : Blackwell Scientific Publications.

Wicklin, N. and Forster, J. (1994) The effects of a personal versus a factual approach video-tape on the level of pre-operative anxiety of same day surgery patients. Patient Education and Counselling. Vol.23 No.2 p. 107-114.

Wilkinson, D., Bristow, A. and Higgins, D. (1992) Morbidity following day surgery. Journal of One-Day Surgery. Vol.2 No.1 p.5-6.

Willis, C. E. Watson, J. D. Harper, C. V. and Humphreys, W. G. (1997) Does day surgery embarrass the primary health care team? : An audit of complications and consultations. Ambulatory Surgery. Vol.5 No.2 p.71 $-75$.

Willsher, P. C., Urbach, G., Cole, D., Schumacher, S. and Litwin, D. E. M. (1998) Outpatient laparoscopic surgery. Australia and New Zealand Journal of Surgery. Vol.68 No.11 p. $769-773$.

Wilson, J. F. (1981) Behavioural preparation for surgery : benefit or harm? Journal of Behavioural medicine. Vol.4 No.1 p. $79-102$.

Wilson-Barnett, J. (1976) Patient's emotional reactions to hospitalisation: An exploratory study. Journal of Advanced Nursing. Vol.1 No.5 p. 351 - 358.

Wilson-Barnett, J. (1984) Interventions to alleviate patients' stress : A review. Journal of Psychosomatic Research. Vol.28 No.1 p. 63 - 72.

Wilson-Barnett, J. and Osborne, J. (1983) Studies evaluating patient teaching: implications for practice. International Journal of Nursing Studies. Vol.20 No.1 p. $33-44$.

Winwood, M. A. and Jago, R. H. (1993) Anxiety levels following anaesthesia for day case surgery. Anaesthesia. Vol.48 No.7 p. $581-584$. 
Woodhouse, M., King, T. A. and Challiner, A. (1998) Impact of day surgery on community services. Journal of One-Day Surgery. Vol.8 No.1 p. 3-4.

Young, L. and Humphrey, M. (1985) Cognitive methods of preparing women for Hysterectomy : does a booklet help? British Journal of Clinical Psychology. Vol.24 No.4 p. $303-304$.

Young, R., de Guzman, C. P., Mantis, M. S. and McClure, K. (1994) Effect of pre-admission brochures on surgical patients' behavioural outcomes. Association of Operating Room Nurses` Journal. Vol.60 No.2 p. 232 241.

Yount, S. and Schoessler, M. (1991) A description of patient and nurse perceptions of pre-operative teaching. Journal of Post Anaesthesia Nursing. Vol.6 No.1 p. $17-25$.

Ziemer, M. M. (1983) Effects of information on post-surgical coping. Nursing Research. Vol.32 No.5 p. $282-287$.

Zvara, D. A., Mathes, D. D., Brooker, R. F. and McKinley, A. C. (1996) Video as a patient teaching tool : Does it add to the pre-operative anesthetic visit? Anesthesia Analgesia. Vol.82 No.5 p. $1065-1068$. 


\section{Bibliography}

Bell, J. (1993) $\left(2^{\text {nd }}\right.$ Ed.) Doing Your Research Project. Buckingham : Open University Press.

Cahill, H. and Jackson, I. (1997) Day Surgery : Principles and Nursing Practice. London : Baillière Tindall.

Conner, M. and Norman, P. (1996) Predicting Health Behaviour. Buckingham : Open University Press.

Coolican, H (2000) ( $3^{\text {rd }}$ Ed) Research methods and statistics in psychology. London : Hodder and Stoughton.

Cooper, C. L. and Payne, R. (1991) Personality and Stress : Individual Differences in the Stress Process. Chichester : Wiley and Sons.

Corston, R. and Colman, A. (2000) A Crash Course in SPSS for Windows. Oxford : Blackwell Publishers.

Couchman, W. and Dawson, J. (1990) Nursing and Health Care Research : A Practical Guide. London : Scutari Press.

Foster, J. J. (1993) (2 $2^{\text {nd }}$ Ed.) Starting SPSS/ PC+ and SPSS for Windows. Wilmslow : Sigma.

Field, A (2000) Discovering statistics using SPSS for windows. London : Sage.

Gross, R. D. (1992) (2 ${ }^{\text {nd }}$ Ed.) Psychology : The Science of Mind and Behaviour. London : Hodder and Stoughton.

Gross, R., Humphreys, P. and Petkova, B. (1997) Challenges in Psychology. London : Hodder and Stoughton.

Howell, D. C. (1995) (3 ${ }^{\text {rd }}$ Ed) Fundamental Statistics for the Behavioural Sciences. California : Duxbury Press.

Kinnear, P. R. and Gray, C. D. (1997) $\left(2^{\text {nd }}\right.$ Ed) SPSS for Windows Made Simple. United Kingdom : Psychology Press.

Mason, C. and McKenna, H. (1995) How to survive a PhD. Nurse Researcher. Vol.2 No.3 p. $73-79$.

Monat, A. and Lazarus, R. S. (1991) ( $3^{\text {rd }}$ Ed.) Stress and Coping : An Anthology. New York : Columbia University Press.

Neale, J. M. and Liebert, R. M. (1986) ( ${ }^{\text {rd }}$ Ed.) Science and Behaviour : An Introduction to Methods of Research. London: Prentice-Hall. 
Oppenheim, A. N. (1992) Questionnaire Design, Interviewing and Attitude Measurement. London : Pinter Publications.

Parahoo, K. (1997) Nursing Research : Principles, Process and Issues. London : Macmillan.

Penn, S., Davenport, H. T., Carrington, S. and Edmondson, M. (1996) Principles of Day Surgery. London : Blackwell Science.

Phillips, E. M. and Pugh, D. S. (1994) ( $2^{\text {nd }}$ Ed.) How to Get a PhD. A Handbook for Students and their Supervisors. Buckingham : Open University Press.

Pitts, M. and Philips, K. (1991) The Psychology of Health : An Introduction. London : Routledge.

Polite, D. F. and Hungler, B. P. (1991) $\left(4^{\text {th }}\right.$ Ed.) Nursing Research : Principles and Methods. London : Lippincott.

Sirkin, R. M. (1999) (2 $2^{\text {nd }}$ Ed.) Statistics for the Social Sciences. London : Sage Publications.

Whitwam, J. G. (1994) Day-Case Anaesthesia and Sedation. London : Blackwell Scientific Publications. 